\title{
Going National with HERS and EEMs: Issues and Impacts
}

DE92 001220

\section{The Collected Paperss of the National Collaborative}

National Collaborative on Home Energy Rating Systems and Mortgage Incentives for Energy Efficiency

Prepared under Task No. AS135440 by

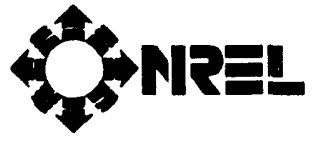

March 1992

Note: HERS - Home Fnergy Rating Systems

EEMs - Energy Efficient Mortgages 
On September 16, 1991, the Solar Energy Research Instltute was deslgnated a natlonal laboratory, and Its name was chan to the Natlonal Renewable Energy Laboratory.

\section{NOTICE}

This report was prepared as an account of work sponsored by an agency of the United States government. Neither the United States government nor an: agency thereot. nor any of their employees. makes any warranty, express or implied. or assumes any legal liability or responsibility for the accuracy, com pleteness. or usefulness of any information. apparatus. product. or process disclosed. or represents that its use would not infringe privately owned rights Reference herein to any specific commercial product, process, or service by trade name. trademark. manufacturer. or otherwise does not necessarily con stitute or imply its endorsement. recommendation, or favoring by the United States government or any agency thereof. The views and opinions of autnor: expressed herein do not necessarlly state or reflect those of the United States government or any agency thereot.

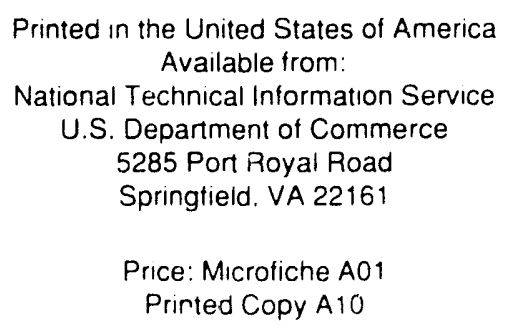

Codes are used for pricing all publications. The code is determıned by the number of pages in the publication. Intormation pertainıng to the pricing codes can be ic and in the current issue of the following publications which are generally avallable in most librarıes: Energy Research Abstracts (ERA): Govern ment Reports Announcements and Index (GPA and 1): Scientific and Technical Abstraci Reports (STAR): and publication NTIS PR 360 avallable from NTIS at the above adress 


\section{Preface}

This collection of papers is a companion volume to A National Program for Energy-Efficient Mortgages and Home Energy Rating Systems: A Blueprint for Action (NREL/TP-261-4677). The Blueprint reports the findings and recommendations of the National Collaborative on Home Energy Rating Systems and Mortgage Incentives for Energy Efficiency ahout a voluntary national program linking energy-efficient mortgages and home energy rating systems.

The National Collaborative wrote A Blueprint for Action and its members and staff prepared the papers in this companion volume, Going National with HERS and EEMs: Issues and Intpacts, which was published by the National Renewable Energy Laboratory.

The Collaborative was formed by the U.S. Department of Energy, in cooperation with the U.S. Department of Housing and Urban Development, as a National Energy Strategy initiative. The Collahorative's purpose was to investigate promoting energy-efficient homes by linking home energy rating systems and energyefficient mortgages.

This volume provides technical documentation for A Blueprint for Action. It consists of 55 technical issuc papers and 13 special papers prepared by the technical advisory committees and some members of the Collaborative Consensus Committee of the National Collaborative. It also contains the bibliography and the glossary written by the members and staff of the National Collahorative.

The valuable contributions of the Collaborative's members, those who served on committees, and staff are acknowledged. Without their dedication and commitment to a challenging task, the accomplishments reflected in this collection of papers would not have been realized. 


\section{Table of Contents}

$\underline{\text { Page }}$

Introduction $\ldots \ldots \ldots \ldots \ldots \ldots \ldots \ldots \ldots \ldots \ldots \ldots \ldots \ldots \ldots \ldots \ldots$

Section 1. Assigned Technical Issues and Special Paper Topics $\ldots \ldots \ldots \ldots$

Section 2. Assigned Technical Issue Papers $\ldots \ldots \ldots \ldots \ldots \ldots \ldots \ldots \ldots \ldots \ldots \ldots \ldots \ldots \ldots$

Home Energy Rating Systems Issue Papers

No. Issue

Author

1 Energy savings and savings cost $\ldots \ldots \ldots$ Ron Hughes $\ldots \ldots \ldots \ldots$

2 Alternative approaches . . . . . . . . . Ron Hughes . . . . . . . . .

Output measures . . . . . . . . . . Ron Hughes . . . . . . . .

Minimum guidelines . . . . . . . . . . . Michael Holtz . . . . . . . 19

Accuracy mechanisms . . . . . . . . . . . Ron Judkoff . . . . . . . . . 23

Fuel neutrality ................ Ron Hughes .......... 25

Occupant behavior . . . . . . . . . . . . Michael Holtz . . . . . . . 27

New and existing home distinction ....... Doug Swartz . . . . . . . . . 32

HERS/EEMs linkage .............. Bill Prindle .......... 34

10) Information guidelines for retrofits $\ldots \ldots \ldots$ Bill Prindle $\ldots \ldots \ldots \ldots \ldots . \ldots . \ldots$

11 Individual vs. group ratings $\ldots \ldots \ldots \ldots$ William Freeborne . . . . . . . . 36

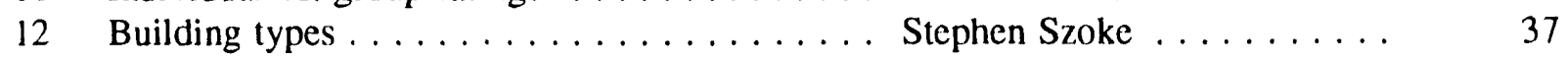

13 Features .................. B. D. Howard ........ 38

14 Level of detail/accuracy ........... Doug Swartz and

Michael Holtz ......... 42

15 Innovative features $\ldots \ldots \ldots \ldots \ldots$. . . . . Ron Judkoff . . . . . . . . . . . 46

16 Minimum required outputs . . . . . . . . Ron Hughes . . . . . . . . . . . 47

17 Linkages to state and local codes . . . . . . Ron Nickson . . . . . . . . . . . . 49

18 Re-rating requirements . . . . . . . . . Steve Baden ......... 50

19 National qualifying threshold $\ldots \ldots \ldots \ldots$ Bill Prindle $\ldots \ldots \ldots \ldots \ldots$

\section{Energy-Efficient Mortgages Issue Papers}

1 Housing affordability and portfolio risk ..... Bill Brewster . . . . . . . . . 52

2 A uniform EEMs program ........... Walter Patterson ........ 53

3 Characteristics of a uniform EEMs program ... Walter Patterson . . . . . . . . . . . 54

4 Debt-to-income ratio adjustment . . . . . . . W Walter Patterson . . . . . . . . 56

5 A uniform EEMs program ........... Bill Brewster .......... 68

6 Appraisal/valuation method .......... Bill Brewster . . . . . . . 69

7 Total energy improvements . . . . . . . . Jane Stockinger . . . . . . . 71

8 Loan-to-value ratio $\ldots \ldots \ldots \ldots \ldots \ldots$. . . . . Jane Stockinger . . . . . . . . . . . . 73

9 Energy efficiency improvement financing . . . . Jim Curtis . . . . . . . . . . . . . 74

10 Delay avoidance ... . . . . . . . . . . Richard Faesy . . . . . . . . . . . 77

11 Information triggers for lenders . . . . . . . Tom Farkas . . . . . . . . . . 78

12 Information triggers for lenders . . . . . Chip Coffay . . . . . . . 8() 
Table of Contents (continued)

Page

Energy-Efficient Mortgages Issue Papers (continued)

13 Escrow alternatives $\ldots \ldots \ldots \ldots \ldots \ldots \ldots$ Jim Curtis $\ldots \ldots \ldots \ldots$

\section{Implementation Issue Papers}

1 Alternative institutional arrangements ..... Donald Buchan . . . . . . 84

2 Estimated costs (existing buildings) . . . . . . F Frank Newbraugh . . . . . . . 86

3 Estimated costs (new buildings) $\ldots \ldots \ldots \ldots$ Richard Tracey . . . . . . . . 88

4 Consumer protection . . . . . . . . . . . Becky Cohen . . . . . . . . 91

5 Resolution of consumer problems . . . . . . Becky Cohen . . . . . . . . 92

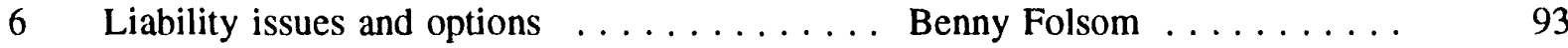

7 Human resources . . . . . . . . . . . . . . Doug Seiter . . . . . . . . . 94

8 Incentives - national system . . . . . . . . Chris Roitsch . . . . . . . . . 96

9 Incentives - localities . . . . . . . . . . . . Russell Duncan . . . . . . . . 99

10 Quality control . . . . . . . . . . . . . Barry Moline . . . . . . . . 100

11 Verification . . . . . . . . . . . . . . . Barry Moline . . . . . . . . 101

12 Mandatory aspects . . . . . . . . . . . Carson Culbreth . . . . . . 102

13 Loan delay avoidance . . . . . . . . . . . Javier Figueroa . . . . . . . 104

\section{A wareness Issue Papers}

1 Generation of information $\ldots \ldots \ldots \ldots$. Rebecca Vories . . . . . . . . 105

2 Informational needs . . . . . . . . . . . . . . Deborah Howard . . . . . . . 107

3 Motivational and technical needs ......... Deborah Howard ........ 109

4 Consumer information needs . . . . . . . . . . Gerry McGowan . . . . . . . 112

5 Technical assistance . . . . . . . . . . . . . . Ranciy Martin . . . . . . . . . 114

$6 \quad$ Trigger points . .............. David Swankin ........ 116

$7 \quad$ Labeling ................. Bill Griffin and

David Swankin ................. 117

$8 \quad$ Labeling $\ldots \ldots \ldots \ldots \ldots \ldots \ldots \ldots \ldots \ldots$ Bill Prindle $\ldots \ldots \ldots \ldots \ldots \ldots$

9 Rater proficiency requirements . . . . . . . Mike Lennon and

David Swankin ........ 120

\section{Section 3. Special Papers}

1 Characteristics, EEMs $\ldots \ldots \ldots \ldots \ldots \ldots$ Perry Bigelow . . . . . . .

Overcoming barriers, EEMs $\ldots \ldots \ldots \ldots$ Perry Bigelow $\ldots \ldots \ldots \ldots$

3 Key harriers to EEMs

Jim Curtis . . . . . . . . .

Farkas Task Force

(Tom Farkas, Doug Swartz,

Ken Crandall, and

Jim Fulkerson) . . . . . . 128

$5 \quad$ Rating model or rating method

Tom Farkas

6 Rater/training qualification

Benny Folsom and 
Table of Contents (concluded)

$\underline{\text { Page }}$

Section 3. Special Papers (continued)

7 Memorandum from risk holders

FNMA, FHLMC, FHA, VA,

FmHA (Charles Fritts) . . .

138

8

Consensus statement Fulkerson Task Force

(Jim Fulkerson, et al.) . . . . .

Implementation .

Ron Hughes

141

10 Cash flow vs. cost-effectiveness . . . . . . . Bill Prindle . . . . . . . . . 146

11 Key barriers to EEMs . . . . . . . . . . . . B Bill Prindle . . . . . . . . . . 147

12 Renewable energy technologies . . . . . . . . Stephen Szoke . . . . . . . . . 150

13 Environmental benefits . . . . . . . . . . . Marika Tatsutani . . . . . . . 157

Appendix A: Members of the National Collaborative $\ldots \ldots \ldots \ldots \ldots \ldots \ldots \ldots$

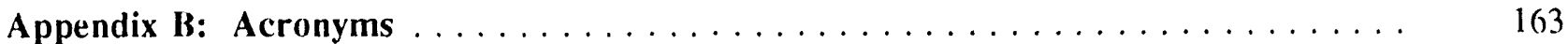

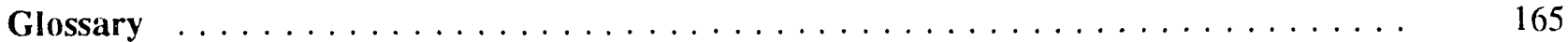

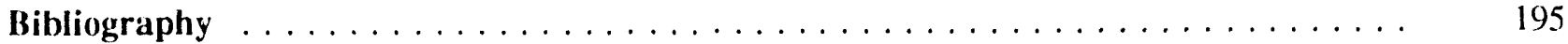




\section{Introduction}

This report contains the collected papers of the National Collaborative on Home Energy Rating Systems and Mortgage Incentives for Energy Efficiency. The Collaborative Consensus Committee (CCC) was responsible for final technical decisions and policy formulations as they are reflected in $A$ Blueprint for Action. The CCC assigned issues it wanted explored to four different Technical Advisory Committees (TACs). The technical papers were advisory to the CCC.

TAC members were nominated by CCC members, reviewed by the Department of Energy management team, and, after selection, approved by the CCC. Technical members in each area of expertise-home energy rating systems, energy-efficient mortgages, implementation strategies, and awareness-were augmented by CCC members interested in attending TAC meetings. Each TAC had two co-chairs. A technical staff member served as the staff co-chair, whose primary responsibility was to interpret the TAC's work to the CCC. The CCC co-chair's responsibility was to ensure that issues the CCC had identified were addressed by the TAC. Collaborative observers also attended TAC meetings and, when recognized by TAC chairs, participated in the proceedings.

The TACs were responsible for providing technical information, options along with their advantages and disadvantages, and, when they thought it was useful or desirable, recommendations.

Although the TACs did not have responsibility for arriving at consensus decisions, the Home Energy Rating System (HERS) TAC was the only TAC to arrive at consensus on each of its 21 issues. These consensus recommendations are reflected in the HERS TAC issue papers.

There are occasional discrepancies between the assigned issue list (approved by the CCC) and the actual issue listed at the top of certain issue papers. This means that the issue paper author rephrased the issue slightly (often for technical reasons), and wrote to the issue as it was stated in the issue paper. These discrepancies were left intact in this report. The assigned issues list, which follows in Section 1, shows the areas of CCC inquiry. The actual issue papers document the issues actually addressed.

Also listed in Section 1 are the topics of the special papers requested by the CCC members later in their deliberations, or assigned by facilitators or staff.

All issue papers and special papers were provided to the CCC for use during their deliberations. However, not all positions recommended by TACs were adopted by the CCC.

Appendix A lists members of the CCC, the Technical Advisory Committees, and the CCC's Steering Committee. A list of staff and official observers also appears in Appendix A. Appendix B contains a list of relevant acronyms. The glossary was compiled by National Renewable Energy Laboratory (NREL) staff members using inputs from CCC and TAC members. Portions of the glossary have been reviewed by CCC and staff members.

NREL staff also compiled the bibliography using a library of papers NREL maintains and from the Collaborative's technical issue papers and special papers. 
Section 1

Assigned Technical Issues and Special Paper Topics 


\section{Assigned Technical Issues and Special Paper Topics}

The Collaborative Consensus Committee (CCC) had numerous technical questions that needed to be answered before the CCC could make policy recommendations about a national program. These issues, listed in this section, were divided among four Technical Advisory Committees comprised of experts in relevant technical fields. The Technical Advisory Committees were assigned to deal with technical issues in the following areas:

- Home energy rating systems (HERS)

- Energy-efficient mortgages (EEMs)

- Implementation strategies and impacts (Implementation)

- Marketing, training, and promotion (Awareness).

In addition to the issue papers, Collaborative members wrote special papers to address concerns that arose during the CCC's consensus-building discussions. CCC members, facilitators, and staff requested that these special papers be prepared by individuals or ad hoc task forces. This section lists the topics of these special papers.

\section{Assigned Issues}

\section{HERS Technical Advisory Committee}

Technical experts in home energy rating systems, energy auditing, energy-efficient design, and retrofits.

1. What studies show that home energy rating systems, where they have been applied, actually result in saved energy in residential buildings? Saved operating costs? If system did not result in energy savings, why not? If energy savings were realized, what were the contributing factors? (Ron Hughes)

2. Should alternative rating approaches be allowed, e.g., performance, prescriptive, utility bills, pass/fail, scaled, etc.? (Ron Hughes)

3. Upon what measures should the rating he based (Btu/year, Btu/ft ${ }^{2} / \mathrm{yr}, \mathrm{Btu} / \mathrm{ft}^{2} / \mathrm{DD}, \$ 1$, etc.)? Absolute measures, comparative measures, dollar savings, etc. (Ron Hughes)

4. What are the minimum guidelines to ensure technical credibility, uniformity, and allowance for local variations? (Michael Holtz)

5. What are the mechanisms for determining the accuracy of a rating system? (Ron Judkoff)

6. How does HERS industry deal with fuel neutrality? Can we develop a rating system that is not biased by fuel? If yes - what does TAC recommend? (Ron Hughes)

7. What information on behavioral patterns provides useful insights into how a HERS system might incorporate occupant behavior? (Michael Holtz)

8. How should HERS distinguish between new and existing homes? (Doug Swartz)

9. To what extent should there be linkage hetween HERS and EEMs? (Bill Prindle) 
10. What information should HERS guidelines require vis-à-vis retrofits? Should the HERS give a dollar cost and saving by component of proposed retrofit? (Bill Prindle)

11. Are ratings required for each building, or can group ratings be permitted? (or subsets of ratings) (William Freeborne)

12. What building types should be included in HERS? (Stephen Szoke)

13. What features should be included in HERS? (Bion Howard)

14. What level of detail/accuracy is necessary? (Doug Swartz)

15. How do we include innovative buildings/features in the HERS, yet keep it from being too cumbersome? (Ron Judkoff)

16. What are the minimum required outputs from HERS tools? (Ron Hughes)

17. To what extent should HERS be linked to state and local codes? (Ron Nickson)

18. Under what conditions is re-rating required? (Steve Baden)

19. Should there be a national threshold for qualifying for an EEM? If not, how will local thresholds be decided? (Bill Prindle)

\section{EEMs Technical Advisory Committee}

Technical experts in mortgage financing, real estate, appraisal, housing, law, and administrative requirements.

1. What data or analyses do we have showing that energy-efficient mortgages increase housing affordability? (e.g., if increased mortgage expense is offset by decreased operating expense, how does the home owner come out ahead financially?) Do any field data or analyses demonstrate how the homeowner benefits? How many more households might qualify for housing? What analyses have been done (or need to be done) on the impact of energy-efficient mortgages on portfolio risk? (Bill Brewster)

2-5. Is it desirable to have a uniform EEM program and, if so, what are its characteristics? What underwriting rules should apply to EEMs? What should qualifying ratios be? Should a ratio stretch or a PITI + E approach be used? If the debt-to-income ratios are increased for mortgagees having energy-efficient housing, what should the ratios be? One specific amount or scaled to reflect the building's energy performance? or income level? What should the ratios be for given household incomes and/or building energy performance levels? (Walter Patterson and Bill Brewster - 2 papers)

6. What would a uniform appraisal/valuation method be? How does one measure the value of energy-efficiency improvements? How should they be accounted for in the appraisal process? What data or analyses have been done (or are needed) on the impact of energy improvements on resale value of house? (Bill Brewster)

7. For an existing home, what should the total amount of energy improvements be? (Jane Stockinger) 
8. What should the total loan-to-value ratio of EEMs be? (Jane Stockinger)

9. For existing mortgages, how can energy-efficiency improvements he financed as part of the mortgage? (Jim Curtis)

10. How can the EEM process be designed to avoid delay in the process? (The EEMs TAC will deal with the mechanics of what can be done; the IMP TAC will deal with who should do it.) (Richard Faesy)

11-12. What kind of information is needed by lenders to trigger an energy efficient mortgage? What basic quantities do the mortgage lenders need from the HERS? (Chip Coffay - lender perspective; Tom Farkas - HERS perspective)

13. What alternatives to escrow arrangements can be considered? Would a trustee system for fees, escrow accounts, and savings guarantees be beneficial? (Jim Curtis)

14. How might inducements (such as rebates) be treated within an EEM process? When would such inducements be available? (Ken Crandall)

\section{Implementation Technical Advisory Committee}

Technical experts in utility programs, state energy programs, home inspection, remodeling, impact assessment, management analysis, cost/benefits analysis, and legal analysis.

1. What alternative institutional arrangements are possible to implement HERS/EEMs? For example, should HERS/EEMs be delivered through state programs? Through utilities?

What alternative strategies at the federal, state, regional and local level are required to implement a uniform national energy-efficient mortgage program linked to a voluntary home energy rating system process? Whose participation is required at each level?

(Consider the whole range of HUD and USDA community development and housing programs: those of local Home Builder Associations (HBAs); local government housing, code enforcement and community planning and development programs, better housing organization and other social service agency activities; housing and planning programs of regional councils of government (COGs); utility audit and DSM/rebate programs; and the roles of state community affairs, housing and energy agencies, national trade and professional associations, and federal regional offices.)

How can the strategies identified and existing utiiity, HBA, local or state government energyefficiency mortgage and home energy rating system programs be effectively linked? (Don Buchan)

2. (With respect to existing huildings.) What are the estimated costs to each actor associated with implementing the various alternatives? What existing data or other measures were used to make these cost estimates? How might these costs be financed? What might the financial impact be on home huyers? state budgets? local budgets? taxpayers? utility rates and stockholders? others? (e.g., who will win and who will lose, if anyone?) 
What empirical evidence is there on which to base estimates of the costs involved in implementing a nationwide HERS/EEMs system? What financial resources would be necessary to implement HERS/EEMs? What are programs going to cost at local, state, and federal levels? (Frank Newbraugh)

3. (With respect to new buildings.) Same question as 2. (Richard Tracey)

4. What would consumer impacts be? What are consumer protection issues, and how can they be prevented? (Rebecca Cohen)

5. How can consumer protection issues be resolved? (Rebecca Cohen)

6. What are liability issues and options? (Benny Folsom)

7. What human resources would be necessary for implementation? (Doug Seiter)

8. What role might the states play, and what incentives might be needed to encourage states to participate in a national system or program? (Chris Roitsch)

9. What incentives are needed to encourage local participation? (Russell Duncan)

10. What are the actions necessary for quality control, who would carry them out, and what would be the estimated costs? (Barry Moline)

11. Who should verify the adequacy/accuracy of building energy predictive tools? (Barry Moline)

12. What aspects of a combined HERS/EEMs program might be mandatory? (Doug Culbreth)

13. What are the roles of the various participants in avoiding delay in the loan approval process? (related to EEMs Issue \#10) (Javier Figueroa)

\section{Awareness Technical Advisory Committee}

Technical experts in marketing, advertising, consumer protection, training, needs assessment, and education.

1. Generation and distribution of HERS/EEMs information for consumers and shelter industry audiences. (Rebecca Vories)

2. What are the informational needs of the following groups? (Deborah Howard)

- Mortgage lenders

- Appraisers

- Inspectors

- Real estate agents

- Code officials

- Energy auditors

- Builders

- Remodelers /retrofitters

- Dealers

- Manufacturers of manufactured homes 
- Product suppliers and manufacturers

- Organized consumer advocacy groups

- Utilities

- Organized energy and environmental a dvocacy grouns

- Regulators

- Relocation companies

- Real estate attorneys

- Homeowner associations

- State and local executives and legislative bodies

3. What are the motivational needs of the following groups? (Refer to groups listed for item 2). What are the technical needs of the following groups? (Refer to groups listed for item 2) (Deborah Howard)

4. What are the information and education needs of consumers and how can they best be met? What are the components of an objective consumer education program for HERS/EEMs? (Gerry McGowan)

5. What provision will be made for technical assistance? (Randy Martin)

6. What are the best "trigger points" for awareness in the new and resale home sales processes? How can these be activated? (David Swankin)

- Time of contract signing

- Time of mortgage application

- Time of listing home

- Time of home inspection

- Time of multiple listing

- Utility bill insert

- Real estate agent promotional mailings

- Time when consumers first consider buying, selling, or refinancing home

- Time of remodeling, retrofitting, or major repair

- Time of refinancing for any reason

7-8. What types of labeling will be best in terms of helping promote awareness? What type of labeling, if any, will be best in terms of helping promote awareness? What are the criteria for evaluating a "good" label? (Bill Griffin/David Swankin) What labeling should be requied for a home energy rating? (Bill Prindle)

9. What proficiency requirements should there be for raters and anyone making calculations on retrofits? (Mike Lennon)

\section{Special Papers*}

Perry Bigelow Characteristics of an energy-efficient mortgage program

*Special papers were produced on request of Collaborative members and staff. Their purpose was to address pertinent issues arising from the CCC discussion. 
Perry Bigelow

Jim Curtis

Farkas Task Force

Tom Farkas

Benny Folsom

Charles Fritts

Fulkerson Task Force

Ron Hughes

Bill Prindle

Bill Prindle

Stephen Szoke

Marika Tatsutanti
Overcoming the barriers and disincentives to the purchase of energyefficient homes with energy-efficient mortgages

Barriers to energy-efficient mortgages

Is a single "rating method" necessary for uniformity?

What is a rating model or rating method? Is it necessary for the CCC to select one to establish uniformity?

Rater training/qualifications

Special papers from the lenders' caucus

Special paper on a national benchmark

What could an infrastructure for a national implementation and administration of a HERS/EEM program look like?

Cash flow vs. cost-effectiveness

Key barriers to the wider use of EEMs for retrofitting existing homes

Renewable energy technologies for home energy rating systems and energy-efficient mortgages

Environmental benefits of a national HERS/EEMs program 
Section 2

Assigned Technical Issue Papers 
Issue Paper No.: $\quad 1$

Name of TAC: ${ }^{1} \quad$ HERS

Contact person: $\quad$ Ron Hughes, HERS Industry (Energy Rated Homes of America)

Date of issue paper: October 4, 1991

Title of issue: What studies show that energy improvements, where they have been applied, actually result in saved energy in residential buildings? Saved operating costs? If not, why not? If energy savings were realized, what were the contributing factors?

Summary of analysis already completed: Of the studies reviewed, comprehensive energy improvements have resulted in savings of energy and operating costs ranging from $9 \%$ to $32 \%$ in single-family houses and average $26 \%$ in multifamily structures.

Individually, adding insulation showed an average savings of $21 \%$; improving heating systems resulted in $11 \%$ to $13 \%$ savings; and reducing leakage in the air distribution system averaged $22 \%$ savings, hut ranged up to as high as $41 \%$ !

The lower range of savings from energy improvements appear to be the result of: (1) improvements to houses that were fairly efficient privi to improvements, (2) poor installation of improvement measures, and/or (3) lower-income families who, after weatherization, chose to condition (heat) and occupy more rooms of the house than they previously could afford to condition.

Contributing factors to the extent of energy savings included (1) the extent of the energy improvements, (2) the particular improvements chosen, (3) the quality of the installations, (4) lifestyle changes that resulted, and (5) the procedures employed to evaluate savings.

Research still needed: None

Background text and references: See attachments

'Technical Advisory Committee 


\section{Attachment to Issue Paper No. 1 References Reviewed}

Barnett. Cole and Thor, Phil. June 1990). Comstruction Cost Analysis Residemtial Comstruction Demonstratiom Project, Cycle II. Bunneville Power Administration, Portland, Oregon.

Baylon, David, Boh Davis, Ian Brown, Mike Kennedy, Mike Lubliner, and Steve Onisko. Ecotope. March 1991. Mamufactured Homes Thermal Analysis and Cost Effectiveness Report. Bonneville Power Administration, Portland, Oregon.

Brown, Evan. May 1991. Energy Raled Homes of Arkansas. Research Report on Energy Savings from Weatherization Measures. Prepared for the Arkansas Department of Human Services, Little Rock, Arkansas.

Carlsmith, Roger S., William U. Chandler, James E. McMahon, and Danilo J. Santini. January 1990. Energy Efficiency: How Far Can We Go? Oak Ridge National Laboratory, Oak Ridge, Tennessec.

Cohen, S. D., C. A. Goldmen and J.P. Harris. February 1991. Mecasured Energy Savings and Economics of Retrofitting Existing Single-Family Homes: An Update of the BECA-B Database. Lawrence Berkeley Laboratory, Berkeley, California.

Cohen, Sam and Goldman, Chuck. July/August 1991. Between Two Decades: Weatherization Report Card. Home Energy, Vol. 8, No. 4, pp. 30-36.

Davis, Bruce E. September 1991. The Impact of Air Distribution System Leakage on Heating Energy Consumption in Arkansas Homes. Prepared for the Arkansas Energy Office by Home Comfort, Fayetteville, Arkansas.

Goldman, C.A. May 1988. Retrofit in U.S. Multifamily Buildings: Energy Savings, Costs, and Economics, Volume 1. Lawrence Berkeley Laboratory, Berkeley, California.

Hellman, Marc. July 1991. Investigation into Electric Utility Incentives for Acquisition of Conservation Resources. Oregon Public Utility Commission.

Howard, Bion and Prindle, William. July 1991. Better Building Codes for Energy Efficiency. Alliance to Save Energy, Washington, DC.

Koomey, Jonathan G., Celina Atkinson, Alan Meier, James E. McMahon, Stan Boghosian, Barhara Atkinson, Isaai Turiel, Mark P. Levin, Bruce Nordman, and Peter Chan. July 1991. The Potential for Electricity Efficiency Improvements in the U.S. Residential Sector. Lawrence Berkeley Laboratory, Berkelcy, CA.

Lessne, Greg J., PhD. Novemher 1987. Evaluation of Energy Conservation Assistance Programis. University of Rhode Island, Providence, Rhode Island.

Nichols, A.K. and Belzer, D.B. November 1988. Estimation of Building Sector Energy Savings Potential from OBCS Funded Research: A Technical Support Document. Pacific Northwest Laboratory, Richland, Washington. 
Pratt, R.G., C. C. Conner, M.K. Drost, N.E. Miller, B.A. Cooke, M.A. Halverson, B.A. Lehaun, R.G. Lucas, J. Jo, E.E. Richman, K.G. Ritland, W.F. Sandusky, M.E. Taylor, and S.G. Hauser. December 199(). Significant ELCAP Allalysis Results: Summary Report. Pacific Northwest Lahoratory, Richland, Washington.

Rogers, Beth. November/December 1991. Manageable Energy Management. Public Power, Vol. 49, No. 6, pp. 20-25.

Rosenfeld, Arthur H., Celina Atkinson, Jonathan Koomey, Alan Meier, and Rohert Mowris. LBL. February 1991. Policy Implications of Greenhonse Warming. "A Compilation of Supply Curves for U. S. Buildings," Chapter 3: Residential and Commercial Energy Management. National Academy of Science. Lawrence Berkeley Laboratory, Berkeley, California. 
Issue Paper No.: $\quad 2$

Name of TAC: $\quad$ HERS

Contact person: $\quad$ Ron Hughes, HERS Industry (Energy Rated Homes of America)

Date of issue paper: Septemher 3(), 1991

Title of issue: $\quad$ Should alternative rating approaches ha allowed?

Recommendations (if any) (include majority/minority): Yes. One performance-based, (1-1(\%) rating scale would he defined for each jurisdiction or locale according to climate and construction practices. A variety of certification, prescriptive, and code approaches could then be allowed as long as they:

1. Can be related to the scale in a technically credible manner, and

2. Meet or exceed the efficiency threshold for an EEM on the siale of the subject jurisdiction or locale.

Advantages:

- Standardization of haseline scaled approaches.

- Allows for local autonomy; tailored to local needs.

- Most exciting, scaled, state-sponsored HERS use a ()-100 point scale.

- Diversity of certification and prescriptive approaches that can be approved.

Disadvantages:

- Certification programs number in the hundreds.

- Alternate approaches vary widely in protocols and standards and may require change.

- Alternate approaches encourage "gaming" to most easily qualify a particular housc.

- Quality control of many approaches will be more difficult.

Summary of analysis already completed:

- Review existing HERS programs.

- Discussion within HERS TAC, HERS industry, and representatives of the National Association of State Energy Officials.

Alternative solutions with advantages and disadvantages: No-allow only one approach.

Advantages:

- Lenders and national audiences have only to deal with a single system.

- More resources would be devoted to a single approach, perhaps ending up with a stronger more effective program.

\section{Disadvantages:}

- Most, if not all, existing programs would be ruled out.

- Resources are not always available for conversion from existing to new approach.

- New approach could he a lemon if not already in place and tested.

- Political opposition. 


\section{Research still needed (if any):}

- How to quality control a variety of approaches.

- Define 0 and 100 points on the scale for uifferent states and climatic regions.

- How/where are certification/prescriptive programs approved? Recommendations: On the state level.

Background text and references: See Glossary. 
Issue Paper No.: $\quad 3$

Name of TAC: $\quad$ HERS

Contact person: $\quad$ Ron Hughes, HERS Industry (Energy Rated Homes of America)

Date of issue paper: October 18, 1991

Titı: of issue: $\quad$ Upon what measure should the rating be based (Btu/yr; Btu sq ft/yr; Btu/sq ft/DD; etc.) e.g., absolute measures, comparative measures, dollar savings, etc. ("Based" is interpreted to mean the "fundamental" physical quantity which is the primary technical output of a HERS calculational tool.)

Recommendations (if anv) (include majority/minority): Btu/yr; such a quantity may, or may not, he seen by the consumer; however, it is easily convertible to all other commonly used energy units and normalization paranıters.

\section{Advantages:}

- Establishes a performance measure that is not time dependent.

- Easily converted into MBtu/yr and dollar costs.

- While possibly confusing, may or may not be seen by consumer.

- Diversity of certification and prescriptive approaches that can be approved.

Disadvantages:

- Btu/yr is not common terminology ir the marketplace.

Alternative suiutions with advantages and disadvantages:

Rating based on dollars, not energy.

Advantages/disadvantages: Dollars are certainly the bottom line, and purchased energy is easily determined from utility bills-but it is difficult to determine the impact of lifestyle. In addition, new houses have no metered history, fuel prices fluctuate, and different fuels are not always available.

Rating includes demand charges.

Advantages/disadvantages: Varies. Unpredictable. Instead include demand charges in the utility rate schedules when predicting energy costs-not when rating efficiency.

Summary of analysis already completed: Discussions with HERS industry concerning what works, why chosen, and what could he better.

Research still needed (if any):

1. Research alternatives for normalizing.

2. Where is the energy measured? Source energy or purchased energy? Is this question part of the national HERS protocols or a local decision?

3. Do climate issues get incorporated into the fundamental unit or more appropriately into the definition of the rating scale end-points?

4. Who sees what numbers, and where do they appear on labels, stickers, or documentation? 
Issue Paper No.: 4

Name of TAC: $\quad$ HERS

Contact person: $\quad$ Michael J. Holtz, Architectural Energy Corporation

Date of issue paper: September 12, 1991

Title of issue: What are the minimum guidelines to ensure technical credibility, uniformity, and allow for local variations?

Recommendations (if any) (include majority/minority): As a starting point, the following are suggested as the minimum guideline topics for inclusion in the National HERS/EEM Collaborative's Blueprint for Action:

- Uniform scale of efficiency (numerical rating) $(2,3,6)$

- Procedure for testing and certifying the accuracy of rating tools (including alternative compliance method) $(5,14,15)$

- List of minimum rated features $(7,8,10,12,13)$

- Home energy labeling requirements (16)

- Linkage of energy rating to energy-efficient mortgages $(9,16)$

- Procedures for monitoring, quality control, and evaluation (5)

- Procedure for data collection and reporting of rated houses (9)

- Definition of or procedure for determining minimum efficiency level for HUD, VA, and FMHA insured or guaranteed loans $(17,19)$

- Other $(1,18)$.

Summary of analyses already completed: The National HERS/EEM Collaborative has been formed to develop a "Blueprint for Action" outlining the parts that various organizations should play in moving forward a national program of HERS/EEMs. This Blueprint for Action will consist of a number of guidelines that organizations developing, implementing, operating, and maintaining HERS/EEMs should adhere to in order to ensure uniformity and credibility. At issue is the minimum set of technical guidelines required to ensure credibility, uniformity, and local/state flexibility.

The National HERS/EEM Collaborative cannot and should not tell organizations involved in home energy ratings and energy-efficient mortgages what to do. Rather, it should develop/detine a minimum set of guidelines that, if followed, would constitute a technically defensible, uniform HERS/EEM process. The issue before the National HERS/EEM Collaborative and this HERS Technical Advisory Committee is what are the minimum set of guidelines that should be identified, developed, and included as part of the Blueprint for Action.

The identification of guideline areas should be driven by the existing and proposed legislation that has required or will require consideration of energy efficiency in the home financing process, or the establishment of a national voluntary home energy rating system. Additionally, those areas deemed essential by the HERS/TAC to ensure an effective technical basis of a HERS should be included in the guidelines.

The issue paper, prepared hy the Alliance to Save Energy (attached), briefly describes some of these topics. 
Alternative solutions with advantages and disadvantages:

Advantages:

- Establishes a hasic set of guidelines for ensuring technical credihility and uniformity of HERS/EEM.

- Preserves state and/or sponsoring organization's prerogative to adopt and modify HERS/EEM to meet locial needs and requirements.

Disadvantages:

- May leave areas open that could cause confusion or misinterpretation in the future.

Research still needed (if any): Research needs for the guideline topics are presented in other HERS TAC issue papers related to these topics. 
Attachment to Issue Paper No. 4

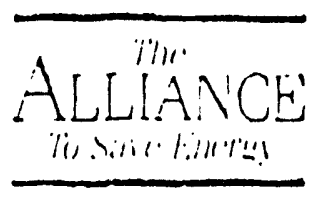

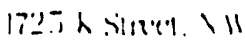

Silits 11.1

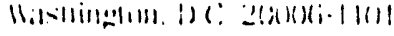

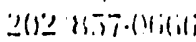

lin: 2(1) :3311-917ns

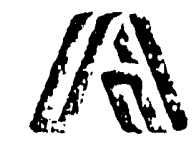

COLIRBORATIVE CONSENSUS COMMITIEE

ON ENERGY ENFICIENT MORTGAGES AND HONE INERGY RATINGS

HERS MECENICAL ADVISORY COKMITTEE

POTENTIRI ISSUES FOR DISCUSSION

1. A unifozm scale of exergy exficiency -- Effective naticnal guidelines for home ene gy ratings must establish a common scale on which all howes can be compazed. Such a scale must have a technical basis and a relatively simple reporting index. Ey:ampies of cechnical bases include BTU/souare foot/degree day and similar thermal load measuzes, percentage achievement cf an cptimal efficiency level, or percentile ranking of a given home compared to the entire stock in its coographic azea. peporting indices include points systems (uswaily 1-100), stars, colors, and cthers. It is also possible for the technical scale and the reported incex to be the same.

2. A comon threshold for energy efficiency -- For whatever scale is adop=ed, HERS guidelines zust establish threshold criteria for detining homes as energy efficient. These criteria are essertial for gualizying houes for EEM incentives. Given the variety of rating systems and home design appreaches now in use, alternative compliance methods will be necessary.

3. Testing and certifying the accurasy of rating tools - The guidelines must also define criteria for certifying the accuracy of home energy rating tools in predicting both the mil locds and entirgy savings srom retroits. Other guidelines for rating tools nay nead to be set, such as methods for calculating the interaction of retrofits as well as their economic and Enancial pezformance. once these guicelines are set and in place, there must te an admiristrative procedura and sectetariat established to perform the testing and certification protocols. 
4. Home features included in the rating -- Mary rating systens evaluate onIy the thermal efficiency of the buiding envelope. others factor in tVAC equipment efficiency. other physical Eeacures that way be considezed in home encrgy zatings inciucie:

- sir irilitration

- Domestic hot water

- Thermal Mass

- Home automation and contzol systers - mo

- Passive solar fearures

- Active solar and other on-site renewable sources

- Iighting.

- Applances - no

The guidelines must be specific about the components of total hore energy consumption that are to be included in the calculation of etticiency on the common scale.

5. Home enezgy labeling requirements -- National home enezgy rating guidelines must address the issue of labeling. The iebel is what the consumer as wcll as real estate and other professionals see. It must contain infomation that is simple enough for consumers to understand and complete enough for pzotessionals to use. The simplest possible might contain only a scale index rating; yet appraisers and others colld benelit from more descriptive information on energy features such as envelope insulation values, equiprent efficiencies, etc. snothcr possible label item is the estimated energy cost for the home. The guidelines process must set minimum labeling zeouizements.

5. Linkage of energy ratings to energy efficient mortgages -- The CCC process is ciesigned to develop gridelines for both HERS and EEMs. The HERS guidelines thus should support and enable the use of EEMs. Eirst ind foremost, the yeRs guidelines must set the tr. =eshold criter:a for energy efficiency that calify homes for EEM underwriting incentives. other issues that cone up in this context include:

- guidelines for analyzing vetrolits for EDSs

- determining allowable retrofit measures

- guidelines for economic analysis of retrofits

- guidelines for appraisal celculations for retrofits

- guidelines for affordability inpacts of retrofits

7. Honitoring; quality co:trol and evaluation -- The IIERS TAC should discuss these three iscues, addressing ouestions suci as: should rating systems operating under federal gididilines be required to monitor pezozmence of bomes they decl with nsing ceziain protocols? Sinculd a Iederally-funded monitoring etsort be created? Should spot zield Inspectiors of zating systems be required? should there be an overall evaluation of the exfectiveness of the gridelines? 
Issue Paper No.: 5

Name of TAC: HERS

Contact person: $\quad$ R. Judkoff, National Renewable Energy Laboratory

Date of issue paper: Octoher 1,1991

Title of issue: What are mechanisms for determining the accuracy of a rating system?

\section{Recommendations (if any) (include majority/minority):}

1. Develop a qualification procedure for HERS calculation methods using software-to-software comparative tests.

2. Use a combination of short-term monitoring tests, long-term submetering, and long-term utility bill monitoring to provide feedback for improvement of the ratings.

\section{Summary of analyses already completed:}

A combination of four techniques can be used to:

1. Prescreen HERS software (calculation methods)

2. Periodically test HERS data gathering and input adequacy

3. Provide feedback on the average accuracy of occunancy behavior assumptions

4. Provide statistical feedback on the comparability of HERS energy use projections to utility hill data.

The basic techniques for assessing the accuracy of a home energy rating system are described below:

\section{Software-to-software comparisons:}

This is a prescreening approach that could be used to qualify HERS calculation methods for use under the national HERS umbrella. This involves: (a) defining representative prototype test buildings with various types of energy features; (b) simulating the energy performance of those test buildings with several detailed state-of-the art simulation programs such as DOE2. BLAST, SERIRES, etc.; (c) comparing the predictions from the state-of-the-art simulation programs to those obtained with candidate HERS calculation methods; and (d) establishing the degree of accuracy (degree of agreement with the detailed programs) required for a HERS to "pass" the test.

\section{Short-term energy monitoring (STEM) test:}

This is a spot-check method that could be used on a random, small statistical sample of all rated huildings. This involves: (a) vacating the building; (b) installing instrumentation; (c) taking data for 3 days and nights; (d) removing instrumentation; (c) extrapolating to annual energy performance based on the measured building parameters and historical weather data; and (f) comparing extrapolated performance to the prediction obtained from the HERS using the same weather data as for the extrapolation. This method eliminates "occupant effect" as an unknown and would he most useful for detecting inadequacies in the data gathering and input portion of HERS. A slight variant of this method used in conjunction with submetering could also be used to directly measure the magnitude of "occupant effect." This would be done hy using actual weather data taken at the site 
with a temporary weather station for the extrapolation, and then comparing the extrapolated energy usage with no occupant effect to actual submetered energy usage with occupant effect.

\section{Long-term submetering tests:}

This is a continuous check method that could only be used on a very small number of rated buildings. This would involve: (a) submetering the building; and (b) comparing the submetered measured thermal performance of the building to that predicted by the HERS. This method does not distinguish occupant effec $\hat{\imath}$ unless it is lived in conjunction with method \#2. This method is more accurate than utility bill data because uncertainties caused by shared fuel end uses are eliminated hy the submetering. The primary use of this method would be to fine tune assumptions about average occupant behavior in HERS.

\section{Long-term utility bill monitoring tests:}

This is a continuous check that could he used on almost all rated buildings. It would involve comparing energy usage inferred from utility bills to that predicted by the HERS. The primary use of this method would be an overall check of HERS accuracy. This method does not directly help to improve HERS since it is impossible to distinguish between calculational, data gathering, and occupant related sources of error with this method alone.

Each of these four methods has pros and cons. However, a practical, and economical Q/C process can be developed for HERS by using a combination of all four of these methods.

\section{Research still needed (if any):}

1. A piocedure will need to be developed to test the accuracy of HERS calculation software according to method \#1 above.

2. The exact protocols and monitoring requirements will have to be established for developing methods $\# 2$, \#3, and \#4 into a coherent quality control and feedtuck program. 
Issue Paper No.: 6

Name of TAC: $\quad$ HERS

Contact person: $\quad$ Ron Hughes, Energy Rated Homes of America

Date of issue paper: October 18, 1991

Title of issue: $\quad$ How does the HERS industry deal with fuel neutrality?

Recommendations (if any) (include majority/minority): Fuel neutrality will be addressed by requiring certified HERS tools to produce accurate energy use and cost projections. Other details of fuel source issues should be decided at the local level.

Summary of analyses already completed: Most scaled rating systems (Energy Rated Homes [ERH] of AK, AR, VT, RI, MS, VA, and west TX plus AzHERS of AZ and CHERS of CA) rate the overall house on the same 0-100 point scale-whether gas heated or electric heated. Fuel neurality is addressed at the heating system in two different ways (ERHA with potential savings "points" and CA, AZ, and Ft. Collins, $\mathrm{CO}$, with source energy) with similar net results.

When comparing heating plants, the ERH approach compares gas to gas, electric to electric, and oil to oil, etc. when awarding heating system points towards the overall rating score. The highest efficiency gas furnace $(97 \%)$ receives the same "points" as received by the highest efficiency electric, ground-coupled heat pump (300\%).

AzHERS, CHERS, and Colorado's HERS use "source energy" to achieve fuel neutrality-taking into account everything between the natural resource and the usable form. A multiplier of about $33 \%$ is used to adjust the efficiency of electric heating equipment. (Gas is considered usable energy in its natural form and has no such multiplier.) For instance, the efficiency of electric resistance baseboard heaters is reduced from $100 \%$ (at the plug) to $33 \%$ (from the power plant source); a ground-coupled heat pump drops from $300 \%$ to $100 \%$. Instead of $3,413 \mathrm{Btu}$, a kilowatt hour ( $\mathrm{kWh}$ ) is considered equivalent to $10,240 \mathrm{Btu}$ in Arizona and 10,600 Btu in Colorado. The difference is due to a different mix of electric generation sources (coal, hydro, nuclear, etc.) - which will vary in different areas.

Research still needed (if any): None.

Other approaches: Ft. Collins, $\mathrm{CO}$, uses two different $0-100$ point scaies and rating designations for gas and electric heated houses ( $\mathrm{G}$ and $\mathrm{E})$. The end-points of the scales range from 6 to $180 \mathrm{KBtu} / \mathrm{sq} \mathrm{ft} / \mathrm{yr}$ for gas and from 3 to $90 \mathrm{KBtu} / \mathrm{sq} \mathrm{ft} / \mathrm{yr}$ for electric heated homes. In effect, this is a multiplier of $50 \%$ for electric heated houses. While this works for $\mathrm{Ft}$. Collins, with only two fuel sources, it would be confusing to have a different scale for a variety of fuel sources-electricity, natural gas, propane, coal, oil, etc.

Austin's Energy Star rating compares electric to electric and gas to gas as it rates the house by comparing it to the local energy code. The code is fuel neutral in thermal requirements of the house. An energy label accompanies the rating and features only the dollar cost.

Another code-based approach being explored by American Electric Power uses Manual $\mathbf{J}$ to analyze the energy consumption of a house "as is" compared to "if built" to the Model Energy Code (or other standard), irrespective of fuel source. A rating is derived by dividing the energy use of "as is" by "as if." This uncouples the fuei source and does not imit the maximumi rating. 
Not all approaches are fuel neutral. Certification programs, for instance, are sometimes designed not to be fuel neutral-they are designed to be fuel specific, giving extra credit or lowering thermal standards for a particular fuel type or product. 
Issue Paper No.: 7

Name of TAC: $\quad$ HERS

Contact person: $\quad$ Michael J. Holtz, Architectural Energy Corporation

Date of issue paper: October 7, 1991

Title of issue: What information or behavioral patterns provide useful insights into how a HERS might incorporate occupant behavior?

Recommendations (if any) (include majority/minority): To ensure consistency and credihility, a HERS must "rate the home using standardized occupancy assumptions." Those features of the home under control of the occupants, such as thermostat set points, removable lights and appliances, a whole-house fan, and water heater set point, must be fixed in the rating tool so that the rating cannot be raised or lowered depending on the values assumed for those occupant-related factors. The HERS tool must incorporate coverage estimates for lifestyle parameters in order to account for average occupant hehavior.

Summary of analyses already completed: Numerous studies have heen completed, as noted in the attachment, which show the impact of occupant lifestyle on residential energy consumption. These studies indicate the energy use can vary by a factor of 2 or 3 for different families living in identical houses.

\section{Alternative solutions with advantages and disadvantages:}

\section{Advantages:}

- Provides a consistent methodology for accounting of the energy impacts of occupant-related design features

- Eliminates the ability of the rater to "game" the rating by manipulating occupant-related design features.

Disadvantages:

- Rating does not reflect energy use and costs of the actual occupants of the home.

Research still needed (if any):

- Heating and cooling set points hy state

- Water heater set point

- Internal gains hy lights, appliances, and people hy state and/or housing type

- Operating occupancy schedules

- Number of people assumptions - hy number of hedrooms or square feet of floor area. 


\section{Attachment A to Issue Paper No. 7 \\ Partial List of Applicable Studies}

EPRI Report CU-7069, Energy Use and Changing Lifestyles; Lawrence Berkeley Laboratories; Berkeley, California; November 1990.

EPRI Report EM-5908, Residential Customer Preference and Behavior: Market Segmentation Usir?g CLASSIFY (TM) by National Analysts, SRC, and QEI, Inc.; March 1989.

Giadhart, P., Morrison, B., and Zuiches, J.; Energy and Families; East Lansing, Michigan; Institute for Family and Child Study; Michigan State University Press; 1987.

EPRI Report CU-6487, Residential End-Use Energy Consumption: A Survey of Conditional Demand Estimates by Cambridgc Systematics, Inc. of Berkeley, California, and Regional Economic Rescarch, Inc. of San Diego, California.

Kempton, W., and Neiman, M., eds; Energy Efficiency, Perspectives on Individual Behavior, Washington, DC: American Council for an Energy-Efficient Economy; 1987.

Energy Information Administration, Residential Energy Consumption Survey 1983/84 (RECS); United States Department of Energy, Washington, DC; 1987. 
Ittachment B to lssue Paper No. 7

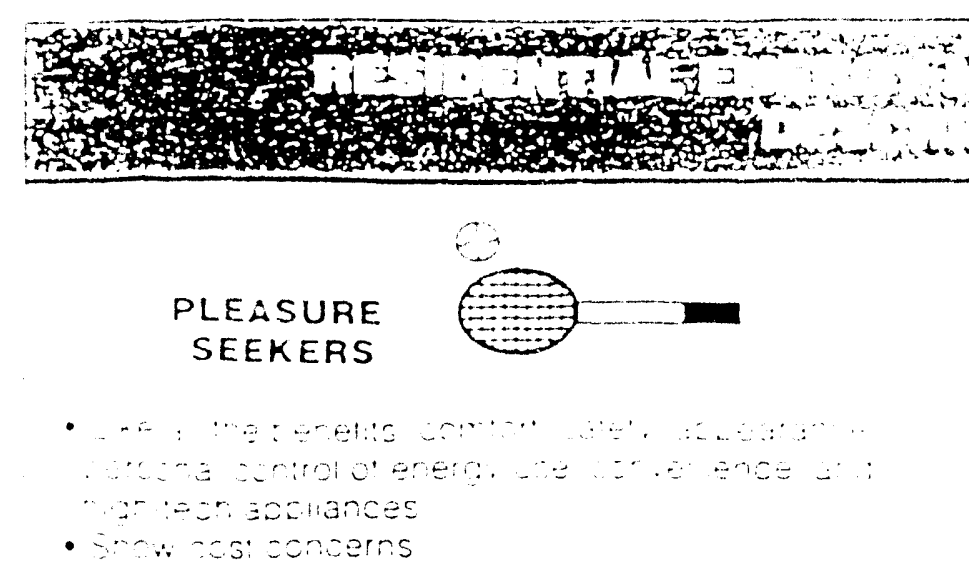

\section{APPEARANCE DONSCIOUS}

- Exhibit appearance and salely concerns

- Less likely 10 monitor energy use

LIFESTYLE SIMPLIFIERS
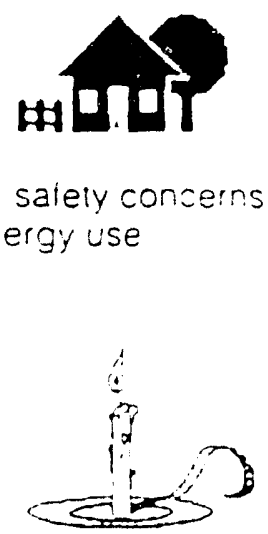

- Snow ess concerr. acoul mosi neesenenel:

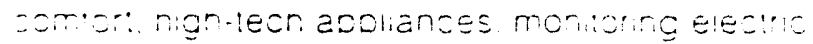

-sage zersonal coniro acherience or

azezerarice ci nome

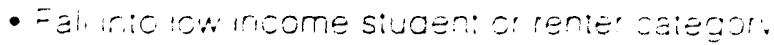

RESOURCE CONSERVERS

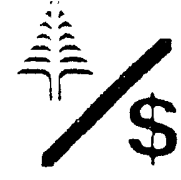

.

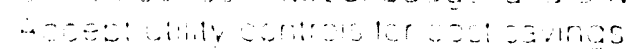

iinter:

HASSLE AVOIDERS

- Minimize hassle in buying appilances

- Wani personal conirol

- Worry ine leasi about cosi or saiely

\section{VALUE SEEKERS}

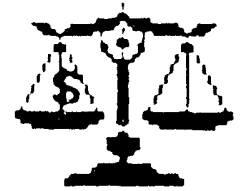

- irivesi trme un duvirg aspliances fomoarison SnODi

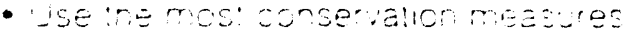

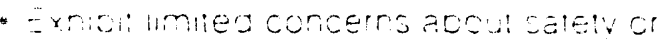
asoserance

Residential Market Segments: Percentage of U.S. Customers
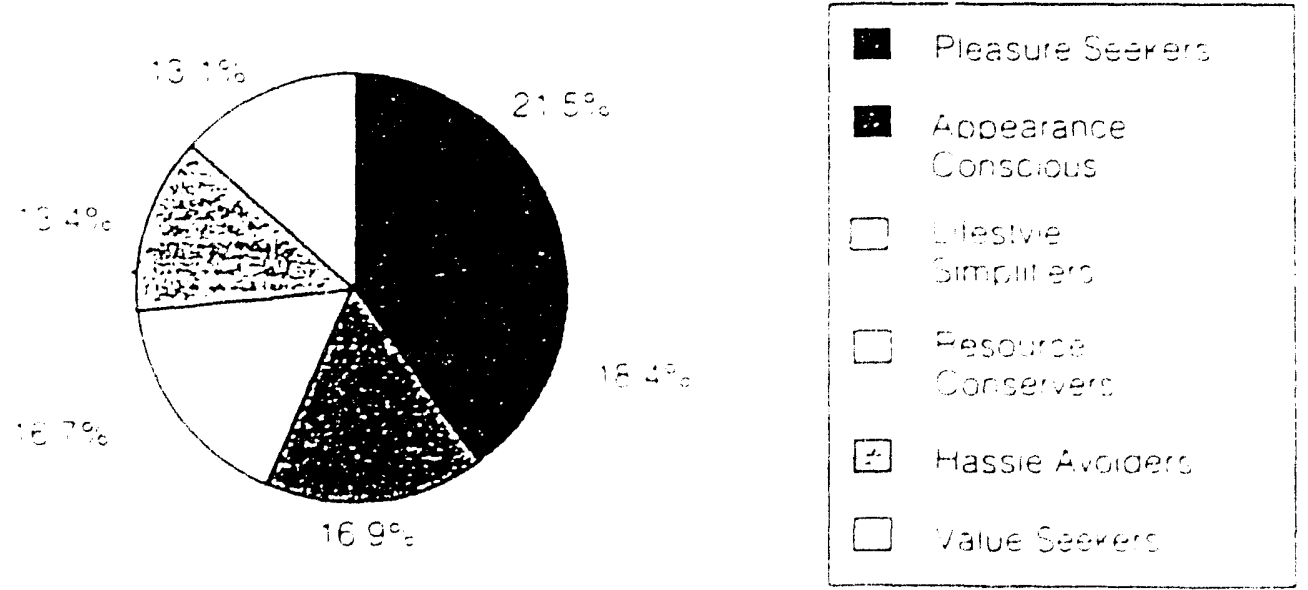
Attachment C. to Issue Paper No. 7

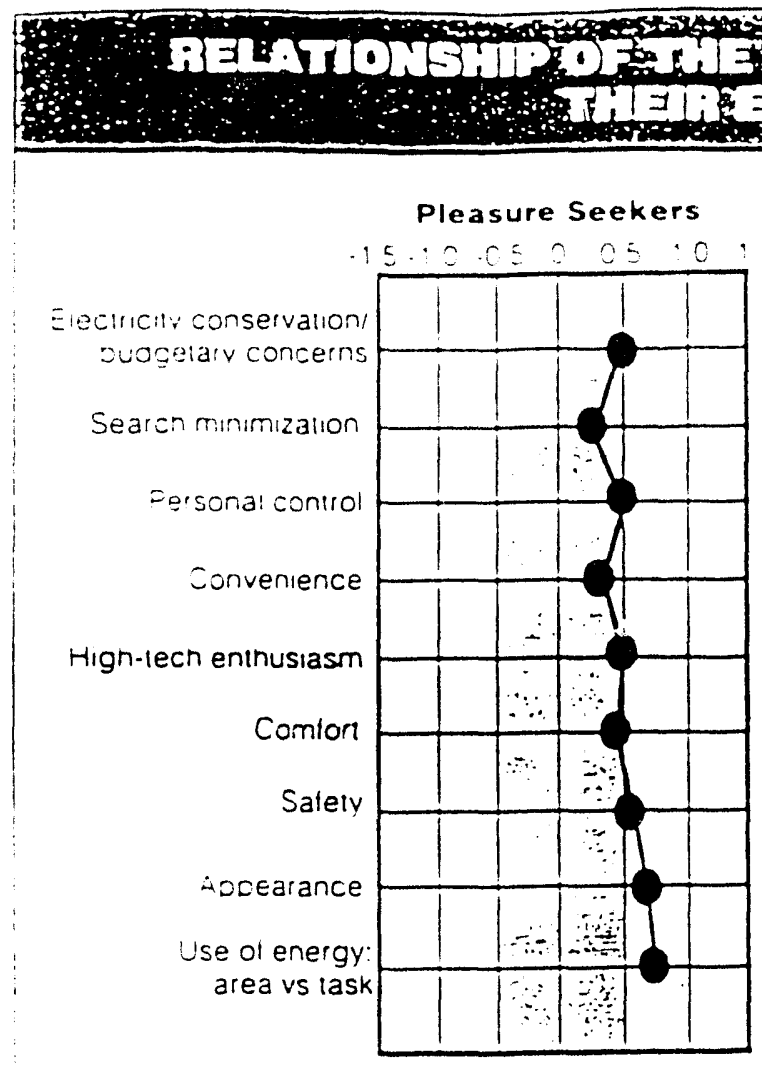

Appearance Conscious

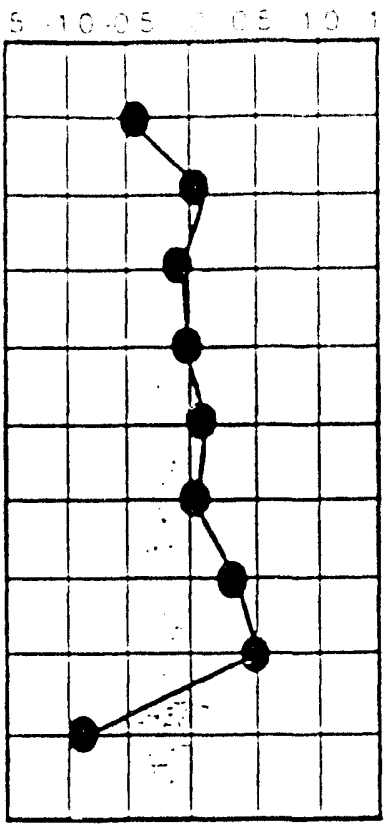

Lifestyle Simplifiers

$15.10-050051015$

Electricliv conservation

oucgetary concerns

Searcn minımizalion

Personal control

Convenience

-ign-lecn eninusiasm

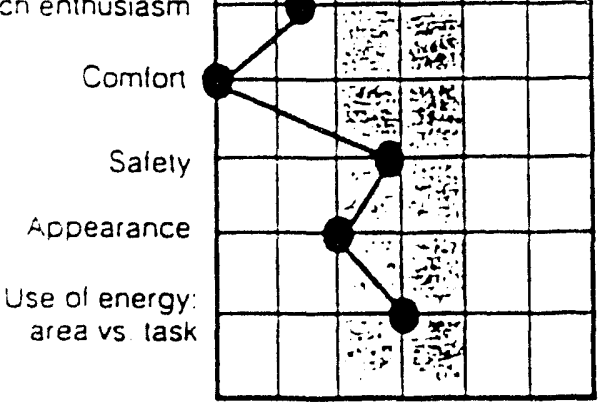

Hassle Avoiders

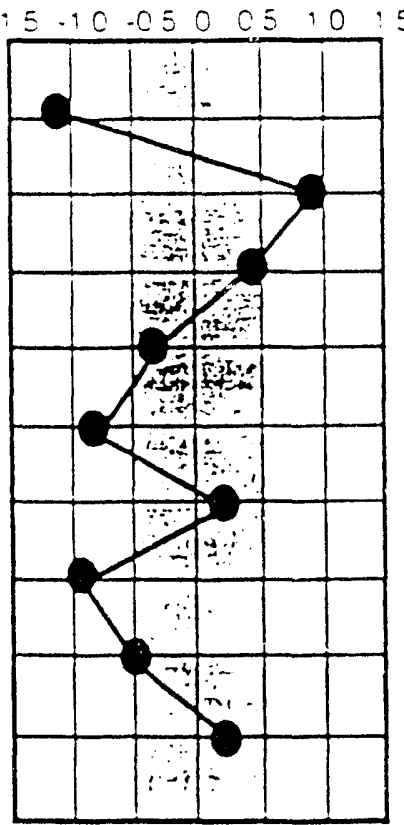

Resource Conservers

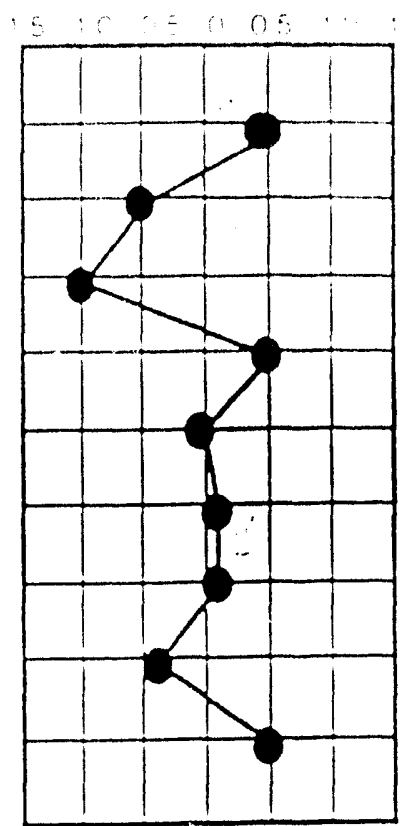

Value Seekers

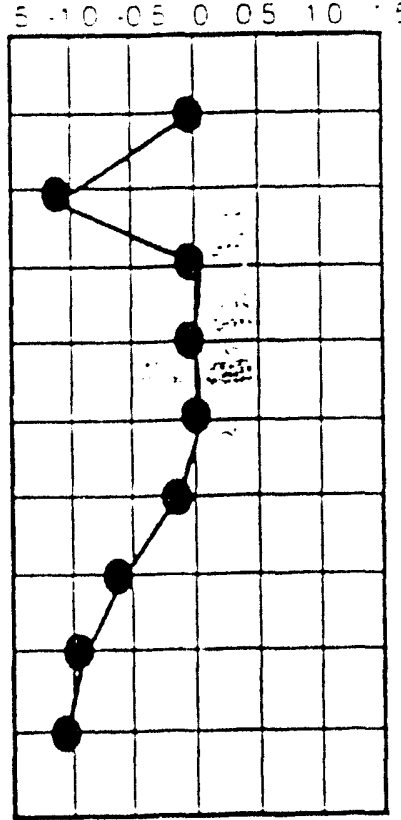

The numbers on each grid are slandardized lacior scores .. Iney indicate how posilively or negalıvely a segment leels aboul each needs/benefils tacior relalive to the other segrnents 
Attachment D to Issue Paper No. 7

Table 1

RESIDENTIAL ENERGY USE IN THE UNITED STATES IN 1987

\begin{tabular}{lccc}
\hline Quadrillion BTU) & To:al & Fossil Fuel & Electricity \\
\hline Space Heat & 6.15 & 5.86 & 0.35 \\
Water Heat & 1.86 & 1.42 & 0.43 \\
Cooking & 0.42 & 0.23 & 0.18 \\
Appliances, Light, & 1.96 & 0.00 & 1.96 \\
A/C & & & \\
\hline
\end{tabular}

$\begin{array}{llll} & \text { TOTAL } & 10.39 & 7.46\end{array}$

Source: Estimated from Residential Energy Consumption Survey (RECS)

(1989). Energy Information Administration, U.S. Dept. of Energy.

Note: Dala are for $1986 / 7$.

Table 2

CONCEPTUAL FRAMEWORK

MOTIVATION TO ACT: Needs, Wants, Values, Emotions

FACTORS THAT ENABLE Socio-economic \& Environmental

OR CONSTRAIN ACTION: Factors:

Demographics

Physical Infrastructure

Personal

Societal

Social/Legal Factors

MANISFESTATION OF Behavior/Activities

CONSTRAINED OR

ENCOURAGED MOTIVATIONS:

MEASUREMENTS:

Frequency, Duration, Sequence

Time

Distance

Location \& Type of Activity

Expenditure Patterns:

Recent and Historical 
Issue Paper No.: $\quad x$

Name of TAC: HERS

Contact person: Doug Swart, City of Fort Collins Light and Power

Date of issue paper: October 24,1991

Title of issue: Do HERS have to differentiate hetween new and existing housing'? If so, how'?

Recommendations (if any) (include majority/minority): New and existing homes should not he differentiated, from a technical standpoint, in national HERS guidelines.

Summary of analyses already completed: N/A

Alternative solutions with advantages and disadvantages:

Alternatives:

1. Differentiate.

- Many existing HERS and certification programs target either new or existing homes only, due to program sponsor goals in the marketplace.

- Players, timing, and other aspects affecting program delivery differ in the two marker segments.

- Some feel utility hilling histories can be used to establish a rating for existing homes. There is little consensus on this idea.

- Retrofit analysis is unique to existing homes (see HERS Issue Paper No. 10). However the same techniques may he applicable to improving new home design.

- Data collection technicues differ. New homes can be rated from plans and specifications (with site visits to confirm data). Existing homes may take longer to rate, and the rater must play a detective role when assessing energy features. Default values must he used on occasion when access is not possible.

2. Do not differentiatc.

- From a technical standpoint, heat transfer rules apply equally to new or existing homes. There is no inherent reason why a rating cannot be calculated for both categories using the same methodology.

- One HERS can he developed with multiple applications. Delivery aspects of a unified program can be customized for new and existing homes.

- Rating both sectors using the same approach, on a common scale, gives users an accurate picture of the full range of energy efficiency in the housing stock. It enables direct, "apples-lo-apples" comparisons 10 he made hetween new and existing lomes.

- A new home hecomes an existing home the day it is occupied. Rating hoth market segments with a single program means that a new home rating remains applicable when the home is sold. A new rating would tiot he required unless physical changes are made. 
Due to the data collection challenges, there may be somewhat less confidence in ratings for selected existing homes. In most cases this will not he a problem. In any case, a rating with some uncertainty will almost always he more useful than no rating at all. Apart from data collection, implementation aspects are likely more critical than technical aspects as local programs decide whether or not (1) differentiate new and existing homes.

Research still needed (if any): N/A 
Issue Paper No.: $\quad$ ()

Name of 'TAC: HERS

Contact person: Bill Prindle, Alliance to Save Encrgy

Date of issue paper: Oclober $3(1,1991$

'Title of issue: $\quad$ To what extent should there he linkage hetween HERS and EEMs?'

Recommendations (if any) (include majority/minority):

HERS should produce the reliable energy information needed to process EEMs:

- To qualify for an EEM an energy rating in conformance with these guidelines must he performed.

- If the home is heing rated for an EEM program, the HERS must produce an accurate technical rating that places the home on a $1(x)$-point scale. If required hy the EEM program, it should also produce an aciurate annual energy cost projection for the home.

- If the home is to he retrofitted, the HERS must accurately estimate the energy and dollar savings projected from a specific package of proposed relrofits.

Summary of analyses already completed: This analysis is hased on the HERS Technical Advisory Committee's (TAC's) current understanding of how EEMs work, and on the experience of HERS operating in the field.

\section{Alternative solutions with advantages and disadvantages:}

1. Require no uniform HERS outputs for EEMs-This would make it easier for current HERS programs to claim compatihility with EEM programs. It would not, however, help create the consistency and reliahility for which many Collahorative Consensus Committe memhers have expressed an urgent need.

2. Eliminate the uniform rating scale and report basic energy use and cost numbers only. This presumes that EEM programs would eliminate the ratio stretch underwriting approach and go to a PITI+E or similar approach, which eliminates the need for the scale-and-threshold basis for the ratio stretch approach. While this might satisfy some lenders by hasis underwriting on harder numbers, it takes away the consumer information benefit of knowing how the home compares to others. It also requires more work for underwriters to plug in specific numbers for each home rather than making a simple ratio stretch hased on fixed criteria.

Research still needed (if any): The HERS TAC needs to monitor the progress of the EEMs TAC; the specifics of our recommendations on this issue depend on the specifics of their EEM recommendations. This is particularly true with regard to the ratio stretch vs. PITI+E underwriting issue, and the economic analyses required hy the emerging EEM procedures.

NOTE: While the HERS tool may not he the hest or only means (o achieve this, the EEM process will also require economic analysis suffi:ient $t$ detertiine whether the package of retrofits is cost-eflective, produces positive cash flow, and meets appraisal criteria related o present value of savings. 
Issue Paper No.: $\quad 10$

Name of TAC: $\quad$ HERS

Contact person: $\quad$ Bill Prindle, Alliance to Save Energy

Date of issue paper: Octuher 2, 1991

Title of issue: $\quad$ What information should HERS guidelines require vis a vis retrofits? Will HERS provide dollar cost and savings by component of proposed retrofit?

\section{Recommendations (if any) (include majority/minority):}

- HERS calculation procedures should accurately project energy savings in energy units and dollars for proposed retrofits.

- A uniform procedure and set of assumptions should be developed for economic and financial analysis of retrofit measures, especially if the retrofits are to be financed through an EEM. Energy savings calculations from HERS tools would become an input to such a procedure.

Summary of analyses already completed: This analysis is based on the HERS Technical Advisory committee's (TAC's) current understanding of how EEMs work and on the experience of a number of working HERS programs.

\section{Alternative solutions with advantages and disadvantages:}

1. Set no requirements for HERS analysis of retrofits. This would leave local programs free to operate as they desire, but would not give lenders added confidence in the accuracy of estimated savings from energy retrofits. The Collaborative Consensus Committee (CCC) seems to want to increase confidence in the reliability of HERS, as one of its basic tenets.

2. Set no economic analysis guidelines for HERS and leave all economic calculations to lenders. This 'would make HERS programs' lives easier but lenders' lives harder. CCC sentiment seems to support making lenders' lives easier to induce them to use EEMs more frequently.

Research still needed (if any): The EEM TAC needs to give the HERS TAC more guidance on what EEM practitioners need in the way of data from HERS. This is especially true in the area of economic data. In this area, research is needed on the most appropriate cost-effectiveness test to apply and on the method for assigning appraised value to energy retrofits on the basis of capitalized energy cost savings.

NOTE: The mortgage industry and the EEM TAC should develop specifics for the uniform procedure recommended above. The procedure should include basic cost-effectiveness tests, cash-flow analyses, and information sufficient to complete the appraisal calculations in FHLMC Form 70-A or its successor. 
Issue Paper No.: 11

Name of TAC: $\quad$ HERS

Contact person: William Freeborne, U.S. Department of Housing and Urhan Development

Date of issue paper: October 7, 1991

Title of issue: $\quad$ Are ratings required for each building, or can group ratings be fermitted? (or subsets of ratings)?

Recommendations (if any) (include majority/minority): Building ratings should be grouped logether where possible and where quality control can be demonstrated. The grouping will have the henefit of reducing duplication of effort as well as providing a common hasis for the comparison of homes (i.c., buyers will know that a group of homes have similar ratings for comparison with another different group of homes). The group rating shall be appropriate for the same model huilt multiple times.

Summary of analyses already completed: American Society of Heating, Refrigerating, and AirConditioning Engineers (ASHRAE) Special Project 53 Position Paper \#2-2 determined that two prototypes (single-family detached and single-family attached) could characterize housing for the Automated Residential Energy Standard (ARES). Energy savings are linear with size (square foot, linear foot, or air change basis).

Research still needed (if any): A sensitivity analysis needs to be made similar to the ASHRAE Special Project 53 to determine if HERS can be applied to prototypes without losing needed accuracy and to determine baseline characteristics.

Apartment buildings should be looked at to determine if each unit should be rated individually or should the building be rated as a whole? A sensitivity analysis would be done to determine the effect of solar orientation or common walls on units plus whether the units are individually metered or whether there is central mechanical equipment. 
Issue Paper No.: 12

Name of TAC: $\quad$ HERS

Contact person: Stephen S. Szoke, Passive Solar Industrics Council

Date of issue paper: September 9, 1991

Title of issue: What building types should be included in HERS?

Recommendations (if any) (include majority/minority): HERS should be applicable to multifamily, single-family, and manufactured housing.

Alternative solutions with advantages and disadvantages: Limit HERS to structures that are easy to model, analyze, and evaluate such as single-family detached and possibly townhomes. HERS remains simple, but energy-efficient mortgages may not be fully applied and may not be considered where they would be most valuable, i.e., multifamily structures.

Research still needed (if any): Appropriate methods of modeling common walls and floors/ceilings and considering one mechanical system serving more than one dwelling unit should be considered. These considerations must be addressed in the modeling tool and there needs to be a way to economically incorporate the effects into any simplified HERS.

Background: Since the home energy rating system (HERS) is being developed to be used as a tool for energy-efficient mortgages (EEMs), it is imperative that the rating system be most applicable to the lowest cost housing. This is important to have more people qualify for housing based on good energy-efficient designs when utility bills are incorporated into the considerations for obtaining a mortgage. The application of EEMs to single-family detached structures could be pursued to improve the level of energy efficiency for single-family structures, but would probably have only a minor impact on the number of individuals who could qualify for the mortgage, particularly since increases in energy efficiency increase the cost of the structure.

In multifamily structures, the increased ccsts for energy efficiency tend to be less per family because of reduced exterior envelope for the same square footage. In addition, individuals considering the purchase of units in multifamily complexes tend to be in greater need of energy efficiency considerations when qualifying for a mortgage, because they tend to have lower incomes.

Townhomes, duplexes, multiplexes, and condominiums need to be incorporated into all home energy rating systems on an equal basis as single-family detached structures. Difficulties that must be overcome include how the analysis and evaluation techniques consider common walls and how to analyze and evaluate mechanical systems serving more than one living unit. 
Issue Paper No.: 13

Name of TAC: $\quad$ HERS

$\begin{array}{ll}\text { Contact person: } & \text { B. D. Howard, Alliance to Save Energy }\end{array}$

Date of issue paper: Octoher 4, 1991

Title of issue: $\quad$ What features should be included in a HERS?

Recommendations (if any) (include majority/minority): To produce an acceptable annual energy consumption estimate suitable for deriving a rating in Btu/year (see HERS Issue Paper No. 3), and other relevant energy ratings data, the following key building subsystems should be analyzed:

- Thermal envelope

- Mechanical system

- Hot water service

- Other loads (hard-wired lighting and appliances).

Summary of analyses already completed: A review of how these features are handled in existing HERS programs was conducted by the committee, and discussions of what features were most important were undertaken. A paper by Rosenfeld and Wagner (Lawrence Berkeley Laboratory) from the 1982 ACEEE "What Works" Conference, was reviewed for preliminary guidance on the issue. An issue brief from the Alliance to Save Energy was also reviewed.

Alternative solutions with advantages and disadvantages: To develop an annual energy utilization estimate allowing comparison of (a) competing options for increasing energy efficiency in design (new homes) and (b) comparison of homes on a level playing field, such as new versus existing homes, a basic minimum set of features needs to be addressed. The recommended list gives such a baseline, while excluding other features that may be more sensitive to occupant-based variance. Sonderegger (1978) and Wilson et al. (1982) found evidence that annual energy use can be influenced by the use of small appliances, and variances of 2:1 exist between the households with highest and lowest energy consumptions in structurally similar buildings.

Research still needed (if any): It would be useful to know the actual energy savings potential of energy conservation measures beyond the scope of the primary list, such as home automation and zoning and high-tech control systems, natural architectural cooling design, and distribution (duct and piping) efficiency. As these data become available and greater conidence is gained in estimating their role in energy ratings, additional features may be added to the key list. Provision will need to be made for calculationally difficult and innovative features as they appear.

\section{Rs:ierences:}

Rosenfeld, A. H. and B. S. Wagner. 1982. "Technical Issues for Building Energy Ratings." ACEEE Conference, Santa Cruz, California (pp. 338-402).

Sonderegger, R. C. 1978. "Movers and Stayers - The Resident's Contrihution of Variation Across Houses in Energy Consumption for Heating." (In: Socolow, Saving Energy in the Homle, 1978. Princeton U. Press. 
Wilson, N. W. et al. 1982. "Occupant Effects on Residential Energy Consumption." ENERGEX Conference Canada (pp. 57-70). 


\section{Attachment to Issue Paper No. 13 \\ Outline of Key Technical Features to be Considered}

Building Envelope:

Thermal protection (insulation, heat capacity)

Windows (U-value, shading coefficient, air leakage)

Doors (U-value, air leakage)

Foundation system (U- or C-value, insulation position, heat-cap)

Air infiltration (using blower-door, ASHRAE Std. 119)

Passive solar performance

Thermal mass

Architectural shading

Mechanical Systems: (heating, cooling, ventilation)

Parameter: Equipment seasonal efficiency ratings (AFUE, HSPF, SEER)

Minimum system types: (where climatically applicable)

Furnace (nat. gas, fuel-oil, electric)

Heat pumps

Boilers (nat. gas, fuel-oil, electric)

Baseboard electric system

Air-conditioning (electric and nat. gas)

Active solar space heating

Mechanical ventilation (if fitted):

Hourly ventilation rate

Ventilation effectiveness (estimated)

Estimate of: Distribution system's effectiveness

Hot Water Service:

Tank effectiveness (conventional fuels)

Tankless heaters

Instantaneous water heaters

Heat-pump water heaters

Solar systems (active and passive)

Piping insulation (optional check-off, bonus pts.)

De-super heaters

Optional Features (for bonus points, or hourly simulation/trade-off purposes)

Other loads: (hard-wired electric lights and major appliances)

Controls:

Set-back thermostat(s)

DDC systems (SmartHouse, CEBus, etc.)

Zoned control systems 


\section{Preferential Utility Rate Schedule(s)}

HVAC integrated thermal energy storage systems

(Option A) Time of use pricing

Photovoltaics 
Issue Paper No.: : it

Name of TAC: $\quad$ HERS

Contact person: Doug Swark, City of Forl Collins Light and Power

Michat Holte. Architectural Energy Corporation

Date of issue paper: October 4.1991

Title of issue: What level of detail/accuracy is necessary"?

Recommendations (if any) (include majority/minority):

1. In order 10 be certified as an "approved HERS 1001, , absolute calculational accuracy within a prescribed energy error hand (in Btu/yr) relative to the henchmark certification results must he demonstrated. The error band should recognize the trade-off hetween accuracy and the cost of performing a rating.

2. No specific level of detail of HERS tool models and inputs should he prescrihed-any tool that passes the certification tests may be used, regardless of level of detail.

3. Provide rater training and quality assurance measures to minimize input errors.

Summary of analyses already completed: Research hy Pacific Northwest Laboratory and Lawrence Berkeley Laboratory; Colorado HERS preliminary simplified inputs analysis by Architectural Energy Corporation; Pennsylvania Home Energy Cost Estimator work hy Equinox, Inc.

Alternative solutions with advantages and disadvantages: HERS tools' accuracy specifications should he hased on lender requirements, halanced against state of the art in potential HERS tools and realities of the rating process. Approaches to selling accuracy targets are discussed in the attachment. It is tempting to set a very stringent criterion. However, very few tools will then he certified, the level of detail of inputs required to get the "right" answer may be so great that the tool is impractical to use, and the accuracy of results may he limited hy the accuracy of key input data (such as air leakage rate). A "good" estimate for energy cost may not he a "perfect" estimate, hut it will be far hetter than the information now typically available to lenders.

Energy calculations may he done at any level of detail, from very simplistic to exceedingly rigorous. From a HERS perspective, there are trade-offs at every level hetween accuracy and practical, affordable implementation (see attachment). Four key aspects can limit the accuracy of energy calculations: (1) model detail, (2) model accuracy, (3) input detail, and (4) input accuracy. In the past, models were typically chosen to make calculations amenable for manual methods or early microcomputers. With faster microcomputers and sophisticated modeling software hecoming widely available, there is little reason not to choose relatively detailed and accurate models-accuracy of results should not he limited hy aspects (1) or (2). Choices on aspect (3) are less straight forward for HERS applications. Highly detailed inputs have the hest potential to produce accurate results, hut involve more data collection and preparation time (cost) and potential for error as well. Simplified input approaches (see attachment) are a promising approach that could be pursued. Aspect (4) relates to rater training and quality control. 


\section{Attachment A to Issue Paper No. 14}

Energy calculation ontions: The accompanying figure shows a range of energy calculation methods that could be used for a HERS tool, ranging from little detail to a high level of detail. In the continuum shown, detail can vary in two ways: the model and/or the inputs (these two aspects don't always go handin-hand). Also shown are other characteristics that may vary accordingly. In general, the most detailed approaches have the potential to produce the most accurate result. However, models requiring detailed inputs typically require highly trained users, more time, and higher cost. They also provide greater opportunity for making errors (hundreds of inputs, temptation to take shortcuts, etc.). Result accuracy may he limited, too, by the accuracy of particular key input data (such as air leakage rate). Therefore, it is questionable whether the potential accuracy of a detailed approach is achieved in the real world.

Model detail: In the past, models were chosen on the basis that calculations be amenable for manual procedures and early microcomputers. As more powerful microcomputers hecome widely available, it makes sense to use relatively sophisticated models. The appropriate level of model detail depends on the accuracy deemed necessary by end users, and can be judged using HERS certification methods.

Input detail: ${ }^{2}$ The appropriate level of detail of the inputs is less straightforward and could vary depending upon the application. Homes with simple geometry and uniform energy features (e.g., the same $\mathrm{R}$-value used in all exterior walls) can be accurately modeled with relatively few inputs. In contrast, homes with more complex geometry or features (e.g., buffer spaces, vaulted ceilings, active solar systems, sunspaces, thermal storage walls, partially finished basements, additions/remodels, etc.) may require detailed inputs to produce an accurate description.

Simplified inputs: Most HERS in the past have chosen one level of detail and used it for all homes, paying the price of being overly detailed in some instances and inaccurate in others. Another approach is to have a tiered system in which two or more levels of input detail are available. To provide consistent results, two different front ends could provide data to the same model. When the simplified set of inputs is used, the software would incorporate built-in assumptions to translate these inputs into the format required by the model. When the detailed set of inputs is used, the assumptions are hypassed.

A home would be screened initially to determine which level must be used to achieve the accuracy target. Most homes could likely be rated with the simpler (and presumably less costly) approach, with the remainder requiring the more detailed method(s).

Research is needed to evaluate the classes of homes that can he appropriately handled using detailed vs. simplified inputs. The starting point can he data for homes that have already heen rated using a detailed approach. For example, in Fort Collins, Colorado, more than 30() homes have already been rated in detail-they can be re-rated using fewer inputs and more assumptions, and results compared. Similar data is likely available from other existing HERS. Architectural Energy Corporation completed a feasihility study of simplified inputs using a small subset of the Fort Collins data. Results were promising, showing the likely feasibility of such an approach. More work is needed to identify alternative approaches and clarify the range of applicability. It is important that these tests he performed on real buildings--which are almost always more complex than those imagined hy researchers! Companion research is also needed to determine the time and cost savings that a simplified inputs approach can produce. Further, implications of this method on the choice of a rating scale of appropriate resolution should also be investigated.

"It's not relevant to "minimum" standards for HERS. It is up to individual HERS to find ways to he cost effective. 


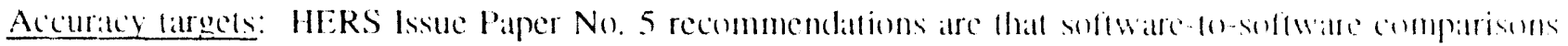

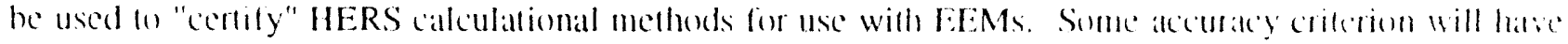

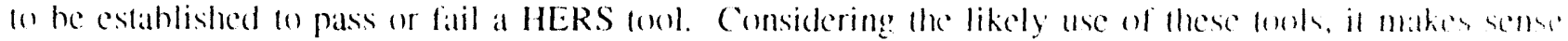
we reference the standards to energy cost error, on an ahsolute hasis. for example. londers maty say they need energy dollar cost estimates within \$15 per month of "truth" (i.e.e results af detitited eneres

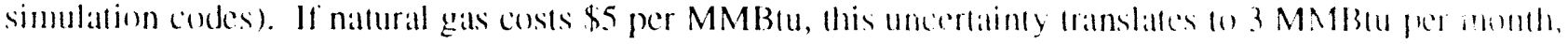
or $18 \mathrm{MMB}$ M/yr. This same error hand should be used, on an ahsolute hasis, for all henchmark test calses,

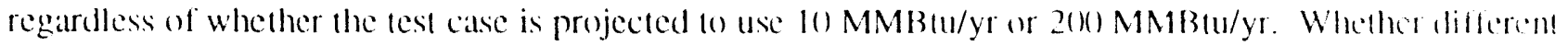
error ranges should be used for different energy sources is open to dehalle. 
Attachment B to Issue Paper No. 14

\section{DETAIL/ACCURACY CONTINUUM}

MODEL

DETAIL

WEATHER

DATA

INPUT

DATA

Detailed simulation

"Simplified" methods
(VBDD, Heat Balance, Correlations)

Prescriptive checklist

$$
\text { Hourly }
$$

APPLICABILITY

\section{Detafled \\ geometry spectfied}

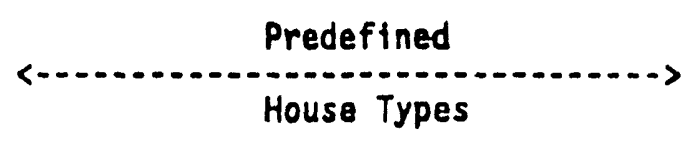

Energy

features

may vary

Buffer spaces

explicitly accounted for
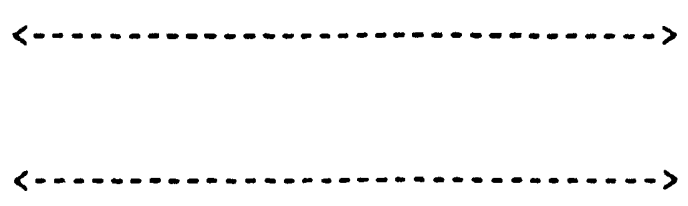

Any home

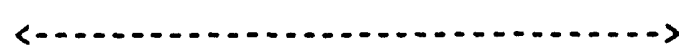

Few

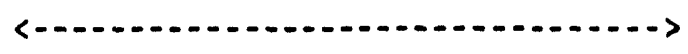

NUMBER OF

ASSUMPTIONS

TIME/COST TO COLLECT \& PREPARE DATA

PROBABILITY OF RATER ERRORS

POTENTIAL

ACCURACY
High

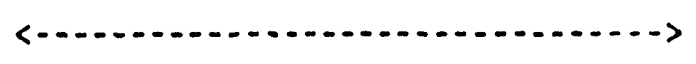

High

High

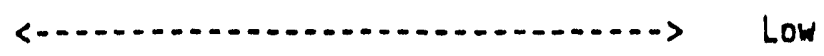

Low

Low

No

geomerry spectfied

Energy feature uniform

Buffer space ignored

Frame home of simple geometry and uniform

features

Many

Low

NOTE: There is not, in all cases, a direct correlation among the various ranges shown. 
Issue Paper No.: 15

Name of TAC: $\quad$ HERS

Contact person: $\quad$ Ron Judkoff, National Renewable Energy Lahoratory

Date of issue paper: Octoher 1,1991

Title of issue: $\quad$ How do we include innovative huildings/features in the HERS, yet keep it from heing too cumbersome?

\section{Recommendations (if any) (include majority/minority):}

1. Allow partial qualification according to the climate zones for which the local HERS is intended.

2. Allow for an alternative rating tool where the usual rating tool is too simplified to handle innovative huildings, energy features, or new technologies.

Summary of analyses already completed: The software qualification test cases discussed in HERS Issue Paper No. 5 can be defined to test a HERS calculation method for any building type or feature that can be modeled with state-of-the-art simulation programs. To keep this from becoming too cumbersome, a particular HERS calculation method would not have to pass all test cases, but only those cases representing the building types/features on which it was intended for use. For example, a HERS intended for use in Alaska would not have to pass test cases on cooling equipment, or a HERS intended for use in Miami would not have to pass test cases on high efficiency heating features. HERS calculation methods intended for a wide variety of climate zones, building types, and energy features would have to pass a greater number of qualification tests than those intended for a narrow range of applications.

Another approach would be to require that all local HERS be able to provide an alternative rating tool when the normal tool is too simplified to handle the building in question.

\section{Research still needed (if any):}

1. Research would be necessary to develop algorithms to upgrade the alternative rating tool for new technologies.

2. Research would be necessary to develop simplified algorithms or correlations to incorporate new technologies in the normal HERS calculation methods. 
Issue Paper No.: 10

Name of TAC: HERS

Contact person: $\quad$ Ron Hughes, HERS Industry (Energy Rated Homes of America)

Date of issue paper: Octoher 4,1991

Title of issue: What are the minimum required outputs of HERS tools?

Recommendations (if any) (include majority/minority): HERS tools will uniformly include at least the following items:

$\underline{\text { Record keeping }}$

Name of the rating system

Name of analysis tool

Rating scrial number

Owner and address

Phone

Builder and Address

Certified raters name

Date of rating

Climate and/or weather file

Utility name(s)

\section{Thermal efficiency descriptors}

House type

Floor area

Efficiency Level of:

Ceiling

Wall

Floor

Windows

Air leakage

Thermal mass

Roof overhang (shading)

Glass area cach direction

Estimated solar contribution (active, passive, etc.)
Space heating and cooling equipment Manufacturers efficiency rating Adjusted efficiency (location, insulation, and air leakage of ducts and plenum.

Zoning

Setback thermostats

Water Heater

Location

Efficiency

Fuel source

Type (conventional, demand, solar, etc.) Heat recovery on a.c. and/or other devices

\section{Rating and Costs}

Total point or rating score

Efficiency rating on 0-100 point scale

Estimated purchased energy

Space heating

Space cooling

Water heating

Lights and appliances

$\mathrm{Btu} / \mathrm{sq} \mathrm{ft} / \mathrm{yr}$

Total MMBtu per yeas

Cost of energy per MBtu per utility

Total estimated annual energy cost

How the house compares to local energy code

Alternative solutions with advantages and disadvantages: Most scaled HERS tools are generating these outputs already. Consideration of this data is necessary for an accurate and comprehensive estimate of annual energy consumption, but it goes beyond the scope of many certification and prescriptive programs.

Alternative 1. Only Summary Shect: Name, address, rating, estimated annual cost, haseline (average) energy costs, and improvement recommendations.

Advantages/disadvantages: This is the most important information for lenders. Leaves many questions unanswered for home owner or home huyer. 
A.lternative 2. How the house compares to local energy code.

Advantages/disadvantages: Most useful for new houses if the rating is tied to code (as in Florida) or a code alternative (as in Alaska). Codes are minimums. Requirements for a rating of "efficient" are almost always higher than current minimum codes.

Research still needed (if any): None. 
Issue Paper No.: 17

Name of TAC: $\quad$ HERS

Contact person: $\quad$ Ron Nickson, National Association of Home Builders

Date of issue paper: Octoher 9, 1991

Title of issue: $\quad$ Should HERS have a code link?

Recommendations (if any) (include majority/minority): HERS does not need a tie to codes; however, it may have a tide to codes (see discussion).

Summary of analyses already completed: Although a HERS program and energy code will of necessity address many of the same issues, the goals and the hasis of the two programs differ. These include:

- Requirements: HERS type programs rate houses (most likely new and existing) for energy efficiency, while code provisions are designed to establish the minimum requirements for new construction.

Many states and local jurisdictions have written energy code provisions that are designed around energy use and requirements that respond to the needs of the local governing jurisdiction or state. Even though many of these provisions are approaching the maximum or near maximum levels of energy efficiency for certain components of the building, they do not reflect the necds of a HERS program, do not address all of the requirements of a rating program, and do not provide for ratings and different levels of performance.

- Legal Responsibility: Codes when adopted are legal requirements for the community and enforced through the actions of a governmental agency. HERS programs should be voluntary and encourage participation by creating incentives for better energy conservation. 
Issue Paper No.: 18

Name of TAC: HERS

Contact person: $\quad$ Steve Baden, National Association of State Energy Officials

Date of issue paper: Octoher 21,1991

Title of issue: $\quad$ Under what conditions is re-rating required!?

Recommendations (if any) (include majority/minority): From a technical viewpoint, a home energy aating is good for as long as the home's energy features do not change.

\section{Alternative solutions with advantages and disadvantages:}

1. Set time linit.

Advantage: It would be clear to lending institutions that information regarding a home fits within a time span.

Disadvantages: Time limil would be artificial. The home could he changed within time period, and the home also may still have the same characteristics after the period. It would require, in some cases, unnecessary expense of re-rating when not really required.

2. Re-rated at point of sale.

Advantage: It would he the most secure for the home buyer and financier.

Disadvantage: It would require, in many cases, the unnecessary expense of re-rating when not really required.

Research still needed (if any): Research on time variations in the persistence of energy saving measures is needed.

Background: The issue was studied hy the housing industry technical advisory committee in the development of a home energy rating system in the State of Alaska. The committee unanimously accepted the above recommendation. In marketing of the Alaska program, consumers are advised to "know hefore they huy" and ask for a new rating of the home. The program does not require that a re-rating must take place unless the home has been modified since the last rating.

Homes for which energy-efficient mortgage financing is desired should he rated at the time of sale or time of refinancing unless:

- A rating was done previously and the energy fealures of the home have not changed

- The rating scaale has not changed. 
Issue Paper No.:

Name of TAC: $\quad$ HERS

Contact person: $\quad$ Bill Prindle, Alliance to Save Energy ${ }^{3}$

Date of issue paper: Octoher 30, 1991

Title of issue: $\quad$ Should there he a national threshold for qualifying for an EEM? If not, how will local thresholds be developed?

Recommendations (if any) (include majority/minority): There should be a consistent national basis for qualifying for an EEM.

1. If a lixed ratio stretch basis is used, these should be a consistent national threshold of energy efficiency applied to 10()-point scales determined at the state or local levels.

2. If the basis is keyed to energy savings of the individual home in relation to a reference home (through PITI-ES or a similar approach), then there need be no fixed threshold on the 10()-point HERS scale.

Summary of analyses already completed: This analysis is based on the two major EEM underwriting approaches currently being considered hy the Collaborative Consensus Committee.

\section{Alternative solutions with advantages and disadvantages:}

\section{Alternative solutions}

1. If the fixed ratio approach is used, the underwriter's job is simpler in that each energy-efficient home is treated the same. Once a given design is approved, lenders in the area can casily process EEM loans on them. However, it may create distortions if the fixed ratio stretch does not capture the real financial henefits of the home's energy savings; e.g., if a $2 \%$ ratio stretch imputes $\$ 600$ in energy savings, but the home saves $\$ 300$, the EEM is over-crediting energy efficiency. A solution would be to set local thresholds on the HERS scales so that, based on local data, the threshold comes close on average to justifying the ratio stretch for that area.

2. If the energy-savings-based underwriting is used, the underwriter is more assured that the individual loan more closely reflects the real energy efficiency of the individual home. But the underwriter has to obtain energy savings data for each home and apply it in each underwriting. The key is to provide, through the HERS guidelines or other sources, reliable and regionally accurate data on the home being underwritten, the average housing stock, and perhaps some reference homes (e.g., code-built homes, mean and median homes, means by size and typed, etc.) If underwriters can he provided this data, this approach could work.

Research still needed (if any): If the ratio stretch method is used, research is needed on the relationship hetween stretched ratios and actual energy savings for various efficient home designs, compared to reference homes, in different regions. This should lead to research on setting a threshold(s) on the 100)-point HERS scale, either nationally or regionally, so that ratio stretches used by underwriters are reasonably close representations of the actual energy savings produced by a given home.

${ }^{3}$ Now with Barakat and Chamberlin, Washington, DC. 
Issue Paper No.: 1

Name of TAC: $\quad$ EEMs

Contact person: Bill Brewster, Mortgage Bankers Association

Date of issue paper: October 8, 1991

Title of issue: What data or analyses do we have that energy-efficient mortgages increase housing affordability? Do any field data or analyses demonstrate how the homeowner benefits? How many more households might qualify for housing? What analyses have been done (or need to he done) on the impact of energyefficient mortgages on portfolio risk?

Recommendations (if any) (include majority/minority): The TAC recommends that the appropriate administering state, regional, and federal agencies label as "EEMs" those loans that include the consideration of reduced energy costs in the mortgage underwriting process. This would enable their default/delinquency performance to he monitored.

Summary of analyses already completed: Many studies illustrate that low-cost financing of energy improvements can result in a net savings to home buyers and homeowners. An EEM gives home buyers an opportunity to finance the cost of energy improvements as part of a 30-year first mortgage. This is the least expensive financing avaiiable. The relatively low increase in mortgage cost may then be offset by a reduction in energy cost resulting in a net savings, which increases housing affordability. In 1986, the MIT/Harvard Joint Center for Housing Studies indicated that a national HERs/EEM program could induce an additional 250,000 families a year to become first-time homeowners nationwide.

"'Energy Efficient' Mortgages Can Increase Buy:ng Power," H. Jane Lehman, The Washington Post, October 14, 1989.

"Energy Efficient Mortgages Are Gaining Appeal," Vic Simon, Real Estate Finance Today, July 28, 1989.

Guidelines for a Uniform Energy Efficient Mortgage Program, prepared for the U.S. Department of Energy by the Uniform Energy-Efficient Mortgage Steering Committee, Palo Alto, California, March 1, 1989.

Making Housing More Affordable Through Energy Efficiency, The Alliance to Save Energy, Washington, DC, June 21, 1988.

The Effect of the Shelter Industry Energy Rated Homes System on Homeownership, Joint Center for Housing Studies of the Massachusetts Institute of Technology and Harvard University, May 1986.

The Energy Factor in the Market Equation, John M. Teutch, Jr., CMB and James R. Faulstich, Mortgage Banking, May 1985.

Research still needed (if any): Although a considerable amount of research has been done, more analysis is needed in order to accurately assess the impact of an EEM on portfolio performance. The data to perform this analysis will be available once EEMs are differentiated from other mortgages. 
Issue Paper No.: $\quad 2$

Name of TAC: $\quad$ EEMs

Contact person: Walter Patterson, Texas Energy Extension Service

Date of issue paper: October 5, 1991

Title of issue: $\quad$ Should there be a uniform EEM?

Recommendations (if any) (include majority/minority): There should be a uniform EEM.

Summary of analyses already completed: The HERS/EEM Collaborative should strive to suggest a uniform EEM for three valid reasons.

First of all, there is national legislation in the form of the National Housing Act of 1990, which requires HUD, Fannie Mae, Freddie Mac, and the Farmers Home Administration to develop a uniform EEM that accommodates affordable energy-efficient improvements (EEIs) in the mortgage process.

Secondly, the EEM should be uniform to make the process more "user friendly" for lenders, realtors, appraisers, etc. A major finding of a California Home Energy Rating Systems, Inc. national survey ${ }^{4}$ was "strong involvement from banking/lending institutions is needed to strengthen the energy-efficient mortgage component of CFiERS." Uniform treatment of the cost and savings benefit of EEIs in the mortgage process is necess :ry to encourage strong participation from lenders, et al. in the shelter industry.

Thirdly, the uniform EEM should be developed to provide effective financing for energy-efficient improvements. Otherwise, consumer motivation for energy efficiency, which should be stimulated by HERS and energy labeling, will remain unfulfilled because of the absence of effective financing for EEIs.

Alternative solutions with advantages and disadvantages: None.

Research still needed (if any): None.

${ }^{4}$ Public Affairs Subcommittee Report on Legislative Status and Policy Issues; May 31, 1991. 


\section{Issue Paper No.: $\quad 3$}

Name of TAC: $\quad$ EEMs

Contact person: Walter Patterson, Texas Energy Extension Service

Date of issue paper: October 5, 1991

Title of issue: $\quad$ What should be the characteristics of a uniform EEM?

Recommendations (if any) (include majority/minority): The uniform EEM should have the following major characteristics:

1. Treat uniformly the borrower credit worthiness issue of mortgage underwriting by allowing the energy savings of efficient homes (either newly constructed or improved existing) to be effectively recognized as an offset to the additional debt service $(\triangle \mathrm{PITI})$ of energy-efficient improvements (EEIs).

2. Treat uniformly the valuation issue of mortgage underwriting by allowing the cost of supplemental EEIs above baseline requirements to be included in the mortgage at the lessor of their cost or economic value limit. (EEM Issue 4)

3. Make provision for increased loan limits to accommodate inclusion of the incremental cost of EEIs in the mortgage.

4. Make provision for increased loan-to-value (LTV) Ratios to accommodate inclusion of the incremental cost of EEIs in the mortgage.

5. Make provision for supplemental loan assurance to indemnify mortgage guarantors against any added risk or impropriety associated with characteristics number 2,3 , or 4.

6. Integrate with HERS to ensure the work process and information product of the HERS is adequate to support EEM information requirements in scope and accuracy (EEM Issue 10).

\section{Alternative solutions with advantages and disadvantages:}

None. These are the minimum benefits that the EEM should provide if an EEM is to serve as an effective method to finance the marginal cost of EEIs for a broad range of borrowers.

\section{Research still needed (if any):}

1. Identify baseline energy efficiency levels from which energy savings are to be calculated. Suggested alternatives:

- Baseline energy operating cost level to be used in adjustment of huyer credit worthiness could be one of the following:

- HUD scheduled monthly utility cost

- VA scheduled monthly utility cost

- ? percentile home on a 0 to 100 point rating scale. The $25 \%$ home has been suggested as representing the baseline home for which insured lending is available. 
- Council of American Building Officials Model Energy Code (MEC) (if hase deht-to-income ratios are increased hy all mortgage investors/guarantors to accommodate the energy efficiency requirements of the MEC)

- Baseline energy operating cost level to be used in adjustment of an appraisal could be:

- For existing homes-baseline operating cost of the house as is.

- For new construction-baseline operating cost of a house with current minimum construction requirements for the region as required by local codes, HUD MPS, etc.

2. Define "economic value limit" of characteristic \#2.

3. Coordination with HERS TAC to integrate information needs of EEM with work process and output of HERS and/or energy efficiency certification. 
Issue Paper No.: 4

Name of TAC: $\quad$ EEMs

Contact person: Walter Patterson, Texas Energy Extension Scrvice

Date of issue paper: Novemher 2, 1991

Title of issue: $\quad$ Should debt-to-income ratio adjustment in a uniform EEM be accomplished hy fixed ratio increases or scaled based on the building's energy performance?

Recommendations (if any) (include majority/minority): The uniform EEM should use both fixed ratio increases and incremental ratio adjustment. For homes that meet a nationally recognized, voluntary, consensus energy standard; debt-to-income ratios should be increased a fixed amount. Additional adjustment of the horrower credit worthiness aspect of mortgage underwriting should be accomplished by crediting additional energy savings (measured from the national standard benchmark reference) to the expense side of the debt-to-income ratios. For existing homes not requiring improvement at time of sale or refinance, energy savings (measured from the national standard benchmark reference and either positive or negative) should be credited to the expense side of the debt-to-income ratios. For existing homes being improved at the time of sale or refinance, energy savings of proposed energy improvements should be credited to the expense side of the debt-to-income ratios.

Summary of analyses already completed: The 1990 Housing Act requires HUD to establish minimum efficiency requirements for government insured lending at a level equivalent to a specified model energy code. Fannie Mae has announced increased ratio lending for new homes constructed to the same model code. These actions suggest establishment of a national benchmark from which performance based energy improvements can be measured and the resulting savings be allowed to amortize the added cost of including energy improvements in conventional and government insured mortgages. This method of borrower credit worthiness adjustment is called PITI-ES. This method fosters energy efficiency by allowing the savings of energy improvements to be directly credited in a "science" adjustment of the underwriting process.

Alternative solutions with advantages and disadvantages:

Alternative 1. PITI-ES.

Advantages:

1. Increased ratios for a prescribed level of efficiency provide a uniform benchmark from which the savings of higher efficiency levels can be measured and equitably credited to the borrower's ability to meet the added debt service of the more efficient and more expensive house.

2. Provides a mechanism to accommodate new energy savings technologies.

3. Guards against overimproving by virtue of the trade-off between added deht service vs. energy savings.

4. Removes the judgement or underwriting "art" burden on mortgage underwriters who are unfamiliar with the technology of energy efficiency.

Alternative 2. Increased ratios alone for prescribed efficiency levels.

Disadvantage: Locks in the prescribed level as an informal limit on energy efficiency. 
Alternative 3. PITI + E (principal, interest, taxes, insurance + energy costs)

Disadvantage: Administratively impossible because of the absence of a single amount that represents the nationally applicable energy operating cost adjustment to he added to hase deht-to-income ratios.

Research still needed (if any): None. 
Attachment to Issue Paper No. 4

\section{Borrower Credit Worthiness Issue of an Effective Energy Efficient Mortgage}




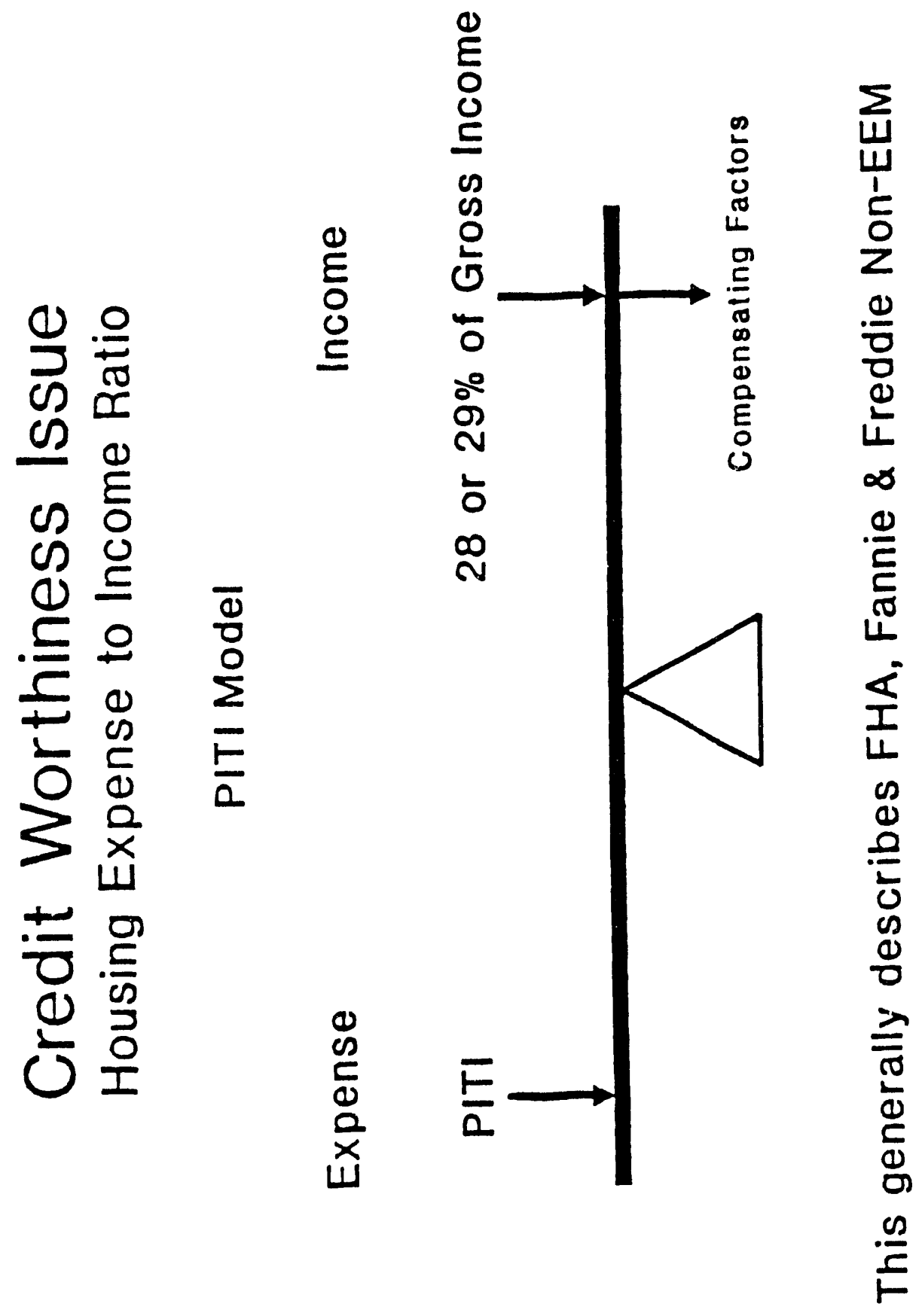




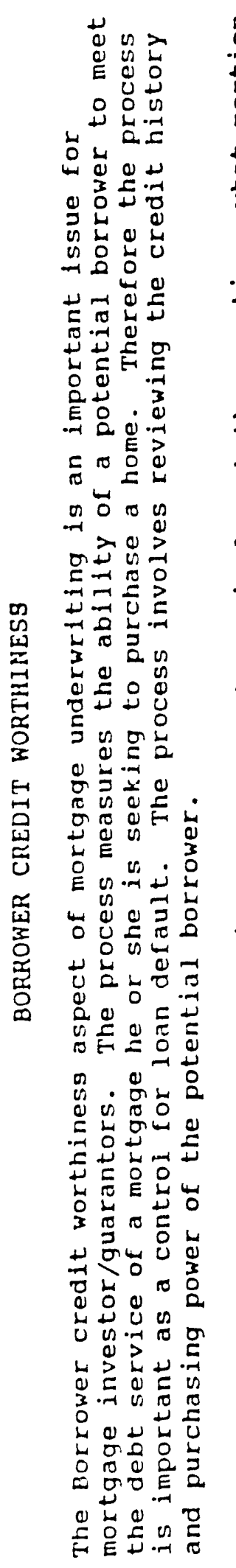

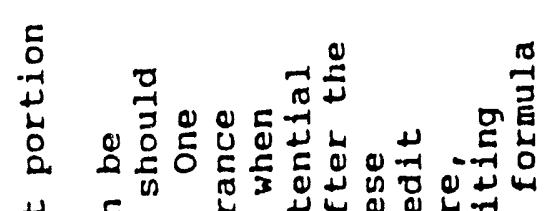

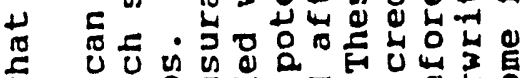

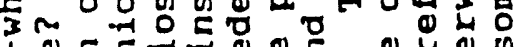

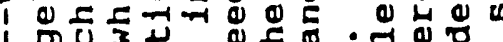

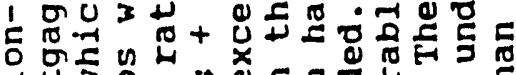

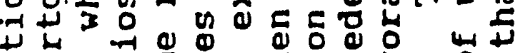

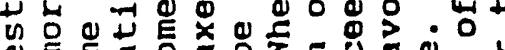

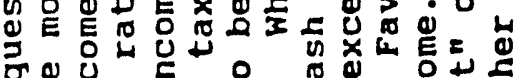

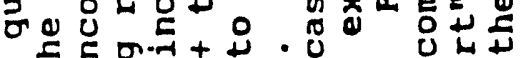

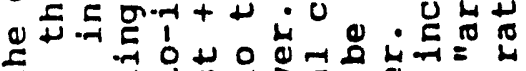

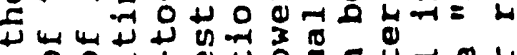

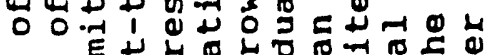
苟 \% 0 ठ

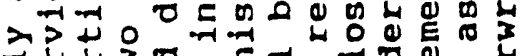

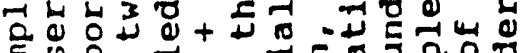

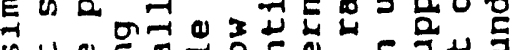
ต

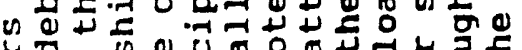

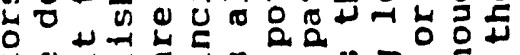

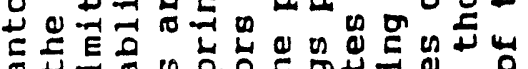

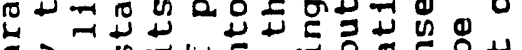

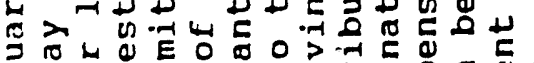
бू卩

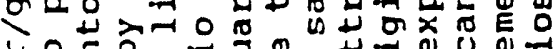

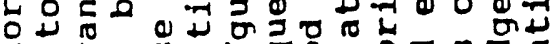

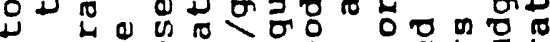

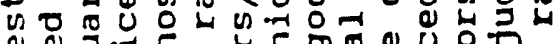

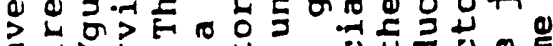

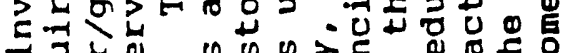

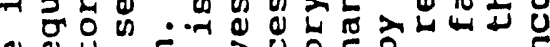

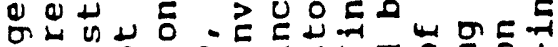
ס山

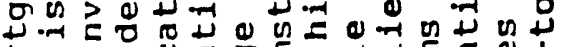
H

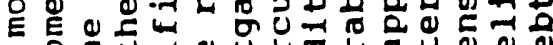

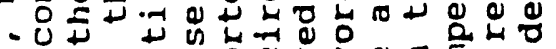

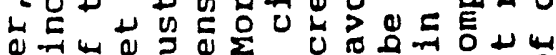

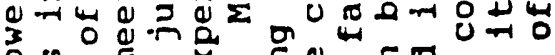

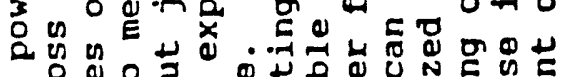

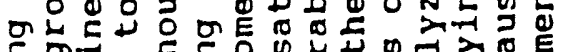

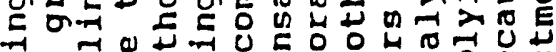

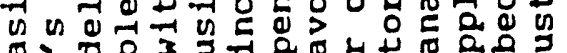

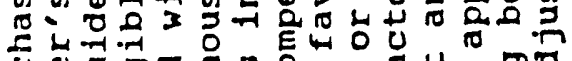
论

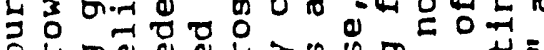

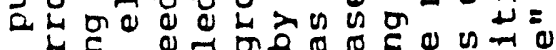

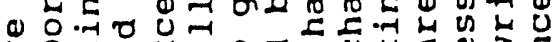

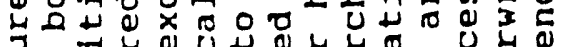
的的可

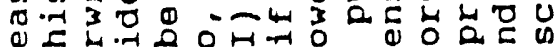

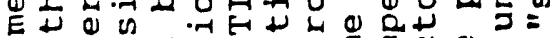

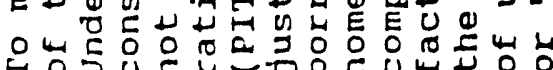

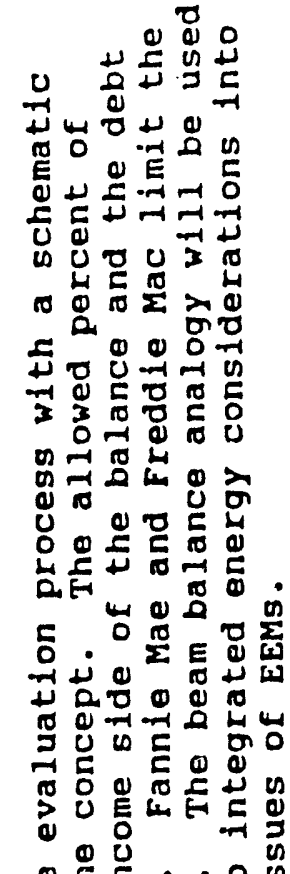

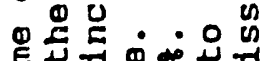

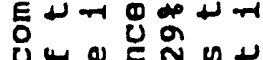

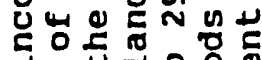

1́ ○。ำ

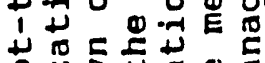

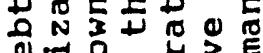
ช

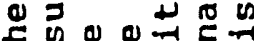

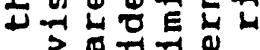

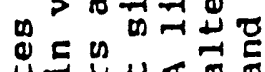

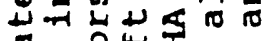

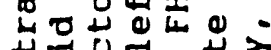
政

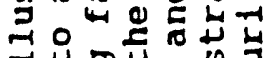
$\rightarrow$ 我 4

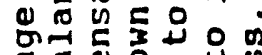
ㅁำ

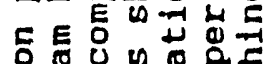

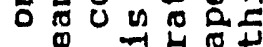

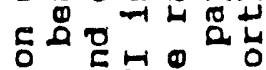

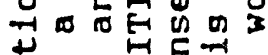
幽出造造 पि口

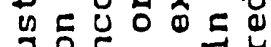
ว च牙先 $\rightarrow$.

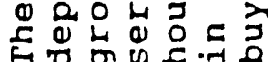

पू

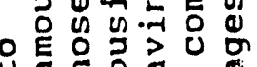
十

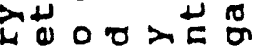

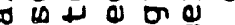
車

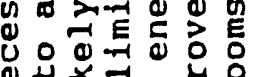

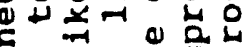
g团山出出

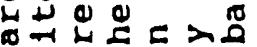

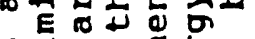

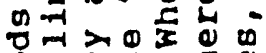

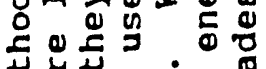

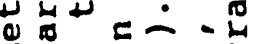
E 的正出留各 可出

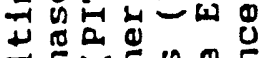

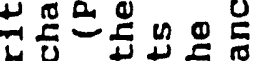
3 近团

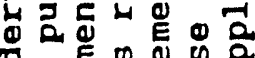
O E

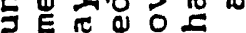

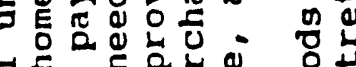

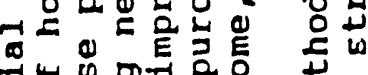
式出饮 四

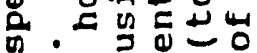

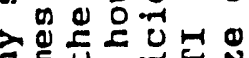
政证 足。出出而

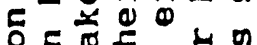

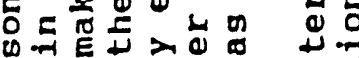

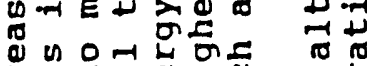
때플

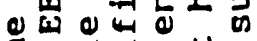

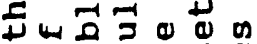

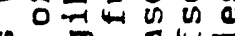
(1)

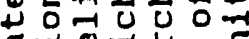

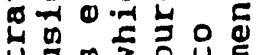

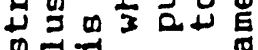

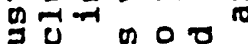
$\exists$ 둥 $\exists=$ Uै

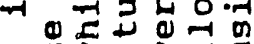
-

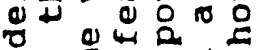
造 E

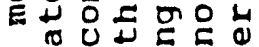

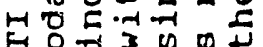

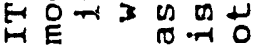

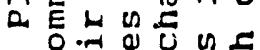

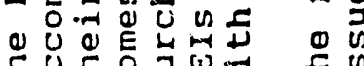

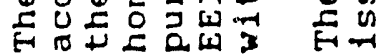

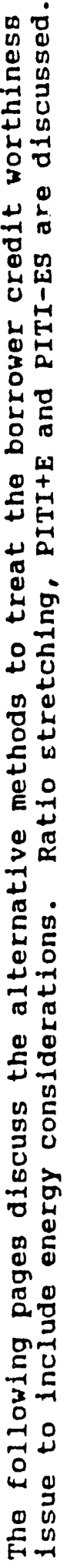




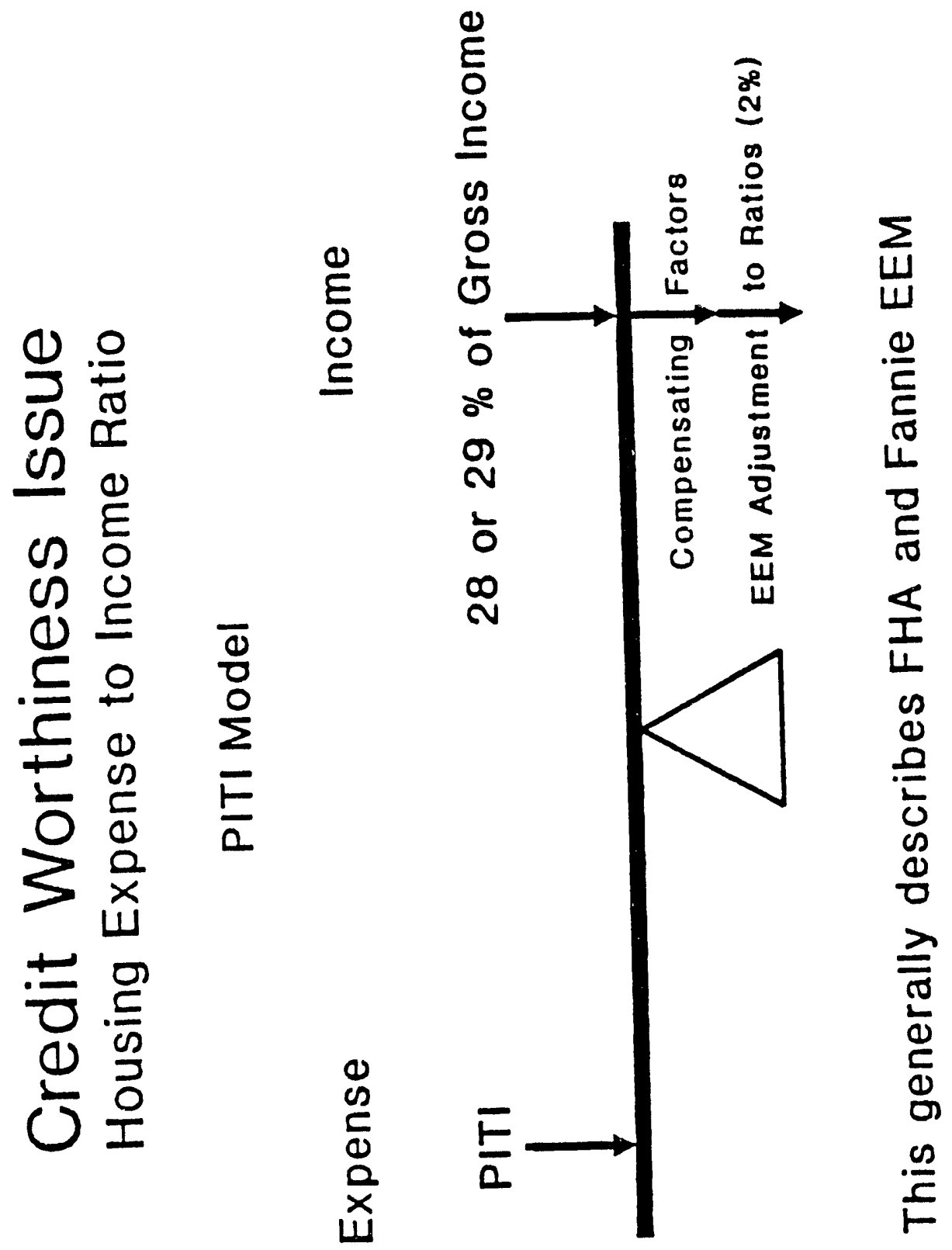




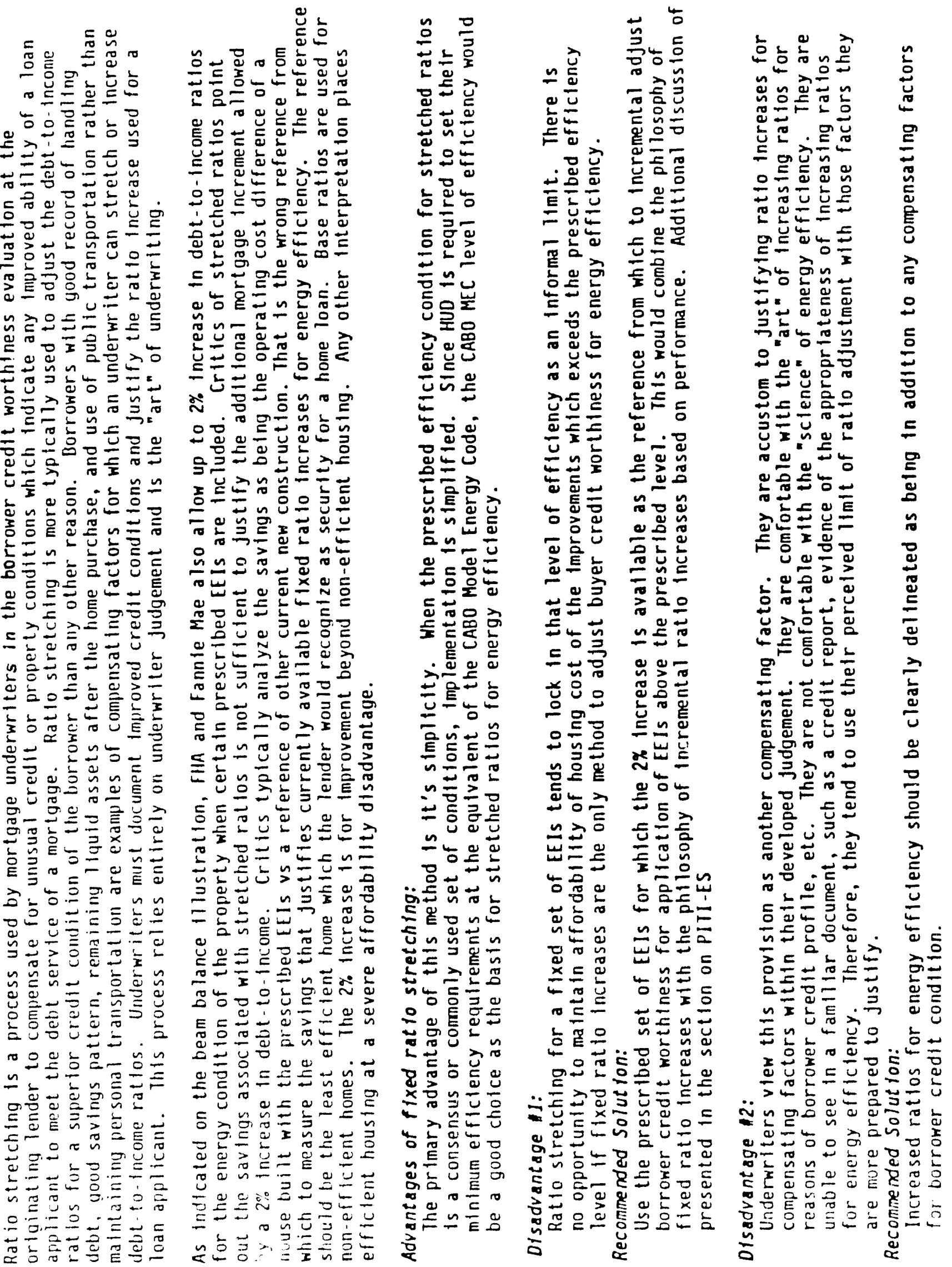



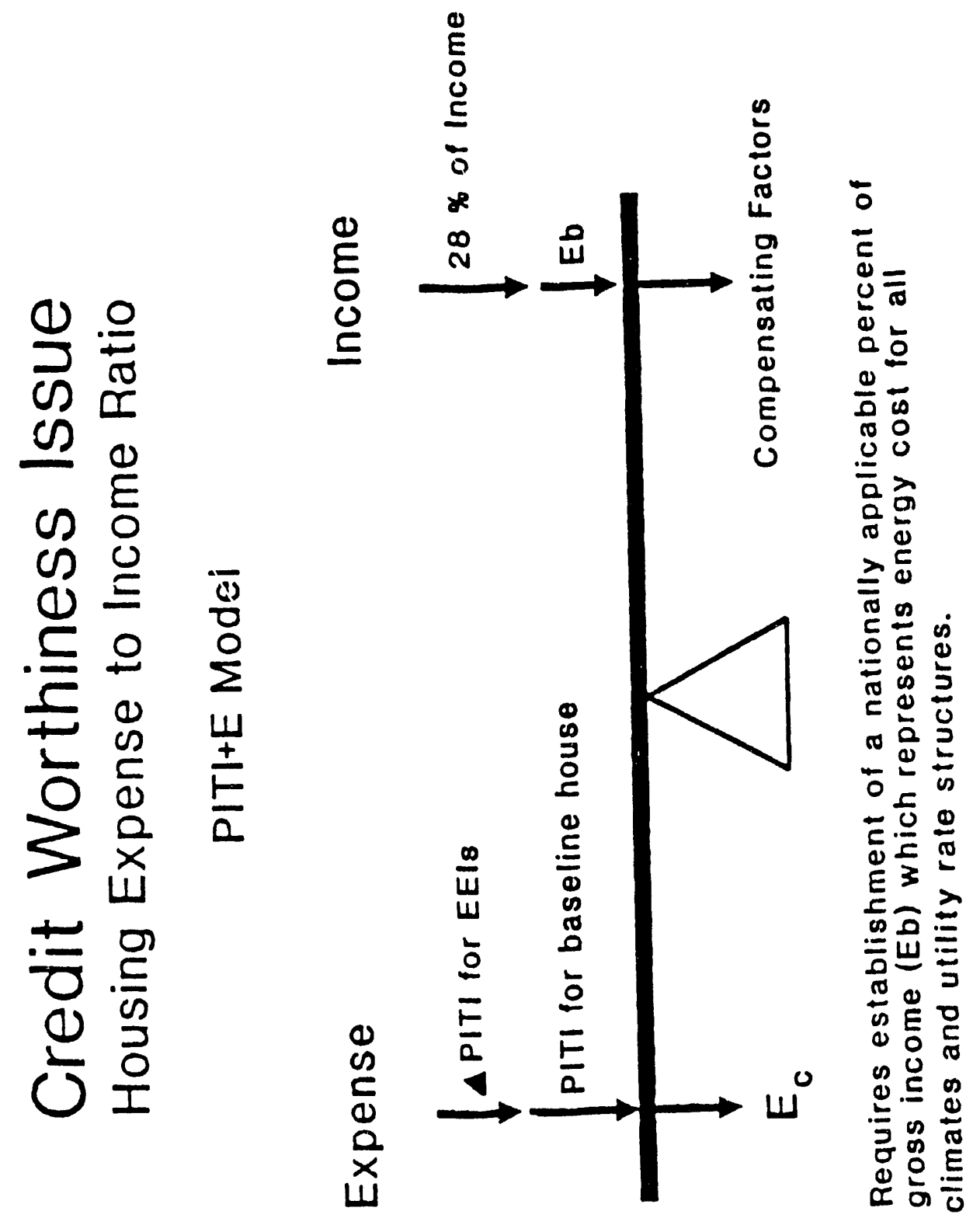


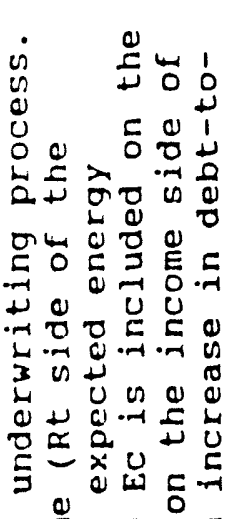

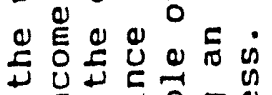

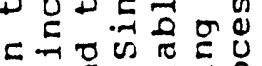

$5{ }^{-1} 0$ in

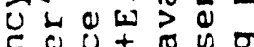

व

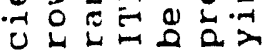

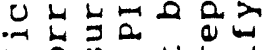

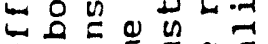

0.

के.न is 0 क

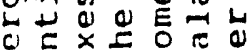

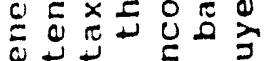

ن

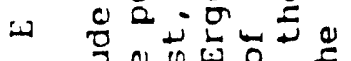

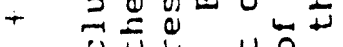

$\exists \quad . \quad 0$

- 1 ○ी

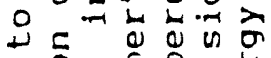

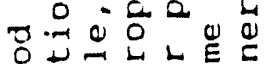

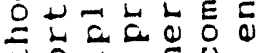

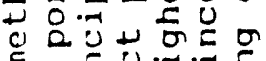

$E$ U.

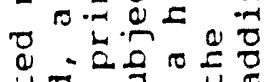

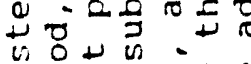

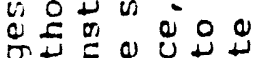

б山े

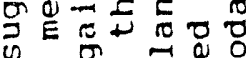

入四范范

元云几

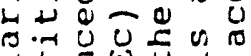

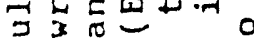

田出出

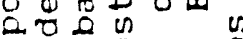

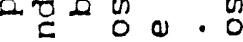

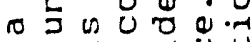

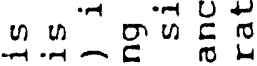

도워

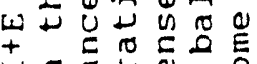

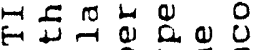

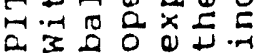

站

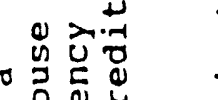

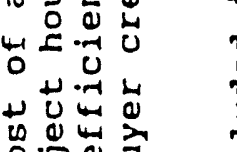

U

क人 ⿰彳

لد

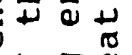

녕원

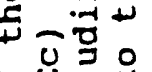

国步

-

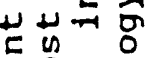

0 0

$3>20$

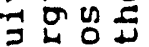

бण

वे

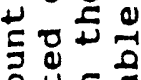

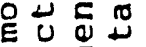

\%

ᄃ

$\approx$ व

-

की

논

o

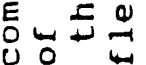

U०

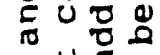

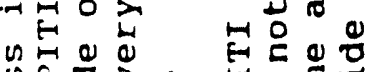

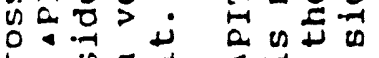

出为光

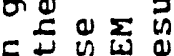

$\sum \omega$

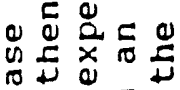

幽 0

过造些

- 0 O

०

\&

和䟚

$\because$ - o o

तन

牙

岂㟔嵒嵒

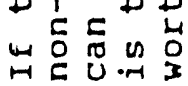

5

め岂

خे

告

点氙

$\overbrace{-1}^{\infty}$ on

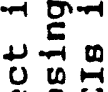

ํํㄹ

ํํㅇำ

㟧

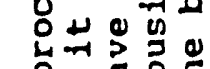

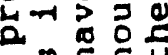

正导

0 可㞻

可 的穴

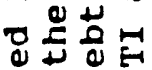

ป لَّ

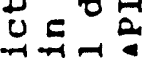

बे

농

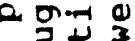

त

с

出的些

品号号

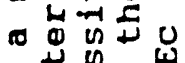

का

पू

0 o $=$ व

尖

否导至

0

3 送正

ญ品 Е

도엉

会炅包

院岂

0 - 0

ป嵒元

ᄃ

لِّ

空茴它
-1 20

H.

島足弌式

ong

氙告出

万斥它

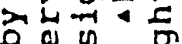

0 जू

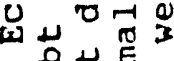

Oण =

का

ว

ชั

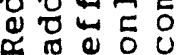

告

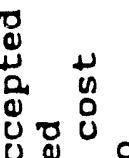

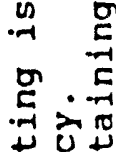

is

出

원

入告

की

of

造宫

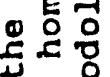

$\infty \frac{8}{5}$

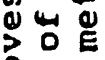

En

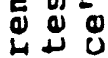

ט

w

D东

ब。

旨唡

넘 동

웡

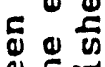

$\Phi=$

岁讪

प

당

ज包

क ल

욤

武要

T.

㟧艺

ำ

舟=

式苗

西哲骂

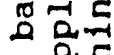

แ

a

可了

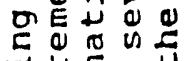

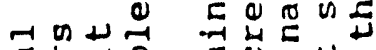

뭉

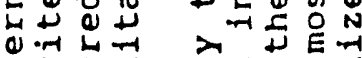

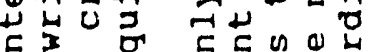

开山上

๑ ه

응 is is

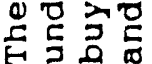

o $40 \pm 0$

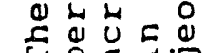

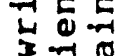

UE

嗐

总宗是

ơ

प्र

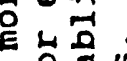

4 吾

०

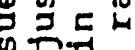

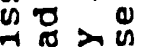

o 0 넘

四

1

计

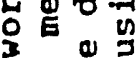

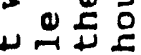

चै

ه广

บै 일

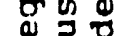

ण

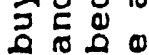

(1) o 0

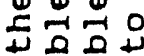

$=\vec{x}$

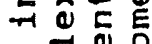

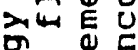

का

员员呈。

ED

象是㟔崖

ठู

ह

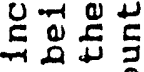

㟧趽斯

व 0

兵范

ब出

o

-1 $0 x$

点品员 

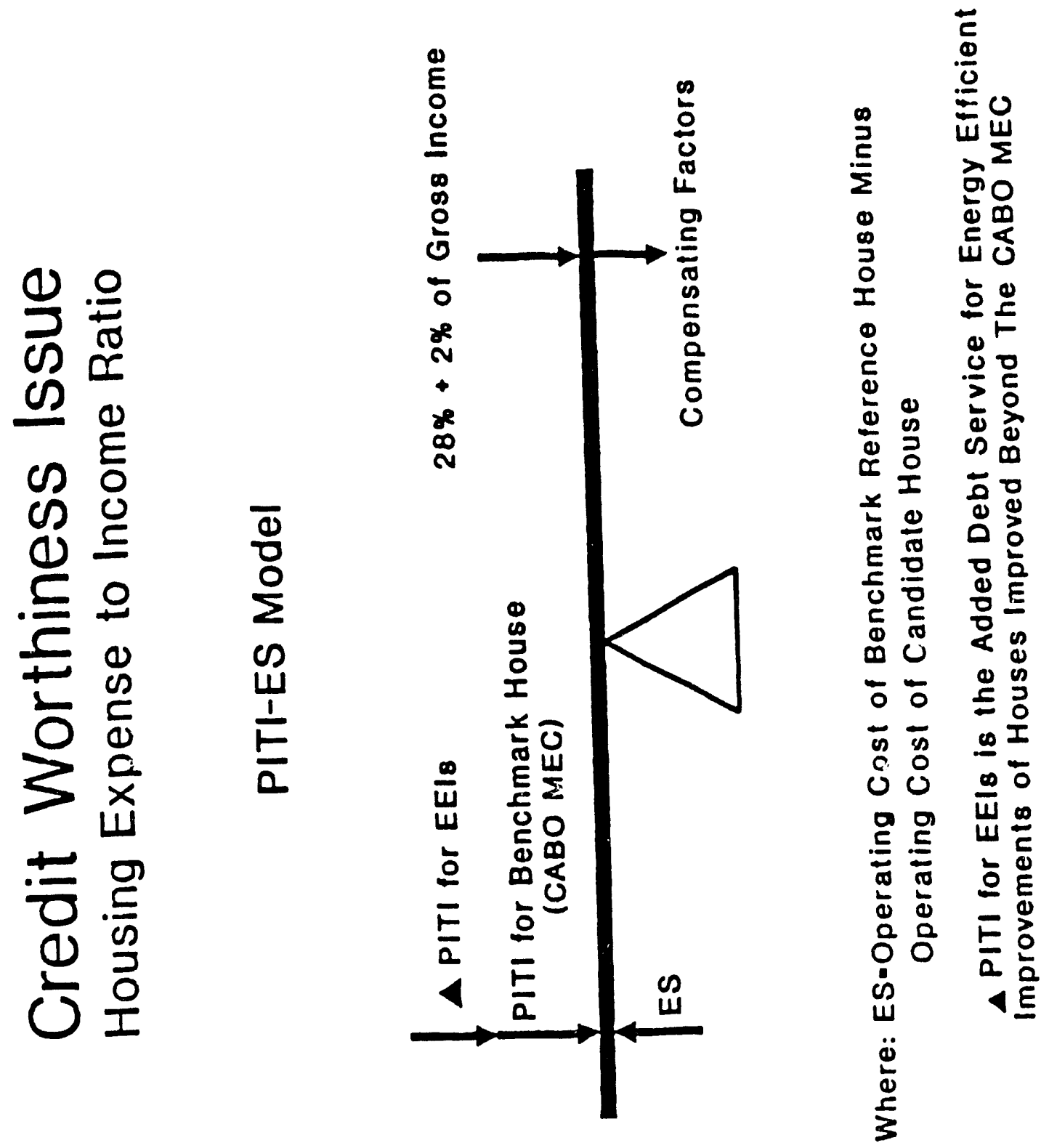


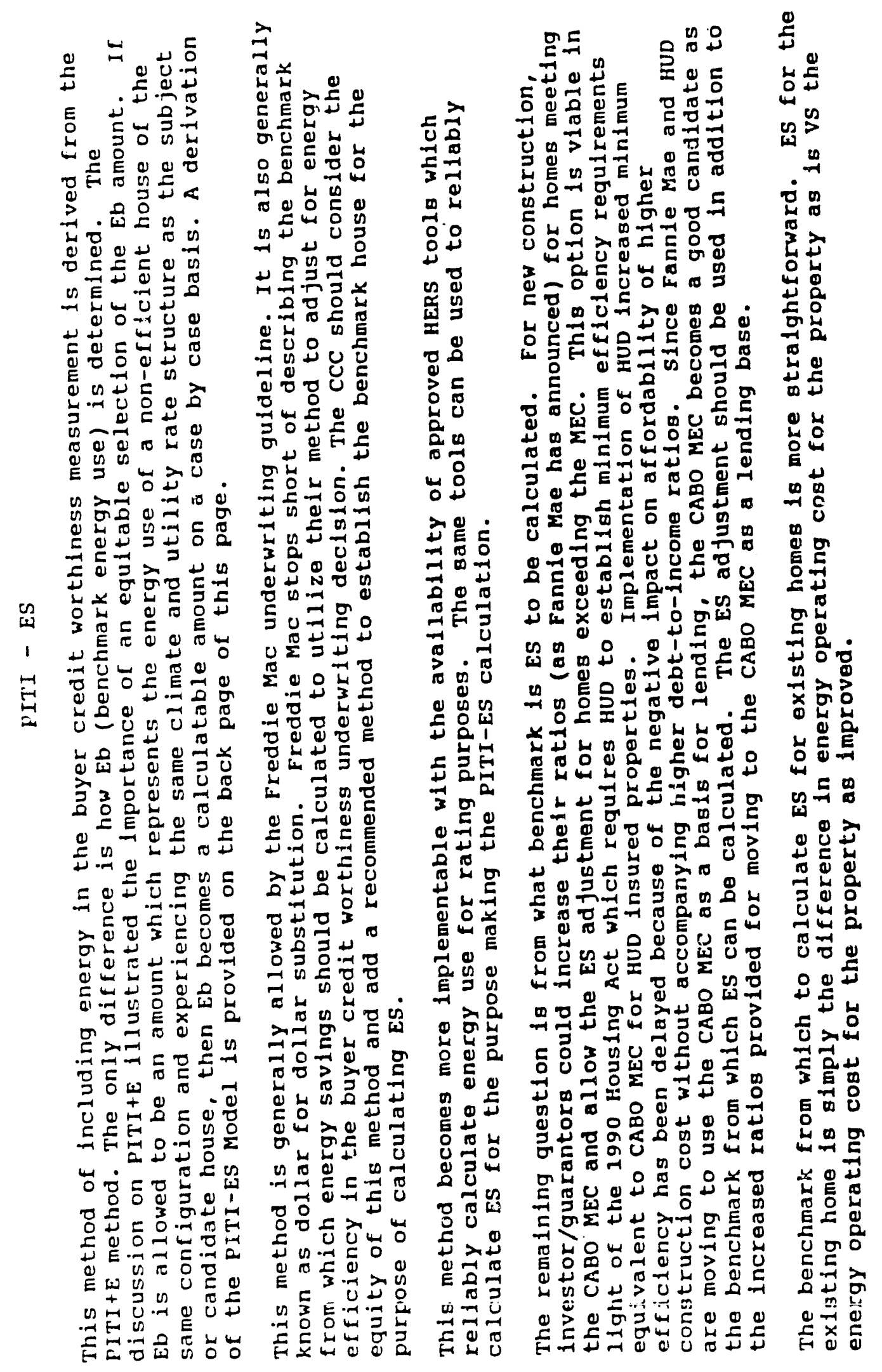



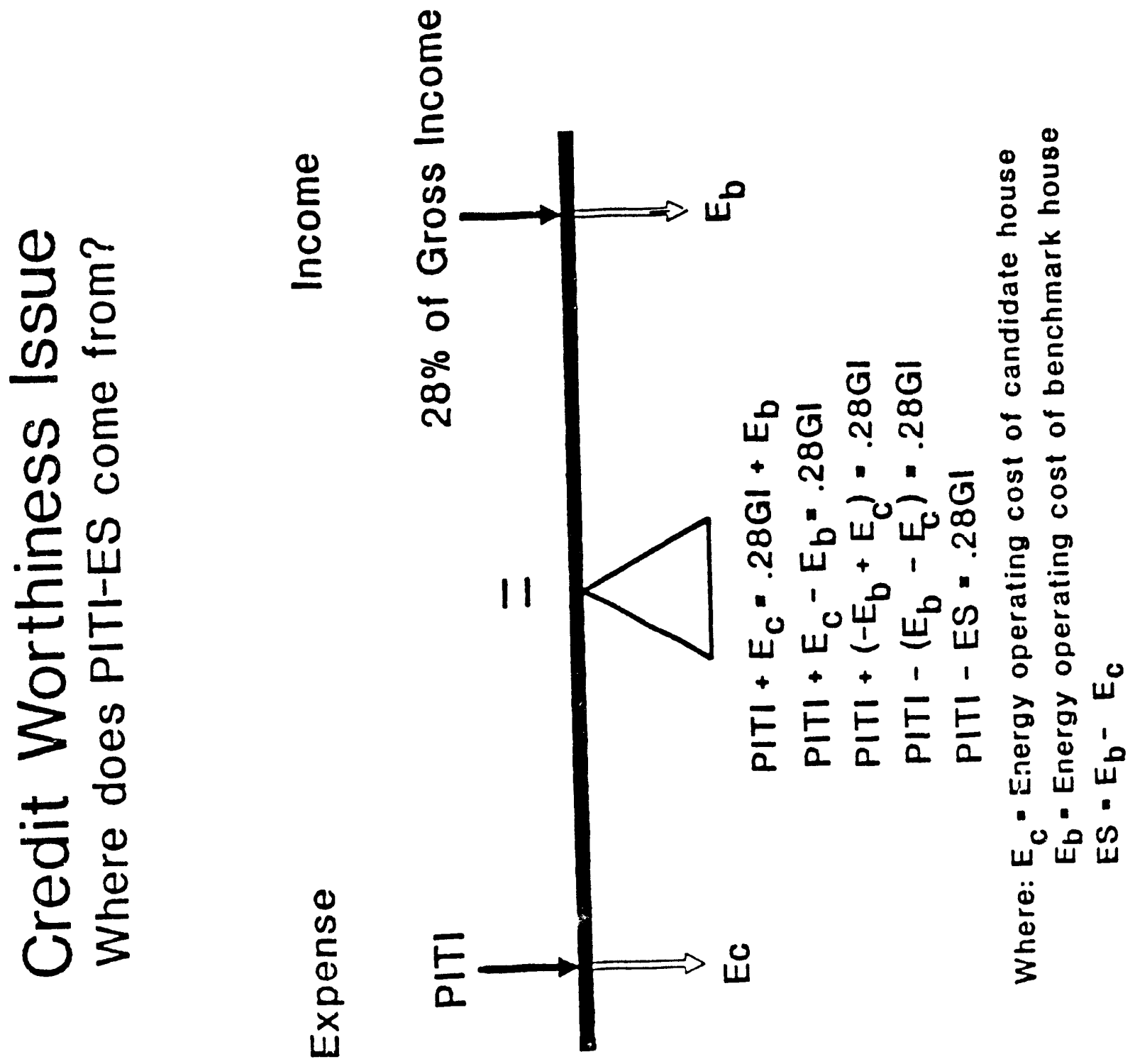


\section{Issue Paper No.: $\quad 5$}

Name of TAC: $\quad$ EEMs

Contact person: Bill Brewster, Mortgage Bankers Association

Date of issue paper: Octoher 8, 1991

Title of issue: Is it desirable to have a uniform EEM program and, if so, what are its characteristics? What underwriting rules should apply to EEMs? What should the qualifying ratios be? Should a ratio stretch or a PITI + E approach he used? If the debt-to-income ratios are increased for mortgages having energy-efficient housing, what should the ratios be? One specific amount or scaled to reflect the building's energy performance? or income level? What should the ratios be for given household incomes and/or energy performance levels?

Recommendations (if any) (include majority/minority): The agencies are required under Section 946 of PL 101-625 to work toward "a uniform plan to make housing more affordable through energy efficiency." The TAC recommends that the agencies utilize the specific amount of energy cost savings when incorporating energy into the underwriting process.

Summary of analyses already completed: Currently, each agency has a different approach to EEMs just as each has a different approach to underwriting all mortgages. It is not practical for a uniform EEM to dictate specific qualifying ratios, approaches, etc. To the extent that each agency is open to adopting additional and more flexible approaches to EEMs, consumers and real estate practitioners will find EEMs more attractive.

Energy Efficient Mortgages: Proposal for a Uniform National Program, The Alliance to Save Energy, August 1989.

Guidelines for a Uniform Energy Efficient Mortgage Program, prepared for the U.S. Department of Energy by the Uniform Energy-Efficient Mortgage Steering Committee, Palo Alto, California, March 1, 1989.

Current credit guidelines of Fannic Mae, Freddie Mac, VA, FHA, Farmer Home Administration, and the Vermont Housing Finance Agency. 
Issue Paper No.: 6

Name of TAC: $\quad$ EEMs

Contact person: Bill Brewster, Mortgage Bankers Association

Date of issue paper: October 8, 1991

Title of issue: $\quad$ What data or analyses have been done (or are needed) on the impact of energy improvements on resale value of a house? What would a uniform appraisal/valuation method be? How does one measure the value of energy efficiency improvements? How should they be accounted for in the appraisal process?

Recommendations (if any) (include majority/minority): The TAC recommends that information on the energy efficiency of a home be made as widely available to appraisers as possible and that they be required to consider its impact on value.

Summary of analyses already completed: The actual resale value of energy improvement, depends on local consumer demand. National surveys indicate consumers are willing to pay more for energy-efficient housing. Historical sales data verifying this demand are not currently available in most markets. The sales comparison approach to value is preferred by the federal housing and banking agencies. It is also preferred by appraisers, lenders, and mortgage insurers.

The Energy Efficient Mortgage: A Lender-Appraiser Manual, The Idaho Energy-Efficient Mortgage Program, March 1990.

"Incorporating Energy Efficiency into Residential Appraisals," Paul L. Hendrickson, The Real Estate Appraiser and Analyst, Summer 1989.

"Measuring Home Energy Efficiency," Lantz L. Rakow, The Real Estate Appraiser and Analyst, Summer 1989.

"Adjusting for Energy Efficiency in Residential Appraisal," David E. Levy, The Appraisal Jourmal, July 1987.

"Residential Appraisal: A Behavioral Approach to Energy Efficiency," James R. DeLisle, The Appraisal Journal, July 1984.

"The Energy Mortgage Value Method," Steven J. Foute, The Real Estate Appraiser and Analyst, Spring 1982.

"Measuring Energy Efficiency for Selection and Adjustment of Comparable Sales," John B. Corgel et al., The Appraisal Journal, January 1982.

"The Impact of Energy on Value," Gordon T. Brown, The Real Estate Appraiser and Analyst, NovemherDecember 1980.

"The Influence of Solar Energy Systems on the Value of Dwellings: Theory and Practice," James R. Webb, The Real Estate Appraiser and Analyst, January-February 1980. 
"Residential Energy Capitalization Technique and Single-Family Home Underwriting," Wade R. Ragas and Jack M. Wyatt, II, The Real Estate Appraiser, January-February 1978. 
Issue Paper No.: 7

Name of TAC: $\quad$ EEMs

Contact person: Jane Stockinger, Federal Home Loan Mortgage Corporation (Freddic Mac)

Date of issue paper: August 15, 1991

Title of issue: What should the total amount of energy improvements he? (This issue paper addresses hoth new and existing homes.)

Recommendations (if any) (include majority/minority): All agencies agree that there must he some type of limit on the total cost of energy-efficient improvements that will he considered under an EEM program.

Summary of analyses already completed: When securing a loan with residential real estate, the underwriting decision as to whether the subject property is adequate security for the loan, and whether any consideration should he given for certain improvements, must he based on the market value and the marketahility of the property.

Proper loan risk evaluation requires that any credit to be given for improvements must be reflected in the appraised value. No improvement adds $100 \%$ of the cost to the market value, and some improvements may overimprove the property so that the improvement actually adds little or no value to the property.

Therefore, to receive credit for energy-efficient improvements in the loan amount, the value of such improvements must he reflected in the appraised value, and the improvements should not overimprove the property.

\section{Alternative solutions with advantages and disadvantages:}

$\underline{\text { Snecific dollar limit }}$

Advantages-easily understood and no calculation required.

Disadvantages-the size of the home may affect the cost of improvements, and purchasers/owners of larger homes may be penalized. There are different levels of energy-efficient improvements, i.e., minimum to bring a home to a high energy-efficient rating, or more extensive improvements such as solar heating/cooling systems.

Sten dollar limit (i.e., lower dollar limit for minimum improvements, higher dollar limit for more extensive improvements)

Advantages-lakes into account the disadvantages of the specific dollar limit.

Disadvantages-unless a clear explanation of what the standards would he for the steps, EEM participants will have uncertainty when applying the limits.

\section{Percentage of loan amount}

Advantages-casy to understand and calculate.

Disadvantages - the loan amount is not directly relevant to whether a home is or is not energy efficient. Borrowers who make higher downpayments and who are considered lower risks, would have less money availahle for energy-efficient improvements than the higher loan-to-value ratio, riskier horrowers. 
Research still needed (if any): To arrive at a rational, quantitative limit, we need dita on the average costs of installing the various levels of energy-efficient improvements, and the datta must lake into account the size of the home and the geographic arad. If the step dollar limit is preferred, then data will be needed to determine what type of energy-efticieni iniprovements are needed for each level. 
Issue Paper No.: 8

Name of TAC: $\quad$ EEMs

Contact person: Jane Stockinger, Federal Home Loan Mortgage Corporation (Freddic Mac)

Date of issue paper: August 15, 1991

Title of issue: What should the total loan-to-value ratio of EEMs be?

Recommendations (if any) (include majority/minority): All agencies agree that energy-efficient mortgages must and should be treated under standard loan-to-value (LTV) ratios and no special consideration in the form of higher LTVs can or should he given.

Summary of analyses already completed: For VA, there is no LTV calculation since qualified veterans may obtain $100 \%$ financing. The areas of the cost of the improvements and their effect on market value is the issue.

For FHA, maximum LTV ratios for loan programs are determined hy statute.

For Fannic Mae and Freddie Mac, the maximum LTV ratio for any loan purchased is $95 \%$. This ratio is regulated by the agencies' charters, which were established by Congress.

For conventional loan programs, the agencies may reduce the LTV for riskier products or borrower characteristics, and under no circumstances may the LTV for any loan exceed $95 \%$.

Additionally, LTV ratios are used extensively in the secondary and primary mortgage markets to establish pricing, loss reserves, and a myriad of other figures. Separate loan-to-value ratios for EEMs, even if they were allowed by agency charters, would wreck havoc in the industry if the program generated high loan volume.

Therefore, specific underwriting and appraisal guidelines for EEM programs is the only option available.

Alternative solutions with advantages and disadvantages: None.

Research still needed (if any): None. 
Issue Paper No.: $\quad 9$

Name of TAC: $\quad$ EEMs

Contact person: Jim Curtis, Association of Energy Efficient Mortgage Service Companies

Date of issue paper: August 15, 1991

Title of issue: $\quad$ For existing huildings, how should (or how can) energy improvements be financed? As home improvement loans? Or in the first mortgage? If the latter, how does the current owner who wants to make improvements finance them?

For existing homes (i.e., buildings) there are several loan types and programs available to homeowners and home buyers who wish to finance the cost of needed energy improvements. The most common loan types are:

1. Conventional Home Improvement Loans: These loans are for home improvement work such as remodeling, additions, and/or energy improvements. Generally, they are for less than $\$ 10,000$, with repayment periods of less than 10 years. They are normally unsecured. Under the current tax laws the interest on a conventional home improvement loan is not tax deductible.

2. Home Equity Loans: These loans let you (as a homeowner) use the equity in your home as collateral and thus have a somewhat lower interest rate than an unsecured conventional home improvement loan. In recent years, home equity loans have largely replaced home improvement loans. Under the current tax laws, interest on a home equity loan is tax deductible provided the loan is for no more than the original purchase price of the home and the documented value of the home improvements.

3. FHA Title 1 Loans: These loans are available to all homeowners in the United States. They are for major rehabilitation work. The maximum interest rate (currently $14.00 \%$ ) is set hy the federal government (i.e., HUD). Title 1 loans offer longer repayment periods than conventional home improvement loans-up to 15 years-with loan amounts up to $\$ 20,000$. You can combine energy improvements with rehabilitation work on a Title 1 loan. If and when you are interested in this type of loan, you must specifically ask to apply for it.

4. VA Home Improvement Loans: Armed Forces Veterans who are homeowners-who live in the home-and have a good credit rating are eligible for this VA home improvement loan. Repayment of the loan is insured by the U.S. government. The maximum loan amount is currently $\$ 25,0(0)$, with terms up to 30 years. The current interest rate is $12.5 \%$. But very few lenders or hanks offer these loans. Veterans should contact their local VA office for the names of local lenders and local rates.

5. Additions to the First Mortgage (The Energy-Efficient Mortgage (EEM) Concept): When you are buying a home (or as a homeowner when you are refinancing your existing mortgage) you have an excellent opportunity to finance needed energy improvements by adding the cost of the improvements onto the new first mortgage. The added amount will normally be a small fraction of the total mortgage and will normally immediately pay for itself through reduced energy bills. Adding the cost of energy improvements to a first mortgage allows you to spread out the payments over 30 years, while you benefit from lower energy hills right away.

Note: The immediate and long term "cash flow" of savings versus loan payments should he a major consideration when a home buyer or homeowner is deciding how to acquire (i.e., pay for or linance) needed energy improvements. 
Public Programs (Subsidized Loans): Low interest loans are often available through state and local governments and utility companies. To find out ahout current programs, call your city government or utility company.

Low Income Assistance: Free weatherization is available to houscholds that meet income guidelines. Check your town or cily government or local community action agency about the Weatherization Assistance Program in your area. 
Attachment to Issue Paper No. 9

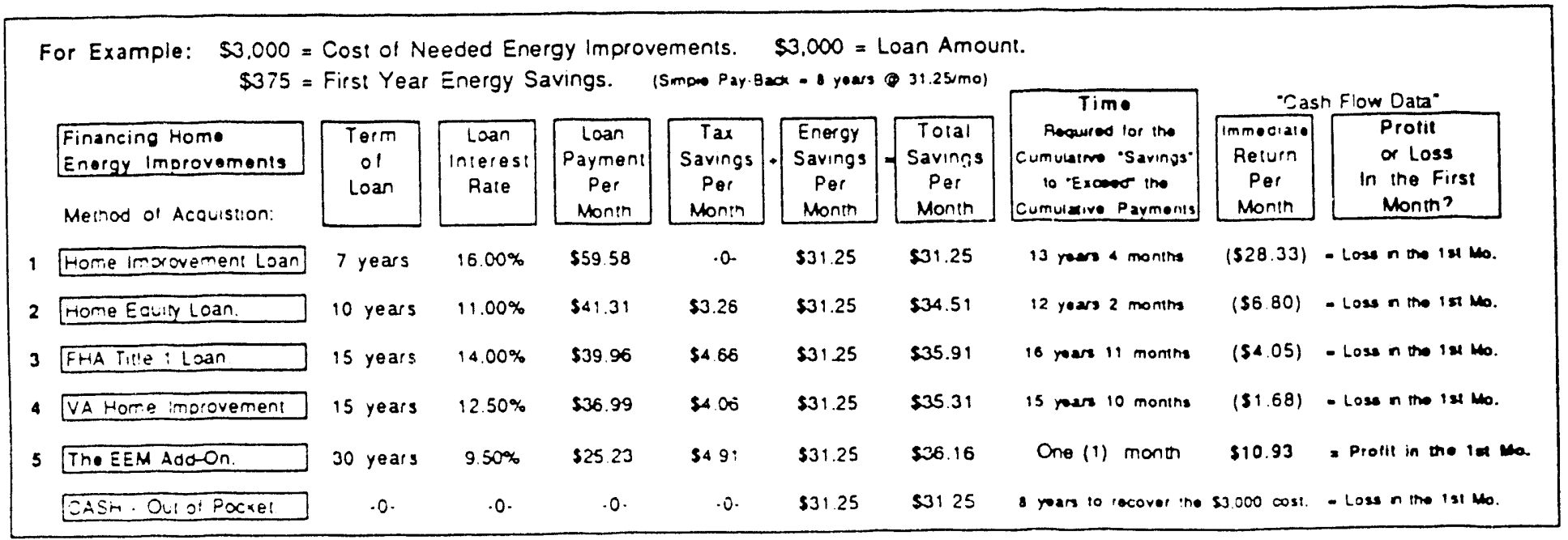


Issue Paper No:: 10

Name of TAC: $\quad$ EEMs

Contact person: $\quad$ Richard Faesy, Energy Rated Homes of Vermont

Date of issue paper: Octoher 3, 1991

Title of issue: $\quad$ Avoidance of delay in loan approval process

Recommendations (if any) (include majority/minority): Timeliness of HERS information is of the utmost importance in order to avoid any delay when utilizing an EEM. Therefore, as early as possible in the home sale process, preferably at or hefore the listing of a property, provide the opportunity to obtain:

- an energy rating with energy costs and savings

- a list of recommended energy improvement measures (if any), and

- the contractor-installed cost of these improvements.

Summary of analyses already completed:

1. Guidelines for a Uniform Energy Efficient Mortgage Program, March 1, 1989, Prepared for the U.S. D.O.E. under Contract 021376-A-K1.

2. Collaborauve Consensus Committee of Energy Efficient Mortgages and Home Energy Rating Systems, EEMs Technical Advisory Committee, Potential Issues for Discussion, White Paper by the Alliance to Save Energy. 
Issue Paper No.: 11

Name of TAC: $\quad$ EEMs

Contact person: Tom Farkas, Edison Electric Institute

Date of issue paper: Oc:oher 7, 1991

Title of issue: What kind of information is needed by lenders to trigger an energy-efficient mortgage? What hasic quantities do the mortgage lenders need from the HERS?

Recommendations (if any) (include majority/minority): Mortgage lenders need a rating model that is hased on nationally recognized consensus standards and that will, in a uniform and consistent way, directly measure the energy cost savings of the candidate house ${ }^{5}$ as compared to the same house at its standard reference level; and if necessary, measure the energy cost savings of the candidate house compared to the same house with energy efficiency improvements added.

Summary of analyses already completed: To obtain the energy cost savings, the following is done. The candidate house's energy use ${ }^{6}$, in Btus per year, is calculated using recognized consensus energy calculation tools. Then the energy use of the same house at the standard reference level is calculated using the same method. The energy cost of the candidate house as it exists and at its standard reference condition are obtained by multiplying the respective energy uses by the local utility rate. The energy cost savings, if any, will be the difference between the two. If the candidate house as it is compared to itself at standard conditions creates neutral or positive energy cost savings, then the buyer of the candidate house would be eligible for an energy-efficient mortgage. If there is no net positive energy cost savings hetween the candidate and its reference, then the house is considered as not energy efficient. Then the rater of the house should identify a package of cost-effective energy improvements that could be included in the mortgage, thereby creating an energy-efficient mortgage that provides for energy efficiency improvements to be made. To determine if the improvements are worthwhile and cost-effective, the energy cost savings due to the improvements would be calculated in the same manner as shown above (comparing the house as it exists to the house as it would be after energy-efficient improvements are completed) and used in the mortgage evaluation.

Alternative solutions with advantages and disadvantages: Advantages-Based on nationally recognized consensus standards; directly measured, consistent, accurate, and uniform: fuel neutral; keeps energy use and cost at comparable level with all other housing components and their costs at the location that the mortgage is heing applied (cost of improvements and energy cost savings will accrue at the house); provides only meaningful information of comparison; eliminates arbitrary indirect approaches; does not artificially depress energy efficiency potential nor preclude incorporation of advanced technology benefits; and allows for a reporting option to characterize the energy efficiency of that house in easy to understand nondimension (percentage) terms, e.g., the ratio of candidate and reference house energy use and/or cost.

Disadvantages-(0-1(0) Scale) Arhitrarily defines zero efficiency and zero energy use house; arbitrarily compresses efficiency po:ential; hased on marketing approach of fuel efficiency not nationally recognized

${ }^{5}$ Candidate House means the house to he purchased with the mortgage.

${ }^{6}$ Energy use means the energy that is used at a huilding or facility and measured in terms of energy delivered to the huilding facility. (As defined in Executive Order 12759) of April 17, 1991. See FR Vol. 56, No. 76, April 19, 1991.) 
consensus standards appropriate for the standard reference house and what level of energy cost savings are appropriate for an energy-efficient mortgage. 
Issue Paper No.: 12

Name of TAC: $\quad$ EEMs

Contact person: $\quad$ Chip Coffay, Fannie Mae

Date of issue paper: August 15, 1991

Title of issue: What kind of information is needed by lenders to trigger an energy-efficient mortgage? What basic quantities do the mortgage lenders need from the HERS?

\section{Recommendations (if any) (include majority/minority):}

- Need Department of Energy (DOE) to provide the following information:

- establish and maintain a state by state list of acceptable/eligible energy efficiency improvements that can be included in an energy-efficient mortgage;

- establish and maintain an annual, state by state, average cost of energy per square foot of gross living area.

- Need the HERS to provide the following information:

- a rating of the overall energy efficiency of an existing house;

- a method of applying the HERS rating to the average cost of energy per square foot of gross living area to result in an estimated dollar cost of energy expense for a house (on a monthly basis);

- a projection of the impact on the existing energy efficiency rating of a house for each of the eligible improvements on the DOE list for that state.

- Need the borrower to choose which of the eligible energy-efficient improvements are to be included in the EEM.

- Need the underwriter or HERS administer to calculate the following information:

- for retrofits: compare the estimated energy costs of the house prior to improvements with the estimated energy costs after improvements, resulting in a net cost savings per month;

- for new construction: taking the average cost of energy per square foot of gross living area for that state, estimate what the expecied cost of energy would be for a house equal to the size of the proposed construction; compare that figure with the estimated cost of energy derived from the HERS rating of the proposed construction to get a net cost savings per month.

\section{Alternative solutions with advantages and disadvantages:}

Continue to use pass/fail rating system tied to an increase in the qualifying ratios.

Advantage(s): easy to administer and apply.

Disadvantage(s):

- does not distinguish hetween degrees of́ ciniciency

- pays no attention to actual energy savings

- impat of ratio increase is tied to income level rather than level of energy efficiency.

Research still needed (if any): Fuel neutral or fuel discriminating. 
Issue Paper No.: 13

Name of TAC: $\quad$ EEMs

Contact person: Jim Curtis, Association of Energy Efficient Mortgage Service Companies

Date of issue paper: August 15, 1991

Title of issue: What alternatives to escrow arrangements can be considered? And/or: Would a trustee system for fees, escrow accounts, and savings guarantees be beneficial?

escrow $n$. a contract, money etc., deposited with a third person, by whom it is to be delivered to the grantee on the fulfillment of some condition. (The Random House Dictionary 1980, by Random Heuse Inc.)

Within the existing guidelines of HUD-FHA, the DVA, Fannie Mae and Freddie Mac, Provision Number 1 of the Energy-Efficient Mortgage (EEM) Concept allows all (or a portion) of the cost of needed energy improvements to be added to a home buyer's or homeowner's first mortgage at the time of purchase or refinance.

Within the EEM procedures that have evolved to date, the funds for the energy improvements are held by either (1) the lender, (2) the title company, or (3) the escrow company.

The contractor or contractors installing the improvements are paid after the installation of the improvements has been completed.

In California, this procedural aspect of the EEM concept has not been a problem.

Proper instructions to the lender's loan file, designating exactly how much is to be held, who is to be paid, when they are to be paid, and including an agreement, signed by the contractor and the loan applicant, that they will hold the lender harmless and will "Arbitrate" any and all buyer/contractor disputes, have overcume the hassles or problems that nonparticipants have suggested might occur.

With the proper instructions to the lender's loan file-this is the one aspect of the EEM concept that works. Don't fix it.

Lenders in California who were interviewed regarding this issue are vehemently opposed to an alternative that a third party such as a utility company, local government agency, or nonprofit organization could or should be designated to administer (or escrow) the energy improvement funds.

These same lenders are hopeful that if the EEM concept is to be popularized then a HUD-FHA, DVA, Fannie Mae and Freddie Mac Uniform EEM program and procedure he implemented in the very near future.

For if an effort is to he made to make loan applicants and realtors aware of Provision Number 1 of the existing EEM type programs of HUD-FHA, the DVA, Fannie Mae, and Freddie Mac hefore a uniform EEM program and procedure is available, such an effort could, hecause of the many differences within the existing EEM type programs, complicate and add confusion to the existing loan processing procedures of lenders everywhere. And, 
Without Uniformity, the result could he a reduction in the long term utilization of the EEM concept.

The EEM Battle Cry from California is: Uniformity First-when Awareness! 
Issue Paper No.: 14

Name of TAC: $\quad$ EEMs

Contact person: $\quad$ Ken Crandall, Federal Housing Administration

Date of issue paper: August 15, 1991

Title of issue: $\quad$ How might inducements be treated within an EEM process?

Recommendations (if any) (include majority/minority):

1. Builders, remodelers, home purchasers, state and local energy agencies, energy suppliers, and others should be encouraged to allow third party financial assistance payments for downpayments and/or settlement costs to purchasers of new and existing homes that are eligible for an EEM.

2. State and local governments, nonprofit agencies, and others should be encouraged to provide grants, low interest loans and/or soft seconds for financial assistance payments on downpayments and/or settlement costs to purchasers of new and existing homes that are eligible for an EEM.

3. Federal and state agencies should allow tax credits to assist homeowners/purchasers with the cost of making energy improvements on their home.

4. HUD should allow a $5 \%$ increase in the maximum mortgage amount dollar limitation in 24 CFR 203.18 , for new and existing properties, if such an increase is necessary to account for the increased cost of the residence due to the installation of energy related improvements.

5. HUD should change its regulations, under Section 203(k), Rehabilitation Mortgage Insurance, to allow the cost of energy improvements on an existing home as a second mortgage with insurance of advances as the work is complete, with a term of 30 years (on amounts exceeding $\$ 17,500$ ).

Summary of analyses already completed: HUD agreement with Bonneville Power Authority and the Northwest Power Planning Council allows up to $\$ 1,500$ financial assistance payments for newly constructed homes. Builders of new homes can help utility companies conserve energy by providing an energy-efficient home. In turn, the utility company can provide financial incentive payments to builders and/or their home buyers. Since the money is saved by not building new facilities, the utility company can pass along the savings to the home buyer in the form of assistance with the downpayment and/or settlement costs. This would have the affect of allowing more hoine buyers to purchase new homes, thereby, helping the housing industry.

Federal and state tax credits for energy improvements would be modeled after programs established hetween 1970) and 1980. HUD currently allows a 20\% increase in the dollar limitation for solar energy systems in 24 CFR 203.18a. HUD also allows 203(k) second mortgages; however, the work must be complete prior to HUD insuring the loan. 
Issue Paper No.:

Name of 'TAC:

Contact person:

Date of issue paper:

Title of issue:
1

Implementation

Donald Buchan, Energy Efficient Builders Association

November 12, 1991

Structure of potential energy-rated mortgage (ERM) program

Recommendations (if any) (include majority/minority): The accompanying chart illustrates a potential national infrastructure for an energy-rated mortgage program. The intent of the proposed structure is to allow the maximum flexibility within state, regional, or local programs, while maintaining national standards associated with the quality assurance and information gathering aspects of the program.

The overseeing Energy-Rated Mortgage (ERM) Board would have a makeup similar to that of the current Collaborative Consensus Committee (CCC)-essentially having a broad industry base. This overall board would meet infrequently, while a smaller executive committee would meet regularly to discuss program policy.

Advisory groups, similar to the current technical advisory committees to the $\mathrm{CCC}$, would he available on a "standing" basis or on an "as-required" basis to make recommendations on issues of national importance, including the quality assurance process, the national marketing and identification, and education and training strategies.

The operations group, as indicated on the chart, would carry out the overall coordinating function. Information would flow up from the regional, state, and local programs to this group, and it would administer the national quality assurance process. The operations group would review regional, state, and local programs wishing to be part of the national effort and would advise the ERM Board on whether the prospective programs met the national standard.

It is envisioned that the operations group would be a very small staff group who would suhcontract the majority of the work necessary to maintain the quality assurance process and undertake the appropriate information gathering function to various other organizations.

Regional, state, and local programs would function differently in different areas of the country. In some cases, an overseeing state body would exist to coordinate the operation of various local programs throughout the state. In other cases where no state organization existed, local approved programs would liaise directly with the operations group. Other scenarios would include situations where a state or regional program was the only program in existence covering a wide service territory.

The intent of the structure descrihed is to allow current or proposed programs with minor modifications to he part of the national effort, keeping the cost and effort associated with the overall national effort to a minimum, while affording the consistency necessary on a national hasis to satisfy the requirements of the mortgage industry. 


\section{Potential National Infrastructure}

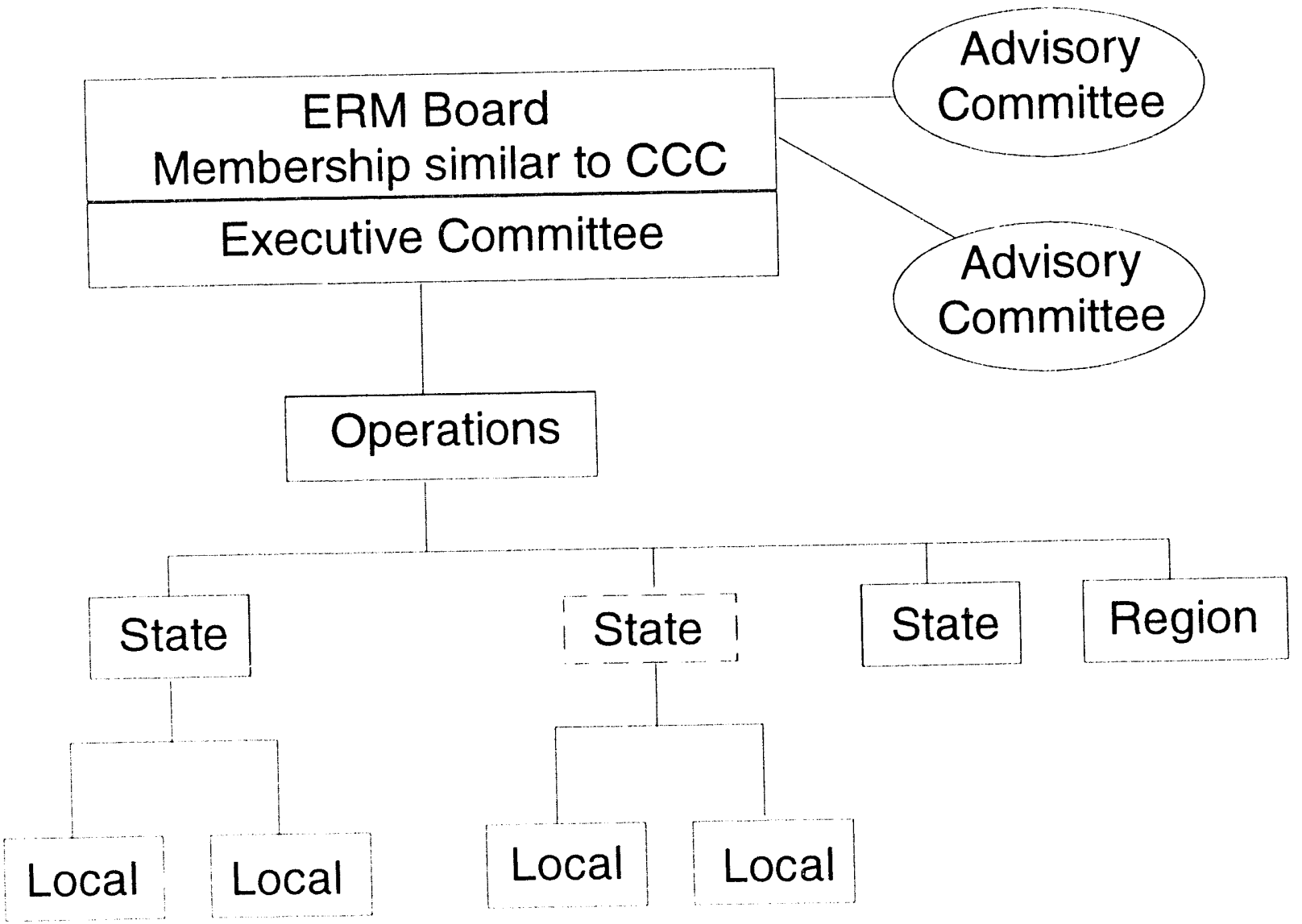


Issue Paper No.: $\quad 2$

Name of TAC:

Contact person: $\quad$ Frank Newbraugh, National Energy Specialists Association (Official Ohserver)

Date of issue paper: $\quad$ Septemher 10, 1991

Title of issue: $\quad$ Cost of alternatives (existing housing)

Recommendations (if any) (include majority/minority): Local contractor-based programs following a general national guideline would seem to he the most cost-efficient mechanism to deliver the HERS/EEM; need to run a model program or extensively monitor one of the few existing home programs.

Summary of analyses already completed:

LOCAL--.-.--NATIONAL

Programs range from municipal through state and regional to national implementation.

EXISTING HOME-...-...-...--NEW CONSTRUCTION

Programs range from existing home concentration through capability to complete neglect.

PRESCRIPTIVE

Programs range from highly prescriptive through special analysis to performance rating.

FEE FOR SERVICE--.............................

Programs range from full fee for service through subsidized to totally funded.

PRIVATE SECTOR-..-...-PUBLIC SECTOR

Programs range from small business through hig business to hig government.

\begin{tabular}{|c|c|c|c|c|c|}
\hline Actor & \#1 NESA & $\# 2 \mathrm{ERH}$ & \#3 UTILITY & 4 FNMA/HUD/FHA & \#5 R2000 \\
\hline Appraiser & (Entire & $\$ 50$ & $\$ 50$ & $\$ 50$ & (Entire \\
\hline Auditor & cost & $\$ 50$ & $\$ 100$ & (\#s hard to find, & $\operatorname{cost}$ \\
\hline Manager & horne & $\$ 100$ & $\$ 2(0)$ & so few johs done, & horne \\
\hline Specialist & by & $\$ 150$ & $\$ 2(x)$ & no "central" source) & hy \\
\hline Appraiser & contractor) & $\$ 50$ & $\$ 50$ & $\$ 5(1)$ & huilder) \\
\hline COST & $\$ 1000$ & $\$+() 0$ & $\$ 6(0)$ & $\$ 10(0)$ & $\$ 1500$ \\
\hline
\end{tabular}

Alternative solutions with advantages and disadvantages:

A. Costs: All programs require start-up cappital ranging from $\$ 25(1,()(1)$ per year for lecal, state, and

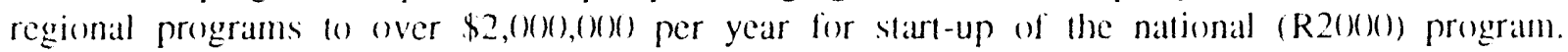
Additionally all programs, except \#1 and \#5, propose expenses for delivery of service on each home evaluated, an additional $\$ 40$ hillion on the least expensive option just 10 facilitate rating and improvement specifications $(1(x),()(x),()(x)$ homes times $\$ 4(x)$ equals $\$ 4(),()(x),(x)(),()(x))$. These expenses are to he horne hy the homeowner/huyer or hy government (the taxpayer). In program \# 
these expenses are all horne hy the contractor as a cost of generating husiness in existing home renovation, similarly $(0$ the expense heing horne hy the huilder participating in program $\# 5$.

B. Control: Programs \#2 and \#3 exercise good control over the actors at each step as the current program size allows, hut with expanded systems handling many more homes, there has heen concern expressed over loss of that control. Programs \#1 and \#5 place greal confidence in the honesty and capability of the contractor/builder and the ability of the guidelines to he achieved; hetter checks and balances need to he implemerited. Program \#4 has few actors and no controls.

C. Implementability: Programs \#2, \#3, and \#4 require the location, motivation, training, administration, etc. of large groups of diverse actors. According to reports given the Collaborative, these actors seldom have a good financial incentive to continue the work, and the public (the homeowners/buyers) doesn't feel the need to cover these additional costs. Programs that don't make good husiness sense are doomed to failure. Programs \#1 and \#5 require convincing husiness people that participation generates more return on their up-front costs, while eliminating the homeowner's out-of-pocket involvement. This is the way American business should work!

D. Financing options (not including start-up funding): Programs \#2, \#3, and \#4 place the burden of financing the rating and specification service (as well as location of contractors, realtors, lenders, etc.) on the homeowner/huyer; many of the programs are currently subsidized but the desire in all programs is to make them "self-funding." Programs \#1 and \#5 place all the expenses on the business person (contractor/builder) who expects to generate a profit from his work.

E. Johs: Programs \#3, \#4, and \#5 create few new johs; utility company personnel, realtors, lenders, appraisers, etc. are called on to provide more or slightly different services. Programs \#1 and \#2 require private small businesses to hire, train, and deploy vast numbers of new skilled employees, to generate both the ratings and to ultimately deliver the proposed improvements. Creating jobs generates the taxes to help defray the start-up expenses, which the cities and states should bear in these programs. Government should develop; husiness should deliver! 
Issue Paper No.:

Name of 'TAC:

Contact person:

Date of issue paper:

Title of issue:
3

Implementation

Richard Tracey, Ryland Homes

November 11, 1991

Cost of implementation-new construction

Recommendations (if any) (include majority/minority): An energy-efficient mortgage (EEM) program for new construction must be simple. A simple program would encourage widespread participation. Program elements could be:

- Homes built to the Model Energy Code (MEC) qualify for a stretch ratio.

- Homes built to standards higher then the MEC receive additional credit.

- A simple "certification of specification" he provided by a builder or designer.

- If the mortgage/hanking industry prefers dollar savings, data could be created that correlate the "certification of specifications" with energy dollars saved.

Organizations such as state energy offices, utilities, and local home builder's associations could conduct training for builders and designers. If a universal, simple format were developed at a national level (i.e., DOE or National Association of Home Builders [NAHB], Alliance to Save Energy) then the local agencies could conduct training at a nominal cost to builders and designers who want to be certified to complete the standard "certification of specifications." Recertification classes could be held yearly to deal with any updates in the requirements.

Startup costs would vary depending on the complexity of the program. Information on a "pass/fail" criteria based on the MEC is already available, and costs would be limited to distribution. The costs of organizing present data into the information necessary to correlate specifications with energy savings in dollars could probably be less than $\$ 250,000$.

A detailed discussion of program henefits and alternatives is presented as Appendix $A$ to this issue paper.

Summary of analyses already completed: Programs, such as the suburban Maryland and Virginia huilding industries E-7 programs, have demonstrated that huilders will respond to a simplistic approach and that costs can be kept to a few dollars per sale.

Numerous programs such as the NAHB Thermal Performance Guidelines, and the Department of Energy Voluntary Guidelines have compiled data to develop the value of incremental improvements for most geographic areas.

Research still needed (if any): Research is needed to establish the financial sensitivity of the results of prescriptive improvements. It would seem that if simplicity encouraged more huilders to participate, then the overall henefit to the nation's need to conserve energy would greally outweigh the few situations where upgrades did not perform to a precise measurement of savings. 


\section{Attachment to Issue Paper No. 3 Implementation TAC}

\section{Discussion}

An EEM program for new construction can be simple, and it could he administered at little or no cost. If the process was a simple verification of an accepted set of specifications, builders could upgrade present specifications with the assurance that additional financing is available if needed. If the process is complicated or a third party rating system is required, the costs and the time required to obtain a rating may discourage participation.

An opportunity exists to expedite a program, increasing the number of EEMs by setting the specilications at the Council of American Building Officials (CABO) Model Energy Code (MEC) level. Widespread adoption of the MEC has been very slow, and the approach could significantly increase voluntary upgrades to MEC standards.

The home-buying public has been paying the increased costs of construction for voluntary upgrades in efficiency with no universal assistance in financing those upgrades. This approach sets a recognizable baseline of specifications to assist buyers and builders who have voluntarily made the upgrades or for the few areas where home buyers already incur the cost of upgrades as a result of a local code.

Several options exist for the amount of mortgage adjustment.

- The current practice of "stretch ratios"

- Stretch ratios plus additional energy credits

- Energy credits only.

The simplest approach would recognize MEC specifications as a basis for awarding "stretch ratios." Additional efforts in conservation could be rewarded by calculating dollars saved over and above the MEC and applying direct credit to the housing expense (as proposed by the Association of Energy-Efficient Mortgage Service Companies).

If the mortgage banking industry wishes to deal only in dollars saved, a baseline lower than the MEC would need to be established, so that home buyers receive value for the costs involved in reaching the MEC level. Savings determined from that baseline could be represented as absolute dollars in the form of an energy credit to the housing expense.

The cost of implementation for recognizing the MEC as the basis for the stretch ratios could he limited to the distribution of the compliance manual being developed by CABO (with National Association of Home Builders [NAHB] and others) that translates the MEC requiremeits directly to specifications. A builder could provide a disclosure sheet to the buyer and to the lending institution that states the MEC requirement for his area and the specification for the huyer's home. This consumer could he protected by the normal means available to deal with fraud or misrepresentation if he or she has reason to believo that the specifications were not met.

The calculations to determine energy credits above the MEC or above any estahlished haseline would require a little more sophistication but they could he hased on a component approach similar to the average $U$ value $\left(U_{0}\right)$ requirement of the MEC. Data could be generated by local weather and hy local utility costs. That data could be made available in a series of charts that present estimated operating costs per square foot of each component, with modifiers for equipment efficiencies. A huilder or designer 


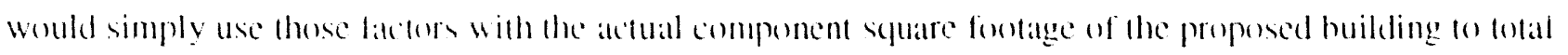
energy savings.

Ohviously, a compuleried version could he crealled. If both the hand format and the compuler printout are standardiced, the morgage/hanhing industry would have the information it needed.

Most of the data needed to generate this type of report alleady exist in the work done to crealle DOE's voluntary energy standards and the NAHB Thermal Performance Guidelines. This data could he used as a hasis for the creation of the necessary hand or computer generated report.

The key to success will be simplicity. Other fools may he used to evaluate more complex siluations such as passive solar huildings. However, those tools should provide reports in the same format as the "certification of specifications" correlation report, and the immediate availability of such tools need not he a criteria for adoption of the hasic program.

Novemher 11,1991 
Issue Paper No.: $\quad 4$

Name of 'TAC:

Contact person: $\quad$ Becky Cohen, Consumer Federation of America

Date of issue paper: $\quad$ November 5,1991

Title of issue: $\quad$ Consumer protection aspects of HERS/EEMs

Recommendations (if any) (include majority/minority):

1. Participation in the national system shall he open to all HERS/EEMs programs that meet the standard criteria developed hy a consensus of affected parties. The national criteria shall encompass HERS and EEM programs for both new construction and existing residences of all types.

2. To be nationally recognized, a HERS/EEM system shall have huilt-in fatures designed to prevent consumer problems. These features shall include:

- Clear, simple, accurate, comprehensive, and unbiased consumer education materials and point of purchase disclosures about hoth HERS and EEMs. These shall he consistent from one program to another and contain disclaimers, as necessary, so as not to rilislead;

- An energy rating system and technique that is technically credible and that includes:

- a credentialing system for raters

- a validation scheme for ratings

- a systemwide cuality assurance program

- ratings and labels that are consistent from one jurisdiction to another and enable market comparisons within a jurisdiction;

- Timely disclosures about the availahility of EEMs to every mortgage applicant.

Research still needed (if any): Caltalogue consumer protection features of existing HERS/EEM programs and any consumer protection problems they have encountered. 
Issue Paper No.:

Name of TAC:

Contact person:

Date of issue paper:

Title of issue:
Implementation

Becky Cohen, Consumer Federation of Americal

November 5,1991

Resolution of consumer problems associated with HERS/EEM

\section{Recommendations (if any) (include majority/minority):}

1. To the extent possible, a national HERS/EEMs system shall utilize the resources already available in existing institutions that educate and inform consumers, respond 10 consumer incjuiries and complaints, mediate consumer disputes, and regulate service providers.

2. As part of its quality assurance program, the national HERS/EEM coordinating entity shall have the capacity to periodically collect data from participating programs and from consumer protection institutions in order to identify trends and any problems or program design features that warrant modification.

Summary of analyses already completed: To enlist their cooperation and to make them effective, a national HERS/EEM program will provide these institutions with training and reference materials to prepare them to offer consumer protection services in connection with HERS/EEMs questions, problems, or disputes.

For example, the national coordinating entity might commission guides that explain the program and specif the criteria nationally recognized or certified HERS/EEM systems must meet. Desk references would be useful for consumer protection agencies and institutions whose employees will be expected to respond to questions and complaints in the field.

Data from consumer inquiries and complaints can be extremely useful for troubleshooting and for longer term program oversight and quality control. Data indicating that there are few or no complaints would be informative; the data reporting system must be in place in order to learn that there are no problems to report.

Research still needed (if any): Identify existing public and private consumer protection institutions that should he included in HERS/EEM implementation, and identify any major shortages in consumer protection services that the Collaborative may want to take steps to fill. 
Issue Paper No.:

Name of 'TAC:

Contact person:

Date of issue paper:

Title of issue:
6

Implementation

Benny Folsom, Southern Electric International, Good Cents Division

September 20, 1991

What are liability issues and options"?

Recommendations (if any) (include majority/minority): The issue of liability should be considered. A hasic understanding is necessary for planning and implementing a HERS system that provides proper training and qualifying of "raters" and, therefore, limits the opportunities for negligence, misrepresentation, and consequent damages that might inhibit the acceptance of the HERS hy its heneficiaries.

Summary of analyses already completed: If HERS systems are to provide a beneficial service to their users (home huyers, home builders, realtors, lenders, appraisers, etc.), unfounded concerns that could be associated with liability need to be addressed and understood; otherwise, the fear of potential liability may decrease the overall effectiveness of the HERS and reduce the value of information available to the group of users. Potential liability should be viewed as a legitimate concern; however, careful consideration of hoth the intent and legal positioning of the HERS should keep liability from hecoming an overriding concern. Across the nation, disclosure of a home's energy efficiency status has become a commonplace occurrence by programs sponsored hy state and private organizations. To date, legal precedents are very rare where a homeowner or rating system user alleges that a rating system was negligently designed, applied, or performed (that resulted in consequential damages) even though existing systems have seen widespread usage. Aside from the implementation of a technically sound and professionally administered rating system, the next most important and effective consideration is full disclosure of the rating system's working assumptions and limitations and caveats covering areas not implied or verified (such as building codes, data sources, or rating system output disclosures). It is generally believed that the incorporation of broad disclaimers could compromise the goal of consumer protection if it raised serious questions about whether the rating is meaningful. These disclosures should not be put in fine print that could easily be overlooked or ignored. Disclosure should assist the successful incorporation of energy efficiency into market value and further encourage adoption of the energy conservation ethic.

The general sense is that liability has not had a significant negative impact on home energy systems in the past and should not be allowed to become a negative issue in the implementation of a HERS/EEM system.

Research still needed (if any): Review of legal records involving HERS for information on attribution of damages due to negligence, malpractice, or invasion of privacy claims; investigate the possibility of providing malpractice insurance coverage (for the rater, sponsor, developer, etc.). Incorporate experience of practicing HERS programs, to the extent that is applicable.

\section{Bibliography:}

P.L. Henurickson, Liability Aspects of Home Energy Rating Systems, 1983, Pacific Northwest Laboratory; Richland, Washington. 
Issue Paper No.:

Name of TAC:

Contact person:

Date of issue paper:

Title of issue:
7

Implementation

Doug Seiter, City of Austin

November 5, 1991

Human resource needs

Recommendations (if any) (include majority/minority): No recommendation is given at this time. When the specifics of a national energy-efficient mortgage (EEM)/home energy rating program are determined, resource needs, including human and financial resources, should be published. Most helpful would be a "how to" manual for program startup and implementation.

Summary of analyses already completed: Staffing requirements are as varied as the number of programs currently working. The needs are relative to the complexity of the rating tool (computer or worksheet), the information requirements of the tool (from plans or site inspection), the type of home rated (new or resale), the technical expertise required of raters, and the level of quality control (site inspections) required for program integrity. Several working programs were analyzed for resource needs.

While there are significant variations in currently operating home energy rating systems, there is some consistency that begins to appear in the fundamental aspects of program operation. For the sake of comparison, time required for the following functions was estimated for four existing programs:

1. Initial site analysis (built home)

2. Takeoffs from plans (new construction)

3. Calculation/computer analysis

4. Site inspection after rating

5. Transportation

6. General administration (scheduling, processing, and distributing rating and billing).

Program A, Municipal, Sliding Scale Rating

Total time per rating: 4-9 hours

Initial data collection, whether it is from plans or site visit, varies from 1-4 hours, depending on the size and complexity of the house. Preparing for and completing the computer analysis may take another $2-4$ hours. Overhead time to include transportation, scheduling, preparing, delivering the output report, and billing would add about another hour to the time. Approximately 350 ratings have been done since program implementation in 1989. Ratings are done by independent contractors, and the cost of the rating is currently being subsidized by the utility, with a continuation in the subsidy being considered. The program is currently being run by one full time manager.

Program B, Utility, Certification

Total time per rating: $7-12$ hours

Initial data collection from plans and running the computer analysis takes from 1-3 hours. Three inspections take place during construction, which average $1 / 2$ hour each, not including transportation. Transportation, because of the large service area, may add 3-6 hours. Administration time to deliver the certification and process the rebate check that accompanies the certification may add another $1-1 / 2$ hours. A goal of 350 certifications has been set for this year. One full time manager runs the program, with part time administrative assistance. Field personnel for conducting inspections also assist in other utility programs. 
Calculation, including data collection from plans or field notes, is about 2 hours. Preparation of the report adds another hour; and $1 / 2$ hour covers administrative time, sans scheduling (scheduling is done by field representatives). Site inspection, either for initial data collection or follow-up, requires 1-4 hours. A minimum of three staff (director, administrative, computer) is required for program operation, in addition to field rating staff (private contractors), which varies according to volume.

Program D, Municipal, Sliding Scale Rating

Total time per rating: $1-9$ hours

New homes are rated from plans; there is periodic consultation with home buyers preparing to build. Time varies widely according to complexity of the home, from $1 / 4-8$ hours. Entry of data and running of the computer analysis takes 5-10 minutes. Another $1 / 2$ hour is figured as an average per rating for builder contacts and various marketing functions, including one-on-one presentations, which are seen as essential to program success. Preparation of the report and marketing materials is minimal. Inspections for alternate compliance to the energy code averages $1 / 2$ hour per rating. Two rating staff and a program manager produce about 600 ratings per year. Field inspections are done by department field personnel who also work with other programs.

Alternative Solutions:

Contractor/builder performs and distributes rating:

- Contractor/builder in the best position to provide rating information.

- Cost of rating may be absorbed as marketing costs.

- Could have access to "approved" rating tools through national network.

- Liability rests on each contractor/builder to provide accurate information.

- Tool must be very user friendly.

- Credibility and conflict of interest will be issues.

Owner/buyer performs rating:

- Technical limitations even more severe than with contractor/builder; tool must be so simple that it may compromise reasonable accuracy.

- Positive educational value.

- Limited or no liability.

- Low/no cost.

- Not likely to meet EEM requirements.

Electronic rating through home computer/cable TV:

- May work well as a national rating system.

- Development of software coincides with advances in home electronics.

- Could be localized in energy analysis programming.

- May require significant capital, limiting local development.

- Could climinate current programs.

- Revenue could be generated through "pay for use." At a reasonable cost, multiple use of the rating would occur, particularly if users could "play" with different options to increase ratings.

- Must he very user friendly. Wide variation in technical ahility.

- Applicability to EEM questionahle.

Research still needed (if any): Organization and structure of the national program. 
Issue Paper No.: $\quad 8$

Name of TAC: $\quad$ Implementation

Contact person: $\quad$ Chris Roitsch, National Association of State Energy Officials

Date of issue paper: $\quad$ November 12,1991

Title of issue: $\quad$ States' role/incentives for participation

Recommendations (if any) (include majority/minority): A state-level agency is needed to coordinate with state-level stakeholders in developing strategies for implementing a voluntary HERS/EEM system. State energy offices should serve in this role.

Technical and financial incentives are necessary for states to participate in the national program.

\section{Summary of analyses already completed:}

Incentives for State Participation: Incentives necessary to encourage participation may differ somewhat from state to state, depending upon the presence of existing programs, the need to bring them into uniformity, the coverage of existing programs (statewide or regional), how well existing programs are performing, industry's support or resistance, and public awareness of benefits.

To encourage their participation, states need a system that:

1. Provides a complete package of tools, including: (a) approved rating models, (b) rating tools, (c) quality control processes, (d) training materials, and (e) promotional/marketing tools.

2. Offers a real potential to increase the energy efficiency of housing stock. This implies a clear linkage of a rating system to an energy-efficient mortgage. While several states have or are developing home energy rating systems, few utilize an energy-efficient mortgage. An effective linkage that leverages funds for energy improvements would serve as an incentive for states to participate.

3. Is attractive and easy for the consumer to utilize.

4. Requires minimal resources to develop and implement.

5. Is flexible enough to allow present programs to be incorporated.

6. Provides financial resources to assist implementation. While states may fully recognize the value of a HERS/EEM system, few will have adequate state financial resources to implement one. This is especially true of the heavy front-end start-up costs. National-level funding is necessary to bridge the transition from start-up until such time as fees, public-private partnerships, or other funding sources begin covering ongoing implementation costs.

7. Provides ongoing technical and promotional support from a national clearinghouse.

States' Potential Roles: Many factors will influence the role a state chooses to play in implementation. These include the features of the collaborative's final product, the needs and conditions of the state's shelter industry and consumers, available resources, etc. States will choose different delivery mechanisms-utilities, state agencies, industry groups, or nonprofit organizations. Working through various state agencies and stakeholders, state and local funding sources can be identified. Potential roles for states in implementing a HERS/EEM system are: planning, leadership/coordination; coordination with national organizations, training and technical assistance; quality control and monitoring; consumer awareness; evaluation; data collection; certification; and program operation. These roles are further defined in the attachment to this paper. 


\title{
Attachment to Issue Paper No. 8 \\ States Roles/Incentives for Participation
}

\begin{abstract}
State Energy Offices
States routinely set policy for and then plan and implement a broad range of energy and housing initiatives. In 1973, state energy offices (SEO) began planning and implementing energy programs in response to federal statutes designed to mitigate the effects of energy shortages. Since then, the energy supply situation has changed, and emphasis has shifted to more efficient use of available resources. Throughout this evolution, SEOs have cooperated closely with the Department of Energy to utilize hoth state and federal resources to achieve specific energy goals. For example, SEOs have actively worked to establish building energy efficiency standards as required under the State Energy Conservation Program.
\end{abstract}

\section{Survey of States}

The National Association of State Energy Officials conducted a survey of states in February 1991 to determine their interest and involvement in home energy rating systems. Forty-six states participated in the survey. The survey found:

- 12 states already have a home energy rating system in operation

- Of the states that have systems, 7 utilize energy-efficient mortgages

- 17 states are in the process of developing a home energy rating system for their state

- 12 others are interested in having a home energy rating system

- For states developing rating systems, the main barriers are lack of resources, need for information, and concern for the accuracy of the system.

\section{$\underline{\text { States' Potential Roles }}$}

Planning - Serve as central coordinator for developing a state plan for implementation. Secure input from state-level stakeholders on strategies suited to the particular needs, conditions, and resources of the state.

Leadership/State Coordination - Secure the support of state and local officials and other agencies with technical or financial resources to support the HERS/EEM system. For example, state housing agencies and public utility commissions could be instrumental in encouraging financial support through utilities and local housing organizations. Coordinate ongoing communication among stakeholders to avoid duplication, share experiences, and enhance quality of implementation.

Coordination with National Organization, DOE, HUD, Other States - Serve as the central point of contact with the national clearinghouse and federal agencies. Share experiences with other states to improve implementation nationally. 
Training and Technical Assistance - Utilize existing expertise to develop and distribute training and technical assistance materials and tools. Conduct or coordinate training for raters, builders, real estate professionals, appraisers, and others. Have staff available for technical assistance to stakeholders as implementation progresses.

Quality Control and Monitoring - Develop systems to monitor implementation and recommend modifications as needed. Feed information gathered back into the training and technical assistance function.

Consumer A wareness - Incorporate into existing consumer education networks and programs such as the Energy Extension Service Program, or develop specialized consumer awareness efforts tailored to the national HERS/EEM system. Coordinate with stakeholders to maximize individual marketing activities.

Evaluation - Evaluate program impacts and gather information for feedback to marketing activities.

Data Collection - Collect information at the state level to feed into the state and national data bases.

Certification - States act as oversite to assure that materials and tools are consistent with state objectives and national guidelines. State certification is necessary to enhance the credibility of the system for local stakeholders and consumers.

Program Operation - Deliver services in areas not served by other jurisdictions. 
Issue Paper No.:

Name of TAC:

Contact person:

Date of issue paper:

Title of issue:
Implementation

Russell Duncan, Carolina Power and Light

October 11, 1991

Would incentives be needed to encourage local participation in a national system or program; and, if so, what form would they take?

Recommendations (if any) (include majority/minority): Education/communication by states, localitics, federal agencies, builders, realtors, utilities, and lending agencies on the value of energy-efficient homes will provide some incentive for home buyers. The key is to provide a demand by offering incentives that influence home buyers to purchase higher efficiency structures. Existing incentives include billing credits by utilities; energy savings related to better built homes; direct rebates to the buyer, builder, and/or realtor; and other incentives. A combination of these, plus lending agency credits/incentives, will pull the market toward higher efficiency. For example, lowering the interest rate (on the most energy-efficient houses) by $1 / 8 \%$ to $1 / 4 \%$ would create an additional/real incentive for building efficiently. Influencing the buyer will, in turn, influence all other players to respond to buyer demand and a move toward higher efficiency.

Summary of analyses already completed: A number of studies have been conducted to determine the value of incentives. It is generally agreed that incentives are needed to "jump start" the market and sustain it. A relatively small number of participants will be attracted with no incentives. That number will increase with the level and number of incentives. For example, the utility company may provide special billing discounts and direct rebates (to buyers, builders, and realtors) while lending institutions allow lower ( $1 / 8 \%$ to $1 / 4 \%$ ) borrowing rates and/or a higher ceiling on the amount loaned. Federal incentives (tax credits) could be applied to homeowners who build this energy-efficient home. If we want to create market demand, then each segment-lender, borrower, builder, realtors, utilities, and federal, state, and local agency-should offer an incentive. The program may work without incentives; however, it is unlikely that significant initial or sustained long-term participation would result without them.

Research still needed (if any): Survey the lender, builder, buyer, realtor, utility, and state, local, and federal agencies to determine what additional incentives they are willing to offer. 
Issue Paper No.:

Name of TAC:

Contact person:

Date of issue paper:

Title of issue:
10

Implementation

Barry Moline, American Public Power Association

October 31, 1991

What are the actions necessary for quality control, who would carry them out, and what would be the estimated costs?

\section{Recommendations (if any) (include majority/minority):}

1. Each organization sponsoring the home energy rating system (HERS), be it state, utility, local government, or private organization, should be responsible for its own quality control in accordance with the quality control guidelines of the national HERS organization.

2. A quality control plan should be a requirement of the certification process with a national HERS organization.

3. Costs for quality control should he borne by the organization sponsoring the home energy rating system.

Summary of analyses already completed: Quality control is a function that must be conducted within each state, utility, or locally controlled HERS structure. Every HERS should have a quality control plan that sets out the procedures that will be used under the system. Components of the quality control function include:

- Training and certification of raters;

- Random audits of raters;

- Periodic recertification of raters;

- Archiving of ratings; and

- Validation of ratings against actual energy consumption.

There are several ways to conduct these activities. First, HERS should have a training program whereby new raters can be certified and existing raters can be periodically retrained and recertified. Second, to satisfy the requirement of lending organizations that energy expenditures be in-line with predicted values, ongoing evaluation must be conducted to show that predicted and actual expenses for each house correlate. Utility meter data should be used, corrected for factors that would alter the relationship between predicted and actual values, such as weather, energy improvements, and home expansion. Finally, these data can be used to rate the raters and determine, on an individual basis, if any are conducting ratings that are incongruous with reality. This evaluation could also serve as a check on the validity of the building energy predictive model.

The costs for quality control should be horne hy the organization sponsoring the home energy rating system. These costs can be assumed internally or can be contracted out. For example, archiving of ratings may be done within each HERS, on a statewide basis, or with a national organization. Costs will vary by type, as well as the amount of staff time required. 
Issue Paper No.:

Name of TAC: $\quad$ Implementation

Contact person: $\quad$ Barry Moline, American Public Power Association

Date of issue paper: $\quad$ Octobır 31, 1991

Title of issue:

Who should verify the adequacy/accuracy of building energy predictive tools?

Recommendations (if any) (include majority/minority):

1. Organize a HERS industry organization based at the offices of one of the following:
a. Office of Building Technologies at DOE;
b. Nonprofit, independent energy research organization;
c. HERS industry association; or
d. Some combination of the above.

2. Groups with HERS that want to obtain certification of their programs from the national organization would be required to make application to it.

This question begs a broader issue: the need for a HERS industrywide organization to conduct, among other things, verification of building energy predictive tools. This organization could be tasked with certifying each HERS in accordance with national guidelines. By certifying HERS, the national organization would set the standard (based on the results of the collaborative) by which all HERS must qualify, thus setting a common denominator for all programs. The organization would be housed at either the DOE Office of Building Technologies, a nonprofit, independent energy research organization, HERS industry association, or some combination of all. It could be the role of a national laboratory to develop test procedures (as identified in Issue Paper No. 5) to evaluate the building energy predictive tools that would be implemented by the national organization.

A process should be developed by which a HERS would be certified and predictive tools analyzed. Application could be made to the certifying organization, with justification provided for each criterion. It may be necessary to provide engineering documentation for the energy predictive model, as well as conduct a demonstration. 
Issue Paper No.:

Name of TAC:

Contact person:

Date of issue paper:

Title of issue:
12

Implementation

Carson D. Culbreth, National Association of State Energy Officials

November 6, 1991

What aspects of a combined HERS/EEM program might be mandatory?

Recommendations (if any) (include majority/minority): Participation by the consumer in a HERS/EEM program shall be voluntary. Participation by authorized jurisdictions in HERS/EEMs should remain voluntary; however, upon election to participate, the jurisdiction shall adhere to a detailed listing of mandatory guidelines. These guidelines should include, at a minimum, the following:

1. One performance-based, 0-100 rating scale for each jurisdiction based upon climate and construction practices.

2. Standard threshold point on the rating scale, which establishes the minimum efficiency level for HUD, VA, FmHA, Fannie Mae, Freddie Mac, and Ginnie May insured or guaranteed EEMs.

3. Minimum documentation requirements meeting audit requirements of HUD, VA, FmHA, Fannie Mae, Freddie Mac, and Ginnie Mae.

4. Standard reference source for establishing energy consumption by climatic zone and structural type for comparison against predicted consumption that will be used in the EEM process.

5. Standard procedure for testing and certifying the accuracy of rating tools by impartial third party. Where computer simulations are used for the certification, the specific program, such as DOE-II, should be identified.

6. Standard labeling for homes receiving ratings.

7. Standard minimum training requirements for individuals performing ratings.

8. Standard procedures for monitoring, quality control, and evaluation. These procedures shall be implemented by an impartial body other than the rating organization.

9. Procedures for reporting on rated houses to assess impact of program through upgrade of existing housing stock, issuance of EEMs, and default of EEMs compared to conventional mortgages.

10. Procedures to ensure the consumer will be advised of the availability of EEMs.

11. See research.

\section{Alternative solutions with advantages and disadvantages:}

\section{Advantages}

1. National consistency in the evaluation and implementation of HERS/EEM initiatives.

2. Developmental costs at state and local level are reduced.

3. Allows local autonony in the development and delivery of individuai programs, including implementation as an integrated resource planning (IRP) initiative.

4. Provides consistent hasis for underwriting mortgages.

5. Allows incorporation of local code requirements as point on scale with quality control standards reinforcing code review.

6. Assures impartial third party validation of both rating systems and homes rated. 


\section{Disadvantages}

Local autonomy in development and delivery may permit abuses, compromising integrity of program.

\section{Research still needed (if any):}

1. Revicw of IRP for potential implementation of HERS/EEM prot ms.

2. It is assumed the collahorative will design a program that will assure full participation hy all key governmental financial institutions. In the event that the governmental financial institutions do not voluntarily participate, research should be conducted to determine the feasibility of mandating their participation. 
Issue Paper No.:

Name of TAC:

Contact person:

Date of issue paper:

Title of issue:
13

Implementation

Javier H. Figueroa, Washington State Energy Office

October 31, 1991

What are the roles of the various participants in avoiding delay in the loan approval process?

Recommendations (if any) (include majority/minority): A strong, ongoing education for consumers needs to be developed and implemented in order for energy-efficient mortgages (EEMs) to be demanded. Alternatively, the buyer can request to take advantage of the EEM program. A similar program, like Federal National Mortgage Association's Community Home Buyers Program, should be looked at, whereas, the borrower is required to attend a home buyer's workshop in order to obtain a favorable low down payment loan.

The key to success is having the lead organizations that have a fundamental stake in the success of the program (real estate and lending) committed and motivated. The real estate industry would need to take a major step, requiring borrowers to pre-qualify prior to showing the customer any homes. The lending industry, in cooperation with the real estate industry, would incorporate a home buyer's workshop as part of their pre-qualification. Thus, the consumer can be educated on the EEM program and can decide to take advantage of the program at that point. This approach would help eliminate any delay normally associated in the loan approval process.

The secondary market can assist in removing red tape by setting uniform, streamlined underwriting rules for EEMs; they can also establish a uniform appraisal/valuation method for EEMs and permit alternatives to escrow arrangements. Lenders are adverse to creating escrow accounts at closing because they create complications with the transaction and mortgage pipeline.

\section{Summary of analyses already completed:}

1. Community Home Buyers Program.

2. Mortgage pipeline.

3. A policy paper for the Alliance to Save Energy Conference (by Perry Bigelow).

4. Home Energy, Nov./Dec. 1988.

5. Implementing HERS, Lawrence Berkeley Laboratory, Berkeley, California.

6. Real Estate Finance Today (7/28/89).

\section{Research still needed (if any):}

1. New appraisal/valuation method needed.

2. What incentives would be required to motivate the real estate and lending industry?

3. Find a third party for dispersing escrow funds for energy improvements.

4. What information should be incorporated in a home buyer's workshop? 
Issue Paper No.:

Name of TAC: $\quad$ Awareness

Contact person: $\quad$ Rebecca Vories, Infinite Energy

Date of issue paper: October 31,1991

Title of issue: Generation and distribution of HERS/EEM information for consumers and shelter industry audiences

\section{Statement of Issue:}

There are a number of inextricably related issues in increasing awareness regarding ratings of houses and availability of special lending arrangements for energy-efficient or efficiency improved homes. Some of these issues have to do with the type and depth of information that needs to be made available to consumer or industry audiences and others have to do with who generates that information and how it is distributed. In either case, the specifics that are to be promoted have a great deal of impact on the information developed and the best means of creating and disseminating that information. Most of the issue papers assigned to this committee have dealt with the type and depth of information that may be needed. This paper deals with who generates and disseminates the information.

As we see it, there are five possible models for who generates and disseminates information.

Model A-A single, national awareness effort that is created by a single organization and develops and disseminates all the information, education, and training.

Model B-A primarily national awareness effort where some centralized group generates some generic information that is then turned over to each national participating organization, which at a minimum passes the information along to its membership and at a maximum expands the information into extensive training and education efforts.

Model C-A primarily program operator effort (e.g., individual state, utility programs) where some centralized national group generates information about common agreements that individual program operators incorporate into their own awareness efforts.

Model D-A primarily certification effort where the primary communication is to those organizations that want to operate a certified program and explains what they will need to do in order to be certified as a participant in the national program.

Model E-A national clearinghouse that gathers all information about existing and new programs, and makes it available to interested parties along with summary information based on information gathered from other sources. (Awareness TAC Issue Paper No. 5 describes this model in some detail.)

Recommendations (if any) (include majority/minority): At this time, we do not recommend any of these models more than any other.

Summary of analyses already completed: We cannot go beyond the most generic observations about awareness needs until we know more about what a "national" HERS/EEM program will look like and who will be responsible for "operating" it. There has been no attempt to identify awareness program models in other arenas that might be good models from which to learn. We are not sure if any exist or not. 
Alternative solutions with advantages and disadvantages:

Below is a display of the advantages/disadvantages of each model. These models are not mutually exclusive but some are more likely the.n others to be the "lead" model.

\begin{tabular}{|c|c|c|}
\hline Mode! & Advanrages & Disadvantages \\
\hline Model A & $\begin{array}{l}\text { Consistent messages about all issues to all } \\
\text { audiences and delivered based on some } \\
\text { perceived priority basis. }\end{array}$ & Highly unlikely to take place. \\
\hline Model B & $\begin{array}{l}\text { The national organization responsible for } \\
\text { each aspect of this industry can best } \\
\text { communicate in the language of its } \\
\text { constituericy and would be the most } \\
\text { credible to its industry and has already } \\
\text { developed communications channels that } \\
\text { can be tapped. }\end{array}$ & $\begin{array}{l}\text { Possible inconsistency of } \\
\text { messages. Not all organizations } \\
\text { will devote required level of } \\
\text { effort to fully educate their } \\
\text { membership in the option time } \\
\text { frame or help them educate } \\
\text { consumers. }\end{array}$ \\
\hline Model C & $\begin{array}{l}\text { Each program operator has the greatest } \\
\text { motivation to make their program } \\
\text { successful and draw in all those local } \\
\text { elements that are critical to its success. } \\
\text { Nationally generated information would } \\
\text { be tailored to dovetail with the specifics } \\
\text { of each program. }\end{array}$ & $\begin{array}{l}\text { Possible inconsistency of } \\
\text { messages. Not all program } \\
\text { operators have involved or been } \\
\text { able to involve all necessary } \\
\text { industry participants. All } \\
\text { program operators have sufficient } \\
\text { funding to carry out an adequate } \\
\text { awareness effort. }\end{array}$ \\
\hline Model D & $\begin{array}{l}\text { This would be the most focused effort } \\
\text { and would be primarily aimed at } \\
\text { recruiting current and future programs to } \\
\text { be certified and to operate under certified } \\
\text { conditions, which might include certain } \\
\text { awareness activities. }\end{array}$ & $\begin{array}{l}\text { Uncertainty about what } \\
\text { organization would be the } \\
\text { certifying agency, what would be } \\
\text { certified, and how implemen- } \\
\text { tation would be monitored. }\end{array}$ \\
\hline Model E & $\begin{array}{l}\text { Provides a central source of much } \\
\text { valuable information. }\end{array}$ & $\begin{array}{l}\text { Is a passive rather than an active } \\
\text { role vis-à-vis awareness creation. } \\
\text { Probably better serves program } \\
\text { operators than consumers. }\end{array}$ \\
\hline
\end{tabular}

Research still needed (if any): Primarily, we are awaiting decisions about what the "national" program looks like. Once those decisions are made, along with some sense of the types of cash and in-kind resources that will be available to support awareness activities, we can make more specific recommendations about which information generation and dissemination model seems to hest fit the type and depth of information that will he required hy both consumers and industry audiences for such a program. 
Issue Paper No.: $\quad 3$

Name of TAC: $\quad$ Awareness

Contact person: Deborah Howard, Arizona Energy Office

Date of issue paper: October 21, 1991

Title of issue: What are the motivational and technical needs of the following groups (see summary section for groups)?

Recommendations (if any) (include majority/minority): Create an awareness campaign that includes the internal and external motivational factors and technical needs of the stakeholders as described below.

Summary of analyses already completed:

Mortgage Lenders: Motivational - Need simplicity in documentation, expanded profit opportunities, diminished risk, and federal, state and local sponsored promotions.

Technical - Need accurate and understandable training.

Appraisers: Motivational - HERS/EEMs will provide expanded professional expertise to now include energy. Increased house sales makes for increased profits. Increased home sales with information allows development of comparables.

Technical - Need accurate training on added value of energy-efficient items. Need ongoing technical assistance.

Iuspectors: Motivational - HERS/EEMs will provide expanded profersional expertise.

Technical - Need detailed technical training and product information.

Real Estate Agents: Motivational - Offers expanded professional expertise. Will provide increased profits through sales and sales contests, commission incentives for brokering EEMs, federal/state funded promotions, and customer requests.

Technical - Need accurate and understandable information on HERS, detailed mortgage information.

Code Officiais: Motivational - Need funding to support increased staff for inspections and reporting.

Technical - Need detailed technical training on HERS and how to use in code compliance.

Builders: Motivational - Offers competitive edge in marketplace. Enhances public image. Need high visibility with minimal cost. Allows participation in utility rebate program and EEMs. Cost benefit analysis. Federally sponsored promotional campaign. Develop partnerships with utilities, industry.

Technical - Need accurate information and training on HERS, design assistance.

Remodelers: Motivational - HERS/EEMs enhance public image, competitive edge in marketplace, and expanded professional expertise. 
Technical - Need training and accurate information on cost-effectiveness of energy measures and best construction techniques.

Manufactured Housing: Same as builders.

Product Suppliers: Motivational - HERS/EEMs offer opportunity for new product and program development. Marketing tool to sell the energy efficiency of product. Marketplace will dictate need for increased sales.

Technical - Need accurate information on product's energy efficiency. Need training on flexibility of program to model new product.

Consumer Groups: Motivational - HERS/EEMs offer self-motivating information on advocacy financial benefits, added comfort, lower utility bills, lessened environmental degradation, wise use of existing resources (indirect cost of energy use), clearinghouse with information on certification/monitoring.

Technical - Need training for advocacy support of programs.

Utilities: Motivational - HERS/EEMs enhance image and can generate internal motivation by helping people see concrete results (by interpreting bills, usage), a tool for least cost planning and demand-side management programs. Need cooperative programs for promotion. External incentives include discount coupons for products and utility rebates to builder/homeowner.

Technical - Need detailed training on HERS/EEMs and continued technical assistance if performing the ratings. Third party information for rate cases.

Regulators: Motivational - HERS/EEMs implementation mechanism for least cost planning (lowest possible rates) and environmental benefits of program.

Technical - Need accurate information for development or analysis of least cost planning programs.

Relocation Companies: Same as realtors.

Homeowner Associations: Motivational - HERS/EEMs offer opportunities to upgrade neighborhood, home comparisons, and lower cost of homeownership.

Technical - Need general information on programs and retrofit possibilities.

State and Local Governments and Legislative Bodies: Motivational - Need national media campaign to run concurrently with state/local activities and national and private sector funding support. HERS/EEMs enhance image to public and constituencies and offer leadership in energy conservation and economic development and savings in dollars and environmental costs.

Technical - Need accurate information and training for program development, oversight, and certification and codes.

Product Sunpliers and Manufacturers: Need information on how their product can work and/or he certified and henefit the program.

Organized Consumer and Environmental Advocady Grouns: Need ow he directly involved in information programming. Need information on saving possihilitiss and list of participating lenders/builders. Nee! 
details of monitoring of program and information for future legislation planning and codes. Need for energy savings and value on social environmental externalities.

Utilities: Need accurate information to develop demand-side management programs that meet their needs and tie in to their other residential real estate programs. Need similar promotional materials as noted in Awareness Issue Paper No. 4.

Regulators: Need accurate and understandable information for development and evaluation of least cost planning programs and effective implementation of specific programs.

Relocation Companies: Same as realtors.

Real Estate Attorneys: Need accurate information and documentation for sales contract review.

Homeowner Associations: Need accurate information on benefits to the buyer/seller. Need information on retrofit products and impact on the covenants and restrictions in their area.

State and local executives and legislative bodies: Need updates on progress of program for future funding, programming support, legislation, and benefits to their constituents.

\section{Research still needed (if any):}

1. A model for implementation and promotion must be designed to meet the informational needs of the stakeholders.

2. Meet with the stakeholders/groups when there is concurrence on the HERS/EEM tools/program guidelines to further investigate communication and promotion mechanisms. 
Issue Paper No.: $\quad 4$

Name of TAC: $\quad$ Awareness

Contact person: $\quad$ Gerry McGowan, U.S. Department of Energy Philadelphia Support Office

Date of issue paper: Octoher 9, 1991

Title of issue: What are the information and education needs of consumers and how can they best be met?

Recommendations (if any) (include majority/minority): Consumers need specific, relevant, graphic, and credible information that relates the benefits of HERS/EEMs to their immediate home buying, selling, and remodeling needs.

Some of the tools to accomplish both consumer information and education include:

- TV and radio spots

- Newspaper ads

- Brochures

- Press conferences and newspaper columns

- Tours, media events, home shows

- Labels

- Central clearinghouse

- Personal contacts.

The most effective means of convincing consumers to participate in HERS/EEMs is a credible source. Specific messages will need to be tailored for buyers vs. sellers and for homes that are already efficient vs. those that need to be brought up to a more efficient level.

It is recommended that a centralized clearinghouse, accessible through a toll free number, be established. The clearinghouse should be the central contact point advertised in mass media campaigns. The central clearinghouse would supply local contact points for specific HERS/EEMs information. Local contact points would include participating realtors, bankers, utilities, home builders, and home remodelers.

DOE's Regional Support Offices and State Energy Office should also be included in this network of local contacts.

It is further recommended that the participating memhership of the Collaburative provide the leadership and direction to its local member organizations, so that their participation in HERS/EEMs consum ir education will he relevant, timely, specific, and focused on the needs of the consumer (home huyers and sellers), huilders, and realtors.

\section{Summary of analyses already completed:}

1. Review of existing HERS/EEMs awareness campaign literature.

2. Revisw of HEAT SMART campaign.

3. Review of questionnaire response from Collaborative memhership. 


\section{Research still needed (if any):}

1. Review of finalized recommendations from HERS, EEMs and Implementation TACs.

2. Feasibility of establishing a national clearinghouse for HERS/EEMs information, as recommended in Issue Paper No. 52.

3. Feasibility of Collaborative membership being the primary vehicle for consumer education, through the network of their local membership organizations.

4. Potential cost of developing and fielding this information independently and/or through Collaborative membership activities.

5. How messages can he tailored to support existing efforts that have already achieved high levels of energ.' ifficient housing and/or how to effectively field a national campaign that contains messages aimed at several different audiences at several different stages in the buying/selling/remodeling process and at different levels of efficiency.

\section{Bibliography:}

1. Harrigan, Merrilee, "Moving Consumers to Choose Energy Efficiency."

2. Good Cents, "Good Cents" Promotional Folder and Sept. 1990 Publication.

3. Vories, Rebecca, Home Energy, March'April, 1989: "What Makes Ratings Systems Tick."

4. Infinite Energy: "Comprehensive Implementation and Promotion Plan for the Austin Energy Star Program," September 1984.

5. Philadelphia Electric Company, "Getting a Mortgage Just Got Easier," Excellence in Energy Efficiency Promotional Folder.

6. Brookhaven National Laboratory: "Do Your Part Heat Smart." 
Issue Paper No.: 5

Name of TAC: $\quad$ Awareness

Contact person: $\quad$ Randy Martin, Iowa Department of Natural Resources ${ }^{8}$

Date of issue paper: November 8,1991

Title of issue: $\quad$ What provision will he made for technical assistance?

Recommndations (if any) (include majority/minority): The task force recommends that a national HERS/EEM clearinghouse he established to provide technical assistance and to serve as a source of information to consumers and participating organizations. We recommend that the Department of Energy take the leadership role in seeing that this clearinghouse is established in the most cost-effective way.

Summary of analyses already completed: The following is a list of information that a HERS/EEM clearinghouse would need to have access to:

- Lists of contacts on the federal, regional, state, and/or local level for all current operating rating systems and for those areas that don't have a rating system.

- Guidelines for all approved rating systems.

- Lists of lenders in each state that are familiar with processing EEMs.

- Lending guidelines and information requirements for all national EEM programs.

- Fact sheets on HERS and EEMs for contractors, real estate agents, lenders, and appraisers.

- Fact sheets on all approved rating systems, including technical information on each.

- Fact sheets on how to apply for an EEM for both new construction and retrofit situations.

- Fact sheet on how to get an EEM when an approved rating system is not available.

- Fact sheets for consumers on common ways to improve a home's rating, such as foundation insulation, wall insulation, house tightening, furnace replacement, etc.

- Technical support staff who can answer questions on a timely basis for homeowners wanting to know how to implement some of the recommendations they've received.

- Energy-efficient construction technique and product information for contractors.

Alternative solutions with advantages and disadvantages: Each stakeholder's group could provide technical assistance to their own members. It would be difficult to provide consistent information and a lot of duplication of effort would result. Because of the duplication of effort, costs would he increased accordingly.

One of the participating organizations could step forward and agree to house and operate such a clearinghouse. This doesn't appear likely without funding from some source.

A new collahorative organization could be formed with funds provided by the participating organizations. This may he ideal, but will the organizations be willing to put funds toward it?

Staff at one of the existing energy information clearinghouses already funded by the Department of Energy could be trained to handle HERS/EEM questions and provided with the necessary technical information. This would appear to he the least costly approach.

${ }^{8}$ Currently with the U.S. Department of Energy, Denver, Colorado. 
Research Still Needed (if any): A determination of who is going to handle the clearinghouse, the yearly operational costs, and where the funding will come from. 
Issue Paper No.: $\quad 6$

Name of TAC: $\quad$ Awareness

Contact person: $\quad$ David Swankin, Consumer Federation of America

Date of issue paper: November 5, 1991

Title of issue: $\quad$ What are the best "trigger points" for awareness in the new and resale home sales process? How can these be activated?

Recommendations (if any) (include majority/minority): While every interest group has some role to play in assuring citizen awareness of HERS/EEM programs in the new and resale home sales process, there are a few critical trigger points where explanatory information must be given to consumers if the programs are to he widely utilized. We believe the following are the critical trigger points. For each trigger point, we have identified the interest group (or groups) that has (have) the most critical responsibility:

- Time of listing a home: real estate agents

- Time of making an offer: real estate agents

- Time of applying for a mortgage: banks, savings and loans, all primary lenders.

Summary of analyses already completed: Each trigger point analyzed. Matrix prepared.

\section{Alternative solutions with advantages and disadvantages:}

The TAC considered a wide variety of trigger points, including:

When buying a home

- Time of decision

- Offer submitted

- Apply for mortgage

- Home inspection

- Preclosing
When selling a home

- Time of decision

- Listing

- Contract submitted

- Counter proposal

- Inspection report received

At each of these points, one or more of the interest groups represented in the Collaborative Consensus Committee (CCC) can and should play a role in making consumers aware of HERS/EEM programs and opportunities. (See Awareness Issue Papers Nos. 2 and 3.) However, the TAC believes it better for CCC to identify the three critical trigger points listed in the recommendation above and to urge the key interest group(s) listed beside those three to recognize their special responsibilities.

Research still needed (if any): N/A 
Issue Paper No.: $\quad 7$

Name of TAC: $\quad$ Awareness

Contact person: $\quad$ Bill Griffin, Commonwealth Edison

David Swankin, Consumer Federation of America

Date of issue paper: November 5, 1991

Title of issue: $\quad$ "Labeling" criteria

Recommendations (if any) (include majority/minority):

1. Criteria need to be set for both "promotional labeling" (i.e., labeling used in advertising, promotional brochures) and "technical" labeling (i.e., detailed informational dealing with the component elements of a rating).

2. Because some rating systems are designed on a pass-fail basis, while others are designed as scaled systems, it is essential that citizens be able to compare different systems. All promotional labeling needs to include in a prominent place:

a. A common universal comparability number that translates pass-fail systems to a scale;

b. A standardized display of dollar savings or costs per year.

3. All programs, whether existing now or developed in the future, that comply with the Collaborative Consensus Committee adopted program criteria should be allowed to display on their promotional and technical labels a "logo" or "seal of approval," similar to the "UL" safety mark on appliances.

Summary of analyses already completed: Group has examined many existing labeling programs and labels, and is struck by lack of standardization at the present time.

Alternative solutions with advantages and disadvantages: Given that a variety of programs will be able to co-exist, it is imperative that the "standardized" criteria recommended above be made part of the program. Failure to do so will result in citizen confusion.

Research still needed (if any): Model labels with alternative ways of addressing the recommended criteria need to be field tested so that the final "approval" format will be the one that is most understandable to the public. 
Issue Paper No.: $\quad x$

Name of TAC: $\quad$ Awareness

Contact person: Bill Prindle, Alliance to Save Energy"

Date of issue paper: July 16,1991

Title of issue: $\quad$ What labeling should he required for a home energy rating?

Summary of analyses already completed: In any consumer-oriented rating program, the label is the most important piece of information. It is usually the only information the consumer sees, regardless of the quality or extent of the work done by the rating organization and its elaborate skills and tools. So getting the right kind and amount of information on the label, in a form that is uscful to consumers and others involved in the home sales process, is ahsolutely essential.

The label should also be consistent and easily identifiable, so that consumers and shelter industry professionals come to know and look for it. Since some rating systems are already in use with their own lahel formats, some accommodation must he made that will not disrupt ongoing programs while fulfilling the need for a consistent home energy laheling format for the country.

There are three kinds of information that should he included on the lahel to keep it simple while making it useful to consumers and shelter industry professionals:

- Rating scale designation-Assuming the HERS guidelines set a common scale for reporting energy efficiency, the lahel must prominently display this information so that it is physically readahle and comprehensible.

- Estimated energy costs - Assuming HERS guidelines require calculation of estimated energy costs, this information should also be displayed.

- Component efficiency information--A single number or other hit of information pegging the home to an efficiency scale is useful for comparing the home to other homes at a point in time. However, it is also important to know what is behind the rating: the insulating value of walls, ceilings, floors, doors and windows, the type and efficiency of heating and cooling equipment, water heater efficiency, information on special features such as solar equipment, etc., can he important for giving the rating lasting value. Appraisers, utility people, and others can use the hasic component information over time, to re-rate the house, for future surveys of home energy characteristics, to make additional calculations for future retrofits, and other purposes. The value of this information should he halanced against its potential complexity. The label should not he overloaded with data in such a way as to make it unreadable. The Bonneville Super Good Cents label (copy attached) is an example of a lahel that includes component information; since this is a certification-type HERS, it doesn't include a scaled rating or cost projection.

\footnotetext{
"Now with Barakat and Chamberlin, Washington, DC.
} 


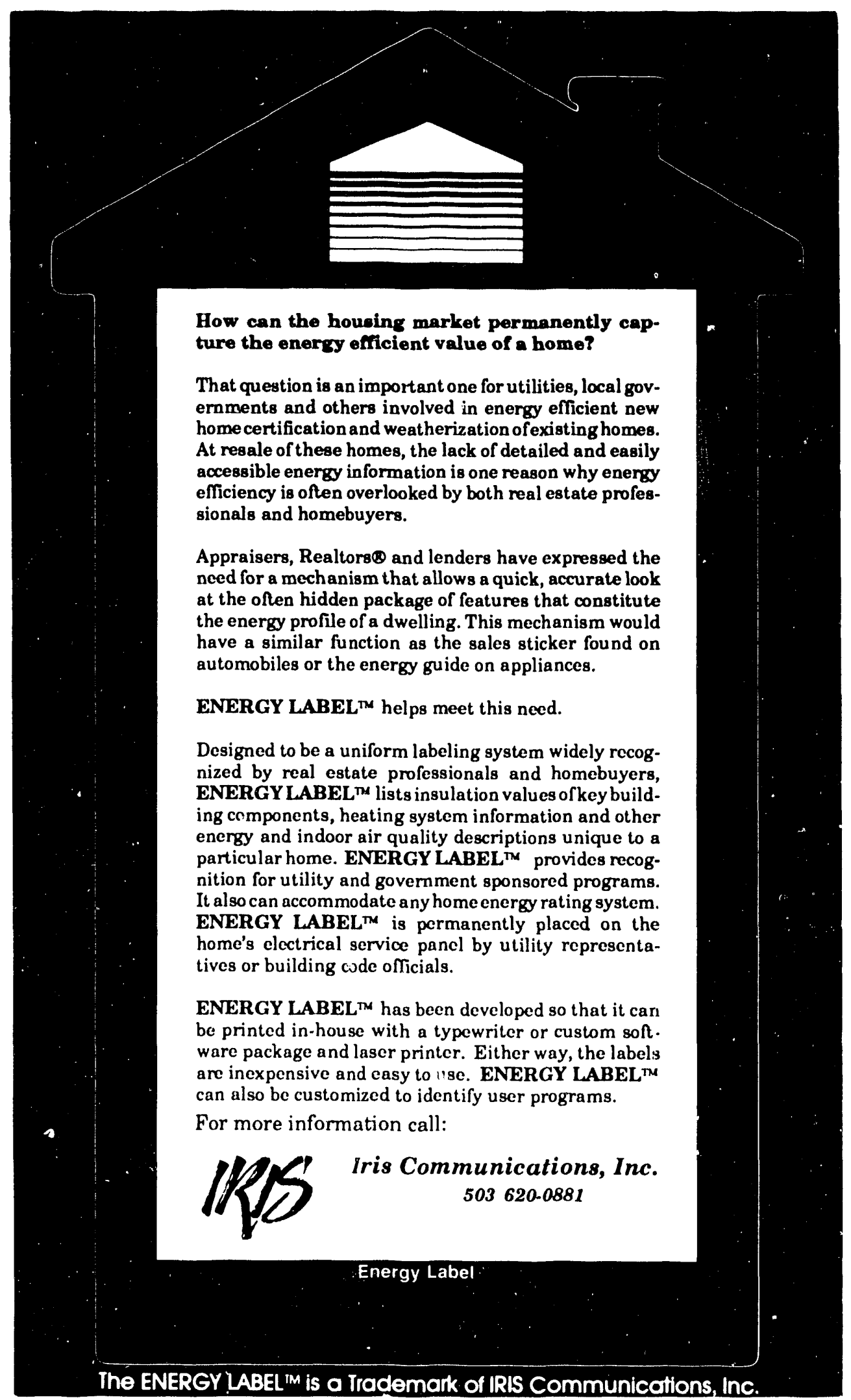


Issue Paper No.: $\quad 9$

Name of TAC: $\quad$ Awareness

Contact person: $\quad$ Mike Lennon, American Society of Home Inspectors

David Swankin, Consumer Federation of America

Date of issue paper: November 5, 1991

Title of issue: $\quad$ What proficiency requirements should there be for raters and anyone making calculations on retrofits?

\section{Recommendations (if any) (include majority/minority):}

1. For new homes, training needs of auditors are likely to be far less demanding than for auditing existing homes. The Collahorative Consensus Committee should set as a criteria that all new homes' rating systems include a written set of instructions, including sufficient background materials, to assure accurate audits.

2. For existing homes, we are extremely concerned that huilding denigration (via rot and moisture problems) and occupant safety and health (via fire, combustion products, and other air quality issues) are highly likely to be compromised via "energy saving" retrofits. It is our recommendation that appropriate training/certification materials be develoned and implemented for all energy raters. Our research indicates that with proper materials, this would add one full day of training time per energy rater. The data needed for such training material development is readily available; little additional research would be required.

\section{Summary of analyses already completed:}

1. Determined that the training for most current programs, no matter how good they may he, are not documented and therefore are unavailable for use.

2. Analyzed auditor requirements of major existing programs.

3. Developed an "ideal model" that would combine ability to perform energy audits with ability to perform related health and safety audits.

\section{Alternative solutions with advantages and disadvantages:}

1. Advantage of "high standard" is that it will assure consumer confidence, avoid "bad name" many think plagued the Residential Conservation Service program in the 197()s due to incompetent audits.

2. The hetter the audit, the more the financial industry is likely to make linancing available.

3. Disadvantages of setting standard too high are:

a. Costs of the audit could he quite high.

h. Availability of sufficient pool of qualified auditors may he limited.

\section{Research still needed (if any):}

1. More information on cost and availahility impacts. 


\section{Section 3}

Special Papers 
Special Paper No.: 1

Contact person: $\quad$ Perry Bigelow, National Association of Home Builders (Bigelow Homes, Inc.)

Date of issue paper: December 2, 1991

Title of issue: $\quad$ Characteristics of an energy-efficient mortgage program

The energy-efficient mortgage program shall have the following characteristics:

1. In the loan qualification process, the energy savings shall be recognized as follows:

A. An energy-efficient home is a home that has heen built or improved to at least a nationally recognized voluntary consensus energy performance standard. For an energy-efficient home, the energy savings shall be recognized as follows:

1. By allowing a $2 \%$ increase in the basic qualifying ratio, and

2. By allowing any additional energy savings beyond those achieved by building or improving the residence to a recognized energy performance standard to be deducted from the normal housing costs (PITI) included in the qualifying ratio before calculating the ratio.

B. For existing homes that do not meet a nationally recognized voluntary consensus energy performance standard, the energy savings may be recognized at purchaser's election by allowing the energy savings from making energy-efficient improvements to be deducted from the normal housing costs.

C. Underwriting procedures shall recognize energy savings separately and independent of compensating factors; cnergy savings are to be deducted from the expense side of the basic qualifying ratio.

2. For the purpose of adding the cost of cost-effective energy savings construction or improvement features to the mortgage and determining the maximum loan available on a home with energy saving construction or improvement expenditures, an principle, an amount up to the present worth of the capitalization of the energy savings may be added to the mortgage, the maximun amount of which has been theretofore determined on the basis of the loan-to-value ratio of the appraised value.

A. On an energy-inefficient home, an amount may be added to the mortgage that is equal to the sum of the cost of energy saving improvements up to a maximum amount at which the savings will pay for the cost on a monthly basis when amortized in a 30-year mortgage at the mortgage's initial interest rate.

1. Said mortgage increase shall not exceed $\$ 5,000 \mathrm{cr} 5 \%$ of the appraised market value (whichever is greater), not to exceed $\$ 8,000$.

2. This amount may he added to the loan after the appraised value has heen determined and after the applicant's hase loan has been approved.

3. The cost of energy improvements shall he escrowed at the lender title company or other suitable depository at closing.

B. On an energy-efficient home, an amount may he added to the mortgage that is equal to $5 \%$ of the appraised value.

C. The risk of loss due to default of the mortgage on that part of the amount so added to the mertgage that exceeds a threshold loan-to-value ratio shall he covered by supplementary mortgage insurance for a period of five years. 
D. The borrower's down payment on a 95\% loan for the incremental increase in the loan and mortgage as described above shall be provided as follows:

1. If acceptable to the lending agencies and mortgage backed securities industry, it shall be added to the loan and covered by a special mortgage insurance pool.

2. If acceptable to the lending agencies and mortgage backed securities industry, the incremental down payment shall be provided by a grant from a federal, state, or other public or private program created for said purpose. Private programs may include utility companies, home builder associations, and others who have an interest in promoting energy savings.

3. A mortgage on an energy-efficient home shall be able to exceed the maximum mortgage limit in effect at the time by up to $5 \%$.

4. The energy-efficient mortgage program shall have the following general characteristics:
A. It must be available from all major lending and insuring agencies and all regular types of mortgages.
B. It must be as uniform as practical among different types of mortgages and different lending and insuring agencies. It must provide consistent relief and benefit to borrowers who purchase energy-efficient homes and to borrowers who want to make energy saving improvements to nonenergy-efficient homes.
C. It must be administratively simple to apply. The forms, policies, procedures, rating system, etc., should be uniform and as streamlined as possible.
D. Every Tax-Exempt Mortgage Revenue Bond program shall have an energy-efficient mortgage program as described herein.
E. Alternative approaches may be used provided that they are at least equivalent in characteristic to the provisions contained herein.


Special Paper No.: 2

Contact person: $\quad$ Perry Bigelow, National Association of Home Builders (Bigelow Homes, Inc.)

Date of issue paper: October 30,1991

Title of issue: $\quad$ Overcoming the barriers and disincentives to the purchase of energy-efficient homes with energy-efficient mortgages

Before we can design an effective energy-efficient mortgage, we need to know what one is. At the most basic level, an energy-efficient mortgage mans a mortgage on a residential property that recognizes the energy savings of a home that has energy saving construction or improvement features and that has the effect of continuing to qualify borrowers who, but for the energy saving construction or improvement expenditures, would otherwise have qualified for a mortgage. Therefore, an energy-efficient mortgage is one that removes the mortgage barriers and disincentives to the purchase of energy-efficient homes, and that removes the mortgage barriers and disincentives to making energy-efficient improvements in existing homes.

The most basic disincentive that energy-efficient homes face is that home purchasers who would otherwise be interested in an energy-efficient home do an end run around the mortgage system in order to buy a larger or better appointed home (more bedrooms, baths, etc.) instead of an energy-efficient home, since they want to buy as much house as they can qualify for. Put another way: most buyers spend as much of their income on housing as they think a lender will lend them. If you think about the decision-making process you went through when you bought your first home, you'll understand what happens. It costs more to install the construction features that make homes energy efficient, and since you had a fixed number of dollars to spend, you would have had to accept a smaller or less well appointed home with fewer bedrooms or bathrooms, etc., in order to qualify for the loan. Which home would you have bought? Every person to whom I have ever asked this question has said he would have bought the larger or better appointed home since he would only live in it a few years (seven is the average); since he needs all the space and features he could afford, it is against his best interest to make this sacrifice.

The end run occurs because he can increase the percentage of his income spent on total housing by buying an energy gizzling home and paying higher energy bills; since his goal is to spend more of his income on housing than the system allows, the nonenergy-efficient home becomes the tool he uses to accomplish this objective. The result is that energy-efficient homes are less competitive and less affordable. This problem goes to the heart of each of the disincentives and barriers, and it illustrates the inadvertent, inherent bias that exists in the mortgage-making system, which assumes that all homes use a lot of energy and were therefore lumped together-all homes did use a lot of energy when the present ratios and guidelines were established.

In the mortgage process, there are five barriers and disincentives to the financing of energy-efficient homes. Nonenergy-efficient homes that are being improved have these barriers plus others that relate to the nature of marketing the necessity of energy improvement to sellers and/or purchasers and getting the improvements approved in the mortgage process and installed on a house-by-house basis, all in a timely way. The first barrier is the cause of the other four, which keep purchasers from purchasing energyefficient homes with energy-efficient mortgages. The five barriers and disincentives are as follows:

1. Lender's risk of default or risk of loss due to default: The lender and/or mortgage insurer cannot be expected to increase his risk without additional compensation. 
2. Qualifying ratios (stretch, PITI-ES, PITI+E, or some combination): This is the necessity to deduct the energy savings from other expected, normal housing costs included in the qualifying ratio (PITI) in order to offset the additional construction costs.

3. Financing the improvements: This is the necessity to include the added cost of energy-efficient improvements in the mortgage.

4. Down payment: This is the necessity to not increase the purchaser's down payment due to the increased sale price due to the cost of energy-efficient features; the greatest impediment for a first time buyer to purchasing a home is coming up with the down payment.

5. Increase maximum mortgage limits: This is the necessity to allow mortgages to exceed their present limits for energy-efficient homes.

Our goal must be to create a comprehensive, synergistic energy-efficient mortgage program that overcomes all of these barriers in such a way that all of the costs of the program (whether they he energy improvements or lender risk) are paid for by the energy savings. 
Special Paper No.: 3

Contact person: Jim Curtis, Association of Energy Efficient Mortgage Service Companics

Date of issue paper: October 10, 1991

Title of issue: $\quad$ Barriers to energy-efficient mortgages

Barriers to Energy-Efficient Mortgages (EEMs) - when EEMs mean allowing home buyers and homeowners to add the cost of cost-effective energy-efficient improvements ${ }^{10}$ to their first mortgages when their homes are not energy efficient. This paper assumes consumer awareness that lenders and realtors have already achieved.

1. VA (accounting for $5 \%$ of $6 \mathrm{mn} / \mathrm{yr}$ loans) allows financing of $100 \%$ of the cost of energy improvements. (Only 2,000/yr are processed as EEMs.) FHA, Fannic Mac, and Freddie Mac require from $2.5 \%$ to $70 \%$ down payment on the cost of improvements. The required down payment is a barrier to many customers.

2. In the VA loan program, the customer has 4 weeks to decide without delaying the loan; the FHA program permits $2-1 / 2$ to 3 weeks to decide; and FNMA/Freddic Mac permits 2 days. More information is needed for an EEM than for a regular loan, such as a home energy rating, specification sheets for improvements being installed, letter of instructions from the consumer to the lender on the improvements intended and their cost, appraisal reports for the loan types that require it, and certification of completion of work reports for Fannie Mae and Freddie Mac. In aúuition, lender instructions to the title company must be transmitted in some types of loans.

3. The effective maximum VA loan amount is $\$ 184,000$. FHA has regional maximum loan limits, which are $\$ 124,875$ in the lower 48 states. FNMA and 5 reddie Mac have a maximum loan limit of $\$ 191,250$. On a national basis, $6 \%$ to $10 \%$ of all loans are at the maximum allowable loan limit (across all programs). In some metropolitan areas at given points in time, a large proportion (say, $50 \%$ ) of loans are at the maximum loan limit. This effectively excludes them from financing additional costs for energy improvements.

\footnotetext{
11)"Cost-effective" energy improvements are those that will produce an immediate positive cash flow monthly savings on utility hills are greater than the increase in monthly mortgage payment due to the increment in mortgage payment as a result of financing $100 \%$ of the cost of the improvement.
} 
Special Paper No.: 4

Contact persons: $\quad$ Tom Farkas, Edison Electric Institute

Doug Swartz, City of Fort Collins Light and Power

Ken Crandall, U.S. Department of Housing and Urhan Development

Jim Fulkerson, CHERS, Inc.

Date of issue paper: December 13, 1991

Title of issue: $\quad$ Is a single "rating method" necessary for uniformity?

Issue: A "rating method," as used here, is the equation and associated inputs that describe the relationship of a rating to an amount of energy use, energy cost, or other measure of efficiency. There are many different rating methods that could be chosen.

Recommendations (if any) (include majority/minority): Yes, with a clarification that local systems must be able to use any designation they choose to present rating information to users within their service area. A single rating method would facilitate linkage to a uniform national HERS/EEM program. Further study is recommended to determine the form, parameters, and inputs of the rating method.

Discussion: Several arguments support a uniform rating method:

1. Secondary lenders need uniformity for EEM lending practices. A uniform approach, if carefully thought out, should be able to mean that a given rating in one region conveys the same information from a lending standpoint that it does elsewhere. This consistency will simplify training of agency field office personnel, builders operating in more than one area, and other housing industry participants.

2. A uniform approach could rationalize the market for systems coming on line in the future. Decisions regarding the rating method are never easy for a developer, because there are so many options and trade-offs-lots of grey areas. A uniform national approach could provide guidance and eliminate some of those quandaries. It also avoids the potential of an even greater plethora of rating definitions than is now being used.

3. A national data base will be much more straightforward to operate with a uniform approach.

4. A uniform approach could make a national marketing program for HERS/EEMs more feasible.

Providing linkage between HERS and EEMs and being responsive to the needs of the secondary lending agencies is the most convincing argument. The other three arguments together look to the future. Use of a uniform approach should facilitate a more rapid adoption of HERS/EEMs on a nationwide basis.

There are some caveats regarding existing systems. Though most existing HERS have independently settled on a 100-point scale, the equation and inputs behind the scale are not the same among independent systems. Further, many have also chosen to present other rating designations (e.g., stars) as the primary output to users. There have been good reasons for these decisions. In terms of enabling systems to conform to a national approach yet accomplish local marketing goals, it is important to have a uniform rating method for use with lenders, a data base, etc. - while allowing HERS operators to choose whatever rating designations they wish to communicate to local end users. 
Some existing programs may choose to give up their existing rating method in favor of the uniform national one. This may result in altering existing ratings. If so, it may require re-educating users 10 a different scale, re-issuing ratings that have already heen performed, etc. Each system will have (o) weigh these cons against the potential benefits of affiliating closely with the uniform HERS/EEM program. 
Special Paper No.: 5

Contact person: $\quad$ Tom Farkas, Edison Electric Institute

Date of issue paper: November 8,1991

Title of issue: What is a rating model or rating method? Is it necessary for the Collaborative Consensus Committee (CCC) to select one to establish uniformity?

Recommendations (if any) (include majority/minority): Yes. It is necessary for the CCC to recommend a rating model (rating method) in order to provide for the necessary uniformity appropriate for linkage to the mortgage process :vhile still allowing for local flexibility.

Summary of analyses already completed: A rating model describes the relationship hetween the house being rated, the candidate house, and itself at a benchmark or reference condition. The rating model can be defined by an arithmetic equation. It can be shown as a sloped line, which is a graphic representation of the equation. The sloped line is a depiction of a graduated rating scale, showing the relationship of a rating to an amount of energy consumption, energy cost, or a measure of efficiency, such as percentage.

Attached is a representative sample of a number of different rating models. Some of them represent models that are already used in different rating systems that exist around the country; others are offered to the CCC for comparison purposes.

Distinguishing properties of the rating models are: whether it has an open or closed scale; whether it has its reference points defined by selecting a henchmark near the middle of the line or at its end points; whether the rating has as its measure units of energy consumption, energy cost, or as a ratio of efficiency or percentage of a house's performance compared to its reference condition.

As can be seen in the attached figures, there is no uniformity among the rating scales, even those scales that use a $0-100$ closed method. It is the rating model that needs to be uniform, not just its numbering system.

Aspects of a rating model (rating method) to be recommended for selection by the CCC:

- Estahlish uniformity across, as well as within, the rating scale

- Be hased on nationally recognized consensus standards

- Provide for the treatment of fuel neutrality

- Encourage the use of higher energy efficiency construction techniques and the use of higher energy efficiency products and equipment

- Be self-adjusting to automatically recognize advances in energy efficiency

- Be hased on a common threshold for energy efficiency

- Not artificially compress efficiency potential of huilding design, components, or equipment nor preclude incorporation of advanced technology henefits

- Provide for a simple, casy to understand reporting index. 


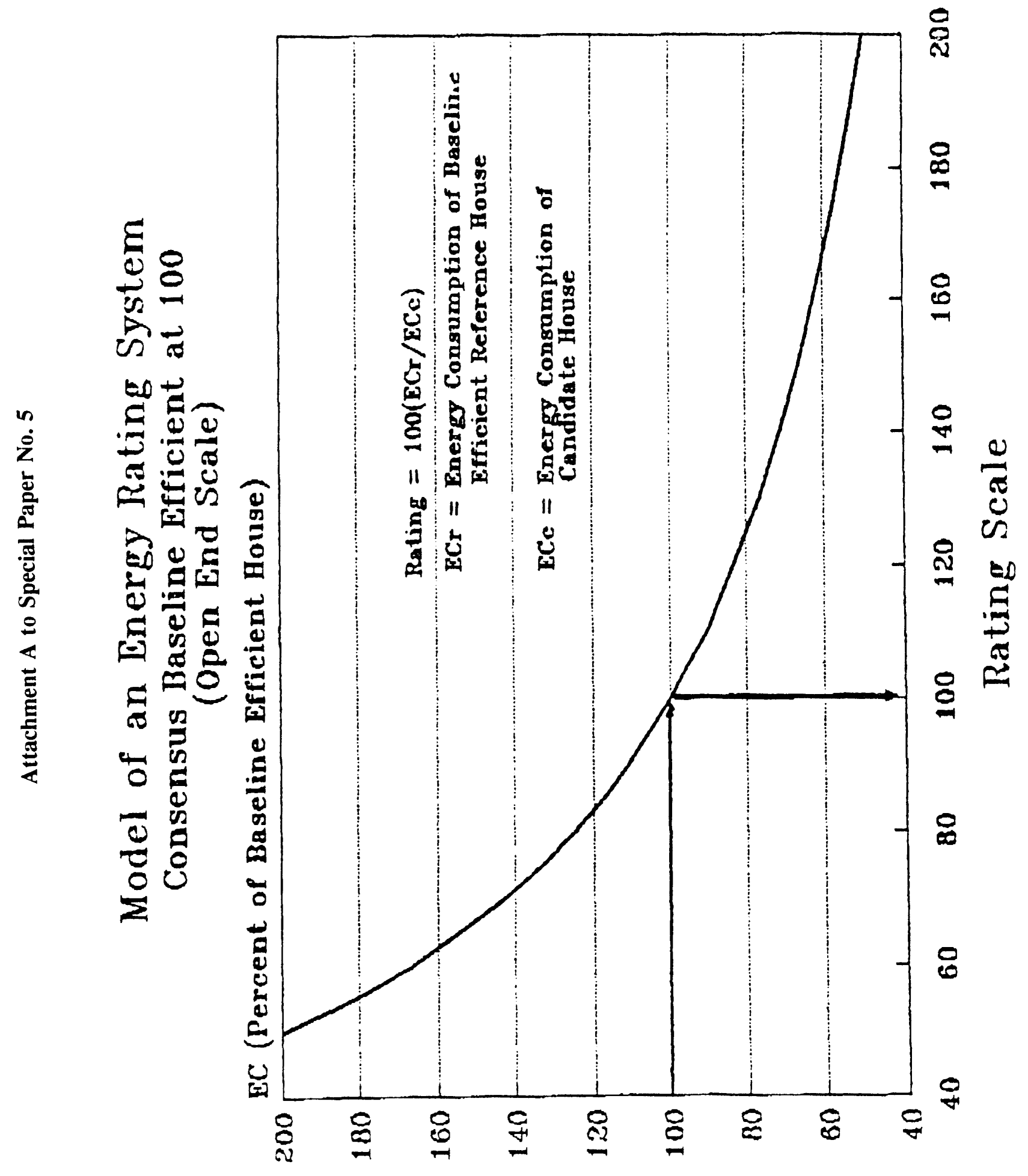




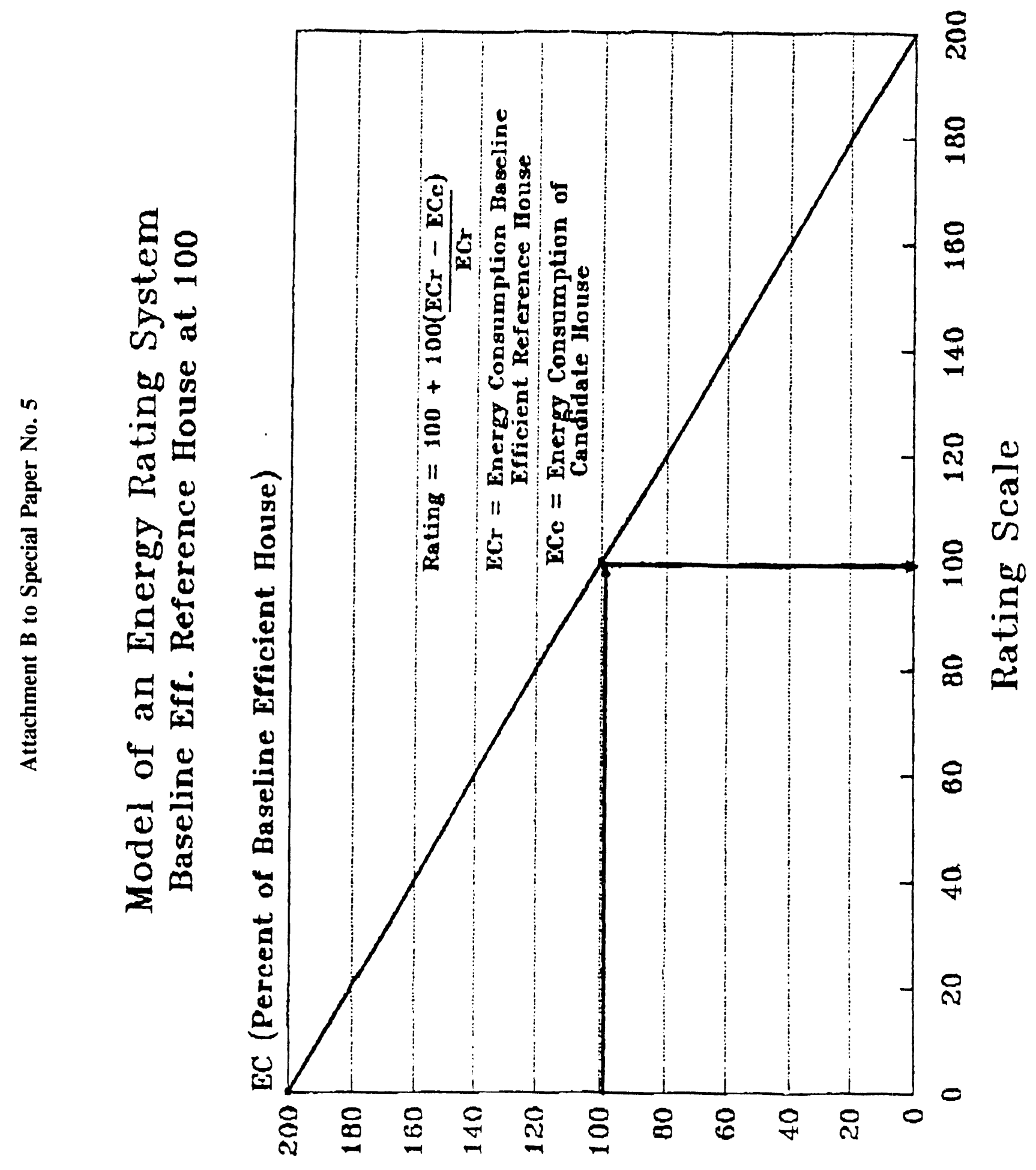




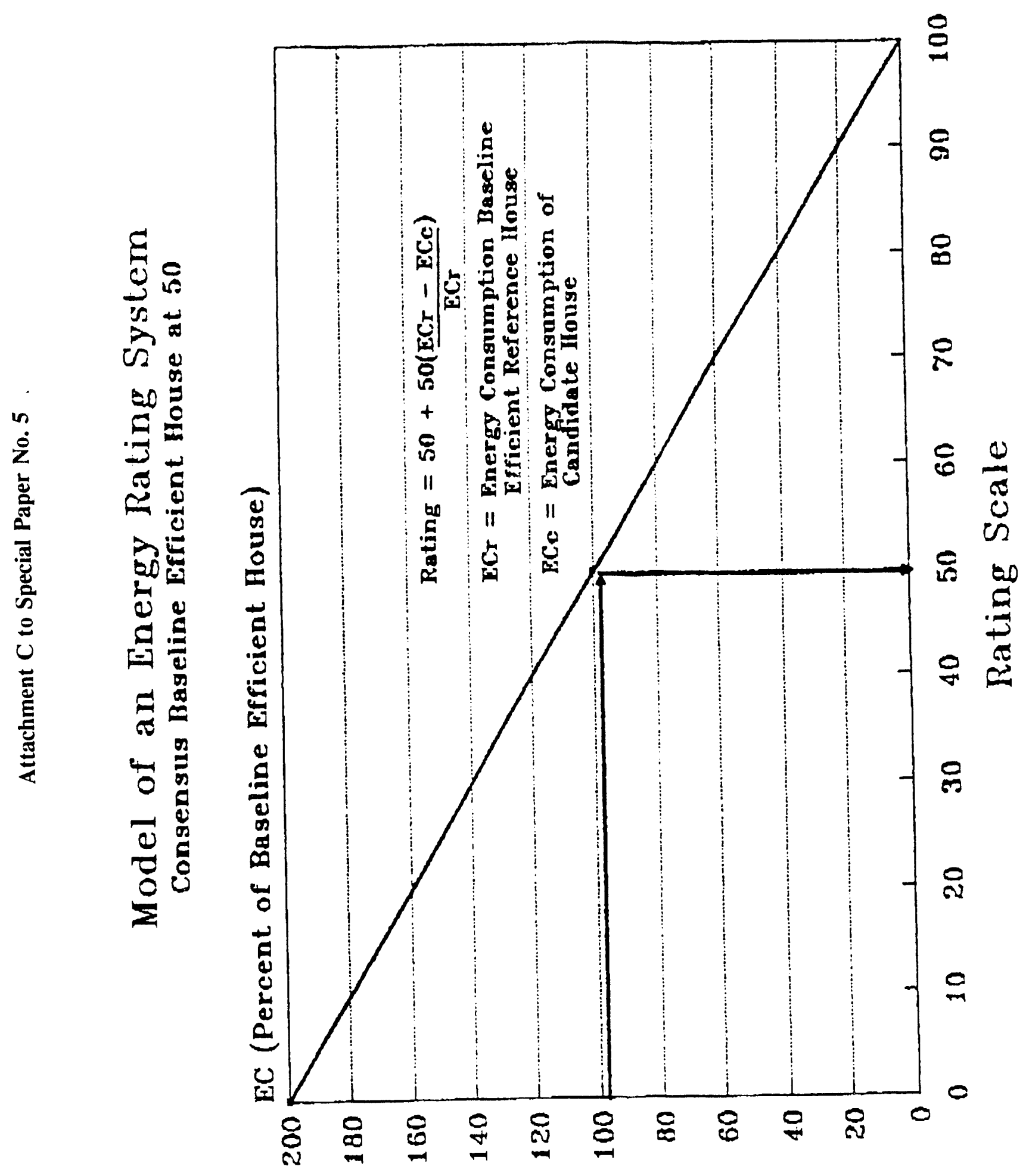




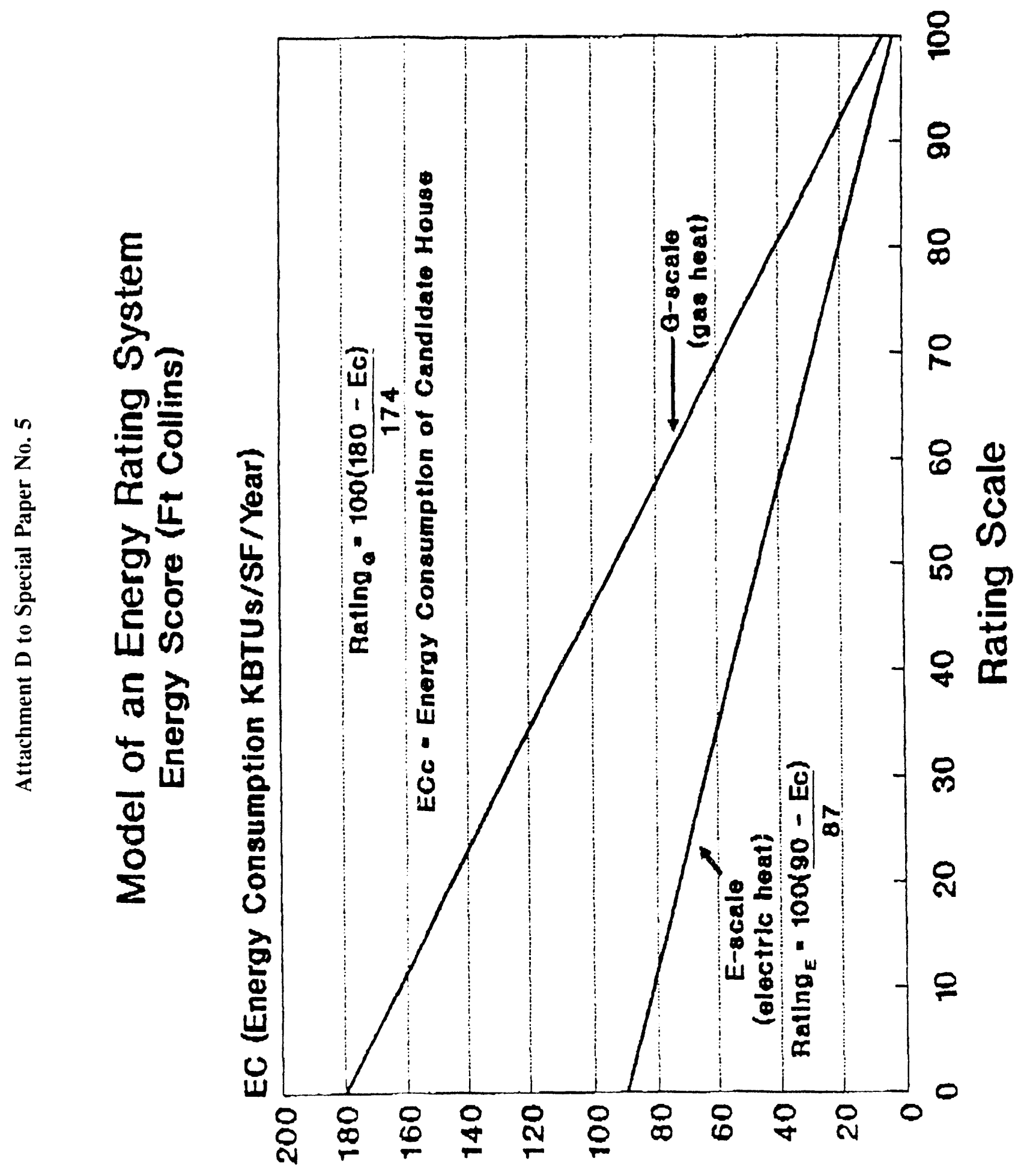




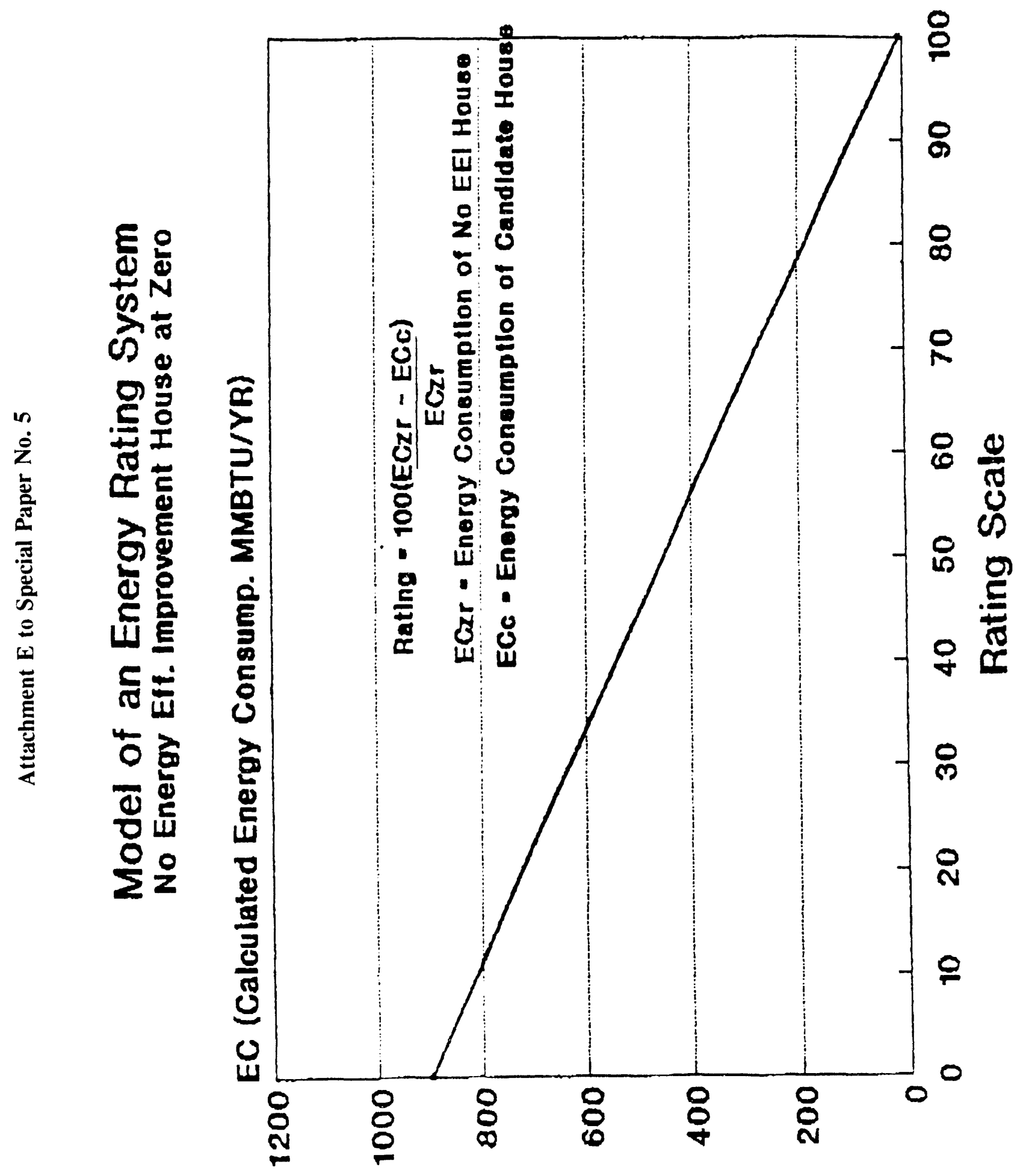




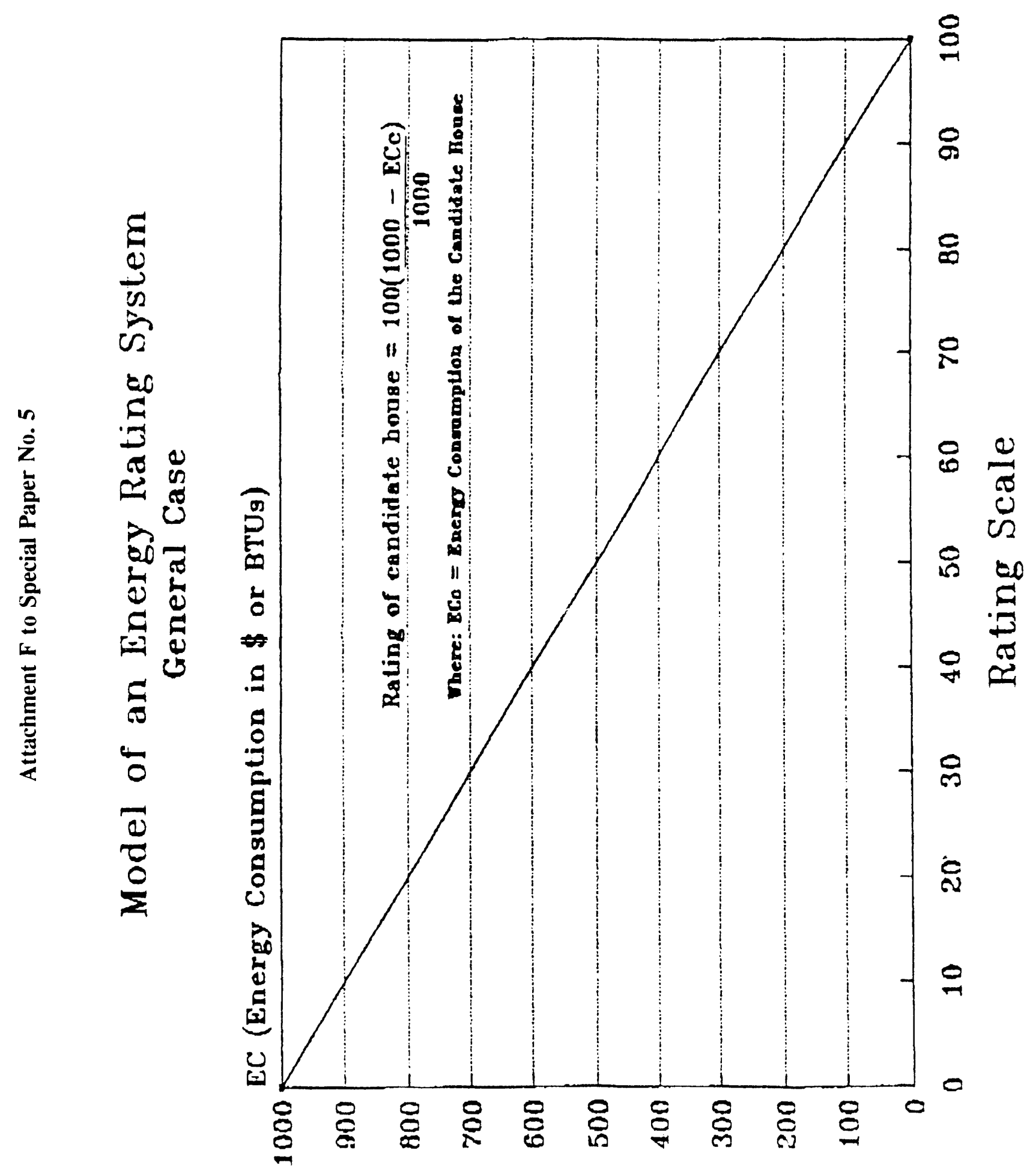


Special Paper No.: 6

Contact person: Benny Folsom, Southern Electric International,Good Cents Division Ron Hughes, Energy Rated Homes of America

Date of issue paper: October 29, 1991

Title of issue: $\quad$ Rater training/qualifications

Recommendations (if any) (include majority/minority): Rater training/qualification should include a mix of classroom and on-site training, concluding with certification by testing. An ongoing quality assurance program will ensure long-term acceptance of rating integrity by lending agencies.

Summary of analyses already completed: Average rater qualifications exist among the persons now

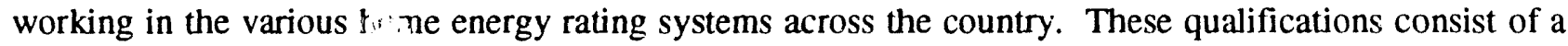
basic understanding of the following:

- Residential construction techniques - current and historical

- Heat transfer theory, moisture, and vapor control

- Heating, ventilating, and air conditioning system sizing, air disuribution system efficiency, testing

- Energy efficiency measures

- Residential real estate appraisal methodology

- Energy-efficient mortgage requirements.

Training required to achieve this understanding should include:

- Load calculations course (i.e., Manual "J")

- Duct design course (i.e., Manual "D")

- Residential building technology course

- Energy auditing/reporting

- Operation of rating software/tool.

Other Information Still Needed: Consensus recommendation on what rating methodology will be utilized (i.e., How does a rater transform a house into a "score"?) in order to better determine rater training/qualification needs. 
Special Paper No:: 7

\section{Contact person: Charles Fritts, Federal Home Loan Mortgage Corporation}

Date of issue paper: November 12, 1991

Title of Issue: $\quad$ Special paper from the lenders' caucus

On November 7, 1991, representatives of Freddie Mac, Fannie Mae, FHA, VA, and FmHA met. The purpose of the meeting was to discuss the efforts of the Collaborative and the positions of these groups relative to the Collaborative. The organizations also sought to identify any grounds for developing a consensus on issues before the Collaborative.

The organizations that met agreed that energy efficiency in housing should be promoted and are willing to make efforts to achieve that goal. They are cognizant of the interests of the members of the Collaborative who strongly support energy efficiency in housing. They are also concerned that a great deal of information has not been collected about energy use in housing, occupant behavior, the use of energy-efficient mortgages (EEMs), and the related risks.

The organizations that met consider themselves the ultimate risk holders in the mortgage lending process because they guarantee or insure mortgages, as well as absorb losses due to mortgages' defaults and foreclosures.

The risk holders perceive that efforts to promote energy-efficient mortgages could result in an increase of their risk posture. EEMs, depending on the modification of the underwriting standards, generally require a relaxation of those underwriting standards. Perceptions aside, it is certain that very little data exist to be able to evaluate the risk characteristics of EEMs. As ultimate risk holders, these entities are unwilling to assume unknown risks by modifying underwriting standards without the necessary data to evaluate those risks.

A goal of the meeting was to be able to present the Collaborative with steps the risk holders can take at this time to promote energy efficiency in housing, within their current risk management standards. Another goal was to provide a summary of significant issues that the risk holders would require to enable them to support the efforts of the Collaborative.

Based on the foregoing statement of the risk holders' financial position and on the information that is currently available, a consensus was reached on several general issues pending before the Collaborative.

Steps that the risk holders can take to promote energy efficiency.

1. Common standards among Fannie Mae, Freddie Mac, FHA, VA, FmHA The risk holders can work to develop common standards, forms, and practices to make their respective EEM programs more user friendly.

2. Removal of barriers

Each risk holder can review its existing program in an effort to remove any unnecessary harriers and simplify the process.

3. Sponsorship of educational, training, and promotional programs

Each risk holder will make efforts to promote the use of EEMs hy increasing training and educational programs about them. 


\section{Data collection}

The risk holder could take steps to collect information on their EEM programs and provide data to a single party for further analysis.

\section{Analysis of existing HERS/EEMs data to evaluate loss experience}

The risk holder can take data that may exist concerning homes that have had an energy rating and/or obtained an EEM, and cross-check those loans against the records of the loans they currently hold to generate current performance information.

Based on the current standards of risk management, the following are the significant issues where consensus was reached among the risk holders in order for them to support the efforts of the Collaborative. (The risk holders also note that each retains the right to modify its program for such purposes as experimentation, risk management, business development, etc.)

1. Appraisal issue: market value or cost of improvements

The value of the property must be based on its market value. Any increase in the value due to energy-efficient improvements must be based on the market response to the improvements, rather than the cost of the improvements themselves.

2. A HERS must precede an EEMs

With regard to existing homes, a HERS must be in place before a broad based EEM system can be established.

3. Essential elements of a HERS

A HERS must have credible standards and an effective quality control mechanism.

4. Actual savings, increased ratios, fixed ratios

Actual savings should be used as a separate factor in calculating whether a borrower qualifies. The use of energy savings will be in the underwriter's judgment. The group cannot accept an automatic increased ratio or a fixed formula to accommodate energy savings, although some risk holders have chosen to do so in certain cases.

5. Treatment of energy savings versus other factors

Energy savings is a factor in the underwriting process. It is not a fixed, mandatory expense like PITI.

6. Different standards for new versus existing houses

The group recognizes that new houses may be built to a more energy-efficient standard than most existing homes. Risk holders may employ different practices to evaluate the efficiency of the two, with the intent of achieving verifiable energy efficiency.

7. Definition of the base house

- With regard to existing homes, minimum energy efficiency standards should be established for use as a base to determine whether a house is energy-efficient and to what degree.

- With regard to new homes, the use of a recognized, energy-efficient, construction standard would he acceptable, preferably used in conjunction with a HERS.

8. Caps on cost of improvements

There should not he a limit on the cost of improvements as long as market value appraisals and proper underwriting are used. 
Special Paper No.: 8

Contact person: $\quad$ Fulkerson Task Force (Jim Fulkerson, California Home Energy Rating Systern, Inc.)

Date of issue paper: December 17, 1991

Title of issue: $\quad$ Consensus statement on a national consensus standard for HERS/EEMs

"There needs to be a nationally recognized consensus standard as a reference for the purpose of determining efficient versus nonefficient and as a factor in calculating energy savings that can be recognized in the energy-efficient mortgage."

Other Pertinent Points:

1. Additional work will be required to arrive at a reference and to assess its impact with respect to mortgages.

2. A distinction between "reference" and "baseline," for the purposes of clarity, is appropriate. Reference is the nationally recognized consensus standard that determines efficient versus nonefficient. Baseline is the point from which energy savings are measured. For nonefficient homes, where the intent is to make cost-effective energy-efficient improvements, the baseline is the house as it actually exists. For efficient homes, the baseline will be an as yet undefined point that has an agreed upon economic relationship to the reference. 
Special Paper No.: 9

Contact person: $\quad$ Ron Hughes, Energy Rated Homes of America

Date of issue paper: October 23, 1991

Title of issue: $\quad$ What could an infrastructure for national implementation and administration of a HERS/EEM program look like?

Recommendations (if any) (include majority/minority):

- A HERS/EEM National Oversight Committee would be established to provide guidance and oversight.

- The National Renewable Energy Laboratory would work with the HERS Technical Advisory Committee (TAC) in developing procedures for technical approval of rating tools. The technical approval procedures would be piloted by evaluating HERS analysis tools currently in use.

- A national HERS program would be coordinated, implemented, and administered through a nonprofit HERS Council comprised of member HERS programs in each state and members of national HERS programs (e.g., Good Cents, Energy Rated Homes of America [ERHA], National Association of Home Builders [NAHB]/Thermal Performance Guidelines, etc.), with directors drawn from this memhershin.

- Within each state, a local nonprofit membership organization would be established, with directors drawn from the shelter industry to coordinate, approve, and quality control HERS programs within the state. Until such an organization was established in each state, local EEM-linked HERS programs would relate directly to the national HERS Council or through a national HERS program.

- State energy offices would coordinate with the nonprofit HERS organization in each state to approve one scaled rating system (in states desiring a scaled program) and any number of certification programs within the state for use with EEMs.

- TACs would continue in order to be of assistance to the HERS/EEM National Oversight Committee and the HERS Council.

- Initial funding would come from the Department of Energy (DOE), states, the mortgage industry, private foundations, and elsewhere for initial operating expenses and to establish infrastructure.

- The HERS Council would work with existing HERS programs to provide regional coordination, training, and technical assistance to states and jurisdictions starting HERS/EEM programs.

- New EEM procedures based on "savings" would he piloted in states where rating systems are in place or being piloted.

- The HERS Council would maintain a national data bank of the energy characteristics and energy costs of rated houses and include all houses financed with an EEM.

Question: Should the HERS Council be expanded to a HERS/EEM Council with EEM members as well as HERS inemhers? Or should there he a totally separate EEM umbrella organization? 


\section{Functions:}

Functions of the different groups could include the following:

\section{HERS/EEM National Oversight Committee (NOC)}

The Collaborative Consensus Committee (CCC) could evolve into the NOC. It would include all of the stakeholders who would be willing to participate. This group would convene two to four times the first year and once or twice a year after that. These individuals have other jobs and responsibilities within their own fields. Most of the work of this group would be to advise and monitor.

Objective: Serve as a steering committee to assist and encourage national implementation and use of HERS and EEM programs by:

- Providing consulting as needed;

- Providing guidance on HERS/EEM goals, objectives, and strategies;

- Encouraging use of HERS/EEM programs within NOC member industries;

- Assuring stakeholders' needs are met and concerns addressed.

\section{Education/Awareness/Training TAC}

This would be composed of consumer representatives and representatives of industries that would benefit from training to make the HERS/EEM concept successful within their industry.

Ohjective: Promote awareness, education, and training for consumers and within the shelter industries through:

- Advising on designing educational materials to meet the needs of lenders, appraisers, builders, real estate agents, utilities, consumers, etc.; and

- Encouraging and coordinating training within industries.

\section{EEM TAC}

This would he composed primarily of representatives from the mortgage lending, mortgage insuring and guaranteeing, and secondary mortgage market.

Objective: Encourage more affordable housing and improved loan security through use of EEMs and HERS hy:

- Modifying mortgage underwriting procedures to hetter consider the energy savings that result from improved energy efficiency;

- Assisting in coordinating pilots for incorporating HERS/EEM programs within industry programs (i.e., affordable housing initiatives, first-lime home huyer programs, property disposition, Section 8 rental assistance programs, establishing a secondary marke for EEM securities, low-income weatherization programs, etc.); and

- Working with the HERS Council to track the impact of energy savings on loan security and valuation. 


\section{Implementation TAC}

Ohjective: Facilitate implementation of a national HERS/EEM program hy assisting in devising an implementation strategy for addressing:

- Infrastructure capable of national implementation and ongoing administration; and

- Initial funding.

\section{HERS TAC}

Composed of DOE representatives, HERS tool developers, HERS representatives, and shelter industry representatives who can assist in addressing technical issues of analysis tools and the process of rating houses.

Ohjective: Technical integrity and consistency of HERS programs and analysis tools hy:

- Approving analysis tools;

- Assisting in resolving technical issues such as integration of new technologies, assumptions (i.e., energy use of lights and appliances, hot tubs, etc.), new control strategies, off-peak energy use, quality control, etc.; and

- Serving as liaison to other industry groups such as American Society of Heating, Refrigerating, and Air-Conditioning Engineers, Fenestration Council, NAHB, Energy Efficient Builders Association, etc.

\section{HERS Council}

This would be a nonprofit membership organization. HERS Council members would include both approved scaled and certification program. The HERS Council could initially be umbrellaed by ERHA, with the option of heing spun off later as an independent, not-for-profit organization.

Ohjective: Implement, administer, and quality control a national HERS/EEM program to:

- Serve as a national clearinghouse of HERS/EEM information;

- Coordinate approval process for scaled HERS and certification programs for use with EEMs;

- Maintain a current list of approved HERS analysis tools and HERS programs for the mortgage industry and for states that are starting up programs;

- Provide one-point linkage hetween HERS programs and the secondary market, HUD/FHA, VA, FmHA, etc., and

- Provide technical assistance to states and jurisdictions for local HERS/EEM program development.

\section{AEEMSCO}

Association of EEM service companies could provide:

- Coordination of EEMs at time of sale by requesting ratings, evaluating improvement measures, ohtaining contractor hids, and oversecing installation of measures; and

- Consultation and training with shelter industries and local and state agencies in designing HERS/EEM programs. 


\section{Scaled HERS members}

This would be composed of state and local program entities that administer a scaled rating system-typically employed by states and certain jurisdictions for comparing all house types and sometimes used for alternative code compliance. These programs would:

- Employ an approved analysis tool;

- Be certified through a national or state umbrella organization member of the HERS Council; or

- Be certified directly by the HERS Council.

\section{Certification HERS members}

This includes certification programs typically employed by utilities, manufacturers, home builders, etc., which certify a house (typically new) to meet a certain standard of efficiency. These member programs would:

- Employ an approved rating tool;

- Be certified through a national or state umbrella organization member of the HERS Council; and

- Be certified directly by the HERS Council.

\section{HERS Council Associate Members}

This would be composed of national, regional, and local companies and organizations that would benefit from the success of a HERS/EEM program.

\section{Housing Data Bank}

A national data bank of energy characteristics of houses financed with EEMs would be capable of the following.

- Including efficiency levels of components, fuel source, estimated energy costs, metered costs, elc., of:

- all houses rated under approved HERS programs, and

- houses financed with EEMs using an approved analysis tool.

- Providing reports such as:

- correlation of cost (or savings) estimates compared to metered data for quality control;

- high, low, average, and baseline energy costs of different house types with different fuel mixes in different parts of the country;

- typical energy characteristics of housing stock in different regions;

- savings that have actually resulted from energy improvements in EEM programs;

- relative energy costs of houses in default (is there a high correlation?);

- tracking of EEM properties and defaults (is there a low correlation?); and

- correlation of efficiency and value in the appraisal process. 
Attachment to Special Paper No. 9

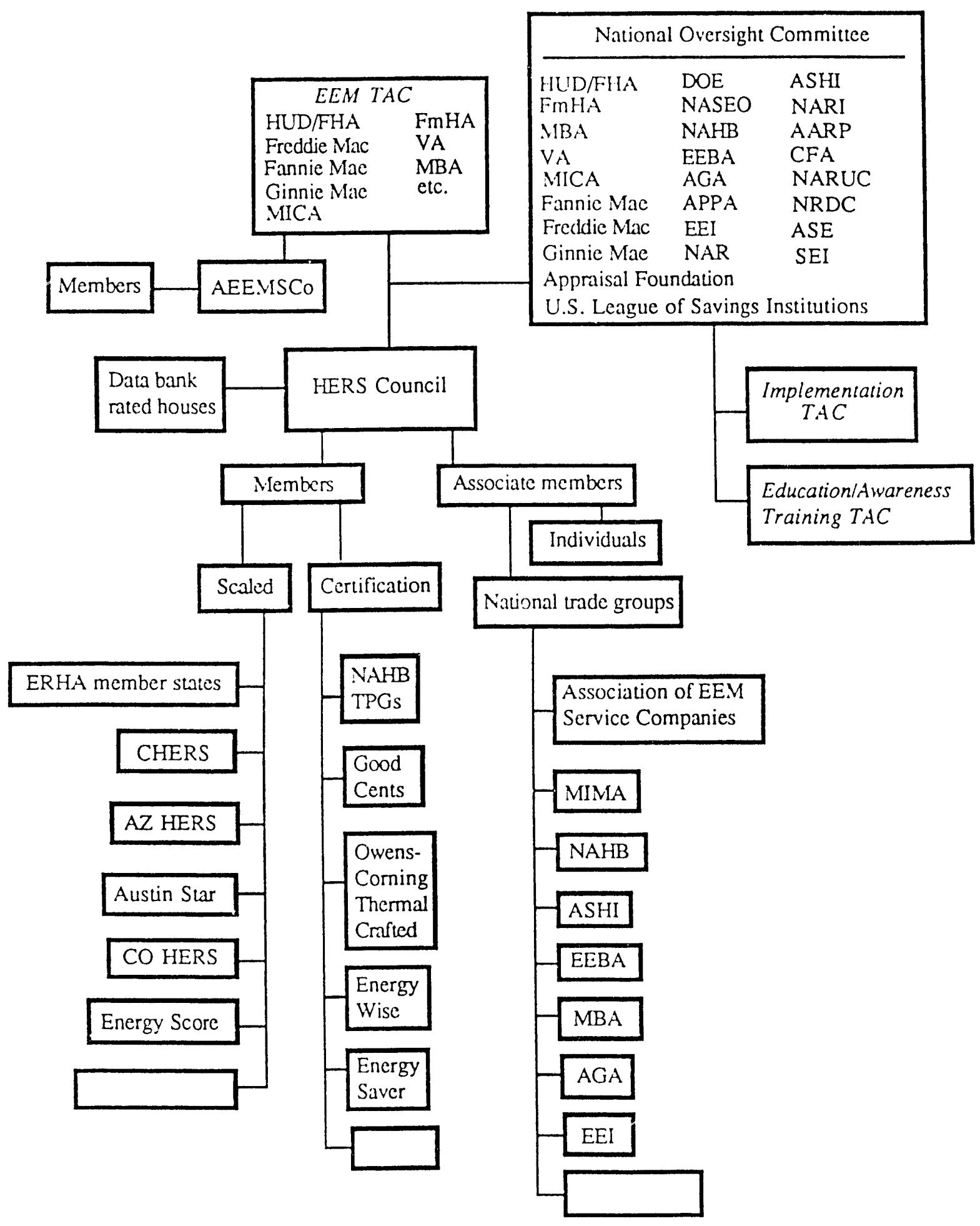

PROPOSED NATIONAL

HERS INFRASTRUCTURE 
Special Paper No.: 10

Contact person: Bill Prindle, Alliance to Save Energy ${ }^{11}$

Date of issue paper: Octoher 28,1991

Title of issue: $\quad$ Cash flow vs. cost-effectiveness

Recommendations (if any) (include majority/minority): Retrofits financed in an energy rated mortgag (ERM) should be both cost-effective and show positive cash flow to the homeowner. This dual analysi should be allowed as a uniform valuation method for energy retrofits in an ERM.

Summary of analyses already completed: By the most accepted methods used today, cost-effectiv means that an investment has a stream of benefits greater than or equal to its cost. For energy retrofil that means that the present (discounted) value of energy savings over the life of the measures must equ: or exceed installed cost. For example: if a package of retrofits costs $\$ 3000$ and saves $\$ 250$ per year, th present value of savings at a $7 \%$ discount rate over 30 years is $\$ 3102$; because the present value of saving is greater than the installed cost, this investment is cost-effective. The key variables here are the discour rate and the period over which savings are accrued (the service life of the equipment).

Positive cash flow means that the financed cost of the measures in a given payment period is less tha the savings in that period. For example, if the same $\$ 3000$ package of retrofits were financed in a 30 -ye: mortgage at $9 \%$ interest, the added mortgage payment would be $\$ 24.13$. The monthly energy saving would average $\$ 20.83$. Because the savings does not exceed the added payment, this investment does no produce positive cash flow. The key variables here are the intercst rate and term of the mortgage.

To extend this example a bit further, the "break-even" point for cost-effectiveness is at about $\$ 240 / y e a$ in savings. At this point the present value of savings equals the installed cost. By contrast, the break even point for cash flow is at $\$ 290$ /year in savings; at this point the added payment about equals th monthly energy savings.

Cost-effectiveness is the appropriate test of whether an investment is beneficial to society. Cash flow $\mathrm{i}$ an appropriate test of whether the investment directly benefits the individual consumer. In the abov example, note that with the terms of both analyses set at 30 years, the key variables hecome discount rat versus interest rate. Keeping a constant societal discount rate of $7 \%$ provides a stable indicator of th value of the retrofits to society. Using variations in interest rates to drive the cash flow test allows th effect of market factors to be applied and ensures that a given individual benefits economically.

Using simple cash flow tests in combination with cost-effectiveness tests provides a rigorous basis fo assessing the value of energy retrofits and may help solve the appraisal problem for ERM retrofits.

\footnotetext{
${ }^{11}$ Currently with Barakat and Chamberlin, Washington, DC.
} 
Special Paper No.: 11

Contact person: Bill Prindle, Alliance to Save Energy ${ }^{12}$

Date of issue paper: October 18, 1991

Title of issue: $\quad$ Key barriers to the wider use of EEMs for retrofitting existing homes

"Risk, Value, and Delay"

Increased perceived risk-For marginal loan applications, those at or near the maximum loan-to-value (LTV) ratios or maximum loan limits, adding to the loan amount creates the perception of a riskier loan, partly because of uncertainty about the value that energy efficiency features add to the home. This makes lenders uneasy about processing EEMs for marginal borrowers.

Appraisal/valuation-Currently, appraisers are asked to make judgements on the value of energy efficiency features based either on market comparables or an alternative present-value-of-savings method. Appraisers are very uncomfortable with this approach; thus there is no practical way to easily and consistently assign value to energy features added in an EEM. In addition, current rules often prohibit the addition of the full cost of energy improvements to the appraised value of the home. The appraisal issue must be simplified such that it takes the responsibility out of the appraiser's hands and allows the full cost of energy improvements (that pass economic and financial tests) to be added to the loan amount. This solution also must be coupled to the LTV/risk issue so that it does not create perceived risk to the mortgage industry.

Delay and complexity-Many EEM program rules can create delay and undue paperwork for professionals in the home sales, finance, and closing process:

First, most programs don't trigger borrower awareness early enough for the borrower to undertake the needed steps.

Second, most rules don't allow EEM paperwork to be added to the loan approval process late enough.

Third, underwriters and appraisers must typically re-underwrite and re-appraise the loan after EEM paperwork has been produced.

Fourth, the escrow arrangement for retrofits bothers many lenders, who, even if the loan can he shipped prior to completion of improvements, feel they are at risk if something goes awry between buyer and installer.

A suggested integrated solution to reducing these barriers follows.

\section{A Proposed Approach to Reducing the Three Key EEM Barriers}

To make EEMs work for retrofits, these interwoven issues-risk, value, and delay-must he addressed in a comprehensive way. I suggest one such solution helow in hroad terms.

\footnotetext{
${ }^{12}$ Currently with Barakat and Chamberlin, Washington, DC.
} 
Risk-At the least, restrict the LTV and maximum loan amounts for EEMs (o) current limits. In practice, it is my view that the most attractive segment of the market is horrowers bringing substantial equity to the transaction. The EEM is less appropriate for a first-time huyer than a trade-up huyer who has more equity in the transaction, is a less risky horrower, and has the experience with the closing process 10 he able to take on the added wrinkle of the EEM. Trying 10 push the limits of current mortgage rules in today's terrible real estate market could kill any chance of the EEM concept laking hold in the marketplace by causing it to be perceived as a risk-increasing program. Exceptions may he required in a few of the nation's highest-priced markets where median housing prices exceed FHA and conforming loan limits.

Value-Appraisers should he exempted from having to assign value to energy improvements. It is clear that they are unwilling to take on the task using anything other than traditional comparahle-market-value comparisons. The EEM programs should adopt a more uniform, calculation-hased approach for valuing improvements. This would be hased on variants of the current FmHA and Freddie Mall Form 7()-A procedures. I would suggest a two-step approach:

1. Require EEM improvements to meet a cost-effectiveness test. I would suggest a modified version of the FmHA "value-in-use" concept as the hasis for this. As I envision it, the present value of energy savings would have to equal or exceed installed cost, hased on the actual expected life of the measures and a 7\% discount rate. This relaxes the current 70-A formula, which limits equipment life to seven years and uses the mortgage interest rate for the discount rate. The rationale for the current formula is that the average buyer only stays in the house seven years, and the mortgage rate is a good proxy for a market-based discount rate. However, if the second test helow-positive cash flow-is met, there is no need to hold the energy improvements to a market-hased cost-effectiveness test. If the consumer enjoys positive cash flow, he is (in the terms of economics) indifferent to discount rates and related parameters. Thus the proper economic test for cost-effectiveness hecomes a societal test. Hence it is appropriate to use a societal discount rate $(7 \%)$ and the full life of the equipment rather than the buyer's average tenure.

2. Require a proposed package of energy retrofits to show positive cash flow to the homeowner. This means that the monthly energy savings from the retrofits must he greater than the added mortgage payment they would engender. In the case of an adjustable rate mortgage (ARM), graduated payment mortgage, or other mortgage where future payments may vary, energy savings musi exceed the maximum payment under the terms of the mortgage. For example, if an ARM has an opening rate of $7 \%$ and a cap of $12 \%$, the EEM measures would have to show positive cash flow at the $12 \%$ rate.

If a package of retrofits passes hoth these tests, 10()\% of its costs should he allowed to he added to the mortgage and to the appraised value of the home. The total cost of the retrofit package must not exceed a preset limit (say 5\% of the purchase price, with minimum and maximum dollar amounts, say $\$ 4()())$ and $\$ 1(),()()$, to he adjusted periodically). And the total "improved" Ioan amount must not exceed standard LTV and loan amount caps.

This kind of formula should satisfy all parties as to limiting risk and providing adequate analytical rigor to justify the $100 \%$-of-cost funding and valuation approach.

Delay - A key here is to trigger awareness, interest, and action as early as possible. This is mainly an awareness and promotion issue, hut it could he helped if there were routine information transfer points, such as the time of loan application or contract signing, when buyers were specifically told about their EEM options. A second, more central technical jssue is how to avoid having to re-underwrite and re-appraise the loan after the EEM data have heen produced. If the appraisal/LTV procedures outlined ahove are used, the underwriter and appraiser can be excused from a second look at the loan file. 
Someone would have to verify that the analysis was done correctly for eosteffectiveness and cast flow - probably the underwriter-but that would be a separate step from the hatsic underwriting and shumbl not slow down the process. The third problem is to get around the escrow hang-up. The primbiry lender needs to he removed from the poten ial liability and hassle of instiller error, negligence, hankruptey, and so on, not to mention huyer intransigence. Perhaps some sort of third party involvement for insurance or guarantee purposes would help. 
Special Paper No.: 12

Contact person: $\quad$ Stephen S. Szoke, Passive Solar Industries Council

Title of issue: $\quad$ Renewable energy technologies for home energy rating systems and energyefficient mortgages

\section{Introduction}

Renewable energy technologies produce marketable energy hy converting natural phenomena into useful forms of energy. Economical strategies exist to use the energy inherent in sunlight for operating buildings and providing human comfort. These sources of direct and indirect sunlight may be used to significantly reduce the need of fossil fuel sources of energy to provide light, heat, cooling, and hot water for residential buildings.

Broadly speaking, these technologies, using direct and indirect sunlight, include hydroclectric electric power generation, ocean energy, wind power, biofuels, and solar energy. This paper is limited to discussions of systems that may be economically incorporated into home energy rating systemis (HERS) and energy-efficient mortgages (EEMs). These technologies include:

- Passive solar space heating, cooling, and lighting

- Active solar space heating and cooling

- Solar (active and passive) water heating

- Photovoltaic generation of electricity

- Biofuel (wood burning) appliances

- Wind power generation of electricity.

Each of these components have a potential to significantly reduce the use of purchased energy for operating and maintaining comfort in residential buildings. Some of these technologies are more easily and accurately evaluated than others. In addition, some of these systems are more applicable to HERS or EEMs or both, than others.

\section{Passive solar heating, cooling, and lighting}

Passive solar design strategies are a combination of energy conservation and efficiency strategies and architectural design of the structure to effectively utilize the benefits of direct solar radiation incidence on the building. An estimated $3(0),(0)()$ homes in the United States employ some passive solar design features. The most popular systems include:

- Direct gain spaces

- Thermal storage wall systems

- Sunspaces

- Natural ventilation

- Sun tempering

- Thermal mass

- Daylighting.

These systems, and combinations of these systems, may practically and economically replace $30 \%$ of the primary energy used to heat, cool, and light residential huildings throughout the United States. In addition, in areas of high solar radiation incidence, the replacement of primary energy for heating and cooiing may economically exceed 7()$\%$. 
Each of these strategies has a varying degree of effect on heating, cooling, and lighting loads, depending on climate and building type. Daylighting by itself, for example, will have little impact in most singlefamily structures but could have a signilicant impact in multifamily structures. Daylighting in combination with sun tempering and direct gain strategies could he very effective. Sunspaces, direct gain strategies, and sun tempering are appropriate for both new and existing construction. Natural ventilation and thermal mass strategies are effective primarily as cooling strategies, while sun tempering, direct gain systems, thermal storage wall systems, and sunspaces are primarily effective as heating strategies.

Sun tempering is adding additional south facing glass in an amount not to exceed the storage capabilities of typical light frame construction. When glass is added to slab-on-grade or other high mass construction, the amount of glass and heat gain within acceptable comfort ranges is increased, thereby further reducing the amounts of purchased energy used for heating. Properly exposing the slab or other massive building components results in direct gain strategies. Sunspaces are direct gain strategies isolated from the main living spaces. In such spaces, temperatures may be permitted to fluctuate above normal acceptable comfort ranges. Thermal storage walls are storage materials, typically mass walls, placed immediately behind exterior glazings. These tend to provide the least interior temperature fluctuations. A good design may use combinations of these strategies.

Passive solar cooling strategies are typically natural ventilation driven by a temperature gradient within the structure as well as wind. The thermal mass strategies are used in combination with the mechanical system. Mass is cooled at night when system efficiencies are better and in many instances, rates are lower. In Phoenix, Arizona, the Salt River Project demunstrated that 97\% to 99\% of the cooling load may be shifted to off-peak hours with appropriately sized mass. Although thermal mass benefits for cooling may be documented, the use of natural ventilation as a cooling strategy may not be as appropriate for HERS and EEMs because of the sensitivities to climate conditions and building operation.

Passive solar energy design strategies previde reductions in primary energy use. All passive solar strategies with adequate storage componeni: level out and shift the time of the maximum loads. This thermal storage capability shifts energy demand in off-peak demand periods. This is an additional benefit because buildings with good passive solar design st ategies may permit utilities to use power generation facilities more efficiently. These significant benefits should be included as practical and economical options for any HERS or EEMs.

\section{Home energy rating systems}

The contribution of passive solar design strategies is not easily predicted by checklist or extremely' simplified calculation procedures used to certify building compliance to most codes and standards in place today. The effects of passive solar design are slightly more complex than steady-state heat loss and heat gain calculations typically used for compliance to energy codes. Methods for accurately predicting the potential energy savings resulting from passive solar heating strategies are provided in the American Society of Heating, Refrigerating and Air-Conditioning Engineers' Passive Solar Heating, A Design Manual. These procedures have been simplified for regional applications by several existing and ongoing programs of the Passive Solar Industries Council and the National Renewable Energy Laboratory under U.S. Department of Energy contracts.

Most computer programs used as research and analysis tools are sophisticated enough to account for the benefits of passive solar heating and cooling. Work continues for integrating daylighting henefits into these research and analysis tools. With the current state of the art, passive solar heating and cooling strategies may easily he incorporated into any HERS. Daylighting should he provided for whenever sophisticated tonk are used to rate huildings. Provisions should he available to include daylighting in HERS as the technology develons 
Simplified tools currently exist and new tools are being developed to analyze and design passive solar buildings. Passive Solar Design Strategies: Guidelines for Home Builders and the accompanying Builder Guide computer program are such tools. Retrofit guidelines are being finalized, and tools for determining the amount and time of peak loads are being developed.

Any HERS would be remiss if it did not have provisions that not only account for, but encourage, the use of passive solar heating, cooling, and lighting. In residential buildings, optimal energy efficiency may only be achieved with an appropriate balance of conservation, efficiency, and passive solar technologies.

\section{Energy-efficient mortgages}

Passive solar technologies not only provide the benefit of reducing primary energy use, but also tend to shift the time of primary energy use to off-peak hours. Thus, passive solar strategies that have the ability to provide reductions in primary energy use that far exceed the reductions economically achieved by conservation of efficiencies alone also have the benefit of using certain primary energy resources at reduced (time of use) rates. The benefits are both reduced amount of purchased energy plus purchasing certain energy types at reduced rates. All EEMs must appropriately consider passive solar design strategies.

\section{Active solar space heating and cooling}

Active solar space conditioning systems are not currently providing a significant impact on energy requirements for maintain ng comfort in buildings. There is limited use of these systems; however, there is a significant potential icr growth with these heating and cooling strategies and technologies.

These systems tend to be such that they replace or supplement the components of conventional space conditioning equipment. These systems should easily be incorporated into any HERS or EEMs.

\section{$\underline{\text { Home energy rating systems }}$}

Active solar space conditioning systems are equipment added to the building to reduce the need for primary energy use. These systems are considerably different than passive solar design strategies, which are the architecture of buildings themselves. Active solar space heating and cooling systems should be included as primary or supplemental equipment for heating, cooling, or both in any HERS. These systems should be easily incorporated into the equipment provisions of HERS.

\section{Energy-efficient mortgages}

As previously mentioned, active solar technologies are essentially equipment, not the materials used to construct the building itself. Thus, like all equipment, the efficiencies, maintenance, and replacement schedules must be appropriately considered in any EEM, and the Solar Energy Industries Association

should be consulted. Active solar space conditioning equipment and systems should be included in any EEM program.

\section{Solar water heating}

Water for domestic use may be heated by active or passive solar systems. While the passive solar strategies for heating water exist, the main stream solar water heating technologies are active solar. Over a million active solar water heating systems have been installed in the United States hefore 1990. 
Active solar water heating systems should readily be inciuded in any HERS or EEM programs. Like active solar space conditioning systems, the water heating systems should be included in the appropriate equipment provisions. The Solar Energy Industries Association (SEIA) should be consulted for information on the appropriate methods of incorporating active solar water heating systems into HERS and EEMs.

$\underline{\text { Home energy rating systems }}$

Solar water heating systems should be included in all HERS. Solar water heating, particularly active solar water heating, may significantly reduce the use of primary energy in many residences. These systems are easily incorporated into a HERS program as equipment.

\section{Energy-efficient mortgages}

Solar water heating should be included in all EEMs. The reduction in primary energy use, reducing the need to purchase energy for domestic water heating, may be significant, especially for larger families.

\section{Photovoltaic electric generation}

The electricity generated by photovoltaic systems may be used to operate appliances and equipment in homes. This reduces the need for purchased primary energy. The benefits of photovoltaics are not easily accounted for in buildings, because benefits may be extremely dependent on building and appliance operation.

\section{$\underline{\text { Home energy rating systems }}$}

Since the benefits of photovoltaics are dependent on the habits of the building occupants, there are some difficulties in accurately predicting the performance or impact on primary energy use. Thus, there is some question as to the appropriateness for HERS. Based on the recommendations of the HERS Technical Advisory Committee (TAC), HERS should be based on standardized methods of building, appliance, and equipment operation. Thus, the benefits of photovoltaics could be easily and appropriately accounted for as an on-site energy source for reductions in use of primary energy.

\section{Energy-efficient mortgages}

Whether or not photovoltaics are found to be accurately incorporated into HERS, photovoltaic electric generation systems should be a consideration in any EEM. This may be appropriate, especially if certification for the EEM is based on actual purchased energy. Photovoltaics may better fit other strategies or programs developed by utilities, as rebate or other type programs, rather than being incorporated into HERS or EEMs.

\section{Wood burning appliances}

Wood burning appliances may be used to provide space heating, water heating, or preheat for other space and water heating systems. About five million households use wood as the primary space heating source, and another seventeen million use wood as a supplementary heating source. The wood is burned in either wood stoves or fireplaces. The energy conservation and efficiencies related to these systems are difficult to accurately predict. 


\section{Home energy rating systems}

The benefits and reduction in primary energy use are difficult to determine for most wood burning stoves and fireplaces unless the wood hurning appliance is the sole source of heating. For these instances, HERS should have provisions to include wood burning stoves and fireplaces used for space or water heating. These provisions might be best incorporated into the equipment section of a HERS evaluation.

\section{Energy-efficient mortgages}

Wood burning appliances may be the primary source of heat and thus should be considered in any EEM. There is a difficulty in implementation of EEMs when wood burning appliances are used for supplementary heating of space or water. Efficiencies and effectiveness of wood burning appliances are extremely dependent on operation techniques. One operator may achieve significantly different performance than another operator. Thus, for most instances, wood burning appliances, especially when used for supplementary space or water heating, may not be appropriate for inclusion in EEMs.

In addition to the fact that one operator may have significantly lower primary energy use than another when wood burning appliances are used for supplementary heat, provisions are required to include the purchase price, if any, associated with the wood fuel. Wood burning appliances may only be adaptable to EEMs that are based on actual utility and wood purchases. The Wood Heating Alliance should be consulted for more details on the appropriateness of wood burning appliances for HERS and EEMs.

\section{Wind power}

Electric generation for domestic use by wind power is similar in relationship to HERS and EEMs as photovoltaic electric power generation. Wind power generated electricity may be more appropriate for programs other than HERS or EEMs.

\section{Home energy rating systems}

Electric power generation is difficult to accurately predict because of the dependency on the wind, but like with all predictions for HERS, standardized weather and occupant conditions are assumed. Thus, the generation of electricity used to operate appliances and equipment is appropriate for inclusion in HERS.

\section{Energy-efficient mortgages}

Whether or not wind power for electric gerieration is found to be appropriately considered in HERS, these systems may be a consideration for EEMs, especially if the EEM is based on actual purchased energy.

\section{Summary}

Each of the systems discussed may have a significant impact on the cost of operating and maintaining comfort in a residential structure. Based on the current state-of-the-art in the building sector, appropriateness for HERS and EEMs, and impact on overall purchased energy use, each of these renewable energy strategies for residential huildings are categorized for level of consideration in HERS and EEMs. The categories are $\mathrm{H}$ for high, $\mathrm{M}$ for moderate, and $\mathrm{L}$ for low. The strategies that ranked high should be included in HERS and EEMs. These rankings are hased on the discussions that occurred at the HERS Technical Advisory Committee meetings. 


\begin{tabular}{||l|c|c||}
\hline \multicolumn{1}{|c|}{ Strategy } & HERS & EEMs \\
\hline \hline Passive Solar Space Heating & $\mathrm{H}$ & $\mathrm{H}$ \\
Cooling and Lighting & & \\
Sun Tempering & $\mathrm{h}$ & $\mathrm{h}$ \\
Direct Gain Systems & $\mathrm{h}$ & $\mathrm{h}$ \\
Thermal Storage Wall Systems & $\mathrm{h}$ & $\mathrm{h}$ \\
Sunspaces & $\mathrm{h}$ & $\mathrm{h}$ \\
Natural Ventilation & $\mathrm{l}$ & $\mathrm{h}$ \\
Thermal Mass & $\mathrm{h}$ & $\mathrm{h}$ \\
Daylighting & $\mathrm{l}$ & $\mathrm{m}$ \\
\hline Active Solar Space Heating & $\mathrm{H}$ & $\mathrm{H}$ \\
and Cooling & & $\mathrm{H}$ \\
\hline Solar Water Heating & $\mathrm{H}$ & $\mathrm{h}$ \\
Active Systems & $\mathrm{h}$ & $\mathrm{m}$ \\
Passive Systems & $\mathrm{m}$ & $\mathrm{M}$ \\
\hline Photovoltaic Generation of & $\mathrm{L}$ & $\mathrm{m}$ \\
Electricity & $\mathrm{L}$ & $\mathrm{M}$ \\
\hline Wood Burning Appliances & $\mathrm{L}$ & $\mathrm{M}$ \\
Primary Heating Source & $\mathrm{h}$ & $\mathrm{h}$ \\
Supplementary Heating Source & $\mathrm{L}$ & $\mathrm{m}$ \\
\hline Wind Power Generation of & & \\
Electricity & & \\
\hline
\end{tabular}

It is essential that any HERS or EEM program include as many provisions for the use of renewable energy strategies as possible. In addition, for the benefits intended by the implementation of HERS and EEMs, each program should not only include but encourage the use of renewable energy sources. Programs based solely on conservation and efficiency strategies for primary energy use reductions are not sufficiently adequate for the long term needs of the nation and in many instances, do not provide sufficient reductions in energy use to be of benefit to evaluating mortgage qualifications.

Conservation and efficiency programs are more easily implemented than the more complex aspects of many strategies that use renewable energy sources. Because of the many long term benefits of renewable energy sources for operating and maintaining comfort in residential structures, dependency on simplistic, easy to implement approaches should not be the highest priority. Instead, if complexity is a barrier, then education and development of simplified tools are the solutions. These important aspects of building design and equipment cannot afford to he neglected based on ease of implementation. Renewable energy strategies must he encouraged hy any HERS or EEM at least equally and preferably more than conservation and efficiency strategies alone. 


\section{References}

The Potential of Renewable Energy - An Inter-laboratory White Paper, Idaho National Engincering Laboratory, Los Alamos National Laboratory, Oak Ridge National Laboratory, Sandia National Laboratories, Solar Energy Research Institute. Prepared for the Office of Policy, Planning and Analysis, U.S. Department of Energy under contract No. DE-ACO283CH10093. Published by Solar Energy Research Institute, a Division of Midwest Research, 1617 Cole Boulevard, Golden, CO 80401-3393. 
Special Paper No.: 13

Contact person: Marika Tatsutani, National Resources Defense Council

Date of issue paper: December 5, 1991

Title of issue: $\quad$ Environmental benefits of a national HERS/EEMs program

Among the most important beneficiaries of a successful home (energy-efficient mortgage) program will be the environment. By reducing the amount of energy required to heat, cool, and power thousands of homes, pollution that would otherwise contribute to acid rain, global warming, urhan air quality problems, and land and water degradation can be avoided. By contributing to a healthier environment for all Americans, the potential benefits of a (HERS/EEMs) program will extend well beyond those homeowners and home buyers who participate directly.

Currently, the residential sector accounts for some $16 \%$ of the nation's total end-use energy consumption or over 10 quadrillion Btu every year: the equivalent of 368 million metric tons of coal or 1.8 billion barrels of oil. ${ }^{13}$ The consumption of this enormous quantity of energy-largely from fossil fuel resources-by the residential sector alone, is proportionately responsible for the more than 5.2 billion tons of carbon dioxide, 19.8 million tons of nitrogen oxides, and 23.1 million tons of sulfur oxides dumped into the global atmosphere by U.S. homes, automobiles, businesses, and industries every year. ${ }^{14}$

Indeed, the "hidden" environmental costs of inadequately weatherized houses or inefficient heating systems range from oil befouled beaches to the acidification of fragile aquatic ecosystems in our most pristine wilderness areas. Global warming, in particular, is a direct environmental consequence of fossil fuel consumption that is likely to prove especially troublesome in the decades to come. Scientists now predict that the continued addition of carbon dioxide and other so-called "greenhouse gases" to the atmosphere will, if current emissions patterns persist, result in global mean temperature rise at the rate of $0.2^{\circ}$ to $0.5^{\circ} \mathrm{C}$ per decade during the next century. This is a rate of temperature increase unprecedented in the last 10,000 years and could result in an earth that is, on the whole, several degrees hotter by some time in the next 50 years. ${ }^{15}$ The implications of such a scenario are potentially devastating in economic, environmental, and human terms. Sea level rise would threaten coastal and low-lying areas worldwide, shifting regional climatic patterns would disrupt agricultural production and jeopardizc world food supplies, and ecosystem damage and species extinction would accelerate to frightening new levels.

By improving the efficiency of energy use in its residential housing stock, and in other sectors of the economy, the U.S. can take a significant step toward stabilizing and eventually reducing its greenhouse gas emissions, which, at present, contribute disproportionately to global climate change. The technology exists now to achieve total energy savings ranging from $50 \%$ to as much as $80 \%$ in home retrofits and

\footnotetext{
${ }^{13}$ Alliance to Save Energy, American Council for an Energy-Efficient Economy, Natural Resources Defense

Council, and Union of Concerned Scientists. 1991. America's Energy Choices: Investing in a Strong Economy and a Clean Environment. Cambridge, MA: Union of Concerned Scientists.

${ }^{14}$ Ibid.

${ }^{15}$ Lashof, Daniel A., and Dennis A. Tirpak, eds. Decemher 1990. Policy Options for Stabilizing Global Climate. Washington, DC: U.S. EPA.
} 
new construction. ${ }^{16}$ A mortgage program that helps tap the enormous potential for cost-effective energy savings makes good sense, not only for today's homeowners, but to ensure the health of the environment for future generations.

${ }^{16}$ Alliance to Save Energy, et al., 1991. op cit. 


\section{Appendix A \\ Members of the National Collaborative}

Listed are members of the Collaborative Consensus Committee (CCC), members of the Collaborative Technical Advisory Committees, members of the CCC's Steering Committee, staff, and official observers.

\section{Collaborative Consensus Committee (CCC) Members}

Alliance to Save Energy (ASE) - Bill Prindle (Member), Washington, DC

American Association for Retired Persons (AARP) - George Gaberlavage (Member), Washington, DC;

Jo Reed (Alternate), Washington, DC

American Gas Association (AGA) - Alan Hudenets (Member), Arlington, VA; Mary Carson (Alternate), Arlington, VA

American Public Power Association (APPA) - Barry Moline (Member), Washington, DC; Doug Swartz (Alternate), Fort Collins, $\mathrm{CO}$

American Society of Home Inspectors (ASHI) - Michael Lennon (Member), Falls Church, VA

Appraisal Institute (AI) - Steve Azia (Member), Washington, DC

Association of Energy Efficient Mortgage Service Companies (AEEMSC) - Jim Curtis (Member), Palo Alto, CA

California Home Energy Rating System, Inc. (CHERS) - Jim Fulkerson (Member), San Francisco, CA

Consumer Federation of America (CFA) - David Swankin (Member), Washington, DC; Rebecca Cohen (Alternate), Washington, DC

Edison Electric Institute (EEI) - Tom Farkas (Member), Washington, DC

Energy Efficient Builders Association (EEBA) - Don Buchan (Member), Ottawa, Ontario, Canada

Energy Rated Homes of America (ERHA) - Ron Hughes (Member), Little Rock, AR

Farmers Home Administration (FmHA) - Rich Davis (Member), Washington, DC

Federal Home Loan Mortgage Corporation (Freddie Mac) - Charles Fritts (Member), Washington, DC; Jane Stockinger (Alternate), McLean, VA

Federal National Mortgage Association (FNMA - Fannie Mae) - Chip Coffay (Member), Washington, DC

Federal Housing Administration (FHA) - Mark Holman (Co-Member), Washington, DC; Morris "Bud" Carter, (Co-Member), Washington, DC

Federal Institutions Examination Council, Appraisal Suhcommittee - Ted Baker (Member), Washington, DC

Mortgage Bankers Association (MBA) - Bill Brewster (Memher), Washington, DC

National Association of Home Builders (NAHB) - Perry Bigelow (Co-Member), Palatine, IL; Charles Field (Co-Memher), Washington, DC

National Association of the Remodeling Industry (NARI) - Patti Knoff-Burgio (Memher), Arlington, VA

National Association of REALTORS TM (NAR), Government Relations Division - Lee White (Member), Washington, DC; George Griffin (Alternate), Washington, DC

National Association of State Energy Officials (NASEO) - Steve Baden (Co-memher), Anchorage, AK; Doug Culbreth (Co-memher), Raleigh, NC; Chris Roitsch (Co-memher), Austin, TX

Natural Resources Defense Council (NRDC) - Dan Lashof (Memher), Washington, DC; Marika Tatsutani (Alternate), Washington, DC

Southern Electric International, Good Cents Division Binny Folsom (Memher), Allanta, GA

U.S. Department of Veterans Affairs (DVA) - Rohert Finneran (Memher), Springfield, VA 
Technical Advisory Committee (TAC) Members

\section{Home Energy Rating Systems (HERS) TAC}

CCC Co-Chair: Bill Prindle, Alliance to Save Energy, Washington, DC (now with Barakat and Chamberlin)

CCC Vice Chair: $\quad$ Steve Baden, National Association of State Energy Officials, Anchorage, AK

Staff Co-Chair: $\quad$ Ron Judkoff, National Renewable Energy Laboratory, Golden, CO

Members:

Tim Butler, Energy Division, North Carolina Department of Economic and Community Development, Raleigh, NC

William Freeborne, Policy Development and Research, U.S. Department of Housing and Urban Development, Washington, DC

Michael Holtz, Architectural Energy Corporation, Boulder, CO

Bion Howard, Alliance to Save Energy, Washington, DC

Steve Kennedy, Georgia Power, Atlanta, GA

Ron Nickson, National Association of Home Builders, Washington, DC

Doug Swartz, Light and Power Utility, City of Fort Collins (Co-member with IMP TAC), Fort Collins, CO

Steve Szoke, Passive Solar Industries Council, Herndon, VA

\section{Energy-Efficient Mortgages (EEMs) TAC}

CCC Co-Chair: Bill Brewster, Mortgage Bankers Association, Washington, DC

Staff Co-Chair: $\quad$ Ken Crandall, Federal Housing Administration, Washington, DC

Members:

Richard Faesy, Energy Rated Homes of Vermont, Burlington, VT

Matt Felber, Farmers Home Administration, Washington, DC

Walter Patterson, Texas Energy Extension Service, Arlington, TX

William Sorrentino, National Association of Home Builders, Washington, DC

Jane Stockinger, Federal Home Loan Mortgage Corporation (Freddie Mac), McLean, VA

\section{Implementation TAC}

CCC Co-Chair: Charles Field, National Association of Home Builders, Washington, DC

Staff Co-Chair: $\quad$ Rebecca Cohen, Consumer Federation of America, Washington, DC

Members:

David Dacquisto, National Association of Home Builders, National Research Center, Upper Marlhoro, MD Russell Duncan, Carolina Power and Light, Raleigh, NC

Javier Figueroa, Washington State Energy Office, Olympia, WA

Stuart Harshbarger, Washington Gas Light, Washington, DC

Doug Seiter, City of Austin, Austin, TX

Doug Swartz, Light and Power Utility, City of Fort Collins (Co-memher with HERS TAC), Fort Collins, $\mathrm{CO}$

Richard Tracey, Ryland Homes, Columbia, MD 


\section{Awareness TAC}

CCC Co-Chair: $\quad$ David Swankin, Consumer Federation of America, Washington, DC

Staff Co-Chair: $\quad$ Gerry McGowan, Philadelphia Department of Energy Support Office, Philadelphia, PA

Members:

William Griffin, Commonwealth Edison, Chicago, IL

Deborah Howard, Arizona Energy Office, Tucson, AZ

Randy Martin, Iowa Department of Natural Resources, Des Moines, IA

Rebecca Vories, Infinite Energy, Denver, CO

\section{Staff}

U.S. Department of Energy (DOE)

John P. Millhone, Deputy Assistant Secretary, Office of Building Technologies, Washington, DC Mary-Margaret Jenior, Program Manager, Office of Building Technologics, Washington, DC Gerry McGowan, DOE Philadelphia Support Office, Philadelphia, PA

National Renewable Energy Laboratory (NREL)

Barbara C. Farhar, HERS/EEM Program Leader, Collaborative Co-Facilitator, Washington, DC Ron Judkoff, Staff Co-Chair, HERS TAC, Golden, CO

Dorian McDuffie, Alyssa Tonelli, Kim Rattley, Conference Arrangements, Washington, DC Lauren Raphael, Technical Writer, Washington, DC

Mary Anne Dunlap, Technical Editor, Golden, CO

Princeton Economic Research, Inc. (PERI)

Joseph M. Cohen, Minutes, Rockville, MD

U.S. Department of Housing and Urban Development (HUD)

Energy Division: Bob Groberg, Lois Dean, Observers, Washington, DC

Federal Housing Administration (FHA): Ken Crandall, Staff Co-Chair EEMs TAC, Washington, DC

Facilitator: John A.S. McGlennon, ERM-New England, Inc., Boston, MA

\section{Steering Committee Members}

Steve Baden, National Association of State Energy Officials, Anchorage, AK

Bill Brewster, Mortgage Bankers Association, Washington, DC

Rebecca Cohen, Consumer Federation of America, Washington, DC

Ken Crandall, Federal Housing Administration, Washington, DC

Barbara Farhar, National Renewable Energy Laboratory, Washington, DC

Mary-Margaret Jenior, U.S. Department of Energy, Washington, DC

Ron Judkoff, National Renewable Energy Laboratory, Golden, CO

Gerry McGowan, U.S. Department of Energy, Philadelphia Support Office, Philadelphia, PA

John Millhone, U.S. Department of Energy, Washington, DC

Bill Prindle, Alliance to Save Energy, Washington, DC

David Swankin, Consumer Federation of America, Washington, DC 


\section{Official Observers}

American Gas Association - Paul Cabot, Jane Marder, Bruce Savage, Arlington, VA

Appraisal Foundation - - Dehorah Sharp, Washington, DC

Appraisal Institute - Don Kelly, Washington, DC

Arizona Energy Office - Charles Gohman, Phoenix, AZ

Air Conditioning and Refrigeration Institute - Richard Denny, Arlington, VA

California Energy Commission - John Wilson, Sacramento, CA

City of Manassas - Randy Bowers, Manassas, VA

Communications Consulting - Laura Braustcin, Reston, VA

Council of American Building Officials - Bob Spangler, Falls Church, VA

Electric Power Research Institute - Whitney Carroll, Michael Tinkleman, Washington, DC

Energy Efficient Builders Association - Bill Eich, Spirit Lake, IA

Energy Mines and Resources-Canada - Jack Cole, Ottawa, Ontario, Canada

Energy Rated Homes of America - Evan Brown, Dana Nixon, Little Rock, AR

Environmental Protection Agency - Adam Klinger, Washington, DC

Federal Home Loan Mortgage Corporation - Virgil Griffin, Washington, DC

Federal National Mortgage Association - John Nevin, Mark Simpson, Washington, DC

Florida Energy Office - Daryl O'Connor, Tallahassee, FL

Home Automation Association - Michacl Coffey, Eric Davidson, Nick Pyle, Washington, DC

Independent Bankers Association - Marti Sworobuk, Washington, DC

Mineral Insulation Manufacturers Association - George Phelps, Alexandria, VA

Maryland Building Industries Association - Frank Merditch, Landover, MD

Mortgage Insurance Companies of America - Frank Balser, Washington, DC

National Association of REALTORS ${ }^{\mathrm{TM}}$ - Deborah Lassman, Steve O'Connor, Washington, DC

National Association of Regulatory Utility Commissioners - Michael Foley, Washington, DC

National Institute of Standards and Technology, Heat Transfer Group - Fanney Hunter, Boh Zarr, Gaithershurg, MD

National Association of State Energy Officials - Frank Bishop, Washington, DC

National Energy Specialist Association - Frank Newbraugh, Topeka, KS

National Renewable Energy Laboratory - Ren Anderson, Golden, CO

National Resources Defense Council - Jennifer Jordan, Washington, DC

New York State Energy Office - David Abrey, Kevin O’Brien, Alhany, NY

Pennsylvania Electric - Steve McDonnell, Montrose, PA

RISE - Dehorah Curry, Providence, RI

The Society of the Plastics Industry, Inc. - George H. Sievert, Washington, DC

Southern California Edison - Gregg Ander, Rosemead, CA

U.S. Department of Energy - Ted Kapus, Sarah Kirchen, Simon Sidamon-Eristoff, Washington, DC

U.S. Department of Energy, New York Support Office - Joy Cazeaux, New York, NY

U.S. Department of Housing and Urhan Development - Andrew Euston, Dick Manuel, Heidi Martin, Roxanne Zimmerman, Washington, DC

U.S. Department of Veterans Affairs - Doug Widener, Washington, DC

U.S. Environmental Protection Agency - - Adam Klinger, Washington, DC

U.S. League of Savings Institutions - Daniclle French, Washington, DC

Vermont Housing Finance Agency - Doug Lothrop, Burlington, VT

Virginia Encrgy Office - J Jim Smith, Richmond, VA 


\section{Appendix B \\ Acronyms}

\begin{tabular}{|c|c|}
\hline AARP & American Association for Retired Persons \\
\hline A-EEM-SCOs & Association of Energy Efficient Mortgage Service Companies \\
\hline AFUE & annualized fuel utilization efficiency \\
\hline $\mathrm{AGA}$ & American Gas Association \\
\hline AI & The Appraisal Institute \\
\hline APPA & American Public Power Association \\
\hline ASE & Alliance to Save Energy \\
\hline ASHI & American Society of Home Inspectors \\
\hline ASHRAE & American Society of Heating, Refrigerating, and Air-Cunditioning Engineers \\
\hline $\mathrm{CABO}$ & Council of American Building Officials \\
\hline CCC & Collaborative Consensus Committee \\
\hline $\mathrm{CE}$ & Office of Conservation and Renewable Energy (within DOE) \\
\hline CFA & Consumer Federation of America \\
\hline CFR & Code of Federal Regulations \\
\hline CHERS, Inc. & California Home Energy Rating System \\
\hline COP & coefficient of performance (ratio of heat delivered to energy consumed) \\
\hline DOC & U.S. Department of Commerce \\
\hline DOE & U.S. Department of Energy \\
\hline DSM & demand-side management \\
\hline DVA & U.S. Department of Veterans Affairs \\
\hline EEBA & Energy Efficient Builders Association \\
\hline EEI & Edison Electric Institute \\
\hline EEMs & energy-efficient mortgages \\
\hline EER & energy-efficient ratio \\
\hline EMV & energy mortgage valuation \\
\hline EPA & U.S. Environmental Protection Agency \\
\hline EPRI & Electric Power Research Institute \\
\hline ERHA & Energy Rated Homes of America \\
\hline FHA & Federal Housing Authority \\
\hline FHLMC & Federal Home Loan Mortgage Company (Freddic Mac) \\
\hline FIEC & Federal Institutions Examination Council \\
\hline FmHA & Farmers Home Administration \\
\hline FNMA & Federal National Mortgage Association (Fannie Mae) \\
\hline FTC & Federal Trade Commission \\
\hline GNMA & Government National Mortgage Association \\
\hline GPO & Government Printing Office \\
\hline GRI & Gas Research Institute \\
\hline GSA & General Services Administration \\
\hline HBAs & Home Builder Associations \\
\hline HBI & Home Builders Institute \\
\hline HDD & heating degree days \\
\hline HERS & Home energy rating system(s) \\
\hline HUD & U.S. Department of Housing and Urban Development \\
\hline HVAC & heating, ventilating, and air conditioning \\
\hline IAQ & indoor air quality \\
\hline $\mathrm{kW}$ & kiliwall \\
\hline$k W h$ & kilowalt-hour \\
\hline
\end{tabular}




\begin{tabular}{|c|c|}
\hline LCC & life-cycle costing \\
\hline LTV & loan-to-value ratio \\
\hline MBA & Mortgage Bankers Association of America \\
\hline MCS & Model Conservation Standards \\
\hline MEC & Model Energy Code \\
\hline MHCSS & Manufactured Home Construction and Safety Standards (issued by HUD) \\
\hline $\operatorname{MIC} \Lambda$ & Mortgage Insurance Companies of America \\
\hline MIT & Massachusetts Institute of Technology \\
\hline MW & megawatt \\
\hline NAHB & National Association of Home Builders \\
\hline NAR & National Association of REALTORS ${ }^{\mathrm{TM}}$ \\
\hline NARI & National Association of the Remodeling Industry \\
\hline NASEO & National Association of State Energy Officials \\
\hline NCSL & National Council of State Legislatures \\
\hline NES & National Energy Strategy \\
\hline NESA & National Energy Specialist Association \\
\hline NGA & National Governor's Association \\
\hline NIST & National Institute of Standards and Technology \\
\hline NRDC & National Resources Defense Council \\
\hline NREL & $\begin{array}{l}\text { National Renewable Energy Laboratory (formerly the Solar Energy Research Institute - } \\
\text { SERI) }\end{array}$ \\
\hline OBT & Office of Building Technologies \\
\hline OPA & Office of Planning and Assessment (within DOE) \\
\hline OTFA & Office of Technical and Financial Assistance \\
\hline PITI & principal, interest, real estate taxes, and hazard insurance \\
\hline PSIC & Passive Solar Industries Council \\
\hline PUC & public utilities commission \\
\hline PUD & public utility district \\
\hline R\&D & research and development \\
\hline $\mathrm{SC}$ & Steering Committee of the National Collaborative \\
\hline SECP & State Energy Conservation Program \\
\hline SEER & seasonal energy efficiency rating (Btu/watt $\mathrm{hr}$ ) \\
\hline SEI & Southern Electric International \\
\hline SEO & state energy office \\
\hline SERI & the former Solar Energy Research Institute, see NREL \\
\hline TAC & Technical Advisory Committee of the National Collaborative \\
\hline TPG & Thermal Performance Guidelines \\
\hline
\end{tabular}




\section{Glossary}

Absorptance - The ratio of the radiation absorbed by a surface to the total energy falling on that surface described as a percentage.

Acquisition cost - In a HUD-FHA transaction, the price the borrower paid for the property plus any of the following costs: closing, repairs, or financing (except discounts in other than a refinance transaction) properly paid by the borrower. Does not include prepaid discounts in a purchase transaction, mortgage insurance premiums, etc.

Active solar energy system - A system designed to convert solar radiation into usable energy for space, water heating, or other uses. It requires a mechanical device, usually a pump or fan, to collect the sun's energy.

Addition - An alteration to an existing building that increases conditioned space.

Adjusted coefinicient of performance (ACOP) - A standard rating term that was used to rate the efficiency of heat pumps in California. ACOP was replaced by Heating Seasonal Performance Factor (HSPF) in 1988.

Affidavit - A sworn statement in writing before a proper official, usually a Notary Public.

Air change - The replacement of a quantity of air in a space within a given period of time, typically expressed as air changes per hour. If a building has one air change per hour, this is equivalent to all of the air in the building being replaced in a one-hour period.

Air conditioner - An assembly of equipment for air treatment consisting of a means for ventilation, air circulation, air cleaning, and heat transfer (either heating or cooling). The unit usually consists of an evaporator or cooling coil, and an electrically driven compressor and condenser combination.

Air film - A layer of still air adjacent to a surface, which provides some thermal resistance.

Air film coefficient - A measure of the heat transfer through an air film. (See American Society of Heating, Refrigerating, and Air-Conditioning Engineers [ASHRAE] Table 1, ASHRAE Handbook, 1985 Funclamentals.)

Air-to-air heat exchanger - A device with separate air chambers that transfers heat between the conditioned air being exhausted and the outside air being supplied to a building.

Alteration - Any change or modification to a building's construction. See Addition.

Ambient air temperature - Surrounding temperature, such as the outdoor air temperature around a building.

Amenity - An aspect of a property that enhances its value. Off-street reserved parking within a condominium community is an amenity.

American National Standards Institute (ANSI) A national organization that coordinates development and maintenance of consensus standards and sets rules for fairness in their development. ANSI also represents the United States in developing standards. 
Amortization - Gradual payoff of a debt through installment payments of principal and interest. Most modern mortgages amortize monthly over the term of the loan.

Angle of incidence - The angle that the sun's rays make with a line perpendicular to a surface. The angle of incidence determines the percentage of a direct sunshine intercepted hy a surface.

Annual energy cost savings - The difference in the first year cost of energy of the proposed or existing building or facility compared with the energy cost of its representative structure.

Annual fuel utilization efficiency (AFUE) - A measure of heating efficiency, in consistent units, determined by applying the federal test method for furnaces. This value is intended to represent the ratio of heat transferred to the conditioned space by the fuel energy supplied over one year. (See California Code of Regulations, Title 20, Section 1602[d][1].)

Annual operation and maintenance cost - The incremental cost to operate and maintain an energysaving measure compared with the operation and maintenance costs of its representative structure.

Appliance efficiency standards - Appliance efficiency standards establish the performance requirements for appliances sold in California. These standards apply to refrigerators, freezers, room air conditioners, central air conditioners, gas space heaters, water heaters, plumbing fittings, fluorescent lamp ballasts and luminaires, and ignition devices for gas cooking appliances, and gas pool heaters. New national appliance standards are in place for some of these appliances and will become effective for others at a future date. (See California Code of Regulations, Title 20, Chapter 2, Subchapter 4: Energy Conservation, Article 4: Appliance Efficiency Standards.)

Applicant - A prospective mortgage borrower.

Appraisal - A report made by a qualified person setting forth an opinion or estimate of value. The term also refers to the process by which this estimate is obtained. In conventional mortgages and in the HUD-FHA Direct Endorsement Program, the lender receives a copy of the complete report, showing the basis for the appraiser's estimate. In VA cases and in HUD applications processed by HUD, the lender receives only a statement of the estimate of value, without any detailed supporting data.

Appraised value - An opinion of value reached by an appraiser based upon knowledge, experience, and a study of pertinent data. Distinguished from FHA value.

Appraiser - Person who gathers information ahout a home that is necessary to rate the energy efficiency of the home.

Appreciation - Any increase in valuc. The opposite of depreciation.

Appurtenance - Anything-concrete or abstract - attached to the land and thus part of the property, such as a harn, garage, or easement.

Assessed valuation - The value that a taxing authority places upon real or perional property for calculating taxes owed. 
Audit - (1) Analysis of a specific building's consumption and potential to conserve utility-supplied energy; (2) an energy inspection typically associated with utility RCS (Residential Conservation Service) audits, which were mandated by Congress for larger utilities to provide until July 31 , 1990.

Avoided cost - The cost of supply avoided by an energy-efficiency program. Avoided costs include the cost of fuel and maintenance, and the costs of new capacity needs.

Azimuth - The angular distance between true south and the point on the horizon directly below the sun. Typically used as an input for opaque surfaces and windows in computer programs for calculating the energy performance of buildings.

Ballast - A device that provides starting voltage and limits the current during normal operation in electrical discharge lamps (such as fluorescent lamps).

Balloon mortgage - A mortgage with periodic installments of principal and interest that do not fully amortize the loan. The balance of the mortgage is due in a lump sum at a specific date in the future, usually at the end of the term.

Balloon payment - The unpaid principal amount of a mortgage or other long-term loan due on a specified date in the future, usually at the end of the term.

Bankrupt - A person, firm, or corporation who, through a court proceeding, is relieved from the payment of all debts after the surrender of all assets to a court-appointed trustee, or referee, for the protection of the creditors. In lieu of total relief from payment, the bankrupt may be required to make partial payments on a regular schedule, through the trustee or referee.

Baseline - The point from which energy savings are measured.

Basic qualifying ratio - The maximum generally acceptable qualifying ratio for a mortgage agency, e.g., for Fannie Mae and Freddic Mac, it is presently 28/36. This ratio is often adjusted upward or sometimes downward for compensating factors such as the borrower's credit worthiness.

Basis point - One one-hundredth of $1 \%$. For example, a one basis point profit on $\$ 1,000,000$ is $\$ 100$. Used to describe the amount of change in yield in many debi instruments, including mortgages. Do not confuse with Discount point.

Binder insurance - A written evidence of temporary hazard or title coverage that runs only for a limited time and must be replaced by a permanent policy. Neither HUD-FHA nor VA requires evidence of hazard insurance, but prudent lenders generally refuse to close loans without at least a binder.

Borrower - A mortgagor who receives funds in the form of a loan with the ohligation of repaying the loan in full with interest, if applicable.

British thermal unit (Btu) - A unit used to measure quantity of heat, defined as the quantity of energy necessary to raise the temperature of 1 lh of water $1^{\circ}$ Fahrenheit.

$\mathrm{Btu} / \mathrm{ft}^{2}$ - Energy consumption unit.

Building conde - The !ocal regulations that control design, construction, and materials used in construction

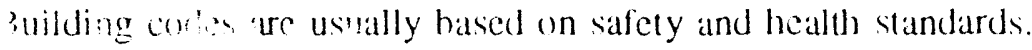


Building energy-efficiency standards - Building energy-efficiency standards establish the performance requirements for new residential and nonresidential buildings constructed or altered in California. (See California Code of Regulations, Title 24, Part 2, Chapter 2-53; regulating the energy efficiency of buildings constructed in California.)

Building envelope - The assembly of exterior partitions of a building that enclose conditioned spaces, through which thermal energy may be transferred to or from the exterior, unconditioned spaces, or the ground. (See California Code of Regulations, Title 24, Section 2-53()2.)

Building inspector - An employee of a local or state government building department whose responsibilities include reviewing huilding plans and/or inspecting building sites to determine whether or not they meet existing health, safety, and/or energy codes.

Buydown - Money advanced by an individual (builder, seller, etc.) to reduce the monthly payments for a home mortgage either during the entire term or for an initial period of years.

Cfm (cubic feet per minute) - A measure of flow rate.

Calculational systems - Systems usually based on one of the many building simulation models and an estimate of the amount of energy saved over some base case, which might he the current building code. The savings are translated into several forms: Btu/ $\mathrm{ft}^{2}$, points, which are equivalent to a certain amount of usage/savings, or other designations such as "stars."

California seasonal efficiency (CSE) - See Seasonal efficiency.

Cash equivalent value - A method of arriving at the appraised value of a property that takes sales and financing concessions into consideration when evaluating comparable properties. If, for example, a comparable property sold for $\$ 50,000$, but the seller paid a substantial discount to assist the buyer in arranging financing, it is assumed that the actual sale price was less. If the typical discount on a similar transaction would have been $\$ 2,000$, but the seller paid $\$ 4,000$, the "cash equivalent value" of the comparable property would be reduced to $\$ 48,000$. There is no standard in the appraisal industry for measuring cash equivalent value, but investors and mortgage insurers sometimes insist that cash equivalency be incorporated in appraised values.

Caulking - Material used to make an airtight seal by filling in cracks, such as those around windows and doors.

Certificate of deposit - A written document issued by a bank or other financial institution that is evidence of deposit with the issuer's promise to return the deposit plus earnings at a specified rate of interest. Some certificates of deposit include restrictions on redemption hefore maturity, which make them unacceptable as a source of funds for closing mortgage loans.

Certificate of eligibility - VA Form 26-8320) (Exhibit 7). Evidence that the veteran is eligible for VA loan guaranty henefits. The face of the form identifies the veteran. The reverse provides details on the status and extent of his ot her eligibility.

Certificate of reasonable value (CRV) - VA Form 26-1343, HUD Form 928(1)-5. A document issucd hy the VA establishing a maximum value and loan amount for a mortgage to be guaranteed by the VA. The CRV will also be accepted by HUD-FHA to estahlish the appraised value of the properiy for a motitgage to be iñisired by HUD-FHA. 
Certification programs - A program typically operated by utilities, home builders' organizations, or notfor-profit organizations representing interest parties. Energy-efficiency standards are developed using local area demographics, construction practices, and area climatic conditions. They usually include thermal envelope efficiency criteria and space conditioning efficiency crieria. Certification programs generally rely on a specified inspection/verification process to ensure rating consistency. Houses either pass or fail the inspection for energy efficiency.

Certified Mortgage Bankers (CMB) - The highest professional designation awarded to employees of member firms or individual members of the Mortgage Bankers Association of America.

Certified Review Appraiser (CRA) - The highest professional designation awarded to appraiser members of the National Association of Review Appraisers and Mortgage Underwriters.

Circuit - One complete run of a set of electric conductors from a power source to various electrical devices (appliances, lights, etc.) and back to the same power source.

Chiller - A device that cools water, usually to between $40^{\circ}$ and $50^{\circ}$ Fahrenheit, for eventual use in cooling air.

Clerestory - A wall with windows that is between two roofs at different levels. The windows are used to provide natural light for a building.

Climate - The meteorological conditions (including temperature, precipitation, humidity, and wind) that characteristically prevail in a particular region.

Climate zone (CZ) - One of 16 geographic zones in California defined by the California Energy Commission as having similar typical weather patterns. Climate zones are used to determine annual energy use in buildings in each climate zone.

Closing - The conclusion of a transaction. In real estate, closing includes the delivery of a deed, financial adjustments, the signing of notes, and the disbursement of funds necessary to the sale or loan transaction.

Closing costs - Money paid by any party to the transaction to effect the closing of a mortgage loan. Does not include prepaid expenses, apportionments, and the like, but does normally include an origination fee (almost always paid by the borrower), title insurance, survey, attorney's fees, ctc. In HUD-FHA transactions, all closing costs are added to the appraised value of the property to establish the FHA value on which the maximum insurable mortgage is hased. Closing costs paid by the borrower are added to the sales price to establish acquisition costs.

Coefficient of performance (COP) - Used to rate the performance of a heat pump, the COP is the ratio of the rate of useful heat output delivered by the complete heat pump unit (exclusive of supplementary heating) to the corresponding rate of energy input, in consistent units and under specific conditions. (See California Code of Regulations, Title 20, Section 16()2[c]|6].)

Combined hydronic space/water heating - A system in which both space heating and domestic water heating are provided by the same device or appliance.

Comfort conditioning - The process of treating air to simultaneously control its temperature, humidity, cleanliness, and distribution to meet the comfort requirements of the occupants of the conditioned space. 
Comfort zone - The range of temperatures over which the majority of persons feel comfortable (neither too hot nor too cold).

Commitment - An agreement, often in writing, between a lender and a borrower to loan money at a specified date in the future. The lender may or may not expect to fund the commitment.

Common area - Land or improvements on land that are designated for common use and enjoyment by all occupants, tenants, or owners.

Co-mortgager - A second borrower, not the spouse of the principal borrower, who assumes equal responsibility for the debt and a share in ownership of the property. Income and obligations of co-mortgagor are considered in the underwriting process as though he or she were the principal mortgagor. Contrast with Co-signor.

Comparables - An abbreviation for comparable properties used for comparative purposes in the appraisal process. Refers to facilities of reasonably the same size and location with similar amenities. Also properties that have been recently sold and have characteristics similar to the property under consideration, thereby indicating the approximate fair market value of the subject property.

Comparisons to housing stock data base - Systems that take actual utility bills and compare them with some average either based on "typical" bills for all customers or on subcategories based on building types.

Compensating factors - Any underwriting consideration that would justify the use o: higher debt-toincome qualifying ratios. Examples are large downpayment, excellent credit history, or a demonstrated ability to accumulate savings.

Component optimization system - Rating system performed by comparing homes to be rated to similar model homes that have already been appraised according to energy consumption and features.

Condenser - A heat exchanger in which the refrigerant, compressed to a hot gas, is condensed to liquid by rejecting heat.

Conditioned area (or space) - That portion of the building that is heated and/or cooled.

Conditioned floor area - The floor area of enclosed conditioned spaces on all floors measured from the interior surfaces of exterior partitions for nonresidential buildings and from the exterior surfaces of exterior partitions for residential huildings. (See California Code of Regulations, Title 24, Section 2-53(2).

Conditioned space - Enclosed space that is either directly conditioned space or indirectly conditioned space. (See California Code of Regulations, Title 24, Section 2-53(22.)

Conditioned space, directly - An enclosed space that is provided with heating equipment that has a capacity exceeding $10 \mathrm{Btu} /\left(\mathrm{hr}-\mathrm{ft}^{2}\right)$, or with cooling equipment that has a capacity exceeding 10) $\mathrm{Btu} /\left(\mathrm{hr}-\mathrm{ft}^{2}\right)$. An exception is if the heating and cooling equipment is designed and thermostatically controlled to maintain a process environment temperature less than $65^{\circ}$ Fahrenheit or greater than $85^{\circ}$ Fahrenheit for the whole space the equipment serves. (Sec California Code of Regulations, Title 24, Section 2-53(12.) 
Conditioned space, indirectly - Enclosed space that is not directly conditioned space with area weighted heat transfer coefficient ( $U$-valise) to directly conditioned spaces exceeding that to the outdoors or to unconditioned spaces; or through which air from directly conditioned spaces is transferred at a rate exceeding three air changes per hour. (See California Code of Regulations, Title 24, Section 2-5302.)

Condominium - A form of ownership of real property. The purchaser receives title to a particular unit and an undivided, or proportionate, interest in certain common areas. A condominium generally defines each unit as a separately owned space to the interior surfaces of the perimeter walls, floors, and ceilings. Title to the common areas is in terms of percentages and refers to the entire project less the separately owned units.

Condominium declaration - The basic condominium document that must be registered by the originating property owner before conveyance of the first unit sold. This declaration thoroughly describes the entire condominium entity, including each unit and all common areas, and specifies essential elements of ownership that permanently govern its operation. Also known as a master deed.

Conductance - The quantity of heat, in Btu, that will flow through $1 \mathrm{ft}^{2}$ of material in 1 hour, when there is a degree Fahrenheit temperature difference between both surfaces. Conductance values are given for a specific thickness of material, not per-inch thickness.

Conductivity (k) - The quantity of heat that will flow in 1 hour through $1 \mathrm{ft}^{2}$ of homogeneous material, 1 -inch thick, when there is a temperature difference of $1^{\circ}$ Fahrenheit bet'een its surfaces.

Convection - Heat transfer by the movernent of fluid.

Conventional energy-related practice - Any device, equipment, material, process, construction method, system, structure, or combination thereof as they relate to energy usage, that is common to a particular area and/or is required by local, state, or federal regulations or standards.

Conventional loan - A mortgage loan neither insured by HUD/FHA nor guaranteed by VA or the Farmers Home Administration.

Cooling capacity, latent - Available refrigerating capacity of an air conditioning unit for removing latent heat from the space to be conditioned.

Cooling capacity, sensible - Available refrigerating capacity of an air conditioning unit for removing sensible heat from the space to be conditioned.

Cooling capacity, total - Available refrigerating capacity of an air conditioning unit for removing sensible heat and latent heat from the space to be conditioned.

Cooling load - The rate at which heat must he extracted from a space in order to maintain the desired temperature within the space.

Cooling load temperature difference (CLTD) - A value used in cooling load calculations for the effective temperature difference, delta $T$, across a wall or roof that accounts for the effect of radiant heat as well as the temperature difference.

Cooling tower - A device for evaporatively cooling water hy contact with air. 
Correlation - The final step in the appraisal process in which the appraiser considers the three estimates of value derived from the cost, income, and market data approaches. The correlation process weighs the influence of each in relation to the type of property and the final estimate of value.

Co-signer - One who agrees to assume the debt obligation if the principal borrower defaults on mortgage payments. A co-signer assumes only personal liability and has no ownership interest in the property. His or her income and obligations are used in the underwriting process to reinforce the credit of the principal borrower. The co-signer's credit is not given equal weight with that of the principal borrower, but serves only as a compensating factor. Contrast with Co-mortgagor.

Cost approach to value - A means of valuation in which the value of a property is determined by computing the replacement value of improvements, depreciation, and the value of the land.

Cost effective - Producing the most economical outcome for consumers.

Credit rating - A rating to a prospective lender on the credit standing of a prospective borrower, used to help determine credit worthiness.

Credit report - A report to prospective lender on the credit standing of a prospective borrower, used to help determine credit worthiness.

Daylighting - The use of sunlight to supplement or replace electric lighting.

Daylighting control - A control system that varies the light output of an electric lighting system in response to variations in available daylight.

Debt - A sum of money due by certain and express agreement.

Debt/equity ratio - A ratio between the amount of capital borrowed and the amount of capital invested out-of-pocket or obtained through the sale of common stock; also known as the leverage ratio.

Debt-to-income ratio - see Qualifying ratio.

Debt-to-income ratio increase - see Ratio increase.

Deed - A document by which the ownership of land is transferred from one party to another.

Deed of trust - In some states, the document used in place of a mortgage. A type of security instrument conveying title in trust to a third party covering a particular piece of the property. It is used to secure the payment of a note. A conveyance of the title to land to a trustee as collateral security for the payment of a debt with the condition that the trustee will reconvey the title on payment of the debt, and with power of the trustee to sell the property and pay the debt in the event of a default on the part of the debtor.

Default - A breach or nonperformance of the terms of a note or the covenants of a mortgage.

Degree day - A unit, based upon temperature difference and time, used in estimating fuel consumption and specifying nominal annual heating load of a building. When the mean temperature is less than $65^{\circ}$ Fahrenheit, the heating degree days are equal to the total number of hours that the temperature is less than $65^{\circ}$ Fahrenheit for an entire year. 
Delta 'T - A difference in temperature. Often used in the context of the difference between the design indoor temperature and the outdoor temperature.

De minimis planned unit development - A type of common ownership. The owner not only owns the individual unit but has shared ownership interest in the common areas of the community. "De minimis" indicates a "minimal" amount of common area, usually less than $2 \%$ of total value. Large condominium developments with common areas, tennis courts, swimming pools, and other improvements do not qualify.

Demand-side management (DSM) - Utility programs designed to control energy consumption on the customer's side of the meter. Such programs include conservation/energy efficiency, load management, fuel substitution, and load building.

Density - The mass of a unit volume of a substance.

Depletable energy sources - (1) Electricity purchased from a public utility; (2) energy obtained from burning coal, oil, natural gas, or liquefied petroleum gases. (See California Code of Regulations, Title 24, Section 2-5302.)

Deposit - (1) A sum of money given to bind a sale of real estate, or (2) a sum of money given to assure payment or an advance of funds in the processing of a loan. Also known as Earnest money.

Deposit receipt - A form user to accept the earnest money that binds an offer to purchase real property. In many transactions, the deposit receipt is included in the sales contract.

Depreciation - A sum representing presumed loss in the value of a building or other real estate improvement resulting from age, physical wear, and economic or functional obsolescence.

Developer - A person or entity who prepares raw land for building sites and sometimes builds on the sites.

Diffuse radiation - Solar radiation, scattered by water vapor, dust, and other particles as it passes through the atmosphere, so that it appears to come from the entire sky. Diffuse radiation is higher on hazy or overcast days than on clear days.

Direct expansion (refrigeration) - Any system that, in operation between an environment in which heat is absorbed (heat source) and an environment into which unwanted heat is directed (heat sink) at two different temperatures, is able to absorb heat from the heat source at the lower temperature and reject heat to the heat sink at the higher temperature. The cooling effect is ohtained directly from a fluid called a refrigerant that absorbs heat at a low temperature and pressure, and transfers heat at a higher temperature and higher pressure.

Direct radiation - Radiation that has traveled a straight path from the sun, as opposed to diffuse radiation.

Direct solar gain - Solar energy collected from the sun (as heat) in a building through windows, walls, skylights, etc.

Directly conditioned space - See Conditional space, directly. 
Discount - In loan origination, a discount refers to an amount witheld from loan proceeds or collected siparately by a lender. In secondary market sales, a discount is the amount by which the sale price of a note is less than its face value. In both instances, the purpose is to adjust the yield upward, either in lieu of or in addition to interest. The rate or anount of discount depends on money markel conditions, the credit of the borrower, and the rate or terms of the note. Borrowers are prohibited from paying discounts associated with VA mortgages, although they may be paid by any other party. Interest rates and discounts are now freely negotiable with respect to HUDFHA transactions.

Discount point - Amount payable to the lending institution by the borrower or seller to increase the lender's effective yield. One point is equal to $1 \%$ of the loan.

Dry-bulb temperature - A measure of the sensible temperature of air.

Dual-duct system - A central plant heating, ventilating, and air conditioning (HVAC) system that produces conditioned air at two temperatures and humidity levels. The air is then supplied through two independent duct systems to the points of usage where mixing occurs.

Dual-paned (double-glazed) - Two panes of glass or other transparent material.

Duct - A passageway made of sheet metal or other suitable material used for conveying air or other gases at low pressures.

Earnest money - A sum of money given to (1) bind a sale of real estate, or (2) assure payment or an advance of funds in the processing of a loan; a deposit.

Easement - The right to the limited use of or enjoyment of land held by another. An easement is an interest in land-to enable sewer or other utility lines to be laid or to allow for access to a property.

Economic life - The period of time over which the energy-saving measure may reasonably he expected to perform the function for which it was designed or intended without major renewal or overhaul.

Economizer, air - A ducting arrangement and automatic control system that allows a heating, ventilating and air conditioning (HVAC) system to supply up to $100 \%$ outside air to satisfy cooling demands, even if additional mechanical cooling is required.

Economizer, water - A system that is capable of cooling the air supply by direct evaporation, evaporative cooling, or both, even if additional mechanical cooling is required.

Effective gross income (personal) - Normal annual income, including overtime, before deductions, that is regular or guaranteed. It may he from more than one source. Salary is generally the principal source, but other income may be significant and stable, and thus qualify. Effective gross income is used as the hasis for borrower qualification by most conventional lenders and private mortgage insurers.

Efficacy lighting - The ratio of light from a lamp to the electrical power consumed, including ballast losses, expressed as lumens per watt. (Sec California Code of Regulations, Title 24, Section 2-53(1).) 
Efficiency - The ratio of the useful energy delivered by a dynamic system (such as machine, engine, or motor) to the energy supplied to it over the same period or eycle of operation. The ratio is usually determined under specific test conditions.

Electricity - A property of the basic particles of matter. A form of energy having magnetic, radiant, and chemical effects. Electric current is created by a flow of charged particles (clectrons).

Electric resistance heater - A device that produces heat through electric resistance. For example, an electric current is run through wire coil with a relatively high electric resistance, thereby converting the electric energy to heat, which can be transferred to the space by fans.

Electric radiant heating - A heating system in which electric resistance is used to produce heat that radiates to nearby surfaces. There is no fan component to a radiant heating system.

Elevation - (1) The height above sea level (altitude); (2) a geometrical projection, such as a building on a plane perpendicular to the horizon.

Emissivity - The property of emitting radiation; possessed by all materials to a varying extent.

Emittance - The emissivity of a material, expressed as a fraction. Emittance values range from 0.05 for brightly polished metals to 0.96 for flat black print.

Endorsement - A writing on a negotiable instrument by which title to property mentioned therein is assigned and transferred. A notation added to an instrument after execution to change or clarify its contents. In insurance, coverage may be restricted or enlarged by endorsing a policy. In HUD-FHA loans, it is a notation formerly placed on the note and mortgage by HUD-FHA evidencing that the mortgage is insured under the National Housing Act. Although notes and mortgages are no longer actually "endorsed," the separate mortgage insurance certificate, when completed by HUD-FHA, serves the same purpose. See Mortgage insurance certificate.

Energy - The capacity for doing work. Forms of energy include thermal, mechanical, electrical, and chemical. Energy may be transformed from one form into another.

Energy budget - A requirement in the building energy-efficiency standards that a proposed building be designed to consume no more than a specified number of British thermal units (Btu), per year per square foot of conditioned floor area.

Energy-efficiency measures - Items that reduce a home's consumption of utility-supplied energy, including measures such as insulation, low-emissivity windows, and renewable energy technologies such as passive solar design and solar domestic hot water systems.

Energy-efficiency rating - A certification of a home's energy efficiency or a relative indication of its energy efficiency on a graduated scale.

Energy-efficiency ratio (EER) - The ratio of cooling capacity of an air conditioning unit in British thermal units per hour to the total electric input in watts under specified conditions. (Sec California Code of Regulations, Title 20, Section 16(02[c]|6].)

Energy-efficient mortgages (EEMs) - When a homeowner or home huyer applies for a home loan, at the time of purchase or refinance, he or she can roll the cost of needed energy improvements into the mortgage, amortizing the cost of the improvements over the life of the mortgage. 
Energy-efficient mortgage program - The energy improvement programs of the VA, FHA, Fannic Mac, Freddie Mac, and the Farmers Home Administration. These are national programs that are available to all home buyers and homeowners at the time of purchase or refinance.

Energy label - Label or sticker placed on the home's energy meter stating energy-efficiency rating of home.

Energy management system - A control system (often computerized) designed to regulate the energy consumption of a building by controlling the operation of energy consuming systems such as the heating, ventilating and air conditioning (HVAC), lighting, and water heating systems.

Energy rating - A designation of the relative efficiency of a property. In a larger sense, the rating would also include a prioritized energy improvement recommendation, estimates of energy and dollar savings, and documentation of efficiency and savings for the loan file if completed in conjunction with energy-efficient mortgages.

Energy cost savings - The difference between a home whose energy costs are being measured and a comparable home with no energy-saving construction or improvement features. For an existing energy-inefficient home, it is the difference between the home as it exists and the home after it has received energy-saving improvements.

Energy-saving construction or improvement features - Features that contribute to lowering of energy use in a residence. They include, but are not limited to the following: insulation, e.g., wall, ceiling, floor, slab, crawl, basement, window, door, etc.; air infiltration reduction, e.g., gaskets, caulking, weatherstripping, controlled mechanical ventilation, etc.; heating and cooling equipment, e.g., setback thermostats and high efficiency furnace, air conditioner, water heater, and fireplace; duct loss reduction; glazing, e.g., amount of glazing, R-value, solar fraction, solar orientation; and passive and active solar features.

Energy-saving measure - Any device, equipment, material, process, construction method, system, structure, or combination thereof that will result in a reduction of energy usage when compared with conventional energy-related practice in the area of the project.

Enthalpy - The quantity of heat necessary to raise the temperature of a substance from one point to a higher temperature. The quantity of heat includes both latent and sensible.

Equal Credit Opportunity Act (ECOA) - A federal law that requires lenders and other creditors to make credit equally available without discrimination based on race, color, religion, national origin, age, sex, marital status, or receipt of income from public assistance programs.

Equity - Net ownership; the difference between fair market value and current indebtedness, usually referred to as the owner's interest.

Escrow - (1) A transaction in which a third party, acting as the agent for the buyer and seller, carries out instructions of both and assumes the responsibilities of handling all the paperwork and disbursement of funds in a transfer of title and mortgage loan transaction. Escrows may also be established for any purpose, at any time, for example, in the administration of funds set aside to effect a "buydown" agreement among seller, buyer, and lender. (2) Funds included in the monthly mortgage payments to accumulate amounts necessary to pay property taxes, insurance premiums, etc., in the future are held in escrow, but the lender often acts as the escrow agent, especially if the lender is a depository institution. 
Escrow account - The segregated trust account in which escrow funds are held. The term is also used to describe the accounting of such funds.

Escrow agent - The person or organization having a fiduciary responsibility 60 both the buyer and the seller or other parties to see that the terms of the purchase/sale, loan, or other agreement are carricd out.

Escrow analysis - The periodic examination of escrow accounts to determine if current monthly deposits will provide sufficient funds to pay tax, insurance, and other bills.

Escrow arrangements - Procedures followed and agreements made in conjunction with establishing and scheduling disbursements from an escrow account.

Escrow payment - That portion of a mortgagor's monthly payment held by the lender or servicer to pay for taxes, hazard insurance, mortgage insurance, lease payments, and other items as they become due. Known as impounds or reserves in some states.

Evaporative cooling - Cooling by exchange of latent heat from water sprays, jets of water, or wetted material.

Exceptional method - An approved alternative calculation method that analyzes designs, materials, or devices that cannot be adequately modeled using public domain computer programs. Exceptional methods must be submitted to and approved by the California Energy Commission. (See California Code of Regulations, Title 20, Section 1409[b]3.) Two examples of exceptional methods are the controlled ventilation crawl space (CVC) credit and the combined hydronic space and water heating method.

Exfiltration - Air flow outward through a wall, building envelope, ctc.

Exhaust - Air removed deliberately from a space by a fan or other means, usually to remove contaminants from a location near their source.

External load controls - Fixed or movable shading elements (i.e., awnings, wing walls, overhangs, caves, shade screens, etc.) that control solar heat gain to exterior envelope components.

External shading device - Any object that diminishes the intensity of solar radiation before it strikes the exterior surface of a building.

Fan coil - A component of a heating, ventilating, and air conditioning (HVAC) system containing a fan and heating or cooling coil used to distribute heated or cooled air.

Fannie Mae -Term commonly used in referring to the Federal National Mortgage Association.

Farmers Home Administration (FmHA) - A government agency within the Department of Agriculture that operates under the Consolidated Farm and Rural Development Act of 1921 and Title V of the Housing Act of 1949. This agency provides financing to farmers and other qualified borrowers who are unable to obtain loans elsewhere.

Federal Home Loan Bank Board (FHLBB) - A regulatory and supervisory agency for federally chartered savings institutions. It oversees the operations of the Federal Savings and Loan Insurance Corporation and the Federal Home Loan Mortgage Corporation. 
Federal Home Loan Mortgage Corporation (FHL,MC) - A quasi-government agency that purchases conventional mortgages in the secondary mortgage market from insured depository institutions and HUD-approved mortgage hankers. It sells mortgage participation certilicates (PCs) secured by pools of conventional mortgage loans. Popularly known as Freddic Mac.

Federal Housing Administration (FHA) - A division of the Department of Housing and Urban Development. Its main activity is the insuring of residential mortgage loans made by private lenders. It sets standards for construction and underwriting. FHA neither lends money, plans, nor constructs housing.

Federal National Mortgage Association (FNMA) - A congressionally chartered corporation with private stockholders that purchases residential mortgages insured by FHA or guaranteed by VA, as well as conventional home mortgages. Popularly known as Fannie Mae.

Fee simple - Fee ownership is the greatest estate one can hold in land. Ownership rights under fee simple include the right to dispose of the property or pass it on to one's heir.

FHA value - The value established by FHA as the basis for determining the maximum mortgage that may be insured on a specific property. It includes the appraised value of the property and HUD-FHA estimate of closing costs.

First-time home buyer- A person who has not purchased a home in three years.

Fixed rate mortgage - A mortgage on which the interest rate is set for the term of the loan.

Fluorescent lamp - A tubular electric lamp coated on its inner surface with phosphor and containing mercury vapor. Bombardment by electrons from a cathode in the lamp provides ultraviolet light that causes the phosphor to emit visible light either of a selected color or closely approximating daylight.

Footcandle - A unit of illuminance on a surface that is 1 foot from a uniform point source of light of 1 candle and is equal to 1 lumen per square foot.

Forced air unit (FAU) - A central furnace equipped with a fan or blower that provides the primary means for circulation of air.

Framing effects - The effect of framing (wood or metal studs, joists, beams, etc.) on the overall U-value of a wall, roof, floor, window or other building surface. Framing generally increases the $U$-value and decreases the $R$-valtee of insulated surfaces.

Framing percentage - The area of actual framing in an envelope assembly. This percentage is used to calculate the overall $U$-value of an assembly.

Freddie Mac - Term commonly used in referring to the Federal Home Loan Mortgage Corporation.

Fuel blind - A rating system in which the energy fuel source is not specified.

Fuel neutral - Rating system that factors in heating and cooling equipment efficiency without favoring one energy type or technology.

Fuel source - Rating system that specifies a certain energy type of heating/cooling equipment. 
Functional obsolescence - A reduction in value caused by changes in taste, overcapacity, or inadequacy. Examples are outdated kitchen fixtures and outmoded room arrangement.

General lighting - Lighting designed to provide a substantially uniform level of illumination throughout an area, exclusive of any provision for special visual tasks or decorative effect. (See California Code of Regulations, Title 24, Section 2-5302.)

Gift letter - A letter certifying to the underwriter that funds in an applicant's account are truly a gift and need not be repaid.

Ginnie Mae - Term commonly used in referring to the Government National Mortgage Association.

Glazing - A covering of transparent or translucent material (typically glass or plastic) used for admitting light. Glazing retards heat losses from re-radiation and convection.

Government National Mortgage Association (GNMA) - A government corporation within the Department of Housing and Urban Development authorized to provide secondary mortgage financing primarily through the use of private capital. GNMA administers the mortgage-backed securities program that channels new sources of funds into residential financing through the sale of privately issued securities carrying a GNMA guaranty.

Graduated payment mortgage (GPM) - A type of flexible payment mortgage in which the payments increase for a specified period of time and level off.

Graduated rating programs - Rating programs that provide a relative indication of a home's energy efficiency on a variable scale selected by the sponsor.

Greenhouse effect - The characteristic tendency of some transparent materials (such as glass) to tran :mit radiation with relatively short wavclengths (such as heat). This tendency leads to heat buildup within the space enclosed by such a material.

Gross area - The area of a surface, including areas not helonging to that surface (such as windows and doors in a wall).

Gross rent multiplier - A figure used to compare rental properties. It gives the relationship between the gross rental income and sales price.

Hazard insurance - A contract wherehy an insurer, for a premium, undertakes to compensate the insured for loss on a specific property due to certain hazards.

Heat capacity - The amount of heat necessary to raise the temperature of a given mass ${ }^{\circ}$. Heat capacity may he calculated hy multiplying the mass hy the specific he'at.

Heat gain - An increase in the amnunt of heat contained in a space. resulting from direct solar radiation. heat flow through walls. windows, roof, and other huilding surfaces, and the heat given off hy people. lights, equipment. and other sources.

Heat loss - A decrease in the amount of heat contained in a space. resulting from heat flow through walls, windows. rouf. and other huildnes surfaces and from exfiltration of warm air. 
Heat pump - An air-conditioning unit capable of heating by refrigeration, transferring heat from one (often cooler) medium to another (often warmer) medium, and that may or niay not include a capability for cooling.

Heat transfer - Flow of heat energy induced by a temperature difference. Heat flow through a building envelope typically from a heated or hot area to a colled or cold area.

Heating load - The rate at which heat must be added to a space in order to maintain the desired temperature within the space.

Heating seasonal performence factor (HSPF) - A representation of the total heating output of a central air conditioning heilt pump in British thermal units during its normal usage period for heating divided by the to d electrical energy input in watt-hours during the same period, as determined using the test procedure specified in the California Code of Regulations, Title 20. Section 16()3(c)

Heating, ventilating, and air conditioning system (HVAC) - A system that provides heating, ventilating, and/or cooling within or associated with a building.

Highest and best use - The use of land and/or improvements that is most likely to produce the greatest net return over a given period of time.

Home energy rating system(s) (HERS) - HERS measure and rate on a scale the relative energy efficiency of any house, regardless of age, efficiency, or fuel use. The rating is based on the efficiency of the thermal envelope and the heating, ventilating, and air conditioning (HVAC) system and is obtained by on-site inspection and calculations. HERS calculations include estimates of annual energy performance and costs and recommendations for cost-effective energyefficiency improvements.

HERS characteristics - (1) Designed to rate, on a scale, the relative energy efficiency of any house-new and existing, efficient and inefficient; (2) provides a rating based on efficiency of the thermal envelope, space heating and cooling efficiency, and water heating efficiency; (3) estimates annual costs; (4) recommends improvement measures; (5) is fuel neutral; (6) requires on-site inspections and quality control; (7) typically, is state sponsored (or approved) and third-party delivered; (8) has goal of providing voluntary, market-driven incentives to encourage increased efficiency; and (9) provides documentation that a house meets or exceeds a minimum standard for efficiency designated at a point on the scale.

Home energy rater - The person trained and possibly certified to inspect a residence to collect all information needed to complete a home energy rating.

Home inspector - A person or company who provides home inspection services for a fee. Currently such rompanies limit their inspections to an assessment of the structural and/or health and safety features of a builcing.

Horsepower - Unit of power; work done at the rate of 745.7 watts. 
Housing and Urban Development, Department of (HUD) - The Departmient of Housing and Urban Development was established by the Housing and Urhan Development Act of 1965 to supersede the Housing and Home Finance Agency and give Cahinet status to the administration of the nation's housing and urban development programs. It is responsible for the implementation and administration of government housing and urban development programs. The hroad range of programs include community planning and development, low-rent public housing, mortgage insurance for residential mortgages (FHA), equal opportunity in housing, and research and technology.

Hydronic heating - A system that heats a space using hot water that may be circulated through a convection or fan coil system or through a radiant baseboard or floor system.

HUD-code manufactured homes - Homes built to the Manufactured Home Construction and Safety Standards (MHCSS); commonly known as mobile homes.

\section{Impound - See Escrow payment and Escrow account.}

Incandescent lamp - An electric lamp consisting essentially of a glass or quartz bulb evacuated or filled with an inert gas in wiich a filament, commonly of tungsten, gives off light when it is heated to incandescence by an electric current.

Income approach to value - The appraisal technique used to estimate real property value by capitalizing net income.

Indemnify - To protect against loss or damage; insure. To make compensation to for loss or damage.

Indirectly conditioned space - See Conditioned space, indirectly.

Indeer ais quality - Indoor environmental quality of a site.

Infiltration - The uncontrolled inward leakage of air through cracks and gaps in the building envelope, especially around windows and doors.

Infiltration barrier - A waterproof material placed on the outside or the inside of exterior wall framing to restrict inward air leakage while permitting the outward escape of water vapor from the wall cavity. (See California Code of R^gulations, Title 24, Section 2-5302.)

Insolation - The total amount of solar radiation (direct, diffuse, and reflected) striking a surface exposed to the sky.

Insulation, ther nal - A material having a relatively high resistance of heat flow and used principally to retard heat flow.

Insured loan - A loan insured by HUD-FHA or a private mortgage insurance company.

Internal shading device - Any object on the interior side of a window that reduces the intensity of solar radiation reaching the living or working areas. Internal devices are much less effective at preventing heat gain than exterior shading devices.

Investor - The holder of a mongage or the permanent lender for whom the mortgage hanker services the loan. Any person or institution that invests in mortgages. 
Judgment - Final determination by a court of the rights and claims of the parties to an action.

KBtu - 1000 Btu.

Kilowatts (kW) - 1000 watt.

Kilowatt-hour (kWh) - 1000 watt-hour.

Latent cooling capacity - See Cooling capacity, latent.

Latent heat - A change in the heat content that occurs without a corresponding change in temperature, usually accompanied by a change of state (as from liquid to vapor during evaporation).

Latent load - The cooling load caused by moisture in the air.

Latitude - The angular distance north or south of the equator, measured in degrees of arc.

Leasehold - An estate or interest in an estate in real property held by virtue of a lease. A leasehold estate has a specific duration. Some states allow leases up to 99 years and consider a property without oral testimony.

Lien - A legal hold or claim of one person on the property of another as security for a debt or charge. The right given by law to satisfy debt.

Life-cycle cost - Amount of money necessary to own, operate, and maintain a building over its useful life.

Lighting power density - Total lighting power consumption fer unit area, typically measured in watts per square foot.

Liquidity - The ability of an individual or business to quickly convert assets into cash without incurring a considerable cost.

Load management programs - Programs that have the effect of reducing electric peak demands or shifting electric demand from the hours of peak demand to nonpeak time periods.

Loan-to-value ratio (LTV) - (1) The relationship between the amount of the mortgage loan and the appraised value of the security, expressed as a percentage of the appraised value. (2) In HUDFHA transactions, the relationship between the amount of the mortgage loan and "FHA value," which is the sum of the appraised value of the property and the estimated closing costs. See also FHA value.

Low-e - A special coating that reduces the emissivity of a window assembly, thereby reducing the heat transfer through the assembly.

Lumen - A measure of the amount of light available from a light source equivalent to the light emitted hy one candle.

Lumen maintenance control - An electrical control device designed to vary the electrical consumption of a lighting system in order to maintain a specified illumination level. 
Lumens/watt - A measure of the efficacy of a light fixture; the number of limens output per watt of power consumed.

Luminaire - A complete lighting system consisting of a lamp or lamps together with the part designed to distribute light, to position and protect the lamps and to connect the lamps to the power supply. (See California Code of Regulations, Title 20, Section 2-1602[i].)

Lux - A unit of illumination equal to the direct illumination on a surtace that is everywhere 1 meter from a uniform point source of 1 candle; a unit of illumination that is equal to 1 lumen per square meter. See also Footcandle.

Manufactured house - See Modular house and Mobile home.

Market approach to value - In appraising, the market value estimate is predicted upon actual prices paid in market transactions. It is a process of correlation and analysis of similar recently sold properties.

Market value - The most probable price that a property should bring in a competitive and open market under all conditions requisite to a fair sale, the buyer and seller each acting prudently and knowledgeably, and assuming the price is not affected by lender stimulus.

Mechanical systems - See Heating, ventilating, and air cenditioning (HVAC) system.

Member, Appraisal Institute (MAI) - The highest professional designation awarded by the American Institute of Real Estate Appraisers.

Mobile home - A factory-assembled residence consisting of one or more modules in which a chassis and wheels are an integral part of the structure and that can be made ready for occupancy without removing the chassis and/or wheels. See also Modular house.

Model conservation standards - Energy-efficiency standards created by the Northwest Power Planning Council.

Modular house - A factory-assembled residence built in units or sections, transported to a permanent site, and erected on a foundation. The term excludes mobile homes. See also Mobile home.

Mortgage - A conveyance of an interest in real property given as a security for the payment of a debt. In its simplest form, the mortgage permits foreclosure if the deht is not paid, but the foreclosure is usually a judicial proceeding in couri. After foreclosure, the property is then sold, usually by an officer of the court, to satisfy the deht.

Mortgage discount - The difference hetween the principal amount of a mortgage and the amount it actually sells for. Sometimes called points, loan brokerage fee, or new loan fec. The discount is computed on the amount of the loan, not the sales price.

Mortgagee - A person or firm to whom property is conveyed as security for a loan made hy such a firm; a creditor. 
Mortgage insurance certificate (MIC) - The certificate issued by HUD/FHA as evidence that the mortgage has been insured. This certificate is evidence that a contract of mortgage insurance exists between HUD/FHA and the lender, incorporating the HUD/FHA regulation identified in the certificate.

Mortgage insurance premium (MIP) - The consideration paid by a mortgage for mortgage insurance either to HUD-FHA or to a private mortgage insurance (PMI) company. Although the premium is paid to the insurer by the lender, it is usually collected, in turn, from the horrower.

Mortgager - The borrower or owner in a mortgage transaction who pledges property as a security for a debt.

Nationaillocal threshold - That point on the uniform scale at which energy-efficient mortgage benefits kick in. This would be a national threshold if it is set nationally, a local threshold if it is set locally.

Net worth - The value of all assets, including cash, less total liabilities. It is often used as an underwriting guideline to indicate credit worthiness and financial strength.

Neutral third party - An individual or group that does not have any financial interest in the mortgage transaction.

Nondepletable energy source - Energy that is not obtained from depletable energy sources. (See California Code of Regulations, Title 24, Section 2-5302.)

Numerical rating - Energy-efficiency rating system based on a numerical scale. Numbers vary, but an example would be a scale of $1-100$, where 100 is the best rating.

Obsolescence - The loss of value due to reduced usefulness resulting from outmoded physical features, hy becoming less suitable for use or by other economic influences.

Occupancy sensor - A control device that senses the presence of a person in a given space, zommonly used to control lighting systems in buildings.

Origination fee - A fee or charge for the work involved in the evaluation, preparation, and submission of a proposed mortgage loan. Often improperly considered part of the discount.

Originator - A person who solicits builders, hrokers, and others to obtain applications for mortgage loans. Origination is the process by which the mortgage banker brings into being a mortgage secured by real property.

Outside air - Air taken from outdoors and not previously circulated through the heating, ventilating, and air conditioning (HVAC) system.

Orientation - The position of a building relative to the points of a compass.

Overhang - Any horizontal projection that serves as a shading element for a window.

Par - The principal amount of a mortgage with no premium or discount. 
Partial load - An electrical demand that uses only part of the electrical power available. (Sec California Code of Regulations, Title 24, Section 2-5342|e|2.)

Pass/fail rating - A rating system in which an appraised home will "pass" if it meets or exceeds a predetermined level of energy efficiency.

Passive solar gain - Solar energy that enters the building, providing heating and/or daylighting to the building.

Passive solar system - A solar heating or cooling system that uses no external mechanical power to move the collected solar heat.

Passive solar technologies - Technologies that combine architecture to benefit from solar radiation incidence on buildings for heating, cooling, and lighting, with good conservation techniques for the building envelope and energy-efficient equipment and controls. Passive solar technologies are typically sunspaces, direct gain systems, and thermal storage wall systems.

Peak load - The highest electrical demand within a particular period of time.

Percolation test - A test given to soil to determine its water seepage capacity when the use of a septic tank is being considered. Also called "perc test."

Performance-based rating system - System that utilizes energy consumption data derived from past household utility bills to calculate an estimate of the property's total annual energy use.

Performance standard - An energy-efficiency standard that requires a building to meet a specified level of energy consumption, but does not require specific energy-efficiency features.

Perm - The measurement of water vapor through different materials measured on perm-inch (mass of water vapor moving through a unit area in unit time).

Photocell - A device that produces an electric reaction to visible radiant energy (light).

Physical approach to value - An appraisal method whereby pre serty value is derived hy estimating the replacement cost of improvements, less estimated depreciation, plus estimated land value by use of market data. Synonymous with the cost of approach.

Physical depreciation - The loss of value by real property resulting from wear and tear, disintegration, or action of the elements that can be either curable or incurable.

Pipeline - Term used to express loan applications in process up until closing or until the mortgage is sold. Used when analyzing mortgage loan inventory and commitment coverage.

PITI - Principal, interest, taxes, and insurance.

PITI + E - A borrower qualification method that incorporates energy efficiency in the deht-to-income ratios by adding the energy operating cost for the candidate house to other elements of the housing expense. 
PITI - ES - A borrower qualification method that incorporates energy efficiency in the debt-10-income ratios by deducting the energy savings of the candidate house from other elements of the housing expense.

PITI + ES - A borrower qualification method that incorporates energy efficiency in the debt-to-income ratios by adding energy savings to the allowed portion of gross income. Energy savings is added to the income portion of the deht-to-income ratio after gross income has heen multiplied by the underwriting guideline allowed housing expense ratio.

PITI ratio - The principal, interest, taxes, and insurance payment-to-income ratio used in mortgagelending decisions.

Planned unit development (PUD) - (1) A comprehensive development plan for a large land area. It usually includes residences, roads, schools, recreational facilities, and service areas, plus commercial, office, and industrial areas. (2) A subdivision having lots of areas owned in common and reserved for the use of some or all of the owners of the separately owned lots.

Plat (plot) - A map representing a piece of land subdivided into lots with streets, boundaries, easements, and dimensions shown thereon. It is usually recorded and made part of the public record.

Plot plan - A layout of improvements on a site, including their location, dimensions, and landscapes. It is generally part of the architectural plan.

Point - An amount equal to $1 \%$ of the principal amount of an investment or note. Loan discount points are a one-time charge assessed at closing by the lender to increase the yield on the mortgage loan to a competitive position with other types of investments.

Positive cash flow - A situation in which the energy cost savings from an energy-efficiency improvement exceeds the payment for the improvement in a given time period.

Premium - The amount, often stated as a percentage, paid in addition to the face value of a note or bond. The opposite of discount. Also, the charge for insurance coverage.

Prescriptive standard - An energy-efficiency standard that specifies the energy-efficiency features that must be included in a building.

Prescriptive systems - Systems that assign points to various energy design features, such as insulation, weather-stripping, etc., and rate the house according to the total number of accumulated points.

Principal - The amount of deht, exclusive of accrued interest remaining on a loan. Before any principal has heen repaid, the total loaned amount is the principal.

Private mortgage insurance - Insurance written hy a private company protecting the mortgage lender against loss occasioned by a mortgage default.

Processing - The preparation of a mortgage loan application and supporting documents for consideration hy a lender or insurer.

Programmable controller - A device that controls the operation of electrical equipment (such as air conditioning tunits and lights) according to a preset time and schedule. 
Qualifying ratio - Percentage ratios that compare the borrowers' anticipated monthly fixed housing expense and total monthly obligations to the borrowers' stable monthly gross income for the purpose of evaluating the likelihood of meeting expenses involved in homeownership.

Quality assurance - A system of controls and actions that ensure and verify conformity (within agreed upon tolerance) to a set of values, norms, standards, or expectations established by a recognized authority.

Radiant barrier - A device designed to reduce or stop the flow of radiant energy.

Radiant energy - Energy transferred by the exchange of electromagnetic waves from a hot or warm object to one that is cold or cooler. Direct contact with the object is not necessary for the heat transfer to occur.

Rater - A person trained and certified to complete energy ratings of buildings under an approved rating system.

Rating - A designation of a specific home on a rating scale designed to communicate its level of energy efficiency.

Rating tool - A certified procedure for calculating total annual energy consumption and costs of a home and for assigning a rating that establishes how the efficiency of a given home compares to the efficiency of all other homes.

Ratio increase - The process of increasing the standard debt-to-income ratio in the mortgage transaction by an incremental addition based on favorable financial attributes of a particular borrower.

Real Estate Settlement Procedure Act (RESPA) - A federal law that requires lenders to provide home mortgage borrowers in advance with information of known or estimated settlement costs. RESPA also limits the amount lenders may require to be held in escrow for real estate taxes and insurance, requires the disclosure of known settlement costs to both buyers and sellers by the person conducting the settlement, and outlaws certain referral fees.

Recool - The sensible cooling of air that has heen previously heated by heating, ventilating, and air conditioning (HVAC) systems serving the same huilding. (See California Code of Regulations, Title 24, Section 2-53(2.)

Recovered energy - Energy utilized from an energy system that would otherwise he wasted. (See California Code of Regulations, Title 24, Section 2-53()2.)

Recovery efficiency (thermal efficiency) - In a water heater, a measure of the percentage of heat from the combustion of gas that is transferred to the water as measured under the specitied test conditions. (See California Code of Regulations, Title 2(), Section 16(12[1]|7].)

Refinancing - The repayment of a deht from the proceeds of a new loan usiag the salme property as security.

Reflectance - The ratio (expressed as a percentage) of the amount of light reflected by a surface perpendicular to the glass. The light that is not reflected is either absorhed by the material or transmitted through it. 
Refrigerant - A fluid such as freon that is used in cooling devices to absorb heat from surrounding air or liquids as it evaporates.

Registered Mortgage Underwriter (RMU) - A professional designation awarded by the National Association of Review Appraisers and Mortgage underwriters in recognition of significant skills and experience in underwriting mortgage loans.

Reheat - The heating of air that has been previously cooled either by mechanical refrigeration or economizer cooling systems.

Remodeling - Additions, modernization.

Renewable energy technologies - The use of, as resources, the energy inherent in sunlight and the direct and indirect results of its impact on our planet (photons, wind, falling water, temperature differentials, and plant matter), gravitational forces (the tides), and the Earth's heat. These technologies at the sites of homes tend to be limited to: passive solar space heating, cooling, and lighting; solar water heating; active solar space heating; photovoltaic generation of electricity; biofuel appliances; and wind generation of electricity.

Renovation - Major rebuilding of existing building.

Representative structure - A building or facility similar in all ways to the proposed or existing building or facility with the exception that conventional energy-related practice has been substituted for the proposed energy-saving measures.

Reproduction cost - The money required to reproduce the building using the same or equivalent materials, design, and construction methods, less an allowance for depreciation; an clement of the cost approach method of appraisal.

Residential buildings energy conservation - To institutionalize energy costs and savings into the decision process for home buying, selling, appraising, lending, and remodeling.

Resistance, thermal - The reciprocal of thermal conductance. See also R-value.

Resource value - Extent to which energy efficiency and load management programs reliably reduce utilities' fuel and/or capacity needs.

Retrofit - A modification to an existing building. An energy retrofit is a retrofit that affects the energy performance of the building.

Return on investment (ROI) - Value of an energy-saving measure during its economic life.

Risk - Two kinds of risk are recognized by the mortgage lending community: (1) underwriting risk-the degree of probability of default by a borrower; and (2) default risk-the amount or quantity $i f$ loss due to default or nonpayment.

R-value - A unit of thermal resistance used for comparing insulating values of different material. The higher the $R$-value of a material, the greater its insulating properties and the slower the heat flows through it. 
Sales contract - A deliherate written agreement hetween competent parties stating terms and conditions of sale.

Scaled HERS - A system that uses a scale of energy efficiency designed to rank any given home against other homes in the area. Nonscaled HERS, often called certification or prescriptive prograns, use one or more benchmarks of energy efficiency instead of a continuous scale. Some HERS combine both-using a scale to rate the home and some designation or lahel linked to a few specific thresholds on the scale.

Scoring system - A rating system in which points can be obtained for particular levels of various energyefficiency features.

Seasonal efficiency (SE) - A measure of the percentage of heat from the combustion of gas and from associated electrical equipment transferred to the space being heated during a year under specified conditioned. (See California Code of Regulations, Title 20, Section 1602[e][11].)

Seasonal energy-efficiency ratio (SEER) - The total cooling output of a central air conditioning unit in British thermal units during its normal usage period for cooling divided by the total electrical energy input in watt-hours during the same period, as determined using specified federal test procedures. (See California Code of Regulations, Title 20, Section 1602[c][11].)

Seasoned loans - Mortgage loans that are of a certain age. Some investors require that loans be 1 or 2 years old prior to purchase.

Secondary mortgage market - A system whereby lenders and investors buy existing mortgage or mortgage-backed securities and in doing so provide greater availability of funds for additional mortgage lending by banks, mortgage bankers, and savings and loan associations.

Sensible cooling: capacity - See Cooling capacity, sensible.

Sensible heat - Heat that results in a temperature change.

Setback thermostat - See Thermostat, setback.

Shade screen - A screen affixed to the exterior of a window or other glazed opening designed to reduce the solar radiation reaching the glazing.

Shading - (1) The protection from heat gains due to direct solar radiation: (2) Shading is provided hy (a) permanently attached exterior devices, glazing materials, adherent materials applied 1. the glazing, or an adjacent huilding for nonresidential huildings, hotels, motels and high-rise apartments, and hy (h) devices affixed to the structure for residential huildings. (See Califirnici Code of Regulations, Title 24, Section 2-53(12.)

Shading coefficient - The ratio of solar heat gain through a specilic glazing system to the total solar heat gain through a single layer of clear, double-strength glass.

Shell area - The area of those elements of the huilding that form the intertace hetween the outside and inside of the building.

Shipping - Preparing and sending the complete package of mortgage documents to the investor. 
Side fins - Vertical shading elements mounted on either side of a glazed opening that blocks direct solar radiation from the lower, lateral portions of the sun's path.

Site-built home - Homes huilt on-site, including modular, sectional, panelized, and precut homes.

Site energy - The energy consumed at a building location or other end-use site.

Site-specific rating system - Systems that are actually computer programs based upon a computer simulation that represents heat transfer in a specific residential structure within a defined climatic region.

Skylight - Any opening in the roof surface that is glazed with a transparent or translucent material. (See California Code of Regulations, Title 24, Section 2-5302.)

Sky temperature - The equivalent temperature of the clouds, water vapor, and other atmospheric elements that make up the sky in which a surface can radiate heat.

Society of Real Estate A ppraisers (SREA) - The society awards the following professional designations: Senior Residential Appraiser (SRA); Senior Real Property Appraiser (SRPA); and Senior Real Estate Analyst (SREA).

Solar collector - A component of an active or passive solar system that absorbs solar radiation to heat a transfer medium which, in turn, supplies heat energy to the space or water heating system.

Solar heat gain - Heat added to a space duc to transmitted and absorbed solar energy.

Solar heat gain factor - An estimate used in calculating cooling loads of the heat gain due to transmitted and absorbed solar energy through $1 / 8$-inch-thick clear glass at a specific latitude, time, and orientation.

Solar radiation - Electromagnetic radiation emitted by the sun.

Source energy - All the energy used in delivering energy to a site, including power generation, Iransmission, and distribution losses, 10 perform a specific function such as space conditioning, lighting, or water heating. Approximating 3 watts (or $10.239 \mathrm{Bth}$ ) of energy is consumed to deliver I wall of usable electricity.

Specific heat - In heal units, the quantity of heat, in British thermal units, needed to raise the temperature of 1 pound of material $1^{\circ}$ Fathrenheit.

Stakeholders - Groups or individuals essential to the formation and implementation of a program and directly alfected hy its outcome.

Standby loss - A measure of the losies from a water heater tank. When expresicd als a percentage.

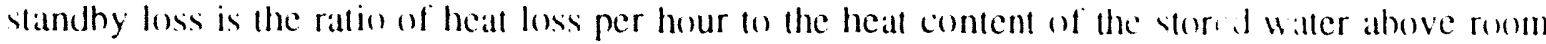
temperature. When exi ressed in watts, standhy loss is the heal lost per houm, per square foot of lank surface area. (See California Code of Regulations, Title 20, Section 16(12|e||13|.)

star rating approach - Ratting system in which stars are given to reflect energy efliciency of a home. For example, a rating of four stars on a scale of one to four stars leptresems the hes rating possible. 
Steady-state efficiency - A performance rating for the space heaters; a measure of the percentage of heal from combustion of gas that is transferred to the space heing healed under specified steady-state corditions. (See California Code of Regulations, Title 20, Section 161)2|f||6|.)

Storage-type water heater - A water heater that heats and stores water at a thermostatically controlled temperature for delivery on demand. (See California Code of Regulations, Title 20, Section $16(2|f||6|$.

Stretch mortgages - Enhanced qualification ratios.

Study period - The period of time equal to economic life of the energy-saving measure or 25 years, whichever is shorter.

Submetering - Breaking down the utility metering of a building to determine the proportionate energy use of specific huilding systems and appliances.

Survey - A measurement of land, prepared by a registered land surveyor, showing the location of the land with reference to knriwn points, its dimensions, and the location and dimensions of any improvement.

Surveyor's certificate - A formal statement signed, certified, and dated by a surveyor giving the pertinent facts about a particular property and any easements or encroachments affecting it.

Sweat equity - Equity created in a property by the performance of work or labor by the purchaser or borrower. It directly increases the value of the property.

System - A combination of equipment and/or controls, accessories, interconnecting means, and terminal elements by which energy is transformed to perform a specific function, such as climate control, service water heating, or lighting. (See California Code of Regulations, Title 24, Section 2-53(2.)

System program - A program that provides a certification for homes meeting an energy-efficiency standard or a rating of a home's relative energy efficiency.

Task lighting - Lighting designed specifically to illuminate one or more task locations, and generally confined to those locations. (See California Code of Regulations, Title 24, Section 2-53(22.)

Temperature - Degree of hotness or coldness measured on one of several arhitrary scales hased on some ohservable phenomenon (such as the expansion of mercury).

Term - The period of time hetween the commencement date and the termination date of a note, mortgage, legal document, or other contract.

Therm - $1(0),()()$ British thermal units (Btu).

Thermal break (thermal barrier) - An element of low heat conductivity placed in such a way as to reduce or prevent the flow of heat. Some metal framed windows are designed with the thermal hreaks to improve their overall thermal performance. 
Thermal energy storage - A technology that lowers the amount of electricity needed for comfort conditioning during utility peak load periods. A building's thermal energy storage system might, for example, use off-peak power to make ice or chill water at night, later using the ice or chilled water in a power saving process for cooling during the day. See also Thermal mass.

Thermal envelope - The building's shell-walls, foundation, floors, ceiling, windows, doors, and roof.

Thermal mass - A material used to store heat, thereby slowing the temperature variation within a space. Typical thermal mass materials include concrete, masonry, brick, tile and mortar, water and rock, or other materials with high heat capacity.

Thermostat - An automatic control device designed to be responsive to temperature and typically used to maintain set temperatures by cycling the heating, ventilating, and air conditioning (HVAC) system.

Thermostat, setback - A device containing a clock mechanism, which can automatically change the inside temperature maintained by the heating, ventilating, and air conditioning (HVAC) system according to a preset schedule. The heating or cooling requirements can be reduced when a building is unoccupied or when occupants are asleep. (See California Code of Regulations, Title 24, Section 2-5352[h].)

Title - The evidence of the right to or ownership in property. In the case of real estate, the documentary evidence of ownership is the title deed that specifies in whom the legal estate is vested and the history of ownership and transfers. Title may be acquired through purchaser, mortgagee, or otherwise.

Title insurance policy - A contract by which the insurer, usually a title insurance company, agrees to pay the insured a specific amount for any loss caused by defects in title to real estate, wherein the insured has an interest as purchaser, mortgagee, or otherwise.

Title search - An examination of public records, laws, and court decisions to disclose the past and current facts regarding ownership of a real estate.

Ton of cooling - A useful cooling effect equal to $12,000 \mathrm{Btu}$ hours.

Townhouse - A residential unit on a small lot that has coincidental exterior limits with other similar units. Title to the unit and its lot is vested in the individual buyer with a fractional interest in common areas, if any. Sometimes called a "row house."

Transmission - Heat transferred per unit of time; in thermodynamics, a general term for heat travel.

Transmittance - The time rate of heat flow per unit area under steady conditions from the air for other fluid) on the warm side of a barrier to the air (or fluid) on the cool side, per unit temperature difference between the two sides.

UA - A measure of the amount of heat that would be transferred through a given surface or enclosure (such as a building envelope) with a $1^{\circ}$ Fahrenheit temperature difference between the two sides. The UA is calculated by multiplying the $U$-value by the area of the surface (or surfaces). 
Unconditioned space - A space that is neither directly nor indirectly conditioned space, which can he isolated from conditioned space by partitions and/or closeable doors. (See California Code of Regulation, Title 24, Section 2-5302.)

Underwriting - The analysis of risk and the matching of it to an appropriate rate and term. Underwriting involves an analysis of the property, as revealed in the appraisal report, as acceptable and adequate security for the loan and an analysis of the borrower's ability and probable willingness to repay the loan. Risk may also be affected by other factors, such as loan-to-value ratios, the presence or absence of mortgage insurance, etc.

Uniform - Common, standardized, with no variations in material characteristics.

Uniform Commercial Code (UCC) - A comprehensive law regulating commercial transactions. It has been adopted, with modifications, by most states.

Utility audit - A formal review of a house's energy use conducted by a utility company representative, with recommendations for energy-efficiency measures, such as weatherstripping, caulking, and insulation.

U-value (coefficient of heat transmission) - The rate of heat loss, in British thermal units per hour, through a square foot of a surface (wall, roof, door, or other building surface) when the difference between the air temperature on either side is $1^{\circ}$ Fahrenheit. The $U$-value is the reciprocal of the $R$-value.

Valuation - Estimation of value or price though appraisal.

Value-in-use - The value of an economic good to its owner/user/inventor based upon its expected productivity or savings.

Vapor barrier - A material with a permanence of one perm or less which provides resistance to the transmission of water vapor. (See California Code of Regulations, Title 24, Section 2-5302.)

Variable air volume system (VAVS) - A mechanical heating, ventilating, and air conditioning (HVAC) system capable of serving multiple zones, which controls the temperature maintained in a zone. by controlling the amount of heated or cooled air supplied to the zone.

Ventilation - The process of supplying or removing air by natural or mechanical means to or from any space. Such air may or may not have been conditioned or treated.

Verification of employment (VOE) - A form that requests and secures verifications of the applicant's current or former employment. VA Form 26-8497, HUD Form 6233/92004-g, and Fannie Mae Form 1005 are typically used for verification of employment.

Veterans Administration (VA) - An independent agency of the federal government cre ited in 1930. The Servicemen's Readjustment Act of 1944 authorized the agency to administer a variety of henelit programs designed to facilitate the adjustment of returning veterans to civilian life. The VA home loan guaranty program is designed to encourage lenders to offer long-term, low-downpayment mortgages to eligible veterans by guarantecing the lender against loss. VA losses, if any, are paid with the appropriated funds. 
Visible light transmittance - The ratio of visible light transmitted through a substance to the total visible light incident on its surface.

Water heater - An appliance for supplying hot water for purposes other than space heating or pool heating. (See California Code of Regulations, Title, 20, Section 16(22[f]|8].)

Water table - Distance from the ground surface to a depth at which natural groundwater is found.

Watt - A unit of measure of electric power at a point in time, as capacity or demand.

Watt-hour - One walt of power expended for 1 hour.

Weatherization - Retrofitting a house's envelope with basic energy-efficiency measures, such as weatherstripping, caulking, and insulation.

Weatherstripping - Specially designed strips, seals, and gaskets installed around doors and windows to limit air leakage.

Wet-bulb temperature - The temperature at which water, hy evaporating into air, can bring the air to saturation at the same temperature. Wet-bulb temperature is measured by a wet-hulb psychrometer.

Whole-house fan - A system capable of cooling a house by exhausting a large volume of warm air when outside air is cool.

Wraparound mortgage - A refinancing technique involving the creation of a subordinate mortgage that includes the balance due on the existing mortgage(s) plus the amount of the secondary or junior lien.

Yield - In real estate, the term refers to the effective annual amount of income heing accrued on an investment; expressed as a percentage of the price originally paid.

Zonal control - A method of designing and controlling the heating, ventilating, and air conditioming (HVAC) system of a residence so that living areas can be maintained at a different temperature than sleeping areas, using independent sethack thermostats. If specilic requirements are met, zonal control can earn a credit toward compliance with building energy efficiency standards.

Zone - (1) In the context of a heating, ventilating, and air conditioning (HVAC) system: a space or group of spaces served hy an HVAC system or portion of an HVAC system controlled by a single thermostat or other control device; (2) A space or group of spaces within a huilding with sufficiently similar comfort conditioning requirements so that comfort conditions can he maintained throughout by a single control device. (See California Code of Regulations, Title 24, Section 2-53(2).

Zoning - The act of city or county authorities specifying the type of use to which a preperty may be aut in specific areas. 


\section{Bibliography}

Alaska Craftsman Home. n.d. Inside View of an Alaskan Craftsman Home. (Brochure).

Alliance to Save Energy. n.d. Home Energy Rating Systems: Proposal for a Uniform National Program. Washington, DC: Alliance to Save Energy.

1987. "An Evaluation of the Connsave Energy-Efficient Mortgage Program." Washington,

DC: Alliance to Save Energy. October.

1988. "Making Housing More Affordable Through Energy Efficiency." Washington, DC: Alliance to Save Energy.

1989. "Energy Efficient Mortgages: Proposal for a Uniform National Program." Washington,

DC: Alliance to Save Energy.

1990. "Home Energy Ratings Urged Nationwide: EEMs Should Be Updated, Streamlined." Energy Conservation Digest, 13, 61-65.

Alliance to Save Energy, American Council for an Energy Efficient Economy, Natural Resources Defense Council, and Union of Concerned Scientists. 1991. America's Energy Choices: Investing in a Strong Economy and a Clean Environment. Cambridge, MA: Union of Concerned Scientists.

American Gas Association. 1989. Affordable Housing Marketing Strategies for Implementing Energy Efficient Mortgages. Arlington, VA: American Gas Association.

American Society of Heating, Refrigerating, and Air-Conditioning Engineers Technical Evaluation Committee. May 1989. "Recommendations for Energy Conservation Standards for New Residential Buildings." Volume 3: Introduction and Background to the Standard Development Effort. Richland, WA: Pacific Northwest Laboratory.

Andrews, S. February 1991. "Lender Input to Energy Efficient Mortgage Process, During Your February 19th Monthly Meeting." Memorandum to Members of Colorado Mortgage Bankers Association. Denver, CO: City and County of Denver, Department of Health and Hospitals.

Architectural Energy Corporation. Fehruary 8, 1990. "Simplified Building Inputs Feasihility Study." Boulder, CO: Colorado Office of Energy Conservation.

The Arizona Energy Office of the Department of Commerce. 1991. "Home Energy Rating System Tested." Arizona Energy News, Spring:1,8.

Balcomb, J. D., R. W. Jones, R. McFarland, and W. O. Wray. 1984. Passive Solar Heating, A I)esign Manual. Atlanta, GA: American Society of Heating, Refrigeration and Air-Conditioning Engineers.

Barnet, C. and P. Thor. June 1990). Construction Cost Analysis, Residential Comstruction Demomstration Project, Cycle II. Portland, OR: Bonneville Power Administration.

Baylon, D., B. Davis, I. Brown, M. Kennedy, M. Luhliner, and S. Onisko. March 7, 199)1. "Manufactured Homes Thermal Analysis and Cost Effectiveness Report." Ecotople. Portland, OR: Bonneville Power Administration. 
Berryman, J. 1989. GMAC: Energy Efficient Mortgage. Elkins Park, PA: General Motors Acceptance Corporation.

Bigelow, P. Octoner 4, 1989. "Mortgage Measures Required to Encourage Affordable, Energy-Efficient Housing: Why Present Underwriting iolicies Inadvertently Handicap Affordable, Energy-Efficient Housing and What Can Be Done About It, Or, How to Conquer the Monthly Payment Handicap with PITI+E." Alliance to Save Energy Conference: Making Hotwing More Affordable Through Energy Efficiency. Palatine, IL: The Bigelow Group.

Boston Edison. January 1991. What is the Biggest Challenge You Face as a Builder Today? Marketing Your New Homes to Prospective Buyers. (Brochure), Boston, MA: Boston Edison.

Brown, E. May 1991. "Research Report on Energy Savings From Weatherization Measures." Energy Rated Homes of Arkansas. Little Rock, AK: Arkansas Department of Human Services.

Brown, G. T. 1980. "The Impact of Energy on Valuc." The Real Estate Appraiser and Analyst, November/December, 5-12.

Buchert, J. March 19, 1991. Statement of the National Association of Home Builders Before the Senate Committee on Energy and Natural Resources; Subcommittee on Energy Regulation and Conservation; on Energy Efficiency Standards and Ratings. Washington, DC: National Association of Home Builders.

Building Energy Design Tool Development Council. 1985. Evaluation Procedure for Building Energy Performance Prediction Tools: Accuracy and Home. Energy Rating Systems -- Issues and Recommendations. Washington, DC: Building Energy Design Tool Development Council.

California Statewide Collaborative Process. January 1990. Report of the Statewide Collahorative Process: An Energy Efficiency Blueprint for California.

Callaway, J. W., A. G. Thurmon, and D. L. Shankle. July 1991. Adoption, Implementation and Enforcement of Commercial Building Energy Codes in New Mexico and Arizona. Richland, WA: Pacific Northwest Laboratory. July.

Cambridge Systematics, Inc. of Berkeley, California and Regional Economic Research, Inc. of San Diego, California. Octoher 1989. Residential End-Use Energy Consumption: A Survey of Conditional Demand Estimates. Report CU-6487. Palo Alto, CA: Electric Power Research Institute.

Carlsmith, R. S., W. U. Chandler, J. E. McMahon, and D. J. Santini. January 1990). Energy Efficiency: How Far Can We Go? Oak Ridge, TN: Oak Ridge National Laboratory.

Chase, S. H., J. S. Manion, and J. Garcia-Hohman. August 1981. Documentation of the Lending Process. Bethesda, MD: Andrulis Research Corporation.

City of Fort Collins Light and Power. 1990. Energy Score, It's a Smart Move. (Brochure), Fort Collins, CO: City of Fort Collins Light and Power.

Cohen, S. and C. Goldman. 1991. "Between Two Decades: Weatherization Report Card. Home Energy, X, 3()-26. 
Cohen, S. D., C. A. Goldman, and J. P. Harris. Fehruary 1991. Measured Energy Savings and Economics of Retrofitting Existing Single-Family Homes: An Update of the BECA-B Iatabase. Berkeley, CA: Lawrence Berkeley Laboratory.

Corgel, J. B. 1982. "Measuring Energy Efficiency for Selection and Adjustment of Comparable Sales." The Appraisal Journal, January, 71-77.

Cox, M. K., A. Ackerman, L. J. Schuck, S. Heard, J. Harris, and C. Blumenstein. 1984. "User Need of a Home Energy Rating System in the Mortgage Process." Proceedings of the Summer Study in Energy Efficient Buildings: What Works: Documenting Energy Conservation in Buildings. West Newton, MA: Energyworks.

Croft, J. D. 1989. "Energy-Efficient Mortgage Program and Revised Energy Addendum." Memo to all Freddie Mac Sellers and Servicers, Reston, VA: Federal Home Loan Mortgage Corporation.

Crowe, D. 1989. "Mortgage Interest Rates and Housing Affordability." Housing Economics, May, 7-8.

Curtis, J. March 1989. Guidelines for a Uniform Energy Efficient Mortgage Program. Palo Alto, CA: Bay Area Energy Consultants.

Curtis, J. August 17, 1991. The Energy Efficient Mortgage (EEM) Concept. Palo Alto, CA: The Association of Energy Efficient Service Companies.

Davis, B. E. September 1991. The Impact of Air Distribution System Leakage on Heating Energy Consumption in Arkansas Homes. Fayettville, AR: Home Comfort.

DeLisle, J. R. 1984. "Residential Appraisal: A Behavioral Approach to Energy Efficiency." The Appraisal Journal, July, 41-47.

"Efficiency Investments Pay 3-8 Times as Much as Putting Money in the Bank." 1989. Home Energy Stars, Winter, 3.

Eley Associates. December 1990. "California Home Energy Rating System: Phase One Proposal." San Francisco, CA: Eley Associates.

Energy Crafted Home. n.d. Promoting the Construction of Energy Efficient Homes. (Brochurc).

The Energy Consultants. April 1991. "The Energy Efficient Mortgage: A Mini Summary of Measured Results." Palo Alto, CA: Bay Area Energy Consultants.

The Energy Efficient Mortgage Coalition. August 1988. "Energy Efficient Mortgage Uniform Loan Processing Proceedings Steering Committee, Working Papers." Palo Alto, CA: Bay Area Energy Consultants.

"The Energy Efficient Mortgage Program." 199(). The Energy Specialist, 6.

Energy Efficient Mortgage Uniform Loan Processing Procedures Stecring Committce. 1988. A VA, FHA, Fannie Mae and Freddie Mac Uniform Program to Allow Loan Applicants to Make Needed Energy Improvements at the Time of Purchase and/or Refinance. Energy Efficient Mortgage Coalition, Palo Alto: Bay Area Energy Consultants. 
Energy Information Administration. 1987. Residential Energy Consumption Survey 1983/84. Washington, DC: United States Department of Energy.

1987. Household Energy Consumption and Expenditures 1987, Regional. Washington, DC: United States Department of Energy.

Energy Rated Homes of America. 1990. Energy Efficient Mortgages. (Brochure), Little Rock, AR: Energy Rated Homes of America.

Energy Rated Homes of Alaska. 1990. The Smart Way to Buy or Sell a Home: For Alaskans Who Don't Have Money to Burn. (Brochure), Anchorage, AK: Energy Rated Homes of Alaska.

1990. A Consumer's Guide to a Home Energy Rating. (Brochure), Anchorage, AK: Energy Rated Homes of Alaska.

Energy Rated Homes of Arkansas. 1990. Step Up to Affordable Comfort. (Brochure), Little Rock, AR: Energy Rated Homes of Arkansas.

1990. Smart Buy. This House Rated by Energy Rated Homes of Arkansas. (Brochure), Little Rock, AR: Energy Rated Homes of Arkansas.

Energy Rated Homes of Vermont. 1988. The Energy Efficient Mortgage. (Brochure), Burlington, VT: Energy Rated Homes of Vermont.

Electric Power Research Institute. November 1990. Energy Use and Changing Lifestyles. EPRI Report CU-7069. Berkeley, CA: Lawrence Berkeley Laboratory.

"Exploring the Potential for a Uniform Energy Efficient Mortgage Program." 1990. The Energy Specialist, 6, 28-31.

Faesy, A. R. 1988. "Case Study in Success: Vermont's Energy Rated Homes." Home Energy, 5, 29-31.

n.d. Energy Efficient Mortgage Pilot Program, Program Description. Burlington, VT: Vermont Housing Finance Agency.

Faesy, A. R., P. Cillo, B. Hamilton, and B. Sachs. March 1988. The Energy Efficient Mortgage: Creative Financing for Energy Improvements in Vermont. Burlington, VT: Energy Rated Homes of Vermont.

Federal National Mortgage Assuciation. n.d. Fannie Mae's Energy Efficient Mortgage Program. Washington, DC: Federal National Mortgage Association.

. Decemher 1984. "Product Highlights: Energy Conservation Home Fin incing." Proceedings of the Solar Buildings Comference, March 1985. Silver Spring, MD: IC "Associates.

159). Energy Conservation Home Financing. (Brochure), Washington, DC: Federal National Mortgage Association. 
Federal National Mortgage Association Training Center. July 1989. Exploring the Potential for a Uniform Energy Efficient Mortgage Program. Washington, DC: Federal National Mortgage Association.

Federal Register. April 19, 1991. "Executive Order 12759 of April 17, 1991." Vol. 56, No. 76.

Florida Department of Community Affairs. 1991. "CHAS Program Helps State and Local Governments Address Florida's Affordable Housing Needs." Florida Planning, 3:1,7.

Flur, Marc. 1991. Survey of Residential Energy Efficiency Programs. Research Triangle Park, NC: North Carolina Alternative Energy Corporation.

Foute, S. J. 1982. "The Energy Mortgage Value Method." The Real Estate Appraiser and Analyst, Spring, 5-9.

Foute, S. J., F. F. Leigh, and D. E. Boyson. 1984. "Energy Mortgage Valuation: From Theory to Practice." Appraiser and Analyst, Summer, 22-27.

Friedrichs, M. n.d. Energy Efficient Mortgages. Washington, DC: Office of Planning and Analysis, U.S. Department of Energy.

Frey, D. J. n.d. Energy Efficiency Programs and Home Energy Rating Systems: Activities in Colorado. Boulder CO: Architectural Energy Corporation.

GMAC Mortgage Corporation. January 1990. Plug Into the Business Opportunity of the Future. Elkins Park, PA: General Motors Acceptance Corporation.

Gallagher, J. T. and D. J. Desmond. 1984. "Pennsylvania's Home Energy Scorecard: Development and Implementation." Proceedings of the American Council for an Energy-Efficient Economy, 1984 Summer Study on Energy Efficiency in Buildings. Washington, DC: American Council for an Energy Efficient Economy. B82-B93.

Garrett-Price, B. A., P. L. Hendrickson, and T. A. Williams. May 1982. Overview of Existing Residential Energy Efficiency Rating Systems and Measuring Tools. Richland, WA: Pacific Northwest Laboratory.

GeRue, G. ed. 1988/89. Home Energy Rating News. Little Rock, AR: Energy Rated Homes of America. Special Edition, Winter.

ed. 1989. Home Energy Rating News. Little Rock, AR: Energy Rated Homes of America. No. 2, Fall.

Gladhart, P. M., B. Morrison, and J. Zuiches. 1987. Energy and Families. Institute for Family and Child Study. East Lansing, MI: Michigan State University Press.

Goldman, C. A. May 1988. Retrofit in U.S. Multifamily Buildings: Energy' Savings, Costs, and Economics, Vol. 1. Berkeley, CA: Lawrence Berkeley Lahoratory.

Good Cents@ Division Brochure. n.d. Builder Information Guide. (Brochure), Atlanta, GA: Southern Electric International. 
n.d. Good Cents® Homes. (Brochure), Atlanta, GA: Southern Electric International.

n.d. Good Cents ${ }^{\circledR}$ Marketing Services: A Threefold Approach to Utility Marketing Services.

(Brochure), Atlanta, GA: Southern Electric International.

- n.d. Residential Load Calculations. (Brochure), Atlanta, GA: Southern Electric International.

1987. Good Cents® Residential Building Energy Progran for PC's. (Brochure), Atlanta, GA: Southern Electric International.

. 1990. Good Cents® Utilities. (Map), Atlanta, GA: Southern Electric International. November.

. 1990. Residential Duct Design. (Brochure), Atlanta, GA: Southern Electric International.

1991. Fact Sheet. (Brochure), Atlanta, GA: Southern Electric International.

. 1991. Super Good Cents/Northwest Energy Code Video Training Series. (Brochure), Atlanta, GA: Southern Energy International. March.

Harrington, B. K. and Baston, D. 1990. "One Utility's Early Experience with the Good Cents® Home Program." The Energy Specialist, 6, 10-13.

Harris, J. and C. Blumenstein. 1984. "Implementation Issues." Proceedings: What Works: Documenting Energy Conservation in Buildings. Berkeley, CA: Lawrence Berkeley Laboratory. 43-68.

Hellman, M. July 1991. Investigation into Electric Utility Incentives for Acquisition of Conservation Resources. Portland, OR: Oregon Public Utility Commission.

Henderson, Z. P. 1987. "Energy Efficient Houses Spell Profit." Human Ecology Forum, 16, 2.

Hendrickson, P. L. October 1983. Liability Aspects of Home Energy-Rating Systems. Richland, VA: Pacific Northwest Laboratory.

1986. Review of Existing Residential Energy Efficiency Certification and Rating Programs. Richland, WA: Pacific Northwest Laboratory.

. 1988. "Home Energy Rating Systems: Information to Increase Efficiency." Home Energy, 5, 22-26.

August 1988. "Implementation of Voluntary Residential Energy Efficiency Rating/Labeling Systems." Proceedings: What Works: Energy Conservation in Buildings. Richland, WA: Pacíic Northwest Laboratory.

1989. "Incorporating Energy Efficiency into Residential Appraisals." The Real Estate Appraiser and Analyst, Summer, 25-33.

Hendrickson P. L., B. M. A. Garrett-Price, and T. A. Williams. Octoher 1982. Review of Existing Energy-Efficiency Rating Systems and Measuring Tools. Richland, WA: Pacific Northwest Lahoratory. 
Hirshen, A. 1981. Energy. From the Director's Office of the Federal Home Loan Bank System. Washington, DC: Federal Home Loan Board, Office of Community Investment.

Holloway, T. M. and R. M. Rosenblatt. 1990. "The Trends and Outlooks for Foreclosures and Delinquencies." Mortgage Banking, Octoher, 45-47.

"Home Energy Rating Systems: Seeking Consensus for a National Policy." The Energy Specialist, 6, 32-34.

"How ERH Star Ratings Relate to Energy Costs." 1989. Home Energy Stars, Winter, 7.

Howard, B. and W. Prindle. July 1991. Better Building Codes for Energy Efficiency. Washington, DC: Alliance to Save Energy.

Huang, Y. J., B. Dickenson, C. Hsui, A. H. Rosenfeld, and B. S. Wagner. August 1984. "Home Energy Rating Systems: Sample Approval Methodology for Two Tools." Doing Better: Setting an Agenda for the Second Decade: Volume B, Proceedings From the Panel on New and Existing Single-Family Residences. Washington, DC: American Council for an Energy Efficient Economy. B135-B143.

- March 1985. "Home Energy Rating Systems: Sample Approval Methodology for Three Tools." Doing Better: Setting an Agenda for the Second Decade. Berkeley, CA: Lawrence Berkeley Laboratory.

Hubert, J. and J. Luboff. August 1982. "The Washington Program: A Shelter Industry Residential Energy Evaluation Program." Proceedings: What Works: Energy Conservation in Buildings. Santa Cruz, CA: Energy Conservation in Buildings Symposium. 532-533.

"HUD Actions Encourage Home Energy Efficiency." 1989. Home Energy Stars, 2, 3.

Hughes, R. 1989. "Congress Studies Energy Ratings." Home Energy Stars, Summer, 3.

1990. ed. Home Energy Rating News. Little Rock, AR: Energy Rated Homes of America. Vol. 2, Summer.

1990. "Home Energy Rating Systems: Seeking Consensus for a National Policy." Home Energy Rating News, 2, 1.

January 7, 1991. Home Energy Rating Systems and Energy Efficient Mortgages Who's Who. Little Rock, AR: Energy Rated Homes of America.

Idaho National Engineering Laboratory, Los Alamos National Laboratory, Oak Ridge National Laboratory, Sandia National Laboratories, and Solar Energy Research Institute. 1990. The Potential of Renewable Energy - An Inter-laboratory White Paper. Golden, CO: National Renewable Energy Laboratory.

Ideal Energy Home. November 15, 1991. "Ideal Energy Home Guide." (Advertisement), The Denver Post, Classified advertising supplement. November 15. 
Joint Center for Housing Studies of the Massachusetts Institute of Technology and Harvard University. May 1986. The Elfect of the Shelter Industry Energy Rated Homes ${ }^{T M}$ System on Homeownership. Boston, MA: Joint Center for Housing Studies of the MIT and Harvard University.

Johnson, A. September 199(). HUI) Credits Energy Efficient Homes. Cleveland, OH: Edgell Communications.

Septemher 1990. Insulation: Outlooks for the New Home Market. Cleveland, OH: Edgell Communications.

Judkoff, R. n.d. A Quantitative Procedure for Evaluating Home Energy Rating System (HERS) Calculation Methods. Golden, CO: Solar Energy Research Institute.

. October 1988. "Validation of Building Energy Analysis Simulation Programs at the Solar Energy Research Institute. Energy and Buildings. Lausanne, Switzerland: Elsevier Sequoia S.A.

Kailo, N. 1991. "Channel Buyers Into an Energy-Saving Alternative." Real Estate Today, May, 40-41.

Kempton, W. and M. Neiman, eds. 1987. Energy Efficiency, Perspectives on Individual Behavior. Washington, DC: American Council for an Energy Efficient Economy.

Klareich, F., J. R. Hochanadel, and D. Devoe. November 1990. A Final Report: A Guide to Implementing a Home Energy Rating System. Washington, DC: CRS Incorporated.

Koomey, J. G., C. Atkindon, A. Meier, J. E. McMahon, S. Boghosian, B. Atkison, I. Turiel, M. P. Levin, B. Nordam, and P. Chan. July 1991. The Potential for Electricity Efficiency Improvements in the. U.S. Residential Sector. Berkeley, CA: Lawrence Berkeley Laboratory.

Lashoff, D. A. and D. A. Tirpak, eds. December 1990. Policy Options for Stabilizing Global Climate. Washington, DC: United States Environmental Protection Agency.

Lehman, H. J. Octoher 14, 1989. "Energy Efficient' Mortgages Can Increase Buying Power." The Washington Post. E3.

Lessne, G. J. Novemher 1987. Evaluation of Energy Conservation Assistance Programs. Providence, RI: University of Rhode Island.

Levine, M. and S. Maves. 1982. "A Description and Assessment of Energy Labels for Houses." Proceedings of the Summer Study in Energy Efficient Buildings: What Works: Documenting Energy Conservation in Buildings. West Newton, MA: Energyworks.

Levy, D. E. 1987. "Adjusting for Energy Efficiency in Residential Appraisal." The Appraisal Joumal, July, 457-462.

Lodge, M. April 1991. "Energy Efficient Mortgages at Last." l.(0)1 Home Ideas. Palo Alto, CA: The Association of Energy Efficient Mortgage Service Companics.

Massachusetts Auduhon Socicty. Decemher 1981. Envirommental Science Department Booklet. Lincoln, MA: Massachusetts Auduhon Society. 
Massachusetts Home Energy Rating. n.d. The Massachusetts Home Energy Rating System Project. (Brochure). Boston, MA: Mass-Save.

McCracken, L. 1989. "Appraiser Recommends Local Benchmark." Home Energy Stars, Summer, 7.

Miller, R. M. 1986. "The Energy Efficient Mortgage: New Lending Policies to Boost Bank Acceptance." Proceedings From the American Council for an Energy-Efficient Economy 1986 Summer Study on Energy Efficiency in Buildings. Washington, DC: American Council for an Energy Efficient Economy. 4.108-4.118.

Miller, R. M., C. B. Smith, T. Davis, and P. W. Turnball. 1985. "Financing Energy Conservation Through Home Mortgages: Implications for Utility Planners." Proceedings of the Second Great $P G$ and E Energy Expo, 1985. Elmsford, NY: Pergamon Books Inc. 349-354.

Mills, E. February 1987. "Higher Mortgages, Lower Energy Bills: The Real Economics of Buying an Energy-Efficient Home." Fifth Annual International Energy Efficient Building Conference and Exposition. Berkeley, CA: Lawrence Berkeley Laboratory.

National Analysts, SRC, and QEI, Inc. March 1989. Residential Customer Preference and Behavior: Market Segmentation Using CLASSIFY (TM). Electric Power Research Institute, Report EM-5908.

National Association of Home Builders. 1983. NAHB Thermal Performance Guidelines. Washington, DC: National Association of Home Builders.

National Collaborative. March 1992. Going National with HERS and EEMs: Issues and Impacts, The Collected Papers of the National Collaborative. (NREL/TP-261-4706) Golden, CO: National Renewable Energy Laboratory.

National Energy Program Working Group Standing Committee on Energy, National Association of Home Builders. May 10, 1990. National Association of Home Builders Energy Program. Washington, DC: National Association of Home Builders.

National Institute of Building Sciences. April 15, 1985. Final Report of the Project Committee: Voluntary Energy Rating Systems for Residences. Washington, DC: National Institute of Building Sciences.

National Renewable Energy Laboratory. 1990. BuilderGuide. Washington, DC: Passive Solar Industries Council.

Natural Resources Deîense Council. 1991. Environmental Benefits. Washington, DC: Natural Resources Defense Council.

Nebraska Energy Office. n.d. Dollar and Energy Saving Loans at 5\% Interest for Home, Building, and System Improvements. (Brochure), Lincoln, NE: Nebraska Energy Office.

n.d. Dollar and Energy Saving Loans at 5\% Interest: Refrigerators, Freesers, Dishwashers, Clothes Washers. (Brochure), Lincoln, NE: Nebraska Energy Office.

Newbraugh, F. 1990. "Home Energy Rating Systems: The Problem." The Energy Specialist, 6, 35-36. 
Nichols, A. K. and D. B. Belzer. 1988. Estimation of Building Sector Energy Savings Potential from OBCS Funded Research: A Technical Support Document. Richland, WA: Pacific Northwest Laboratory.

Nichols, J. 1988. "M.P.G. For Houses: The Uphill Battle of Energy Rated Homes." Spectrum, July, 6-19.

North Carolina Exemplary Home Program. August 1990. Draft Implementation Plan.

Nybo, J. H. March 1990. The Energy-Efficient Mortgage: A Lender-Appraiser Manual. Boise, ID: The Idaho Energy Efficient Mortgage Program.

Office of Building Technologies. December 1991. Energy Efficient Mortgages. (Factsheet), Washington, DC: U.S. Department of Energy.

Olin, H. B. 1985. "Annual Energy Technology Conference and Exposition." Energy Technology, 12, 666-678.

Passive Solar Industries Council. 1989. Passive Solar Design Strategies: Guidelines for Builders. Washington, DC: Passive Solar Industries Council.

Passive Solar Industries Council. 1991. Passive Solar Design Strategies: Remodeling Guidelines for Conserving Energy at Home. Washington, DC: Passive Solar Industries Council.

Patterson, P. ed. 1990. Home Energy Rating News. Little Rock, AR: Energy Rated Homes of America. Vol. 2, Spring.

Pendleton, S. 1991. "Refinancing of Home Loans Is Near a Peak." The Christian Science Monitor, December, 6.

Perlin, G. L. 1990. Managing Your Mortgage Pipeline: A Hedging Guide. Washington, DC: Federal National Mortgage Association.

Pratt, R. G., C. C. Conner, M. K. Drost, N. E. Miller, B. A. Cooke, M. A. Halverson, B. A. Lebaron, R. G. Lucas, J. Jo, E. E. Richman, K. G. Ritland, W. F. Sandusky, M. E. Taylor, and S. G. Hauser. December 1990. Significant ELCAP Analysis Results: Summary Report. Richland, WA: Pacific Northwest Laboratory.

Prindle, W. R. 1988. "Testing the Energy-Efficient Mortgage Program in Connecticut." Home Energy, 5, 27-29.

W. R. March 27, 1990. "Testimony Before The Subcommittee on Energy Regulation and Conservation; Committee on Energy and Natural Resources; United States Senate." Washington, DC: Alliance to Save Energy.

Prindle, W. R and M. W. Reid. 1990. "Making Housing More Affordable Through Energy Efficiency." The Energy Specialist, 6, 23-27.

Ragas, W. R. and J. R. Wyatt II. 1978. "Residential Energy Capitalization Technique and Single-Family Home Underwriting." The Real Estate Appraiser and Analyst, January/February, 13-18. 
Rakow, L. L. 1989. "Measuring Home Energy Efficiency." The Real Estate Appraiser and Analyst. Summer, 34-36.

"Rating Systems in the U.S.A.: How Many Are There?" 1590. Home Energy Rating News, 2, 1-7.

RISE. n.d. The Sign of an Energy Efficient Home. (Brochur ?), Providence, RI: RISE.

Ritschard, R., E. Vine., B. K. Barnes, C. Husui, I. Reyes, E. J. Cairns, and M. Levine. March 1988. "California Home Energy Rating and Labelling Demonstration Program: What Did We Learn?" Energy Analysis Program, FY 1987: Applied Science Division Anmual Report. Berkeley, CA: Lawrence Berkeley Laboratory.

Rogers, B. 1991. "Manageable Energy Management." Public Power, 49, 20-25.

Roll, J. B., W. R. Haynie, and T. Davis. May 199.4. "Home Energy Rating System." Proceedings of the Conference on Utility Conservation Programs: Planning, Analysis and Implementation. Boston, MA: Peter Merrill Associates, Inc. 17.1-17.18.

Rosenfeld, A. H., C. Atkinson, J. Koomey, A. Meier, and R. Mowris. February 1991. "Policy Implications of Greenhouse Warming: Compilation of Supply Curves for U.S. Buildings." Residential and Commercial Energy Management. Berkeley, CA: Lawrence Berkeley Laboratory.

Rosenfeld, A. H. and B. S. Wagner. 1982.. "Technical Issues for Building Energy Ratings." ACEEE Conference. Santa Cruz, CA: American Council for an Energy Efficient Economy. 338-402.

Rudeen, S. K. and N. Hanna. December 1990. "The Energy Efficient Mortgage and Affordahility in Washington State." Washington State Energy Office.

Schuck, L., J. Millhone, J. Harris, and C. Blumenstein. August 1984. "Defining Energy Efficiency in Residential Lending Practices." Proceedings of the Summer Study in Energy Efficient Buildings: What Works: Documenting Energy Conservation in Buildings. Washington, DC: Alliance to Save Energy.

Seiter, D. L. 1988. Austin Energy Star: A Home Energy Rating Status Report. Austin, TX: City of Austin Resource Management Department.

Simon, V. 1989. "Energy Efficient Mortgages are Gaining Appeal." Real Estate Finance Today, 6:1, 12.

Socolow, R. 1978. "Variation Across Houses in Energy Consumption for Heating." Saving Energy in the Home. Princeton, N.J.: Princeton University Press.

Solar Buildings Conference Proceedings. July 1985. "Product Highlights: Energy Conservation Home Financing. Solar Buildings: Realities for Today-Trends for Tomorrow: Silver Spring, MD: MCC Associates, Inc. 281-282.

Sondregger, R. C. 1978. "Movers and Stayers - The Resident's Contrihution of Variation Across Houses in Energy Consumption for Heating." Saving Entergy in the Home. Princeton, NJ: Princeton University Press. 
Solar Energy Research Institute. 1989. "Predicting Home Energy Use With STEM." Science \& Technology in Review, 11, 7.

Spaid, E. L. 1991. "Some Mortgages Cut Heating Bills." The Christian Science Monitor, Decemher, 6.

Steel, G. B., J. Hayes, and D. A. Andrejko. 1983. "The California Point System-Energy and Rating Systems Model for States." Eighth National Solar Energy System.s: Progress in Passive Solar Energy Desigri. New York: American Solar Energy Society. 567-572.

Swartz, D. K. 1991. "Energy Score: Home Energy Rating Experience in Fort Collins, Colorado." American Public Power Association Energy/Customer Services \& Communications Workshop. Colorado Springs, CO. November 4.

Swisher, J. N., J. M. Gregerson, M. J. Holtz, D. A. Andrejko, and J. Hayes. 1987. "A New Computerized Home Energy Rating System for Fort Collins, CO." Proceedings of the 12th National Passive Solar Conference. Boulder, CO: American Solar Energy Society. July 12. 360-364.

Tarini, E., J. Harris, and C. Blumenstein. 1984. "Methodology for Adding a Home Energy Rating System to an Existing RCS Audit Program." Report of Summer Study in Energy Efficient Buildings: What Works: Documenting Energy Conservation In Buildings. Watertown, MA: Energyworks.

Teutsch, J. M. and J. R. Faulstich. 1985. "The Energy Factor in the Market Equation." Mortgage Banking, May, 75-80.

Uniform Energy Efficient Mortgage Steering Committee. 1989. Guidelines for an Energy Efficient Mortgage Program. Palo Alto, CA.

U.S. Department of Energy. May 1989. Energy Conservation Standards for New Residential Buildings. Washington, DC: DOE.

1991/1992. National Energy Strategy: Powerful Ideas for America. Washington, DC: DOE.

U.S. Department of Energy, Office of Technical and Financial Assistance. 1990. A Guide to Implementing a Home Energy Rating System. Washington, DC: CSR Incorporated.

U.S. Department of Housing and Urban Development. 1989. 1989-1990 Programs of HUD. Washington, DC: HUD.

U.S. Department of Housing and Urban Development, Office of Policy Developmeni and Research, Research Utilization Division. June 1990. 1989 Report to Congress on the Federal Home Loan Mortgage Corporation. Washington, DC: HUD.

June 1990. 1989 Report to Congress on the Federal No ional Mortgage Associationl. Washington, DC: HUD.

Veterans Administration. December 10, 1985. Leans to Purchase Homes and Make Energy Comservation Improvements. Washington, DC: Department of Veterans, Benefits. 
Vine, E., B. K. Barnes, and R. Ritschard. 1987. Energy Conservation Program Evaluation: Practical Methods, Useful Results: Proceedings. Berkeley, CA: Lawrence Berkeley Laboratory.

September 1987. "Evaluation of the Implementation of Home Energy Rating Systems." Energy Conservation Program Evaluation: Practical Methods and Useful Results: Proceedings Volume 2. August 20 and 21 Sessions, Chicago, IL. Berkeley, CA: Lawrence Berkeley Laboratory.

February 1987. Home Energy Rating Systems: Program Descriptions. Berkeley, CA: Lawrence Berkeley Laboratory.

February 1987. Implementation of Home Energy Rating Systems. Berkeley CA: Lawrence Berkeley Lahoratory.

. 1988. "Implementing Home Energy Rating Systems." Energy, 13, 401-411.

. March 1988. "Home Energy Rating Systems: An Analysis of a Voluntary Demand-Side Management Program." Third National Conference on Utility Demand-Side Management Programs: ISSM Strategies in Transition. Berkeley, CA: Lawrence Berkeley Laboratory.

Vine, E., B. K. Barnes, R. Ritschard, E. J. Cairns. March 1988. "Evaluation of the Implementation of Home Energy Rating Systems." Energy Analysis Program, FY 1987: Applied Science Division Anmual Report. Berkeley, CA: Lawrence Berkeley Laboratory. 3.6-3.7.

Vine, E., B. K. Barnes, R. Ritschard, E. J. Cairns, and M. Levine. March 1988. "Evaluation of the Implementation of Home Energy Rating Systems." Energy Conservation Program Evaluation: Practical Methods and Useful Results: Proceedings Volume 2, August 20 and 21 Sessions. Berkeley, CA: Lawrence Berkeley Laboratory.

Vine, E., B. K. Barnes, R. Ritschard, S. Limaye. October 1987. "Home Energy Rating Systems: An Analysis of a Voluntary Demand-Side Management Program." Third National Conference on Utility Demand-Side Management Programs: DSM Strategies in Transition. Berkeley, CA: Lawrence Berkeley Laboratory. 30.1-30.10, Section 30.

Vorics, R. 1984. Comprehensive Implementation and Promotion Plan for the Austin Energy Star Program. Denver, CO: Infinite Energy.

September 1985. Austin Energy Star Builders Handbook. Austin, TX: Resource Management Department.

1985. "Marketing the Austin Encrgy Star Program." Energy Services Exchange On-Site Workshop: Developing an Energy Rating System. Austin, TX: Conference sponsored hy the American Public Power Administration and the Resource Management Department.

. February 1985. Market Penetration Projections, Austin Energy Star Prosmm. Denver, CO: Infinite Energy.

Fehruary 1985. Market Study and Evaluation Design, Austin Energy Star Program. Denver, $\mathrm{CO}$ : Infinite Energy. 
August 1986. "Marketing the Austin Energy Star Program: Using Private Sector Techniques to Market a Public Sector Program." Proceedings from the ACEEE 1980 Summer Study on Energy Efficiency in Buildings, Volume 5, Marketing. Washington, DC: American Council on an Energy Efficient Economy.

. January 1987. Phase I, Briefing Paper Number One: Factors to be Considered in Developing a Building Energy Efficiency Rating System for Existing Homes in Montgomery County Including Summary of Case Studies. Washington, DC: W.S. Fleming \& Associates for Department of Environmental Protection.

1988. "Evaluation of Seven Home Energy Rating Programs for Existing Single-Family Homes." Utility and Private Sector Conservation Programs Conference, Pacific Grove, CA. Washington, DC: American Council for an Energy Efficient Economy.

January 1988. Final Report: Task 34-1, Ft. Collins Light and Power Home Energy Rating Program, Focus Group Results. Golden CO: NEOS Corporation.

1988. A Guide for Energy \$aver Homeowners: Operating and Equipping Your Home for Maximum Comfort and Savings. Denver, CO: Infinite Energy.

. January 28, 1988. "Use of Utility Bills in the Homebuying Process." Presentation at the Princeton Center for Energy and Environmental Studies on Innovations in Utility Billing for Conservation and Customer Service. Princeton, NJ.

1988/89. "Marketing: The Key to Successful Rating Programs." Home Energy Rating News, Winter, 6-7.

1989. "Focus Group Results in Home Energy Rating System for City of Fort Collins Light and Power Department." Proceedings of Solar '89, the 14th National Passive Solar Conference. Denver, CO. New York: American Solar Energy Society.

. 1989. Recommendations for Energy Rated Homes of Vermont Marketing Strategy. Denver,

CO: Infinite Energy.

. 1989. "What Makes Rating Systems Tick?" Home Energy, 6, 22-26.

1990. "Overview of Home Energy Rating Systems." Proceedings of the 8th Annual International Energy Efficient Builders Conference and Exposition: Excellence in Housing 1990. Denver, CO: Energy Efficient Builders Association.

1991. "Making 'HERS' a Household Word." Home Energy, 8, 30-35.

January 1991. Program Redesign: B.C. Hydro Quality Plus New Home Energy Cerificition Program. Denver, CO: Infinite Energy.

Vories, R. and K. George. 1991. Analysis of Home Energy Rating Systems: Results of Brief Interviews. Denver, CO: Infinite Energy.

March 26, 1991. Summary of Preliminary Report: Survey of Home Energy Rating Systems. Richland, WA: Pacific Northwest Laboratory. 
Vories, R. and H. Misuriollo. August 1988. "Evaluation of Seven Home Energy Rating Programs fir Existing Single-Family Homes." Utility and Private Sector Comsenation Program.s, Vol. o of the 1988 ACEEE Stummer Study on Energy Efficiency in Buildings. Washington, DC: American Council on an Energy Efficient Economy.

Vorics, R. and M. Samuclson. 1984. Comprehensive Implementation and Promotion Plan for the Austin Energy Star Program. Denver, CO: Infinite Energy.

June 1988. Marketing and Promotion Strategy for Ft. Collins Energy Score Program. Denver, CO: Infinite Energy.

April 22, 1991. Quality Plus Program Marketing Recommendations. Denver, CO: Infinite Energy.

Vories, R., D. Swartz, and D. Ruc. June 1989. Focus Group Results Home Energy Rating System of Fort Collins Light and Power Department. New York: American Solar Energy Socicty.

Wadleigh, D. 1990. "Energy Efficient Mortgages for New Homes." The Energy Specialist, 6, $20-22$.

Walker, K. ed. 1990/91. Home Energy Rating News. Little Rock, AR: Encrgy Rated Homes of America. Vol. 3, Winter.

Waller, N.G. 1989. "Residential Mortgage Default: An Empirical Note on Changes in Property Value and Debi Over Time. Housing Finance Review, 8, 155-164.

. 1988. "Residential Mortgage Default: A Clarifying Analysis." Housing Finance Review, 7 , $321-333$.

Wehb, J. R. 1980. "The Influence of Solar Energy Systems in the Value of Dwellings: Theory and Practice." The Real Estate Appraiser and Analyst, January/February, 4-6.

Wilson, K. and P. Conlan. May 8, 1980. Wisconsin Home Energy Efficiency Rating System: Final Report. Atlanta GA: A\&C Enercom.

Wilson, N. W., W. G. Colborne, T. Hoffman, E. Fretz, and N. Niec. 1982. "Occupant Effects on Residential Energy Consumption." ENERGEX Conference. Ontario, Canada: University of Windsor. 57-60.

Woehrle, L. A. 1984. "Banking on Home Energy Efficiency." Public Power, 42, 40-41 and 46-50. 

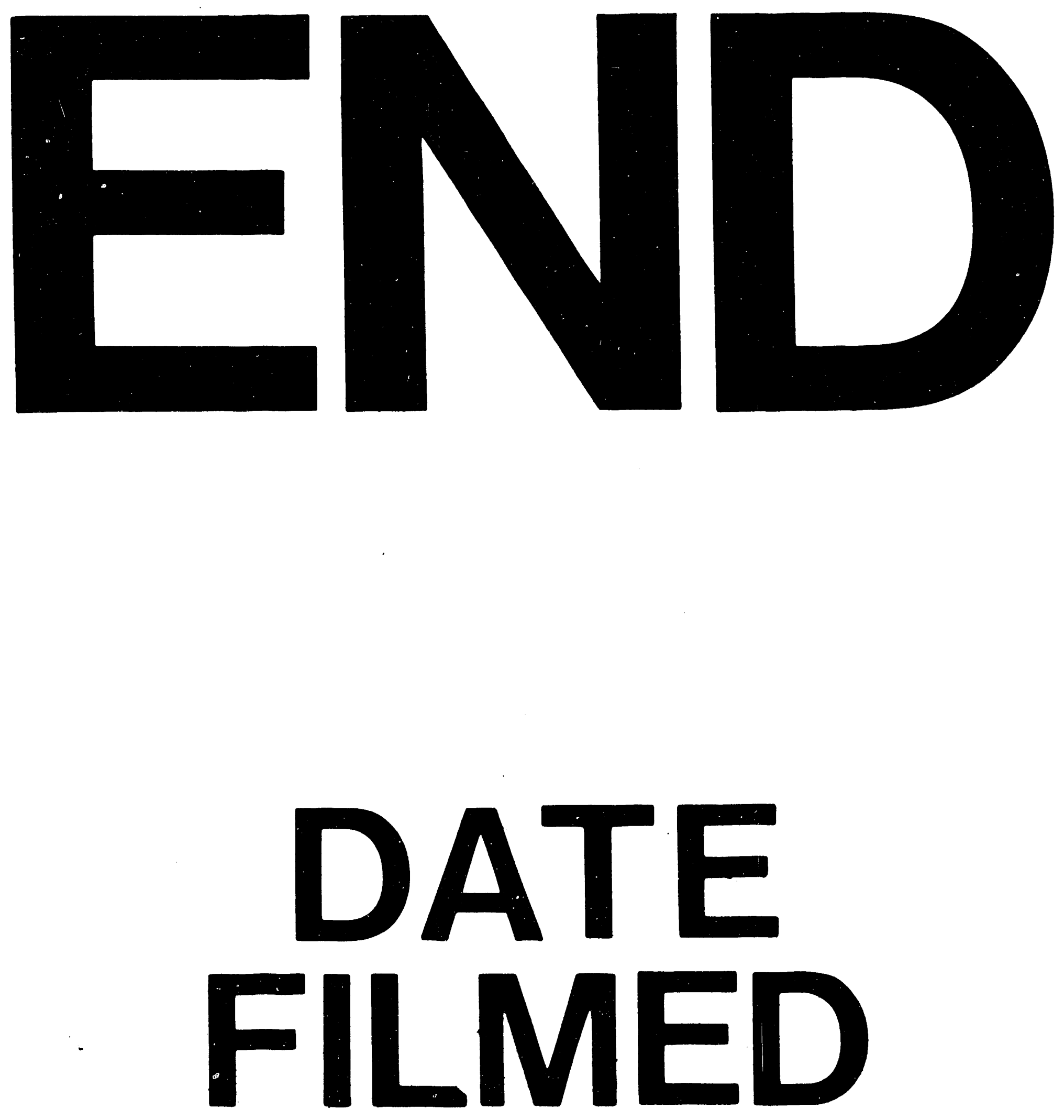

I

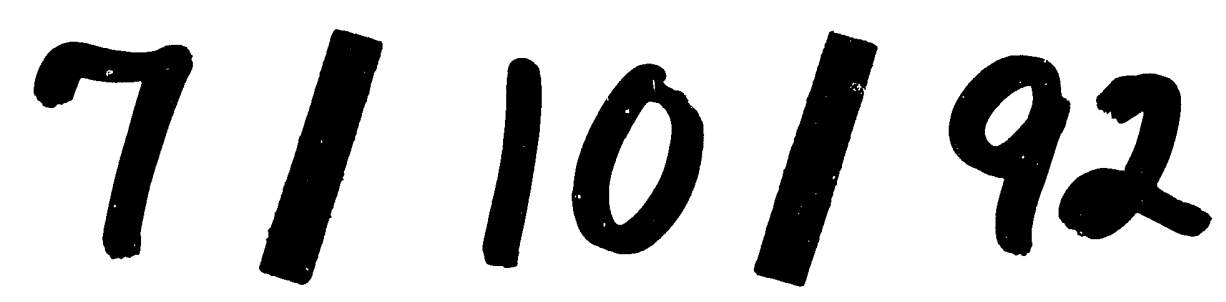


